\title{
Calibration of an Innovative Rate Sensing / Momentum Management Instrument for De-Tuned Operation and Temperature Effects
}

by

\author{
JEFFREY M. HALL, B.Eng. \\ A thesis submitted to \\ the Faculty of Graduate Studies and Research \\ in partial fulfillment of \\ the requirements for the degree of \\ Master of Applied Science \\ Ottawa-Carleton Institute for \\ Mechanical and Aerospace Engineering \\ Department of \\ Mechanical and Aerospace Engineering \\ Carleton University \\ Ottawa, Ontario \\ May $30^{\text {th }}, 2008$ \\ (C) Copyright \\ 2008, Jeffrey M. Hall
}




$\begin{array}{ll}\begin{array}{l}\text { Library and } \\ \text { Archives Canada }\end{array} & \begin{array}{l}\text { Bibliothèque et } \\ \text { Archives Canada }\end{array} \\ \begin{array}{l}\text { Published Heritage } \\ \text { Branch }\end{array} & \begin{array}{l}\text { Direction du } \\ \text { Patrimoine de l'édition }\end{array} \\ \begin{array}{l}\text { 395 Wellington Street } \\ \text { Ottawa ON K1A 0N4 } \\ \text { Canada }\end{array} & \begin{array}{l}\text { 395, rue Wellington } \\ \text { Ottawa ON K1A 0N4 } \\ \text { Canada }\end{array}\end{array}$

Your file Votre référence ISBN: 978-0-494-44041-4 Our file Notre référence ISBN: 978-0-494-44041-4

NOTICE:

The author has granted a nonexclusive license allowing Library and Archives Canada to reproduce, publish, archive, preserve, conserve, communicate to the public by telecommunication or on the Internet, loan, distribute and sell theses worldwide, for commercial or noncommercial purposes, in microform, paper, electronic and/or any other formats.

The author retains copyright ownership and moral rights in this thesis. Neither the thesis nor substantial extracts from it may be printed or otherwise reproduced without the author's permission.
AVIS:

L'auteur a accordé une licence non exclusive permettant à la Bibliothèque et Archives Canada de reproduire, publier, archiver, sauvegarder, conserver, transmettre au public par télécommunication ou par l'Internet, prêter, distribuer et vendre des thèses partout dans le monde, à des fins commerciales ou autres, sur support microforme, papier, électronique et/ou autres formats.

L'auteur conserve la propriété du droit d'auteur et des droits moraux qui protège cette thèse. $\mathrm{Ni}$ la thèse ni des extraits substantiels de celle-ci ne doivent être imprimés ou autrement reproduits sans son autorisation.
In compliance with the Canadian Privacy Act some supporting forms may have been removed from this thesis.

While these forms may be included in the document page count, their removal does not represent any loss of content from the thesis.
Conformément à la loi canadienne sur la protection de la vie privée, quelques formulaires secondaires ont été enlevés de cette thèse.

Bien que ces formulaires aient inclus dans la pagination, il n'y aura aucun contenu manquant.

\section{Canada}




\section{Abstract}

The GyroWheel ${ }^{\mathrm{TM}}$ is an innovative rate sensing and momentum management instrument. Its design is based on the structure of a dynamically tuned gyro, with the exceptions of a significantly larger rotor and tilt angle sensing, so that the device functions as a precision two axis rate sensing gyroscope. Even though the device is about the same size and power as a standard single axis momentum wheel of the same momentum class, it can also provide the same three axis momentum steering capability as a double-gimbaled control moment gyro. Therefore the GyroWheel TM allows for significant mass and cost savings of multiple wheels while still providing similar functionality, which is innovative especially in the field of small satellite attitude determination and control systems (ADCS).

Further mass and cost savings could be achieved through calibration of the system to compensatc for changes in the magnetic field strength (B field) due to temperature, making it so that the GyroWheel ${ }^{\mathrm{TM}}$ system is not required to be part of a satellite's active thermal control system. The GyroWheel ${ }^{\mathrm{TM}}$ would also provide greater functionality through the calibration of the 2 -axis rate sensing for de-tuned conditions involving changes in both the wheel speed and rotor plane tilt from the null position.

This thesis achieves greater accuracy in the performance of the GyroWheel ${ }^{\mathrm{TM}}$ system through calibration for changes in temperature, wheel speed and rotor plane tilt as well as adding additional ADCS functionality for de-tuned, non-temperature controlled operation. 


\section{Acknowledgments}

As I sit here in five metre waves in the middle of the pacific ocean, typing a portion of my thesis, laptop on my lap, swaying back and forth with my chair bungee corded to my bunk in Her Majesty's Canadian Ship ALGONQUIN, a Canadian naval warship, I think how apropos it is that I am discussing inertial navigation. More specifically, how interesting it is that I am working with satellite inertial navigation, as the only means of communication the ship has with the outside world right now is via satellite. Even more specific than that, as I grow more queasy, I think about how nice it would be to have a gyro-stabilized platform to work on...

Well, this is finally it. The time when I can sit back and think about how I am going to attempt to thank the numerous people who helped me in achieving my goals, knowing full well that the words I will write on this page will pale in comparison to my thanks and appreciation for the help that they have given me.

First off I would like to thank Doug Staley, my thesis supervisor, for taking on an electrical engineering student with an interest in broadening his knowledge in all fields of aerospace engineering, and for allowing me to do just that. I have directly applied almost everything I have learned at Carleton University in my employment within the Canadian Forces and have even passed on a small portion of your knowledge to others. Thanks for sticking with me and for your patience with my 'longish' timeline for completion.

To Fred Schultz..... owe you more than I can explain in words. I mean whoever would have thought they could say that they have actually worked on a satellite component that is now up in space. That is amazing!! Fred, you are a true gentleman scholar. I sincerely thank you for letting me have this short ride on your coattails. 
I would like to thank all the people at Bristol (Dave McCabe, Mark Senez, Jean de Carufel, and Brian Ungar), as well as the people at the inertial navigation laboratory of Defence Research and Development Centre Ottawa at Shirley's Bay (Mike Vinnins, Llyod Gallop, and Slobodan Jovic) for putting up with me popping in and out and asking all sorts of questions. Thank you for making the time.

To my parents who always encouraged me, no matter how crazy my ideas were, and to my family and my friends, thank you for being there when I needed support, or a good kick in the behind to get back on track. My friends' well placed sarcasm has always helped me to regain focus.

And finally to my beautiful, smart and patient wife Joanna, thank you for sticking with me through the long nights and weekend shifts collecting the data. Thank you for working with me through the nights, pushing each other to get done, and helping me believe that we could get through this. We finally did it!! I love you. 


\section{Contents}

Acceptance Sheet $\quad$ ii

Abstract $\quad$ iii

Acknowledgments $\quad$ iv

Table of Contents vi vi

List of Tables $\quad$ X

List of Figures $\quad$ xi

Nomenclature xiii

1 Introduction $\quad \mathbf{1}$

1.1 Thesis Overview . . . . . . . . . . . . . . . . . 1

1.2 Motivation For Testing . . . . . . . . . . . . . . . . . 2

1.3 Thesis Outline . . . . . . . . . . . . . . . 3

2 Overview of Spacecraft Systems 5

2.1 Satellite Thermal Control Systems and Heat Transfer . . . . . . . . . 6

2.1.1 Principles of Heat Transfer . . . . . . . . . . . . . . . . . . 7

2.1.2 Thermal Time Constant . . . . . . . . . . . . . . 10

2.2 Magnetic Field Changes with Temperature . . . . . . . . . . . . . 16

2.2.1 Permanent Magnet Field Strength . . . . . . . . . . . . . 18

2.2.2 Magnetic Coils ... . . . . . . . . . . . . . . . 21 
2.3 Gyroscope Theory . . . . . . . . . . . . . . . . . . 23

2.3.1 Conventional Gyroscopes . . . . . . . . . . . . . . . . . 24

2.3.2 Dynamically Tuned Gyroscopes . . . . . . . . . . . . . . 26

2.4 Momentum Management . . . . . . . . . . . . . . . . . . . . . . . . 31

3 The GyroWheel ${ }^{\text {TM }}$ System $\quad 36$

3.1 System Components . . . . . . . . . . . . . . . . . 37

3.1 .1 Spin Motor . . . . . . . . . . . . . . . 37

3.1 .2 Bearing Cartridge Subassembly . . . . . . . . . . . . 37

3.1 .3 Gimbal Assembly . . . . . . . . . . . . . . . . . . . 40

3.1 .4 Rotor . . . . . . . . . . . . . . . 43

3.1 .5 Tilt Sensors . . . . . . . . . . . . . . . . 43

3.1 .6 Torque Coils . . . . . . . . . . . . . . . . . . . . . 46

3.1.7 Drive and Control Electronics . . . . . . . . . . . . . . 47

3.2 Principle of Operation $\ldots \ldots \ldots \ldots \ldots$

3.2.1 Steerable Momentum Wheel . . . . . . . . . . . . . . . . 48

3.2 .2 External Rate Sensing . . . . . . . . . . . . . . . . . . . 49

3.2 .3 Performance Characteristics . . . . . . . . . . . . . . . 49

3.2 .4 Measured Values . . . . . . . . . . . . . . . . 50

4 Dynamically Tuned Gyroscope Dynamics, Rate Sensing, and Testing Theory 53

4.1 Dynamics of Dynamically Tuned Gyroscopes . . . . . . . . . . . 53

4.1 .1 Tuning Condition of the DTG . . . . . . . . . . . 54

4.1 .2 DTG Control System Design . . . . . . . . . . . . . 58

4.1 .3 Gyroscope Error Terms . . . . . . . . . . . . . . . 60

4.1.4 Errors Specific to the DTG . . . . . . . . . . . 63

4.1 .5 DTG Rate Sensing . . . . . . . . . . . . . . . . . . . 69

4.2 Testing and Calibration of Dynamically Tuned Gyroscopes . . . . . 72 4.2 .1 Multi-Position Testing . . . . . . . . . . . . . . . 73

4.3 Dynamics of the GyroWheel ${ }^{\mathrm{TM}}$ System . . . . . . . . . 75 4.3.1 GyroWheel ${ }^{\mathrm{TM}}$ Frames of Reference Definitions . . . . . . 75 
4.3.2 GyroWheel ${ }^{\mathrm{TM}}$ Lagrangian Dynamics Formulation . . . . . . . 75

4.3 .3 Actuator Model . . . . . . . . . . . . . . . . . . . . . . 83

4.3.4 Linearizing About an Operating Point . . . . . . . . . . 85

4.4 GyroWheel ${ }^{\mathrm{TM}}$ Rate Sensing . . . . . . . . . . . . . . . . 88

4.4.1 GyroWheel ${ }^{\mathrm{TM}}$ Flight Rate Sensing Equation . . . . . . . 88

4.4 .2 GyroWheel ${ }^{\mathrm{TM}}$ Error Terms . . . . . . . . . . . . . . . 89

4.4.3 GyroWheel ${ }^{\mathrm{TM}}$ Ground Calibration ............. 91

5 GyroWheel ${ }^{\text {TM }}$ Testing $\quad 94$

5.1 Equipment Utilized . . . . . . . . . . . . . . . . . . . . . . . 94

5.1 .1 Contraves Motion Table . . . . . . . . . . . . . . . 95

5.1.2 Temperature Control . . . . . . . . . . . . . . 96

5.1 .3 Vacuum Chamber . . . . . . . . . . . . . . . 97

5.1.4 Data Monitoring and Collection . . . . . . . . . . . . 97

5.2 Description of Tests . . . . . . . . . . . . . . . . . . . . . . . 101

5.2.1 Turn On - Turn On Repeatability . . . . . . . . . . . . . . . . 101

5.2 .2 Drift Test . . . . . . . . . . . . . . . . 101

5.2 .3 Multi-Position Testing ............... 103

5.2.4 Temperatures During Testing . . . . . . . . . . . . . 105

5.2.5 Tilt Sweep Testing . . . . . . . . . . . . . . . 107

5.2.6 Wheel Speed During Testing . . . . . . . . . . . . 107

5.2.7 Combined Temperature and Tilt Sweep Testing . . . . . . . . 107

5.3 Data Extraction and Clean-up . . . . . . . . . . . 108

6 Analysis and Conclusions 110

6.1 Singular Value Decomposition . . . . . . . . . . . . . . . 110

6.2 Temperature Calibration . . . . . . . . . . . . . . . . . . 114

6.3 In-Flight Rate Sensing Temperature Calibrated Terms . . . . . . . . 118

6.4 Final Calibration Coefficients . . . . . . . . . . . . . . . . 120

6.4.1 Discussion of Final Results . . . . . . . . . . . . . . . . . . 124

6.5 Summary of Contributions . . . . . . . . . . . . . . 125

6.5.1 Future Work . . . . . . . . . . . . . . . . . . . 129 
A Calibration Coefficients for Various Multi-Position Analyses used to Determine Negligible Coefficient Terms

B Set of Current Plots Versus Temperature for Combined Temperature and Tilt Sweep Testing

C Calculated Calibration Coefficients at Null Tilt Conditions 


\section{List of Tables}

2.1 Estimated Parameters for GyroWheel ${ }^{\mathrm{TM}}$ Thermal Time Constant . . 16

2.2 Thermal Characteristics of Various Type I and II Permanent Magnets 21

3.1 GyroWheel ${ }^{\mathrm{TM}}$ Flight Model Characteristics ............. 51

4.1 Gyroscope 24 Position Test . . . . . . . . . . . . . . 76

4.2 Gyroscope 11 Position Test . . . . . . . . . . . . . 77

5.1 Temperature Sweep Time-line. . . . . . . . . . . . . . . . . 106

6.1 GyroWheel ${ }^{\mathrm{TM}}$ In-Flight Rate Sensing Algorithm Coefficients . . . . 121

A.1 15 position test: $i_{x}$ and $i_{y} \ldots \ldots \ldots$. . . . . . . . . . . . . . . . . . . . 135

A.2 15 position test: Calibration Coefficients . . . . . . . . . . 137

A.3 13(a) position test: Calibration Coefficients . . . . . . . . . . 138

A.4 13(b) position test: Calibration Coefficients . . . . . . . . . . 139

A.5 11 position test: Calibration Coefficients . . . . . . . . . . 140

A.6 8 position test: Calibration Coefficients . . . . . . . . . . . . 141 


\section{List of Figures}

2.1 Radiation Properties of Surfaces . . . . . . . . . . . . . 8

2.2 Equations for Steady-State Conduction . . . . . . . . . . . . 11

2.3 Raw Temperature Data for GyroWheel ${ }^{\mathrm{TM}} \ldots \ldots \ldots \ldots \ldots$

2.4 Fitted Temperature Data for GyroWheel ${ }^{\mathrm{TM}} \ldots \ldots \ldots \ldots \ldots$

2.5 Hysteresis Curve Showing Demagnetization Curve . . . . . . . . . 19

2.6 Magnetic Dipole Moment. . . . . . . . . . . . . . . . . . 22

2.7 Schematic Diagram of a DTG . . . . . . . . . . . 28

2.8 Gimbal Motion in a DTG . . . . . . . . . . . . . . 30

2.9 Double-Gimbaled Momentum Wheel . . . . . . . . . . . . . 35

3.1 GyroWheel $^{\mathrm{TM}}$ Exploded View $\ldots \ldots \ldots \ldots \ldots$

3.2 Gyrowheel ${ }^{\mathrm{TM}}$ Cut-away View $\ldots \ldots \ldots \ldots \ldots \ldots$

3.3 GyroWheel $^{\mathrm{TM}}$ Cross-Sectional View . . . . . . . . . . . 39

3.4 GyroWheel ${ }^{\mathrm{TM}}$ Gimbal . . . . . . . . . . . . . . . . . . . 41

3.5 Schematic Representation of GyroWheel ${ }^{\mathrm{TM}}$ Tilt Sensor Measurement 45

4.1 Mass Unbalance Error Term . . . . . . . . . . . . . . . . 65

4.2 Quadrature Error Term . . . . . . . . . . . . . . . . 67

4.3 Anisoelastic Torque . . . . . . . . . . . . . . . . . . . 67

4.4 GyroWheel ${ }^{\mathrm{TM}}$ Main Reference Frames . . . . . . . . . . 78

4.5 GyroWheel ${ }^{\mathrm{TM}}$ Additional Reference Frames . . . . . . . . . . 79

4.6 Sketch of GyroWheel ${ }^{\mathrm{TM}}$ Gimbal . . . . . . . . . . . 81

4.7 GyroWheel ${ }^{\mathrm{TM}}$ Actuator Model . . . . . . . . . . . . . . . . . 84

4.8 GyroWheel ${ }^{\mathrm{TM}}$ Misalignment Angles . . . . . . . . . . . . . 90 
5.1 GyroWheel ${ }^{\mathrm{TM}}$ Cooling Plate . . . . . . . . . . . . . . . 98

5.2 GyroWheel ${ }^{\mathrm{TM}}$ Vacuum Chamber ............... 99

5.3 GyroWheel ${ }^{\mathrm{TM}}$ Test Equipment ... . . . . . . . . . . . . . . . 100

5.4 GyroWheel $^{\text {TM }}$ Test Equipment Picture . . . . . . . . . . . . . . . 102

6.1 Average $i_{x}$ vs $T_{h, y}$ for Temperature Sweep Test and Steady State Test 115

6.2 Average $i_{y}$ vs $T_{h, x}$ for Temperature Sweep Test and Steady State Test 116

A.1 Current Values for GyroWheel ${ }^{\mathrm{TM}}$ Multi-Position Test . . . . . . . . 134

B.1 Plot of $i_{x}$ Versus $T_{h, y}$ for Multi-Position Test 1 . . . . . . . . . 143

B.2 Plot of $i_{x}$ Versus $T_{h, y}$ for Multi-Position Test $2 \ldots \ldots$. . . . . . . . 144

B.3 Plot of $i_{x}$ Versus $T_{h, y}$ for Multi-Position Test $3 \ldots \ldots$. . . . . . . 145

B.4 Plot of $i_{x}$ Versus $T_{h, y}$ for Multi-Position Test $4 \ldots$. . . . . . . 146

B.5 Plot of $i_{x}$ Versus $T_{h, y}$ for Multi-Position Test 5 . . . . . . . . . . . 147

B.6 Plot of $i_{x}$ Versus $T_{h, y}$ for Multi-Position Test $6 \ldots$. . . . . . . . 148

B.7 Plot of $i_{x}$ Versus $T_{h, y}$ for Multi-Position Test $7 \ldots$. . . . . . . . . 149

B.8 Plot of $i_{x}$ Versus $T_{h, y}$ for Multi-Position Test $8 \ldots \ldots$. . . . . 150

C.1 Plot of $B_{x}^{\prime}$ Versus $T_{h, y} \ldots \ldots \ldots \ldots$. . . . . . . . . . . 152

C.2 Plot of $B_{y}^{\prime}$ Versus $T_{h, x} \ldots \ldots \ldots \ldots$. . . . . . . . . . . . . . . . . . . .

C.3 Plot of $k_{x}^{\prime}$ Versus $T_{h, y} \ldots \ldots \ldots \ldots$. . . . . . . . . . . . 154

C.4 Plot of $k_{y}^{\prime}$ Versus $T_{h, x} \ldots \ldots \ldots \ldots$. . . . . . . . . . . . . . . . . . . . . . . . . . . . . . . . . . . . .

C.5 Plot of $c_{g, x}^{\prime}$ Versus $T_{h, y} \ldots \ldots \ldots \ldots$. . . . . . . . . . 156

C.6 Plot of $c_{g, y}^{\prime}$ Versus $T_{h, x} \ldots \ldots \ldots \ldots$. . . . . . . . . . . . . . . . . . . . .

C.7 Plot of $K_{\text {dyn }, x}^{\prime}$ Versus $T_{h, y} \ldots \ldots \ldots \ldots \ldots \ldots$

C.8 Plot of $K_{\mathrm{dyn}, y}^{\prime}$ Versus $T_{h, x} \ldots \ldots \ldots \ldots$ 


\section{Nomenclature}

\begin{tabular}{|c|c|c|}
\hline$\alpha$ & Chapter 4,6: & Torquer misalignment term for the $\mathrm{X}$ rate input axis \\
\hline$\alpha_{1}$ & $\begin{array}{l}\text { Chapter } 6,: \\
\text { Appendix A }\end{array}$ & $\begin{array}{l}\text { Misalignment angle from the } \mathrm{X} \text { rate input axis to the } \mathrm{X} \text { table } \\
\text { system axis }\end{array}$ \\
\hline$\alpha_{2}$ & $\begin{array}{l}\text { Chapter } 6,: \\
\text { Appendix A }\end{array}$ & $\begin{array}{l}\text { Misalignment angle from the } \mathrm{Y} \text { rate input axis to the } \mathrm{X} \text { table } \\
\text { system axis }\end{array}$ \\
\hline$\beta$ & Chapter 4,6: & Torquer misalignment term for the Y rate input axis \\
\hline$\beta_{1}$ & $\begin{array}{l}\text { Chapter } 6,: \\
\text { Appendix A }\end{array}$ & $\begin{array}{l}\text { Misalignment angle from the } \mathrm{X} \text { rate input axis to the } \mathrm{Y} \text { table } \\
\text { system axis }\end{array}$ \\
\hline$\beta_{2}$ & $\begin{array}{l}\text { Chapter } 6,: \\
\text { Appendix A }\end{array}$ & $\begin{array}{l}\text { Misalignment angle from the } \mathrm{Y} \text { rate input axis to the } \mathrm{Y} \text { table } \\
\text { system axis }\end{array}$ \\
\hline$\gamma_{1}$ & $\begin{array}{l}\text { Chapter } 6, \text { : } \\
\text { Appendix A }\end{array}$ & $\begin{array}{l}\text { Misalignment angle from the } \mathrm{X} \text { rate input axis to the } \mathrm{Z} \text { table } \\
\text { system axis }\end{array}$ \\
\hline$\gamma_{2}$ & $\begin{array}{l}\text { Chapter 6, : } \\
\text { Appendix A }\end{array}$ & $\begin{array}{l}\text { Misalignment angle from the } \mathrm{Y} \text { rate input axis to the } \mathrm{Z} \text { table } \\
\text { system axis }\end{array}$ \\
\hline$\delta_{n D}$ & Chapter 6: & $\begin{array}{l}\text { Angle made by the motion table system axis } n \text { and the down direction } \\
\text { for a given motion table position }\end{array}$ \\
\hline$\epsilon$ & Chapter 2: & $\begin{array}{l}\text { Emissivity, a number between } 0 \text { and } 1 \text { that is the ratio of energy } \\
\text { emitted by a substance to that emitted by a perfect radiator, or } \\
\text { blackbody, at the same temperature }\end{array}$ \\
\hline$\theta_{x}$ & Chapter 4: & $\begin{array}{l}\mathrm{X} \text { axis angle of rotation of the rotor with respect to the gimbal, } \\
\text { or the } \mathrm{x} \text { axis gimbal angle }\end{array}$ \\
\hline$\theta_{x, o}$ & Chapter 4: & Initial offset of the $x$ axis gimbal angle \\
\hline$\theta_{y}$ & Chapter 4: & $\begin{array}{l}Y \text { axis angle of rotation of the rotor with respect to the gimbal, } \\
\text { or the y axis gimbal angle }\end{array}$ \\
\hline$\theta_{z}$ & Chapter 4: & $\mathrm{Z}$ axis angle of rotation of the shaft \\
\hline$\lambda$ & Chapter 6: & Latitude \\
\hline$\mu$ & Chapter 2: & Magnetic permeability \\
\hline$\rho$ & Chapter 2: & Density \\
\hline$\sigma$ & Chapter 2: & Stefan-Boltzmann constant \\
\hline$\sigma$ & $\begin{array}{l}\text { Chapter } 5, \text { : } \\
\text { Appendix A }\end{array}$ & Standard deviation \\
\hline$\tau$ & Chapter 2,6: & Thermal time constant \\
\hline
\end{tabular}


$\phi \quad$ Chapter 6: Motion table elevation angle under a Right Hand Convention

$\phi_{i} \quad$ Chapter 3: $\quad$ GyroWheel ${ }^{\mathrm{TM}}$ rotor tilt angle around axis i

$\phi_{x} \quad$ Chapter $3,4,6,:$ Tilt, in radians, of the rotor around the GyroWheel $\mathrm{TM}$

Appendices $\quad \mathrm{x}$ axis, as provided by the tilt sensors physically located on the GyroWheel ${ }^{\mathrm{TM}}$ y axis

$\phi_{x} \quad$ Chapter 4,6: Case-referenced tilt angle of the momentum axis about the $\mathrm{x}$-axis of the case frame

$\phi_{y} \quad$ Chapter 3,4,6, : Tilt, in radians, of the rotor around the GyroWheel TM

Appendices $\quad y$ axis, as provided by the tilt sensors physically located on the GyroWheel ${ }^{T M} \mathrm{x}$ axis

$\phi_{y} \quad$ Chapter 4,6: Case-referenced tilt angle of the momentum axis about the y-axis of the case frame

$\dot{\phi}_{x} \quad$ Chapter $3,4,5,6$ : Time derivative of $\phi_{x}$ in radians per second

$\dot{\phi}_{y} \quad$ Chapter $3,4,5,6$ : Time derivative of $\phi_{y}$ in radians per second

$\psi \quad$ Chapter 6: Motion table azimuth angle under a Left Hand Convention

$\omega \quad$ Chapter 2: Angular rate of the spinning rotor

$\boldsymbol{\omega}_{c} \quad$ Chapter 4: Angular velocity vector of the spacecraft with respect to the inertial frame

$\omega_{c X} \quad$ Chapter 4,6: Angular velocity of the spacecraft with respect to the inertial frame, expressed about the $\mathrm{X}$ axis of the case frame or GyroWheel ${ }^{\mathrm{TM}}$ frame

$\omega_{c Y} \quad$ Chapter 4,6: Angular velocity of the spacecraft with respect to the inertial frame, expressed about the $\mathrm{Y}$ axis of the case frame or GyroWheel ${ }^{\mathrm{TM}}$ frame

$\omega_{e} \quad$ Chapter 6: $\quad$ Earth's rotational rate

$\omega_{g n} \quad$ Chapter 4: Gimbal angular rate about its axis n

$\omega_{\mathrm{g}} \quad$ Chapter 4:

Angular velocity vector of the GyroWheel ${ }^{\mathrm{TM}}$ gimbal

$\omega_{n} \quad$ Chapter 6 :

Components of the Earth rate as seen by the motion table system axis $\mathrm{n}$ for a given motion table position

$\omega_{r n} \quad$ Chapter 4: $\quad$ Rotor angular velocities about its axis $\mathbf{n}$

$\omega_{r} \quad$ Chapter 4: $\quad$ Complex rotor angular velocity $\left(\omega_{r}=\omega_{r x}+j \omega_{r y}\right)$

$\omega_{\mathrm{r}} \quad$ Chapter 4: Angular velocity vector of the GyroWheel ${ }^{\mathrm{TM}}$ rotor

$\omega_{s} \quad$ Chapter 2,3,4: Specific Angular rate of a spinning rotor when the system becomes tuned

$\omega_{\mathrm{S}} \quad$ Chapter 4: Angular velocity vector of the GyroWheel ${ }^{\mathrm{TM}}$ shaft

$\omega_{z} \quad$ Chapter 3,4,6, : Wheel speed around the GyroWheel $\mathrm{TM}+\mathrm{Z}$ axis, or spin axis.

Appendices

$\omega_{z} \quad$ Chapter 4: Angular velocity of the shaft about its spin axis, the case $z$ axis.

$n^{\prime} \quad$ Chapter 6, : Temperature calibrated value of variable ' $n$ ' for final GyroWheel ${ }^{T M}$

Appendix C in-flight rate sensing temperature calibration

a Chapter 4: Acceleration

$a_{N} \quad$ Chapter 4: Acceleration component along case axis $\mathrm{N}$ 


\begin{tabular}{|c|c|c|}
\hline$A$ & Chapter 2: & Area or surface area in $\mathrm{m}^{2}$ \\
\hline$A$ & Chapter 2: & $\begin{array}{l}\text { In Conduction the cross-sectional area normal to the heat transfer } \\
\text { direction }\end{array}$ \\
\hline$B$ & Chapter 2: & Magnetic Field Density \\
\hline$B_{x}$ & $\begin{array}{l}\text { Chapter } 4,6,: \\
\text { Appendix A,C }\end{array}$ & $\begin{array}{l}\text { Absolute bias or acceleration insensitive drift rate about } \\
\text { the } \mathrm{x} \text { axis }=D(x)_{F}\end{array}$ \\
\hline$B_{y}$ & $\begin{array}{l}\text { Chapter } 4,6,: \\
\text { Appendix A,C }\end{array}$ & $\begin{array}{l}\text { Absolute bias or acceleration insensitive drift rate about } \\
\text { the y axis }=D(y)_{F}\end{array}$ \\
\hline$c$ & Chapter 4: & Common damping term \\
\hline$c_{p}$ & Chapter 2: & Specific heat capacity at constant pressure \\
\hline$c_{g}$ & Chapter 4,6: & Common gimbal damping term, where $c_{g}=C_{x}=C_{y}$ \\
\hline$c_{g, x}$ & $\begin{array}{l}\text { Chapter } 4,6,: \\
\text { Appendix C }\end{array}$ & $\mathrm{X}$ axis specific damping term used in final temperature calibration \\
\hline$c_{g, y}$ & $\begin{array}{l}\text { Chapter } 4,6,: \\
\text { Appendix C }\end{array}$ & $\mathrm{Y}$ axis specific damping term used in final temperature calibration \\
\hline C & Chapter 4: & Damping matrix \\
\hline$C_{i}$ & Chapter 4: & Cosine of $i$ \\
\hline$C_{x}, C_{y}$ & Chapter 4: & Gimbal damping coefficient along the $\mathrm{x}$ and $\mathrm{y}$ axis \\
\hline$d$ & Chapter 2: & Magnetic dipole Moment \\
\hline$D(x)_{F}$ & Chapter 4: & $\begin{array}{l}\text { Acceleration insensitive drift rate about the } \mathrm{x} \text { axis, } \\
\left.\text { also called absolute bias }=B_{x} \text { ( } g \text { independent }\right)\end{array}$ \\
\hline$D(y)_{F}$ & Chapter 4: & $\begin{array}{l}\text { Acceleration insensitive drift rate about the y axis, } \\
\left.\text { also called absolute bias }=B_{y} \text { ( } g \text { independent }\right)\end{array}$ \\
\hline$D(x)_{N}$ & $\begin{array}{l}\text { Chapter } 4,6,: \\
\text { Appendix A }\end{array}$ & $\begin{array}{l}\text { Drift rate coefficient for drift rate about the } \mathrm{x} \text { axis, attributable } \\
\text { to acceleration along case axis } \mathrm{N} \text { ( } g \text { dependent) }\end{array}$ \\
\hline$D(y)_{N}$ & $\begin{array}{l}\text { Chapter } 4,6,: \\
\text { Appendix A }\end{array}$ & $\begin{array}{l}\text { Drift rate cocfficient for drift rate about the y axis, attributable } \\
\text { to acceleration along case axis } \mathrm{N} \text { ( } g \text { dependent) }\end{array}$ \\
\hline$D(x)_{M N}$ & $\begin{array}{l}\text { Chapter } 4,6,: \\
\text { Appendix A }\end{array}$ & $\begin{array}{l}\text { Drift rate coefficient for drift rate about the } \mathrm{x} \text { axis, attributable } \\
\left.\text { to acceleration along both case axis } \mathrm{M} \text { and } \mathrm{N} \text { ( } g^{2} \text { dependent }\right)\end{array}$ \\
\hline$D(y)_{M N}$ & $\begin{array}{l}\text { Chapter } 4,6,: \\
\text { Appendix A }\end{array}$ & $\begin{array}{l}\text { Drift rate coefficient for drift rate about the y axis, attributable } \\
\text { to acceleration along both case axis } M \text { and } N\left(g^{2} \text { dependent) }\right.\end{array}$ \\
\hline$F_{m}$ & Chapter 4: & Figure of Merit \\
\hline$F_{ \pm i}$ & Chapter 4: & Force vector for a torque coil located on axis i \\
\hline$h$ & Chapter 2: & Heat transfer coefficient in $\mathrm{Wm}^{-2} \mathrm{~K}^{-1}$ \\
\hline$h$ & Chapter 4: & Angular Momentum vector of a spinning wheel due to relative motion \\
\hline$h_{s}$ & Chapter 4: & $h_{s}=\left(I_{r s}+\frac{I_{g s}}{2}\right) \omega_{z}$ \\
\hline$H$ & Chapter 2: & Magnetic Field Strength \\
\hline$H$ & Chapter 2: & Angular Momentum of a spinning rotor \\
\hline$H_{\mathrm{s} / \mathrm{c}}$ & Chapter 4: & Angular Momentum vector of a spacecraft \\
\hline $\boldsymbol{H}_{\text {tot }}$ & Chapter 4: & Total Angular Momentum vector \\
\hline $\boldsymbol{H}_{\text {whee }}$ & Chapter 4: & Angular Momentum vector of a spinning wheel due to spacecraft motion \\
\hline
\end{tabular}


$i_{ \pm i} \quad$ Chapter $4: \quad$ Current in the torque coil located on axis i

$i_{x} \quad$ Chapter $3,4,6$, : Current in the torque coils physically located on the DTG or Appendix A,B GyroWheel ${ }^{\text {TM }} \mathrm{x}$ case axis

$i_{y}$ Chapter $3,4,6$, : Current in the torque coils physically located on the DTG or Appendix A GyroWheel ${ }^{\mathrm{TM}}$ y case axis

$i_{\boldsymbol{x}} \quad$ Chapter $6: \quad 11 \times 1$ vector of the 11 multi-position average $i_{x}$ currents

$i_{\boldsymbol{x}} \quad$ Chapter 6: $11 \times 1$ vector of the 11 multi-position average $i_{y}$ currents

I Chapter 2: Current

$I_{c n} \quad$ Chapter 4: Current in the axis $\mathrm{n}$ torque coil, relates to $T_{c N}$

I Chapter 2: Mass moment of inertia of the spinning rotor

$I_{g n} \quad$ Chapter 2,4: Mass moment of inertia of the gimbal about its axis $\mathrm{n}$

$I_{g s} \quad$ Chapter 4,6: Mass moment of inertia of the gimbal about the $\mathrm{z}$ axis or spin axis

$I_{g t} \quad$ Chapter 4,6 :

Ig Chapter 4:

$I_{r n} \quad$ Chapter 4 :

I $\quad$ Chapter 4:

$I_{r s} \quad$ Chapter 4,6:

$I_{r t} \quad$ Chapter 4:

$I_{t} \quad$ Chapter 4,6:

$I_{t, x} \quad$ Chapter 4,6: Gimbal transverse axis inertia when $I_{g x}=I_{g y}$

Mass moment of inertia matrix for the GyroWheel ${ }^{T M}$ gimbal Mass moment of inertia of the rotor about its axis $n$ Mass moment of inertia matrix for the GyroWheel ${ }^{\mathrm{TM}}$ rotor Mass moment of inertia of the rotor about the $\mathrm{z}$ axis or spin axis Rotor transverse axis inertia when $I_{r x}=I_{r y}$ GyroWheel $^{\text {TM }}$ transverse inertia

$I_{t, y} \quad$ Chapter 4,6 :

$\mathrm{X}$ axis specific transverse inertia term used in final temperature calibration

IS Chapter 4 : $\mathrm{Y}$ axis specific transverse inertia term used in final temperature calibration

$J \quad$ Chapter 4: $\quad J=I_{g t}-\frac{I_{g s}}{2}$

$k \quad$ Chapter 2: Thermal conductivity in $\mathrm{W} \mathrm{m}^{-1} \mathrm{~K}^{-1}$

$k 1 \quad$ Chapter 4: Scale factor vector the current of the torque coil is multiplied by to determine the force vector, $\boldsymbol{F}_{ \pm i}$

$k 2 \quad$ Chapter 4: Scale factor vector the current of the torque coil is multiplied by to determine the moment vector, $\boldsymbol{M}_{ \pm \boldsymbol{i}}$

$k_{t r q} \quad$ Chapter 4 :

$k_{\text {scale }, x} \quad$ Chapter 4 :

Scale factor the torque coil current is multiplied by to determine the gyro control torque

scale, $\infty$ Chapter $4:$

Scale factor the current, $i_{x}$, of the torque coil physically located on the $\mathrm{x}$ axis, is multiplied by to determine the sum of

$k_{\text {scale, } y} \quad$ Chapter 4: Scale factor the current, $i_{y}$, of the torque coil physically located on the y axis, is multiplied by to determine the sum of the applied input torques and error parameters about the $y$ axis

$k_{x} \quad$ Chapter 4,6, : Experimentally determined scale factor the current, $i_{x}$, of the Appendix A,C torque coil physically located on the $\mathrm{x}$ axis, is multiplied by to 
determine the sum of the applied input torques and error parameters about the $\mathrm{x}$ axis (calibrated for misalignment angles, $\alpha$ and $\beta$ )

$k_{y} \quad$ Chapter 4,6,: Experimentally determined scale factor the current, $i_{y}$, of the Appendix A,C torque coil physically located on the $\mathrm{x}$ axis, is multiplied by to determine the sum of the applied input torques and error parameters about the $\mathrm{x}$ axis (calibrated for misalignment angles, $\alpha$ and $\beta$ )

K Chapter 2,3:

$\mathrm{K}$ Chapter 4: Gyroscope flexure stiffness

$K_{x}, K_{y} \quad$ Chapter 2,4 :

$K_{\text {dyn }}$

Chapter 4,6:

Stiffness matrix

$K_{\mathrm{dyn}, x}$

Chapter 4,6, :

Gyroscope flexure stiffness along the $\mathrm{x}$ and $\mathrm{y}$ axis

Dynamic stiffness term, $K_{\mathrm{dyn}}=\frac{K_{x}+K_{y}}{2}-J \omega_{z}^{2}$

Appendix C

$\mathrm{X}$ axis specific dynamic stiffness term used in final temperature

$K_{\text {dyn }, y}$

Chapter 4,6, : calibration

Appendix $\mathrm{C}$

$\mathrm{Y}$ axis specific dynamic stiffness term used in final temperature calibration

$m \quad$ Chapter 2:

$m \quad$ Chapter 4 :

$m_{1}, m_{2}$ Chapter 4 :

$M \quad$ Chapter 2:

Magnetic Moment

Mass

Mass inertia coefficients

Torque applied normal to the angular momentum vector of a spinning rotor

M. Chapter 4:

$M_{x} \quad$ Chapter 4 :

Mass inertia matrix

$M_{y} \quad$ Chapter 4:

Moment acting about the $\mathrm{x}$ axis of the rotor in a non spinning coordinate system

Moment acting about the $y$ axis of the rotor in a non spinning coordinate system

$M_{r n} \quad$ Chapter 4:

Moment acting about axis $\mathrm{n}$ of the rotor

$M_{ \pm i} \quad$ Chapter 4 :

$M U_{x} \quad$ Chapter 4:

$M U_{y} \quad$ Chapter 4:

$n \quad$ Chapter 2:

$N \quad$ Chapter 2:

$q \quad$ Chapter 4:

$Q \quad$ Chapter 2:

$\dot{Q} \quad$ Chapter 2:

$\dot{Q}_{A} \quad$ Chapter 2:

$Q_{x} \quad$ Chapter 4:

$Q_{y} \quad$ Chapter 4:

Force vector for a torque coil located on axis $\mathrm{i}$

Mass unbalance of axis $\mathrm{x}=D(x)_{X}$

Mass unbalance of axis $\mathrm{y}=D(y)_{Y}$

Unit vector normal to the plane of an electromagnetic wire loop

Number of equal turns or loops of an electromagnetic wire

Vector of gimbal angles, $\theta_{x}, \theta_{y}$ and the shaft rotation angle, $\theta_{z}$

Energy heat transfer

Rate of heat transfer or flow in W

Rate of heat transfor or flow in $\mathrm{W}$ per unit, area

Quadrature of axis $\mathrm{x}=D(x)_{Y}$

Quadrature of axis $\mathrm{y}=D(y)_{X}$

$r_{ \pm i} \quad$ Chapter 4 :

Distance along axis i from each of the four GyroWheel $\mathrm{TM}_{\text {torque coils }}$ to the origin of the gimbal frame

$S_{i} \quad$ Chapter $4: \quad$ Sine of $i$

$t \quad$ Chapter 2,4: Time

$T \quad$ Chapter 2: Temperature 


\begin{tabular}{|c|c|c|}
\hline$T$ & Chapter 4: & Torque \\
\hline$T$ & Chapter 4: & Total kinetic energy \\
\hline$T_{c}$ & Chapter 2: & $\begin{array}{l}\text { Curie Temperature, Temperature at which a magnetic material loses } \\
\text { all magnetic properties }\end{array}$ \\
\hline$T_{c}$ & Chapter 4: & Case referenced vector of GyroWheel ${ }^{\mathrm{TM}}$ control torques \\
\hline$T_{c X}$ & Chapter 4: & $\begin{array}{l}\text { Case referenced control torque acting about the } \mathrm{X} \text { axis of the case } \\
\text { frame or GyroWheel } \\
\text { TM frame }\end{array}$ \\
\hline$T_{c Y}$ & Chapter 4: & $\begin{array}{l}\text { Case referenced control torque acting about the } \mathrm{Y} \text { axis of the case } \\
\text { frame or GyroWheel }{ }^{\mathrm{TM}} \text { frame }\end{array}$ \\
\hline$T_{c Z}$ & Chapter 4: & $\begin{array}{l}\text { Case referenced control torque acting about the } \mathrm{Z} \text { axis of the case } \\
\text { frame or GyroWheel }\end{array}$ \\
\hline$T_{\text {ext }}$ & Chapter 4: & Vector of external torques \\
\hline$T_{f}$ & Chapter 2: & Fluid temperature for convection \\
\hline$T_{g n}$ & Chapter 4: & Generalized control torques acting about gimbal axis $n$ \\
\hline$T_{h, x}$ & $\begin{array}{l}\text { Chapter } 3,6,: \\
\text { Appendix C }\end{array}$ & $\begin{array}{l}\text { Housing temperature for the GyroWheel }{ }^{\mathrm{TM}} \mathrm{x} \text { case axis in degrees } \\
\text { Celsius. }\end{array}$ \\
\hline$T_{h, y}$ & $\begin{array}{l}\text { Chapter } 3,6,: \\
\text { Appendix B,C }\end{array}$ & $\begin{array}{l}\text { Housing temperature for the GyroWheel }{ }^{\mathrm{TM}} \text { y case axis in degrees } \\
\text { Celsius. }\end{array}$ \\
\hline$T_{o}$ & Chapter 2: & Initial temperature \\
\hline$T_{r n}$ & Chapter 4: & $\begin{array}{l}\text { Control torques acting on the rotor, expressed in axis } \mathrm{n} \text { of the } \\
\text { body frame }\end{array}$ \\
\hline$T_{r}$ & Chapter 4: & Complex control torque $\left(T_{r}=T_{r x}+j T_{r y}\right)$ \\
\hline$T_{\infty}$ & Chapter 2: & $\begin{array}{l}\text { Final temperature a lumped parameter heat transfer system is } \\
\text { stabilizing to }\end{array}$ \\
\hline$V$ & Chapter 2: & Volume \\
\hline$V$ & Chapter 4: & Total potential energy \\
\hline$V D$ & Chapter 3: & $\begin{array}{l}\text { Time between vertical line to next vertical line on the GyroWheel } \\
\text { rotor triangular pattern for optical tilt sensing }\end{array}$ \\
\hline$V V$ & Chapter 3: & $\begin{array}{l}\text { Time between vertical line to next diagonal line on the GyroWheel } \mathrm{TM} \\
\text { rotor triangular pattern for optical tilt sensing }\end{array}$ \\
\hline$\Delta x$ & Chapter 2: & Length of the heat transfer path \\
\hline$x, y$ & Chapter 4: & Rotor axes (non-rotating) in a plane normal to the spin axis, z \\
\hline $\boldsymbol{X}_{\boldsymbol{x}}$ & Chapter 6: & $10 \times 1$ vector of DTG ground calibration error terms used in SVD \\
\hline$X_{y}$ & Chapter 6: & $10 \times 1$ vector of DTG ground calibration error terms used in SVD \\
\hline & Chapter 4: & Rotor spin axis \\
\hline$X, Y, Z$ & Chapter 4: & Gyro case axes \\
\hline
\end{tabular}




\section{Chapter 1}

\section{Introduction}

\subsection{Thesis Overview}

Placing objects into space can be fundamentally worked down to a dollar figure for each kilogram of mass placed into orbit. Ultimately satellite designers are looking for the lightest possible equipment capable of providing them with the reliability, accuracy, and functionality required for their design. It inherently makes sense that satellite designers would like to see necessary systems combined where possible into a lighter, cheaper, smaller, commercially available and proven system. The Bristol GyroWheel ${ }^{T M}$ is one such system.

It is an innovative spacecraft attitude determination and control system (ADCS) device that provides both an angular momentum bias and control torques about three axes while also measuring the spacecraft angular rates about the two axes perpendicular to the spin direction. Hence, this device is both an actuator and a sensor at the same time. The use of one lightweight mechanism to provide 3-axis actuation and 2-axis rate sensing is unique in the field of spacecraft attitude control.

As stated above, satellite designers wish to combine systems in order to utilize the lightest possible equipment while also getting the accuracy they require. The multi- 
function capability of the GyroWheel ${ }^{T M}$ allows for implementing an ADCS design with a significant reduction in the number of ACS components that are required to achieve the same level of fine pointing performance. Specifically this thesis details: the achievement of greater accuracy in the performance of the GyroWheel ${ }^{\mathrm{TM}}{ }_{\text {system }}$ through calibration of the system to compensate for changes in the magnetic field strength (B field) due to temperature, making it so that this ADCS system is not required to be part of a satellites active thermal control system; and the addition of greater functionality of the GyroWheel ${ }^{\mathrm{TM}}$ system through calibration of the 2-axis rate sensing for de-tuned conditions involving changes in both the wheel speed and rotor plane tilt from the null position.

\subsection{Motivation For Testing}

In an effort to improve the accuracy of Bristol's GyroWheel ${ }^{T M}$ product beyond the already specified and achieved design value, add additional ADCS functionality, and to further the knowledge base in this area of research and this system, Carleton University and Bristol undertook the testing required to calibrate the flight model of the GyroWheel ${ }^{\mathrm{TM}}$, which was to be utilized on the SCISAT-1 small scientific satellite developed for the Canadian Space Agency. Testing was carried out using facilities at the inertial navigation laboratory of Defence Research and Development Centre Ottawa at Shirley's Bay.

Given that the actual GyroWheel ${ }^{\text {TM }}$ flight model to be used in the SCISAT-1 small satellite was available for testing for only a short period of time due to satellite integration and SCISAT-1 project schedule, the testing was conducted very aggressively on a 24 hour a day schedule from the $26^{\text {th }}$ of November 2002 until the $27^{\text {th }}$ of March 2003. 


\subsection{Thesis Outline}

The scope of this thesis is to provide the background, methodology, analysis and results leading to the improvement of the accuracy of the GyroWheel ${ }^{\mathrm{TM}}$ for changes in temperature and the addition of de-tuned rate sensing capability.

Chapter 2 is an overview of the concepts that are utilized including: satellite thermal control subsystems; the changes of magnetic field strength with temperature; the use of gyro devices in satellite inertial navigation; and satellite momentum management.

Chapter 3 discusses the construction of the GyroWheel ${ }^{\mathrm{TM}}$ highlighting the uniqueness of the system and listing the measured variables that are provided by the GyroWheel ${ }^{T M}$. These are elaborated on in Chapter 4 when discussing the derivation of the calibration equations and the error calibration terms.

Chapter 4 begins with the equations required for in-flight rate sensing and ground calibration for a Dynamically Tuned Gyroscope (DTG). The similarities and uniqueness of the GyroWheel ${ }^{T M}$ system when compared to a DTG are then discussed along with the relation to the in-flight rate sensing and ground calibration for temperature and de-tuned conditions, including the additional terms required due to the presence of gravitational acceleration. The equations are then manipulated to suit the testing environment in order to facilitate the calculation of the error calibration terms given the variables provided by the GyroWheel ${ }^{\mathrm{TM}}$ as discussed in Chapter 3 .

Chapter 5 begins by discussing the specific equipment used in the testing for the calibration of the GyroWheel ${ }^{\mathrm{TM}}$ system. This equipment is highly specialized and its use is discussed in detail including any limitations of the equipment. References are made to the theory discussed in Chapter 2. The specific tests conducted arc then discussed along with the motivation and end product for each test. References are made to the equations developed in Chapter 4.

Chapter 6 presents the analysis and final results from testing as well as draws 
conclusions about the calibration process. The findings are discussed and the determination of the manner to apply the temperature and de-tuned calibration is rationalized and defined. 


\section{Chapter 2}

\section{Overview of Spacecraft Systems}

Over the past four decades the engineering design of spacecraft has evolved from infancy to well-defined techniques supported by analysis tools, manufacturing technology, and space qualified hardware. In gencral, after the spacecraft mission and payload have been determined, the functions required to support the spacecraft can be summarized as follows: support the payload mass; point the payload correctly; keep the payload at the right temperature; provide electric power, commands, and telemetry; put the payload in the right orbit and keep it there; and provide data storage and communications if required. The spacecraft consists of subsystems or equipment groups which provide these functions.

As the GyroWheel ${ }^{\mathrm{TM}}$ is an innovative Attitude Determination and Control System, this thesis deals mainly with the spacecraft ADCS subsystem (specifically focusing on inertial navigation utilizing gyro devices), but also touches on the Thermal subsystem since the GyroWheel ${ }^{\mathrm{TM}}$ temperature is not being actively controlled under the spacecraft thermal subsystem. Therefore, the GyroWheel ${ }^{\mathrm{TM}}$ rate sensing capabilities must be calibrated for the temperature range it will be subjected to in order to improve accuracy. 


\subsection{Satellite Thermal Control Systems and Heat Transfer}

The thermal subsystem controls the spacecraft equipment's temperatures. It does so by the physical arrangement of equipment and using thermal insulation and coatings to balance, or transport and reject, heat from power dissipation in components, absorption from the Earth and Sun, and radiation to space. Generally the temperature compartments for conventional electronics can be controlled passively by coating or insulating their outer surfaces. These coatings are selected to provide a balance between heat absorbed and heat radiated to space. Sometimes passive, thermal-balance techniques are not enough and more extensive active thermal control is required.

As discussed by Wertz and Larson [28], it has been established that components with stringent temperature requirements or components that dissipate large amounts of heat due to their electrical power usage, require more precise thermal control. For example gyros and precision oscillators are often placed in insulated compartments, with active electrical heaters to control temperatures. Traveling wave tubes and other elements that dissipate a lot of power concentrate their dissipation locally. This often results in hot spots. Heat is normally conducted away from these hot spots and spread over a thermal panel where it radiates to space. Thus, intense hot spots and equipment that must meet tight thermal limits will require the use of high-capacity heat conductors, i.e. heat pipes, to actively move the heat and equalize temperatures. The amount of heat dissipation and temperatures required for equipment to operate determine the subsystem's size. Most power for thermal control is used in heaters to keep components from getting too cold.

In most instances the heat inputs on the spacecraft are highly variable with time. For example, as stated in Wertz and Larson [28], "the mean value of direct solar heating on a spacecraft surface varies from $1367 \mathrm{~W} \mathrm{~m}^{-2}$ when the Sun is normal to 
the surface, to zero when the spacecraft is in eclipse."

\subsubsection{Principles of Heat Transfer}

As discussed by Wertz and Larson [28], heat is an energy form that has the capacity to flow or diffuse from bodies of high heat energy to bodies of low heat energy. When heat is flowing at a continuous or uniform rate heat transfer is said to be in a steady state. However, this is often not the case. In general, heat transfer can be divided into three major areas: radiation, convection, and conduction. Radiation is energy transfer via electromagnetic waves; convection is thermal energy transfer between a flowing fluid and a solid interface; and conduction is thermal energy transfer through matter in the absence of fluid motion.

\section{Radiation}

All matter radiates electromagnetic energy with the total radiated energy per unit time per unit surface area, $\dot{Q}_{A}$, given by the Stefan-Boltzmann equation:

$$
\dot{Q}_{A}=\epsilon \sigma T^{4}
$$

where $\sigma$ is the Stefan-Boltzmann constant $\left(5.67051 \times 10^{-8} \mathrm{~W} \mathrm{~m}^{-2} \mathrm{~K}^{-4}\right), T$ is the absolute temperature, and $\epsilon$ is the emissivity. Emissivity is defined as the ratio of energy emitted by a substance to that emitted by a perfect radiator, or blackbody, at the same temperature.

As shown in Figure 2.1, Wertz and Larson [28] describe surface radiation properties, in addition to emissivity. In summary, a transparent plate's surface will reflect, absorb, or transmit incoming radiation when irradiated. The percentage of incoming radiation absorbed is defined as absorptivity, the percentage reflected as reflectivity. The percentage transmitted is defined as transmissivity. They are expressed as fractions of the total irradiance, and given these are the three only outcomes, their sum must equal 1.0. 


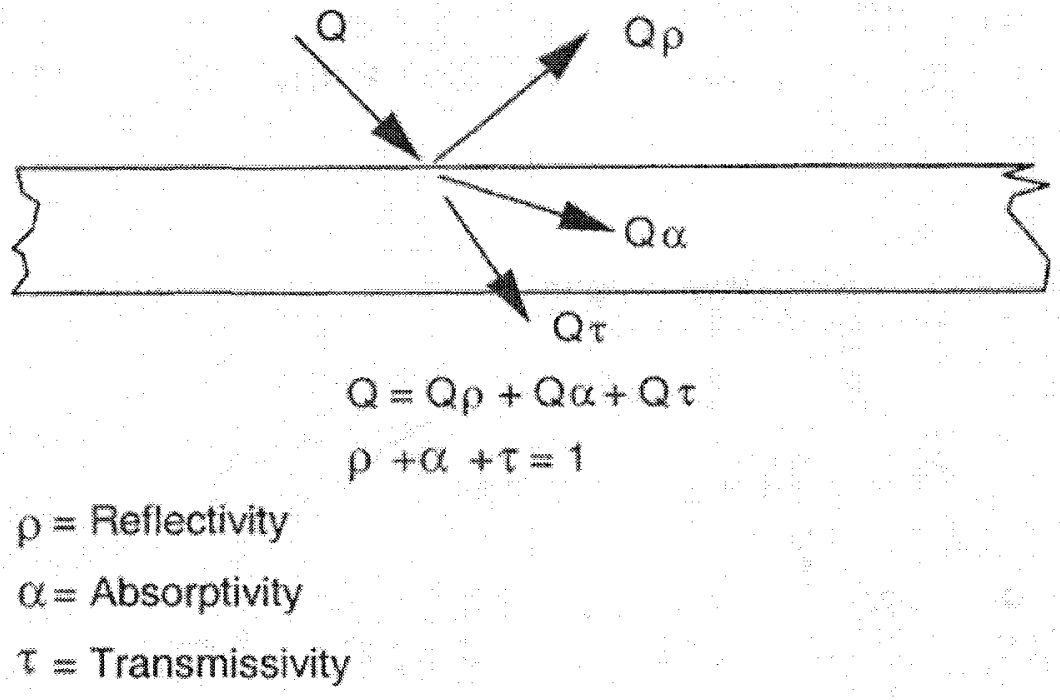

Figure 2.1: Radiation Properties of Surfaces, from Wertz and Larson [28]. Any surface irradiated by electromagnetic radiation absorbs it $(\alpha)$, reflects it $(\rho)$, or transmits it $(\tau)$. 
Diffuse gray surface conditions are generally assumed for heat transfer that occurs between two bodies. To be defined as diffuse, two conditions must be met. First, reflected and emitted radiation must follow Lambert's cosine law and second, gray surfaces must have emissivity and absorptivity that are independent of wavelength.

The following equations adapted from Wertz and Larson [28] are generally used to compute the energy exchange rate, $\dot{Q}$ in W, from one body to the other body, in a nonabsorbing medium. For a gray surface surrounded by deep space:

$$
\dot{Q}=\sigma \epsilon_{1} A_{1}\left(T_{1}^{4}-T_{2}^{4}\right)
$$

because $T_{\text {deep space }}=4 \mathrm{~K}$, we generally assume $T_{2}^{4}=0$. For two flat surfaces short distances apart:

$$
\dot{Q}=\frac{1}{\left(\frac{1}{\epsilon_{1}}+\frac{1}{\epsilon_{2}}-1\right)} \sigma A_{1}\left(T_{1}^{4}-T_{2}^{4}\right)
$$

For coaxial long cylinders and concentric spheres:

$$
\dot{Q}=\frac{1}{\left(\frac{1}{\epsilon_{1}}+\frac{A_{1}}{A_{2}}\left(\frac{1}{\epsilon_{2}}-1\right)\right)} \sigma A_{1}\left(T_{1}^{4}-T_{2}^{4}\right)
$$

The equations given above define $\sigma$ as the Stefan-Boltzmann constant, $A$ as the area in $\mathrm{m}^{2}, \epsilon$ as emissivity, and $T$ as temperature in K. In Equation 2.4, subscript 1 refers to inside surface and 2 to outside surface.

\section{Convection}

Convection occuring between a solid surface and its adjacent fluid is described by:

$$
\dot{Q}=h A \triangle T
$$

where $\dot{Q}$ is the rate of heat flow in $\mathrm{W}, h$ is the heat transfer coefficient in $\mathrm{W} \mathrm{m}^{-2} \mathrm{~K}^{-1}$. $A$ is the surface area in $\mathrm{m}^{2}, \Delta T$ is the temperature difference between the surface and the fluid mass in K. The heat transfer cocfficient $h$ is dependant upon the 
geometry of the surface in addition to the fluid's flow characteristics and its physical properties. It is of importance to note that determining $h$ is a central problem of convective heat-transfer analysis, but because of the vacuum in space, convection does not play a large role in the thermal control of non-manned spacecraft.

\section{Conduction}

The fundamental equation for steady-state, one-dimensional heat conduction in rectangular coordinates, as presented by Wertz and Larson [28] is:

$$
\dot{Q}=(k A / \Delta x)\left(T_{1}-T_{2}\right)
$$

where $\dot{Q}$ in $\mathrm{W}$ is the energy transfer rate, $T$, in $\mathrm{K}$, is the temperature, $k$, in $\mathrm{W} \mathrm{m}^{-1} \mathrm{~K}^{-1}$, is the thermal conductivity, $\Delta x$, in $\mathrm{m}$, is the length of the heat transfer path, and $A$, in $\mathrm{m}^{2}$, is the cross-sectional area normal to the heat transfer direction. Figure 2.2 from Wertz and Larson [28] illustrates steady-state conduction for rectangular, cylindrical, and spherical coordinate systems and is presented below.

\subsubsection{Thermal Time Constant}

When a body is being cooled or heated by convection or radiation and the thermal conductivity is large, such that rapid heat transfer rates within the body allow for the assumption that the body temperature distribution is uniform, then the situation is considered to be a lumped capacity or lumped parameter system. In this type of system, the complex methods of transient heat transfer are not required and a simple energy balance equation may be drawn up and integrated. The most common case is quenching, a convective boundary problem for which in time, $\mathrm{d} t$, a small heat transfer, $\dot{Q}$, occurs when the body temperature changes from $T$ by an amount $\mathrm{d} T$. Thus:

$$
\dot{Q}=\rho c_{p} V \frac{\mathrm{d} T}{\mathrm{~d} t}=-h A\left(T-T_{f}\right)
$$




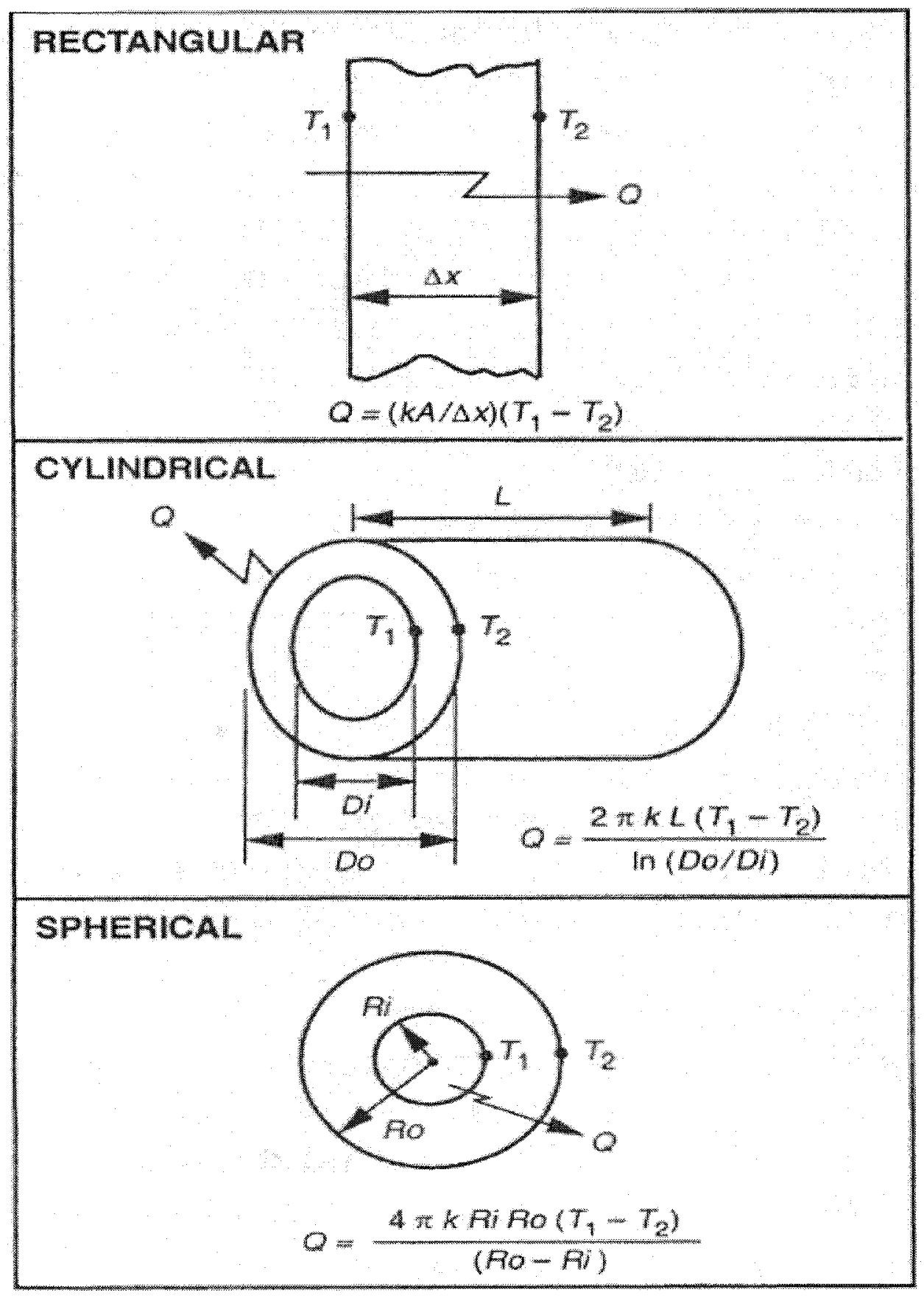

Figure 2.2: Equations for Steady-State Conduction, from Wertz and Larson [28]. These equations allow for the computation of the amount of heat, $Q$, conducted through each shape, depending on the thermal conductivity, $k$, of the material, and the other variables. 
where $T_{f}$ is the fluid temperature, $A$ is the body surface area, $V$ is the body volume, $\rho$ is the body density, $c_{p}$ is the body specific heat, and $h$ is the heat transfer coefficient. Integration gives:

$$
\frac{\left[T_{f}-T(t)\right]}{\left[T_{f}-T_{o}\right]}=\mathrm{e}^{-\left(\frac{h A t}{\rho c_{p} V}\right)}
$$

where $T_{o}$ is the initial temperature and $T(t)$ is the temperature at any future time $t$. The thermal time constant of the system can therefore be defined as:

$$
\tau=\frac{\rho c_{p} V}{h A}
$$

and Equation 2.9 can be rewritten as:

$$
\frac{\left[T_{f}-T(t)\right]}{\left[T_{f}-T_{o}\right]}=\mathrm{e}^{-\left(\frac{t}{\tau}\right)}
$$

In space, this concept of a thermal time constant also applies, but only when it can still be assumed that the spacecraft thermal conductivity is comparatively large such that the rapid heat transfer rates within the body enable uniform body temperature distribution to be assumed. In the case of quenching, the amount of energy dissipated is due solely to convection and not radiation. However, in the case of orbiting spacecraft it is due solely to radiation. Thus Equation 2.9, calculated based on convection, does not apply as different variables must be utilized to calculate the thermal time constant. However, Equation 2.10 still applies; $T_{f}$, the fluid temperature is changed to $T_{\infty}$, or the final temperature that the system is stabilizing to, making Equation 2.10:

$$
\frac{\left[T_{\infty}-T(t)\right]}{\left[T_{\infty}-T_{o}\right]}=\mathrm{e}^{-\left(\frac{t}{\tau}\right)}
$$

This can be rewritten as:

$$
T(t)=T_{\infty}+\left(T_{o}-T_{\infty}\right) \mathrm{e}^{-\left(\frac{t}{\tau}\right)}
$$

\section{GyroWheel $^{\mathrm{TM}}$ Thermal Time Constant}

Detailed mathematical modeling of the GyroWheel ${ }^{\mathrm{TM}}$ has not been carried out and is outside the scope of this thesis. Instead a lumped parameter model as discussed 
above has been assumed that uses simple exponential transition from an initial temperature to a final temperature and the time-temperature profile is assumed to be as per Equation 2.12.

A summary of the determination of the thermal time constant for the GyroWheel TM by Schultz [20] is presented below. Temperature profiles were taken simultaneously from the GyroWheel ${ }^{T M}$, during a test period of just over 2 hours, at three different positions: the GyroWheel ${ }^{\mathrm{TM}}$ Case Housing; the Spin motor; and the Bearing. All temperature sensors utilized on GyroWheel ${ }^{\mathrm{TM}}$ are thermistors, model YSI 46006. The temperature unit recorded by the GyroWheel ${ }^{\mathrm{TM}}$ is the "count", which is the weighted integer for telemetry produced from the GyroWheel ${ }^{T M}$ resident temperature calculation. The conversion relationship between engineering units (EU), in degrees Celsius, and the raw telemetry value (RTV), in counts, is a linear relationship such that:

$$
\mathrm{EU}=\mathrm{RTV} \times \frac{\mathrm{EU}}{\text { Bit Value }}+\mathrm{Min} \text { Value }
$$

where EU/Bit Value (in degrees Celsius per count) and MinValue (in degrees Celcius) are table uploaded values to the GyroWheel ${ }^{\mathrm{TM}}$ digital signal processor. During testing the GyroWheel ${ }^{\mathrm{TM}}$ was not enclosed in the environmental housing and the ambient lab temperature was $21.6{ }^{\circ} \mathrm{C}$.

The time temperature profiles allow for the quick calculation of thermal time constants for the GyroWheel ${ }^{\mathrm{TM}}$ by fitting the data to the assumed mathematical model of the thermal response of the GyroWheel ${ }^{\mathrm{TM}}$. A direct fit of the data to Equation 2.12 by "plotting the logarithms" can be performed only with difficulty, due to the unknown final temperature and the exponential nature of the relationship. To illustrate this point Equation 2.12 can be written in the form:

$$
\ln \left(T(t)-T_{\infty}\right)=\ln \left(\left(T_{o}-T_{\infty}\right) \mathrm{e}^{-\left(\frac{t}{\tau}\right)}\right)
$$

and finally in the form:

$$
\ln \left(T(t)-T_{\infty}\right)=\ln \left(T_{o}-T_{\infty}\right)-\frac{t}{\tau}
$$


Equation 2.15 is now in the form $y=m x+b$ where $y=\ln \left(T(t)-T_{\infty}\right), m=-1 / \tau$, and $b=\ln \left(T_{o}-T_{\infty}\right)$. "Plotting the logarithms", meaning plotting $\ln \left(T(t)-T_{\infty}\right)$ versus time requires a good knowledge of $T_{\infty}$ which is not available in this instance. An easier method can produce the time constant by using statistically determined rates of temperature change as outlined below.

Differentiating Equation 2.12 with respect to time yields:

$$
\frac{\mathrm{d} T}{\mathrm{~d} t}=\frac{T_{\infty}-T_{0}}{\tau} \mathrm{e}^{-\left(\frac{t}{\tau}\right)}
$$

Dividing Equation 2.16 at time zero, $t_{o}=0$, by Equation 2.16 evaluated at some other time prior to reaching steady state, $t_{1}$, where $t_{0}<t_{1}<\infty$, yields:

$$
\frac{(\mathrm{d} T / \mathrm{d} t)_{t_{0}}}{(\mathrm{~d} T / \mathrm{d} t)_{t_{1}}}=\mathrm{e}^{-\left(\frac{\left(t_{1}-t_{0}\right)}{\tau}\right)}=\mathrm{e}^{-\left(\frac{t_{1}}{\tau}\right)}
$$

After estimating the two rates $(\mathrm{d} T / \mathrm{d} t)_{t_{0}}$ and $(\mathrm{d} T / \mathrm{d} t)_{t_{1}}$, Equation 2.17 allows the direct estimation of the time constant, $\tau$, since $t_{1}$ is known through the process of estimating $(\mathrm{d} T / \mathrm{d} t)_{t_{1}}$.

Figure 2.3, from Schultz [20], shows a time plot of the three measured raw temperature data streams. Both the Case Housing and Bearing temperatures show initial transients that lie outside the general shape of Equation 2.12; these transients can not be included in statistical processing to obtain rates and, so, the initial 750 seconds of the data set was cropped from the database. MatLab TM version 12 was used with these reduced data sets to determine 6th and 7th order polynomial fits to the data. From the first time derivative of these polynomials the initial rates of change and the final rates of change were found, to which Equation 2.17 was applied to determine the time constants.

Once the time constants are determined the final temperature can be estimated. Rearranging Equation 2.12, specifically evaluated at time $t_{1}$ yields:

$$
T_{\infty}=\frac{T_{0} \mathrm{e}^{\left(\frac{t_{1}}{\tau}\right)}-T\left(t_{1}\right)}{\mathrm{e}^{\left(\frac{t_{1}}{\tau}\right)}-1}
$$




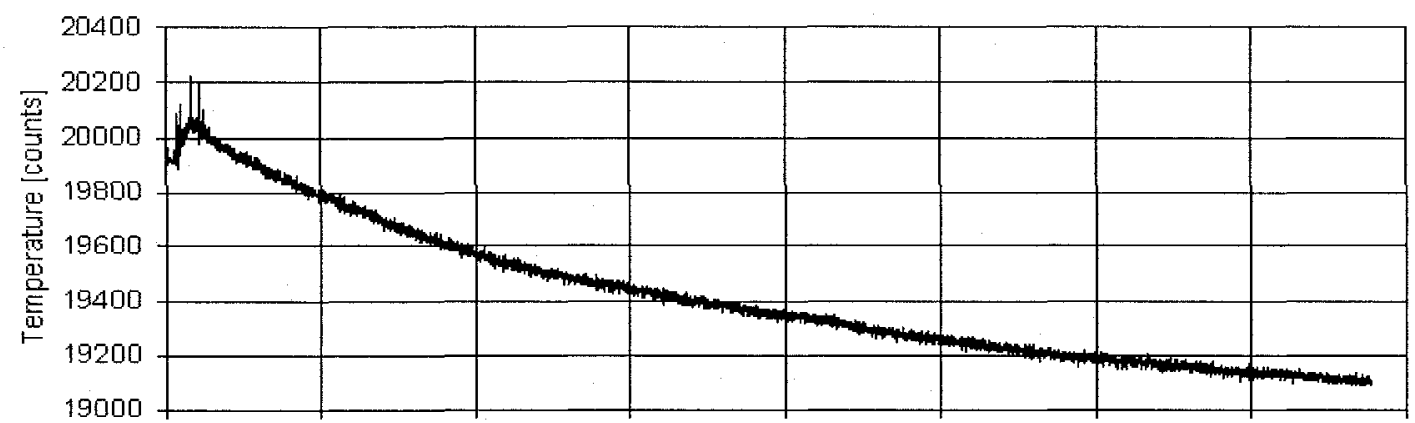

(a)

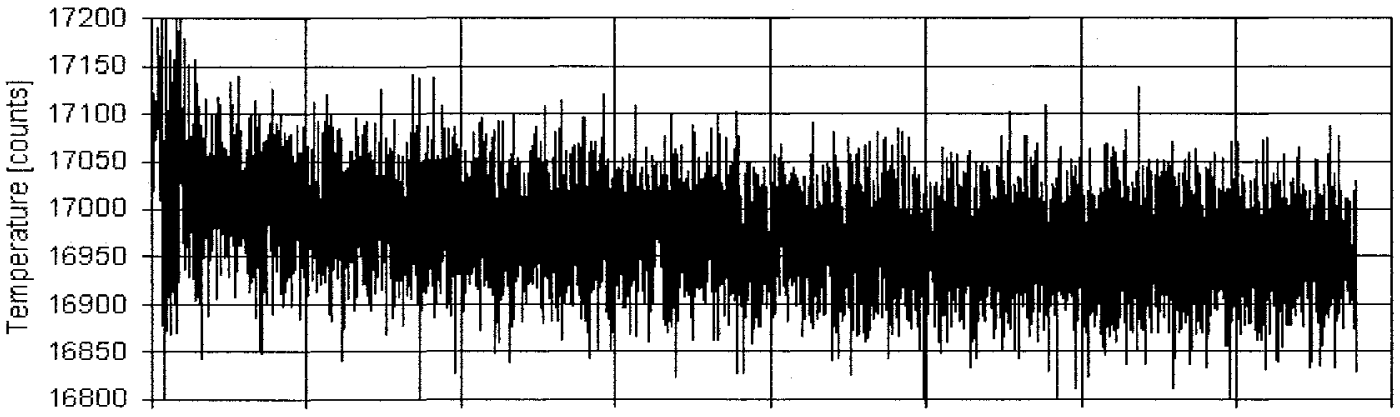

(b)

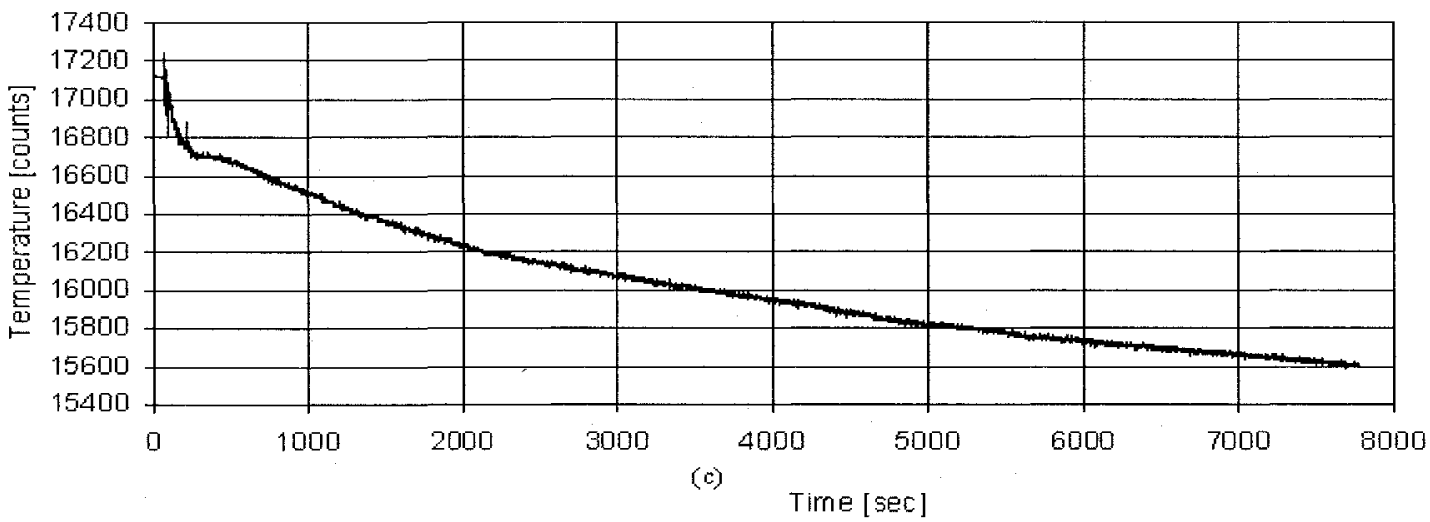

Figure 2.3: Raw temperature data for GyroWheel ${ }^{\mathrm{TM}}$ from Schultz [20]: (a) Case Housing Temperature, (b) Spin Motor Temperature, (c) Bearing Temperature 
Table 2.1: Estimated parameters from Schultz [20] for Equation 2.12 based on analysis of raw temperature data using a 6 th and 7 th order polynomial fit to cropped data.

\begin{tabular}{|l|c|c|c|c|}
\hline Data stream & $\begin{array}{c}T_{0} \\
{[\text { counts] }}\end{array}$ & $\begin{array}{c}T\left(t_{1}\right) \\
{[\text { counts] }}\end{array}$ & $\begin{array}{c}\tau \\
{[\text { minutes] }]}\end{array}$ & $\begin{array}{c}T_{\infty} \\
{[\text { counts] }}\end{array}$ \\
\hline $\begin{array}{l}\text { Case Housing temp } \\
\text { (7th order fit) }\end{array}$ & 19842 & 19102 & 71.9 & 18919 \\
\hline $\begin{array}{l}\text { Spin motor temp } \\
\text { (6th order fit) }\end{array}$ & 17010 & 16951 & 65.2 & 16940 \\
\hline $\begin{array}{l}\text { Bearing temp } \\
\text { (7th order fit) }\end{array}$ & 16581 & 15606 & 64.9 & 15411 \\
\hline
\end{tabular}

The estimated values for $T_{0}$ and $T\left(t_{1}\right)$, and the calculated values for $\tau$ and $T_{\infty}$ are shown in Table 2.1. Figure 2.4 ,from Schultz [20], shows the cropped data stream and the fitted curves based on the analysis discussed.

Therefore, the thermal time constant of the GyroWheel ${ }^{\mathrm{TM}}$ is estimated to be $65-72$ minutes. The variation in the thermal time constants from three different temperature sources on the GyroWheel ${ }^{\mathrm{TM}}$ account for this wide range.

\subsection{Magnetic Field Changes with Temperature}

Various components critical to rate sensing in typical Dynamically Tuned Gyroscopes (DTGs) are sensitive to temperature variation, which could lead to inaccurate readings. These include field strength of the permanent magnets and resistor values in torque coil current sensing circuitry. To provide the best rate sensing capability DTGs have been temperature controlled to minimize variation in these components. The GyroWheel ${ }^{\mathrm{TM}}$ is not temperature controlled and therefore needs to be calibrated for these magnetic variations due to temperature. 


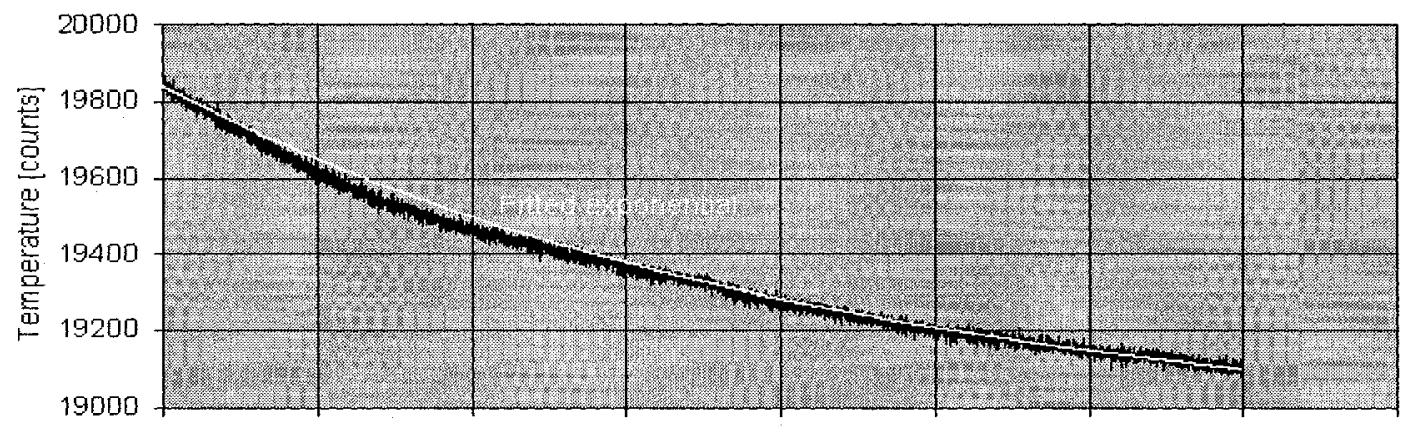

(a)

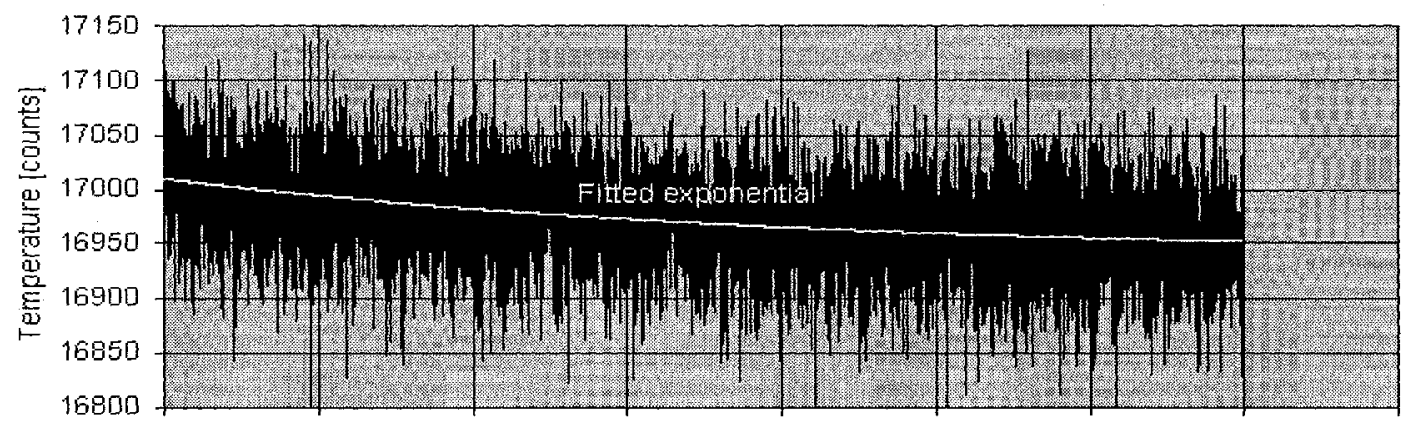

(b)

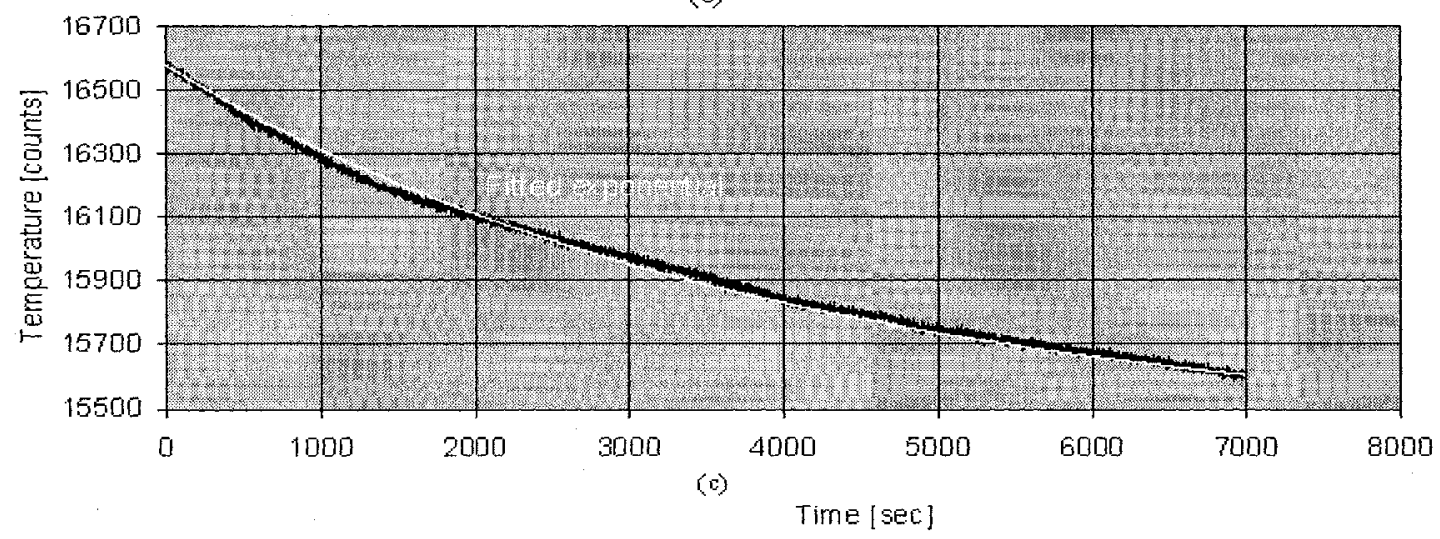

Figure 2.4: Cropped data (black) with fitted exponential curve (white), from Schultz [20], based on Table 2.1 values for: (a) Case Housing Temperature, (b) Spin Motor Temperature, (c) Bearing Temperature 


\subsubsection{Permanent Magnet Field Strength}

As discussed by Wiktowy [30], permanent magnets are materials that contain residual magnetic fields after an external field is removed. When no cxternal field is applied, the magnetic characteristics (Magnetic field strength, $H$ and Magnetic field density, $B$ ) remain in the demagnetization area (second quadrant) of the material hysteresis curve as shown in Figure 2.5 from Wiktowy [30], where:

$$
B=\mu H
$$

Type I and Type II are two general classifications of permanent magnets that each represent magnetic material at the two extremes of magnetic characteristics.

Type I permanent magnets, also referred to as soft magnetic material, share several common characteristics. The demagnetization curve of a type 1 magnet is non-linear. Thus the type I Magnet is sensitive to a demagnetizing field and may lose field strength if the demagnetizing field is high enough. In general, type I magnets have a relative magnetic permeability that is greater than 1 . This characteristic therefore allows a lot of leakage flux to occur and in some cases only $45 \%$ of the flux reaches the end of the magnet as stated by Parker [18]. As a result, type I magnets store the bulk of their energy in the leakage flux where it is difficult to utilize. Magnets of this type, including Magnetized Iron, Magnetized Steel and Alnico (an alloy of Aluminum, Nickel and Cobalt), can be machined easily and manipulated into very complex shapes. Type I magnets can also withstand physical impacts.

In contrast, the demagnetization curve (portion of the hysteresis curve in the second quadrant shown in Figure 2.5) of a type II magnet, also referred to as hard magnetic material, is relatively linear. This linearity extends into the third quadrant of the hysteresis curve. The end result is that type II magnets are very resistant to demagnetizing ficlds and do not readily lose ficld strength. The relative magnetic permeability of type II magnets is very close to 1 . This results in most of the flux reaching the end of the magnet and very little leakage flux along the length 


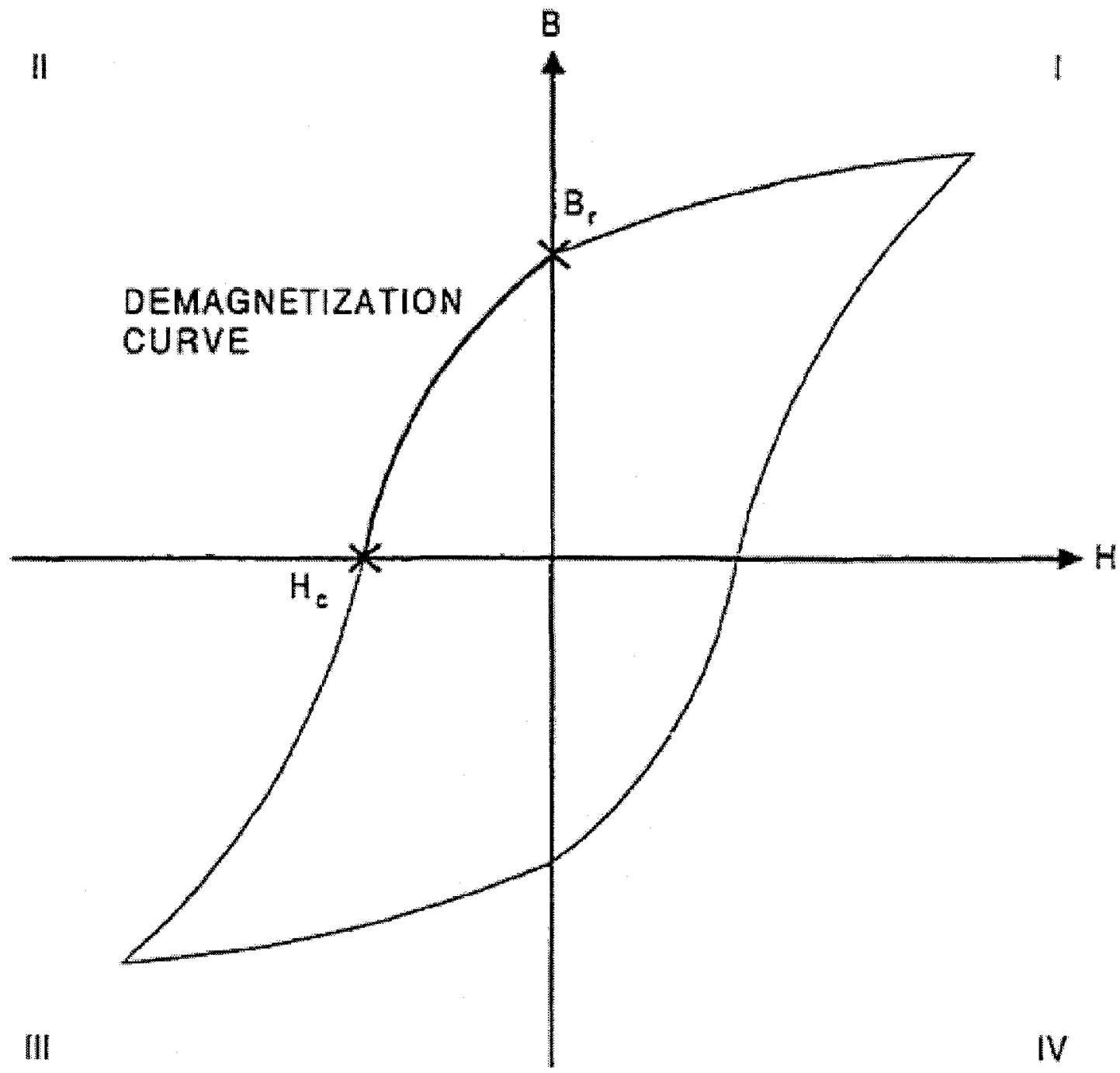

Figure 2.5: Hysteresis Curve Showing Demagnetization Curve, Remanence $\left(B_{r}\right)$ and Coercive Force $\left(H_{C}\right)$, from Wiktowy [30] 
of the magnet. Because little leakage occurs, the majority of the magnetic field is available at the poles of the magnet and it can be therefore readily utilized. Type II magnets, including RECo (Rare-Earth Cobalt) Ceramics, Ferrite Ceramics, and $\mathrm{NbFeB}$ (Neodymium-Iron-Boron), are often made out of ceramic materials, making them brittle and not easily machined. The stability of the type II magnetic material is better than that of the type 1 material with respect to demagnetization fields. However, this stability does not necessarily extend to temperature sensitivity.

Since the late 19th century and earlier, research has been conducted into the influence of changes of temperature on magnetism, as seen by Ashworth [1] in 1903. Generally as operating temperature is increased, the magnetic field intensity or magnetic induction $B$ decreases. This can occur both linearly and non-linearly depending on the magnet material type. The $B$ field decreases until reaching the Maximum Service Temperature, which is the temperature where the magnet can no longer perform its function; or ultimately the Curie Temperature, $T_{c}$, which is the temperature where the material no longer exhibits any magnetic properties. The rate at which the $B$ ficld changes is called the Magnetic Temperature Coefficient.

As can be seen in Table 2.2, there is no definitive trend in temperature characteristics when grouping Type I and II magnets. There are magnet materials whose fields are scnsitive and insensitive to temperature variation found in both Type I and II groups. The highest variation of magnetic field with temperature is found in the ferrite ceramics and NbFeB magnets. The GyroWheel ${ }^{\mathrm{TM}}$ utilizes Type II radially magnetized permanent magnet rings in the rotor made of Crumax ( $\mathrm{NbFeB}$ ). A detailed analysis of these magnets' temperature performance was not carried out within the scope of this thesis as magnetic variations due to temperature are calibrated for when experimentally determining the rate sensing equations. 
Table 2.2: Thermal Characteristics of Various Type I and Type II Permanent Magnets, from Parker [18]

\begin{tabular}{|l|l|l|l|l|}
\hline Magnetic Material & $\begin{array}{l}\text { Alnico VIII } \\
\text { Cast } \\
\text { (Ferrite) }\end{array}$ & $\begin{array}{l}\text { Ceramic 10 } \\
\text { GECo5 } \\
\text { Grade 22/15 }\end{array}$ & $\begin{array}{l}\text { Crumax } \\
3714 \\
(\mathrm{NbFeB})\end{array}$ \\
\hline $\begin{array}{l}\text { Thermal Expansion } \\
\left({ }^{\circ} \mathrm{C} \times 10^{-6}\right)\end{array}$ & 11.0 & 10 & $\begin{array}{l}13 \perp \text { to Ori- } \\
\text { entation } \\
6 \| \text { to Orien- } \\
\text { tation }\end{array}$ & $\begin{array}{l}-0.30 \perp \text { to } \\
\text { Orientation } \\
6.8 \| \text { to Ori- } \\
\text { entation }\end{array}$ \\
\hline $\begin{array}{l}\text { Temperature Coeffi- } \\
\text { cient of B }\left(\% /{ }^{\circ} \mathrm{C}\right)\end{array}$ & -0.02 & -0.20 & -0.045 & -0.110 \\
\hline $\begin{array}{l}\text { Maximum Service } \\
\left.\text { Temperature }{ }^{\circ} \mathrm{C}\right)\end{array}$ & 520 & 400 & 250 & 150 \\
\hline
\end{tabular}

\subsubsection{Magnetic Coils}

Magnetic Coils, or electromagnets, are used to generate magnetic dipole moments for attitude and angular momentum control. Consider a single, plane, wire loop enclosing an area, $A$, through which a current, $I$, is flowing as shown in Figure 2.6. Then the magnetic moment, $m$, is given by:

$$
m=I A n
$$

where $n$ is a unit vector normal to the plane of the loop. The positive sense of the magnetic moment is determined by the right-hand rule; that is, the direction of the magnetic moment is the direction of the thumb of the right hand when the fingers of the right hand are cupped in the direction of the electric current in the loop. For a coil of $N$ turns, the principle of superposition gives:

$$
m=N I A n
$$

The magnetic dipole moment, $d$, depends on the material enclosed by the current carrying coil and is given by:

$$
d=\mu m
$$




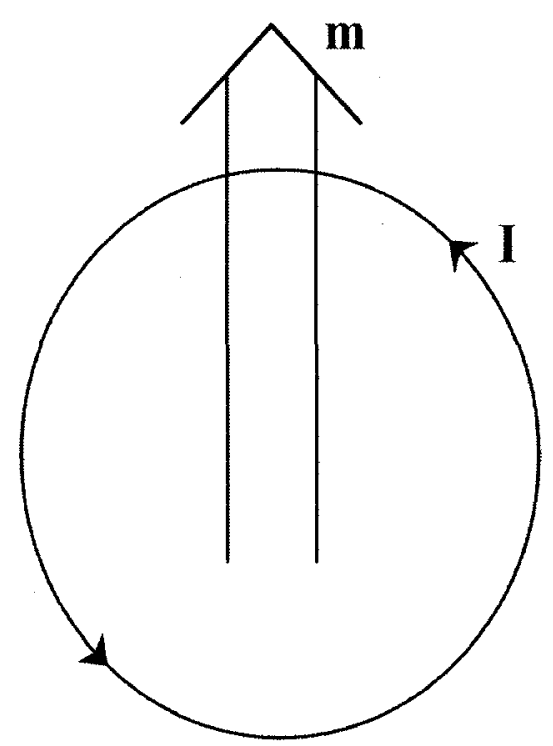

Figure 2.6: Visualization of the Magnetic Dipole Moment Generated by a Wire Loop with Current Flowing Through it.

where $\mu$ is the magnetic permeability of the core material. Thus, for a coil or an electromagnet enclosing a plane area, $\mathrm{A}$, the magnetic dipole moment is given by

$$
d=\mu(N I) A n
$$

From Equation 2.23 it is apparent that to generate a required amount of magnetic dipole moment, parameters such as core material, $\mu$; coil configuration, $N$ and $A$; and the current level, $I$, must be selected appropriately. As discussed by Wertz [29], the most important design parameter is the core material selection. Ferromagnetic materials, such as Permalloy (78\% nickel, $22 \%$ iron), and Permendur (50\% cobalt, $50 \%$ iron) have very high permeabilities that lead to a substantial reduction in power consumption as well as bulk when they are used as the core material. However at rclatively low values of applied magnetic field intensity, the magnetization curves for ferromagnetic materials saturate and exhibit both nonlinearity and hysteresis. Importantly, in ferromagnetic materials, permeability is a function of the magnitude 
of the intensity of the magnetic field. As a result, it is difficult to accurately predict the magnetic dipole moment, a reason why ferromagnetic cores are seldom used and magnetic coils on most satellites have "air" cores.

Accurate prediction and generation of spacecraft magnetic control torques generally requires that the coils be supplied with a constant current as the resistance of the current-carrying element changes with temperature. The constant current is then applied for a certain amount of time to place the spacecraft in the desired attitude. The spccifics of the GyroWhecl ${ }^{\mathrm{TM}}$ electromagnetic torque coils utilized to maintain the desired rotor state are discussed in section 3.1.6, but they do not utilize a constant current system and are thus susceptible to resistance changes due to temperature. They provide varying current which generates torques on the spinning rotor in order to provide 3 axis actuation control torques to the spacecraft. A tilt sensing feedback system ensures the correct current is provided for the desired rotor state and variations due to temperature induced resistance changes are calibrated for when experimentally determining the rate sensing equations.

Interestingly enough, just as the spacecraft thermal control system has a significant impact on the ADCS subsystem design, the spacecraft ADCS subsystem can have a significant influence on the thermal control subsystem design. The attitude of the spacecraft determines the thermal radiation inputs from the Sun and Earth and these inputs affect radiator performance and spaccraft temperatures.

\section{$2.3 \quad$ Gyroscope Theory}

The function of a gyroscope is to measure the orientation of its platform or to sense its angular rate. From Benton [3], the word itself is derived from the Greek gyros meaning "to view" and skopein meaning "rotation". The category of gyroscopes can be subdivided into the two categories of conventional and unconventional gyroscopes and are discussed by Titterton and Weston [25]. Conventional gyroscopes, 
or mechanical gyroscopes, were introduced initially and consist of a rotor, or wheel, spinning at high speeds. Examples include: single axis gyroscopes, flex gyroscopes, dynamically tuned gyroscopes, and the GyroWheel ${ }^{\mathrm{TM}}$ calibrated in this thesis. Unconventional gyroscopes do not rely on the dynamic properties of a rotating body, but still provide measurements of body rotation and are thus called gyroscopes. Unconventional gyroscopes include: vibratory gyroscopes, optical rate sensors, and nuclear magnetic resonance gyroscopes.

\subsubsection{Conventional Gyroscopes}

A conventional gyroscope is a device with a spinning rotor that has been mechanised so that it responds to angular rates. If mounted in a frictionless bearing, the rotor remains fixed in inertial space while the vehicle rotates about it. A torque, applied normal to the angular momentum vector, will have a magnitude equal to the rate of change of the angular momentum. When a torque is applied to a spinning object, the angular momentum vector precesses until it lines up with the applied torque vector.

The angular momentum vector or spin vector can induce another form of motion known as nutation. Nutation is the coning motion of the spin axis about the constant angular momentum axis, when no torque is applied. What results is the angular momentum vector or spin axis traces out a circular path. Whereas precession is the change in direction of the angular momentum axis when a torque is applied.

Some gyroscope designs limit the motion of the spinning wheel by constraining it to move only about one axis to deal with nutation and other complexity problems. These gyroscopes are known as single degree-of-freedom (SDOF) gyroscopes as opposed to two degree-of-freedom (TDOF) gyroscopes or free gyroscopes which allow motion about two orthogonal axes. Almost all conventional gyroscopes fall into one of these two broad categories. 


\section{Single Degree-of-Freedom Gyroscopes}

Initial attempts at designing TDOF gyroscopes were complex and there were crosscoupling effects that did not provide the required results. This resulted in the development of high accuracy gyroscopes sensitive on only one axis, making SDOF gyroscopes the most common type of gyroscope. In broad terms, there are two types of SDOF gyroscopes: the rate gyroscope which is an open loop sensor in nature, and the Rate Integrating gyroscope which provides higher accuracy and is a closed loop sensor in nature.

The rate gyroscope provides an output of the angular rate sensed about the input axis. The gyroscope case has a gimbal attached to it and a torsion bar is utilized to restrain the motion about the defined axis. Fluid, most often a fluorocarbon, in the interior of the casing is utilized to damp the motion of the gimbal and rotor. A pickoff provides an electrical signal whose magnitude is proportional to the gimbal angle.

The Rate Integrating gyroscope is of similar construction to the rate gyroscope, except that the torsion bar is replaced with a ball bearing, and a torquer is added to give a closed loop control. The gyroscope output is derived from the current in the torquer, generated by a servo amplifier. Broxmeyer [4] and Lawrence [15] provide excellent discussions of the SDOF gyroscope and a good example of the use of SDOF gyroscopes is the Hubble Space Telescope as discussed by Dougherty [10].

\section{Two Degree-of-Freedom Gyroscopes}

TDOF gyroscopes, or free gyros, are a more elegant and complete use of the gyroscopic properties of a spinning wheel, since they make use of all available information as opposed to constraining the movement about one axis. As a complete INS requires rotations to be sensed about three independent axes, an INS requires only 
two TDOF gyroscopes instead of three SDOF gyroscopes ${ }^{1}$. This usually results in a lower cost and provides one redundant axis.

The use of a gimbaling system, where a spinning wheel is allowed to rotate within two gimbals, is the most obvious method of designing a TDOF gyroscope. As the spinning wheel is housed within the confines of the gimbals, the wheel is usually relatively small giving the gyroscope a small angular momentum, which in turn generally limits the maximum sensitivity of the instrument. The most common type of TDOF gyroscope is the Dynamically Tuned Gyroscope (DTG), which is also known as the Tuned Rotor Gyroscope (TRG) or Dry Tuned Gyroscope. Given the similarity of the GyroWheel ${ }^{\mathrm{TM}}$ mechanical system with that of a DTG, an appreciation of DTGs is important, and their design and operation will be further discussed.

\subsubsection{Dynamically Tuned Gyroscopes}

The Dynamically Tuned Gyroscope grew out of experiments with spinning wheels supported by joints consisting of two gimbals supported on ball bearings. The gimbal assembly functions as a Hooke's Joint, more commonly known as a universal joint, which allows rotary movement through an angled joint and facilitates the transmission of rotary motion from the spin motor to the rotor or spinning wheel when the input and output spin axes are not parallel. In other words, it allows the spinning wheel or rotor to tilt about both the $\mathrm{x}$ and $\mathrm{y}$ axis. The orientation of the rotor is measured with electromagnetic pickoffs. Cain [5], in his Appendix A, provides an excellent discussion of the kinematics of a Hooke's Joint which demonstrates it is possible to relate the gimbal angles and velocities to the measurements from the pickoffs.

\footnotetext{
${ }^{1}$ In certain spacecraft applications the requirement for rate sensing along a third axis can be alleviated based on the scope of the mission and the spacecraft type using earth and sun sensors for earth and sun pointing missions.
} 
The initial configuration first developed in the 1940s resulted in high nutation and precession rates due to the dynamic effects of the gimbal motion and was not useful $^{2}$. In later designs a flexible joint, or flexure, consisting of torsional springs was introduced, replacing the bearings, or 'pins and jewels', of the gimbal assembly producing a gimbal assembly consisting of a gimbal ring and two flexure joints. The axes of the two pairs of flexure pivots are orthogonal and intersect at a common point on the spin axis. The initial problem with a flexure was that it resulted in spring torques which coupled the rotor to the spin shaft, thus making the system an ineffective inertial reference. If these spring torques were eliminated or canceled the rotor would act as a free body as it would be free from external torques. Early attempts at counteracting the spring action of the flexures were done using magnetic torquers, however this solution was unreliable due to hysteresis effects in the magnetic field. A solution found was to use the dynamic inertia effects of the gyroscope itself, effectively creating equal virtual negative spring rates, to negate the positive spring torques resulting from the flexible joint. A gyroscope incorporating this solution is known as the Dynamically Tuned Gyroscope.

A schematic representation of a DTG, which includes all of the major components common to a DTG is shown in Figure 2.7 from Ower [17]. The components are housed within a casing that serves both to protect the inner workings of the gyroscope and to provide a reference surface so the instrument can be accurately mounted. The DTG ease is sealed, the air evacuated and then refilled with hydrogen gas at a pressure of $1 / 3 \mathrm{~atm}$. This is done to limit the development of air currents. The case also plays an important role in the heat transfer characteristics of the gyroscope.

The motor, usually a hysteresis, three phase type, is mounted rigidly to the case and powers the shaft which is attached to the rotor through the gimbal assembly. The electromagnetic pickoffs, mounted orthogonally, sense the motion of the rotor.

\footnotetext{
${ }^{2}$ These resulting high rates are often considered an unstable configuration.
} 


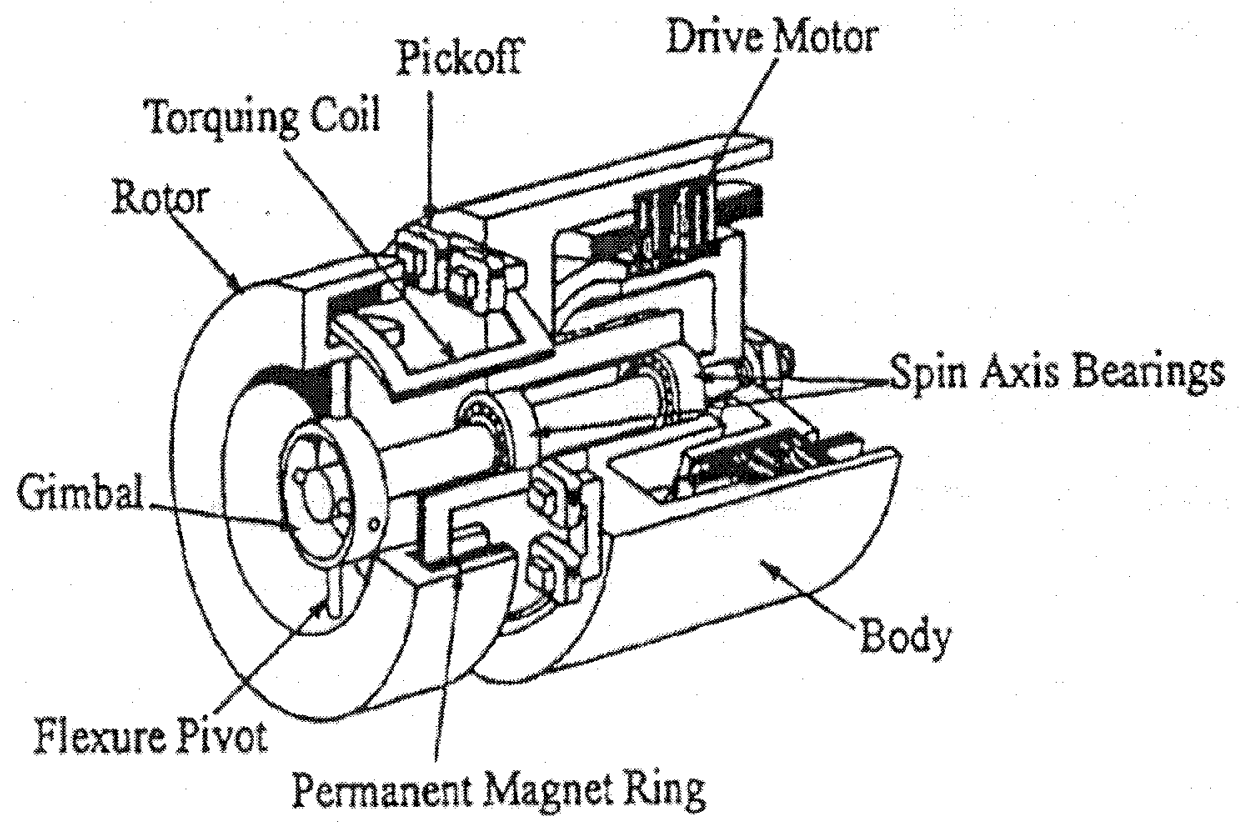

Figure 2.7: Schematic Diagram of a Dynamically Tuned Gyroscope, from Ower [17]

In a closed loop configuration, the output, from these pickoffs is amplified and returned to torquer coils which rebalance, or null the rotor with respect to the shaft. This closed loop configuration is commonly used in strapdown systems where the gyro is attached directly to the vehicle. In this situation, the orientation of the spacecraft is obtained by applying a scale factor to the electric current used in the torquer coils, as the torques required to maintain the rotor at the null orientation are a measure of the external rates of the body on which the sensor is mounted. DTGs are also used in an open loop configuration where the gyro is mounted on a stabilized platform. When this is done the orientation of the vehicle is determined from the pickoff output. The Canadian Strapdown Gyroscope, mod 2, (CSG-2) as discussed and tested by Hartl [12] is an excellent example of a DTG. 


\section{Tuned Condition for Dynamically Tuned Gyroscopes}

As stated earlier the DTG becomes dynamically tuned when the positive spring rates, resulting from the flexible joints, and the virtual negative spring rates, resulting from the dynamic inertia effects of the gyroscope, are designed to be of equal value. In this 'tuned condition' the rotor behaves as a free spinning body and remains inertially fixed regardless of the motion of its case.

To illustrate the concept of the 'tuned condition', the motion of the gimbal during the operation of a tuned-rotor gyroscope is shown in Figure 2.8 from Ower [17]. As discussed by Ower [17], the rotor spin axis is held at a non-zero tilt with respect to the input spin axis, thus at the start of an input spin axis rotation, the inner flexure pivot twists while the outer flexure pivot remains stable. After a quarter rotation of the input spin axis, the inner flexure pivot becomes stable and the outer flexure pivot twists. Over the course of one input spin axis rotation, the inner and outer flexure pivots will both complete two cycles of twisting and relaxing to a stable position. Their torsional displacement will be sinusoidal with respect to time and the frequency of oseillation will be twice the spin frequency. The inner flexure pivot twisting is ninety degrees out of phase with the outer flexure pivot twisting. The two displacement profiles can be considered to be related in quadrature.

The oscillations about the inner and outer gimbal produce a fluttering motion of the gimbal ring. It is this fluttering motion that can be utilized to dynamically tune the gyroscope by negating the positive spring rate of the flexure pivots. The fluttering motion combined with the angular momentum of the gimbal about its spin axis produces a gyroscopic precession torque. This precession torque is proportional to the deflection of the rotor spin axis and therefore can be viewed as a negative spring rate. This negative spring rate is a function of the gimbal inertia and the rotational speed of the system. The DTG dynamic equations will be dealt with 


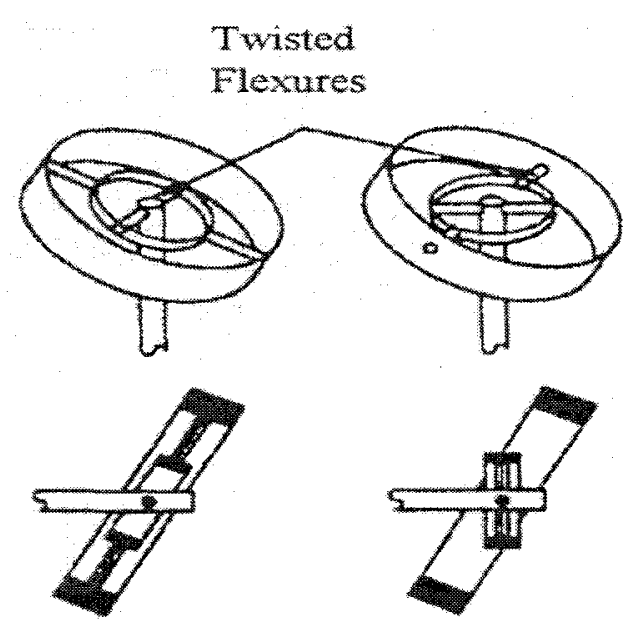

Start of Rotation: Inner Flexures Twisted

A Quarter Turn Later: Outer Flexures Twisted

Figure 2.8: Gimbal Motion in a Dynamically Tuned Gyroscope, from Ower [17]

further in Section 4.1, but the tuning condition is expressed by Craig [6] as

$$
\omega_{s}=\sqrt{\frac{K_{x}+K_{y}}{I_{g x}+I_{g y}-I_{g z}}}
$$

where $K_{x}$ and $K_{y}$ are the $\mathrm{x}$ and y flexure stiffnesses, and the $I_{g x}, I_{g y}$ and $I_{g z}$ terms are the gimbal inertias along their respective axes. A flexure suspended rotor running at a speed $\omega_{s}$ results in the rotor behaving as a free body.

From the tuning condition expressed in Equation 2.24, it is evident that the design of the flexure heavily impacts the tuned speed of the gyroscope. Because the angular momentum of the rotor is directly proportional to the speed of rotation $(H=I \omega)$, it is desirable to have as high a tuned speed as possible, meaning that stiff flexures are desirable. However, a high tuned speed will result in increased bearing wear which reduces the expected life span of the gyroscope. This factor drives the design into having a lower tuned speed with a high rotor inertia to obtain good performance and the highest angular momentum possible. The size of the inertia term is constrained by the overall case dimensions. These are trade-offs which must 
be defined, weighed and evaluated at the time of gyroscope design.

\subsection{Momentum Management}

Now that the theory for the attitude determination portion of the Attitude Determination and Control System has been briefly discussed, the attitude control portion will now be briefly discussed. The overall purpose of the attitude control of spacecraft is to apply torques to re-orient and maintain a desired satellite pointing direction. This involves adding stability against disturbance torques, absorbing cyclic torques, and transferring momentum to the satellite body for the execution of slewing maneuvers.

As discussed by Staley [22], the most common method of spacecraft attitude control is through the direct control of angular momentum. This is achieved by maintaining the spacecraft's momentum in a non-zero state through one of two means: spinning the spacecraft body (spin-stabilized) or including spinning bodies within the spacecraft (three-axis or body stabilized). Spin-stabilized spacecraft will not be discussed as the GyroWheel ${ }^{\mathrm{TM}}$ system does not fall under this category and in general body stabilized spacecraft can be manoeuvred more easily and can achieve higher pointing accuracies. However, as discussed by Wertz and Larson [28], such control systems are generally more complex, more expensive and have more failure modes. In general, for continuous attitude control, some form of internal spinning wheel(s) are utilized with thrusters and/or magnetic torquers applied when the wheels become saturated and are operating at their maximum (or minimum) speed, in order to 'dump' momentum and restore the wheels to their nominal operating speed.

Generally three types of spacecraft devices make use of angular momentum for the purpose of controlling the spacecraft attitude. These include:

- Reaction Wheel - a flywheel with its axis fixed to the vehiclo and designed 
to operate around a zero biased angular momentum. They can spin in either direction, and provide one axis of control for each wheel.

- Momentum Wheel - similar to a reaction wheel except that it is designed to operate at a nominal spin rate providing a biased, or non-zero angular momentum, about its axis of rotation which is usually fixed in the vehicle. This nearly constant angular momentum about the axis of rotation provides gyroscopic stiffness to the other two axes. Varying the speed of the momentum wheel causes torques on the spacecraft about the momentum wheel's axis of rotation allowing precise pointing about this axis and also providing a variable angular momentum storage capability about this axis ${ }^{3}$.

- Control Moment Gyro ${ }^{4}$ - a single or double gimbaled wheel spinning at a constant rate. Control of the direction of the momentum vector in the spacecraft body is provided through the gimbal rings.

A Control Moment Gyro's (CMG) angular momentum is due to the rotor which is spinning about the spin axis with a constant angular rate. With the gimbaled spin axis, a commanded gimbal rotation changes the direction of the angular momentum vector creating a control torque that is parallel to the output axis. The magnitude of this torque depends upon the gimbal rotation rate in addition to the rotor speed. Generally CMGs can produce high-output torques. Additionally, varying the speed of the rotor provides a torque about the third axis. As discussed by Wertz and Larson [28], control systems with CMGs can produce large torques about all three of the spacecraft axes and as a result they are often used for agile or high-rate manoeuvres. However, they require a complex control law and momentum exchange for desaturation. They are also costly to manufacture and heavy.

\footnotetext{
${ }^{3}$ Momentum Wheels have an advantage in their length of life over reaction wheels as they never have to go through a zero speed situation resulting in less wear on the bearings.

${ }^{4}$ Also known as gyrotorquers from Wertz [29] or gimbaled momentum wheels from Ower [17].
} 
As discussed by Ower [17], for high accuracy three-axis attitude control involving the control of stored angular momentum, there are two main methods that are generally employed. These include a multiple-reaction wheel configuration; and a double-gimbaled CMG, both of which are discussed below.

- Multiple-reaction Wheel Configurations have three reaction wheels mounted within the spacecraft in an orthogonal triad. The total angular momentum magnitude and direction are controlled through the variation of the spin rate in each wheel. To provide redundancy, an additional wheel must be supplied for each axis, adding both cost and weight. A more simple configuration can be employed by mounting four wheels in a skewed configuration. In the event of a single wheel failure, the remaining three wheels can be used without degraded performance. However, this multi-reaction wheel configuration can be heavy, is complex and is not practical for small spacecraft given the necessary main and redundant wheels, each driven by an individual electronics box. Additionally the wheels generally have to transition through zero wheel speed situations which can increase design costs due to bearing wear considerations.

- Double-Gimbaled Control Moment Gyros consist of a rotor that is mounted on a two-axis gimbal system. This can replace a complement of three orthogonal mounted reaction wheels. The control of the gimbal angles of rotation provides torques in the two axes perpendicular to the shaft, while varying the speed of the rotor provides a torque about the third axis. This provides the CMG with the capability of steering the angular momentum vector along all three axes. Two of these Double-Gimbaled CMGs creates a fully redundant system. Figure 2.9 from Bender and Brehove [2] shows a very simple double-gimbaled CMG or momentum wheel. The rotor is mounted on a table and the two axes of tilt are controlled with three linear jackscrews. The jackscrews are actuated with stepper motors to produce a \pm 6 degree rotation about the two 
axes orthogonal to the rotor spin axis.

As discussed by Ower [17], although the double-gimbaled CMG approach appears to be superior to the multiple-wheel concept, the jackscrew actuators operate in discrete steps, that limit the overall pointing accuracy and result in less nutation control. The double-gimbaled CMG is complex, employing a high number of mechanisms which results in increased mass and cost and most importantly lower reliability. As a result, both approaches are ill suited for employment in small spacecraft.

A system that provides the same function as the double-gimbaled CMG, and therefore realizes the mass and cost savings of multiple wheels, without the disadvantages detailed above of double-gimbaled CMG mass, cost, and complexity would be advantageous, especially in the ficld of small satellite ADCS. The GyroWheel ${ }^{\mathrm{TM}}$ is such a system. 

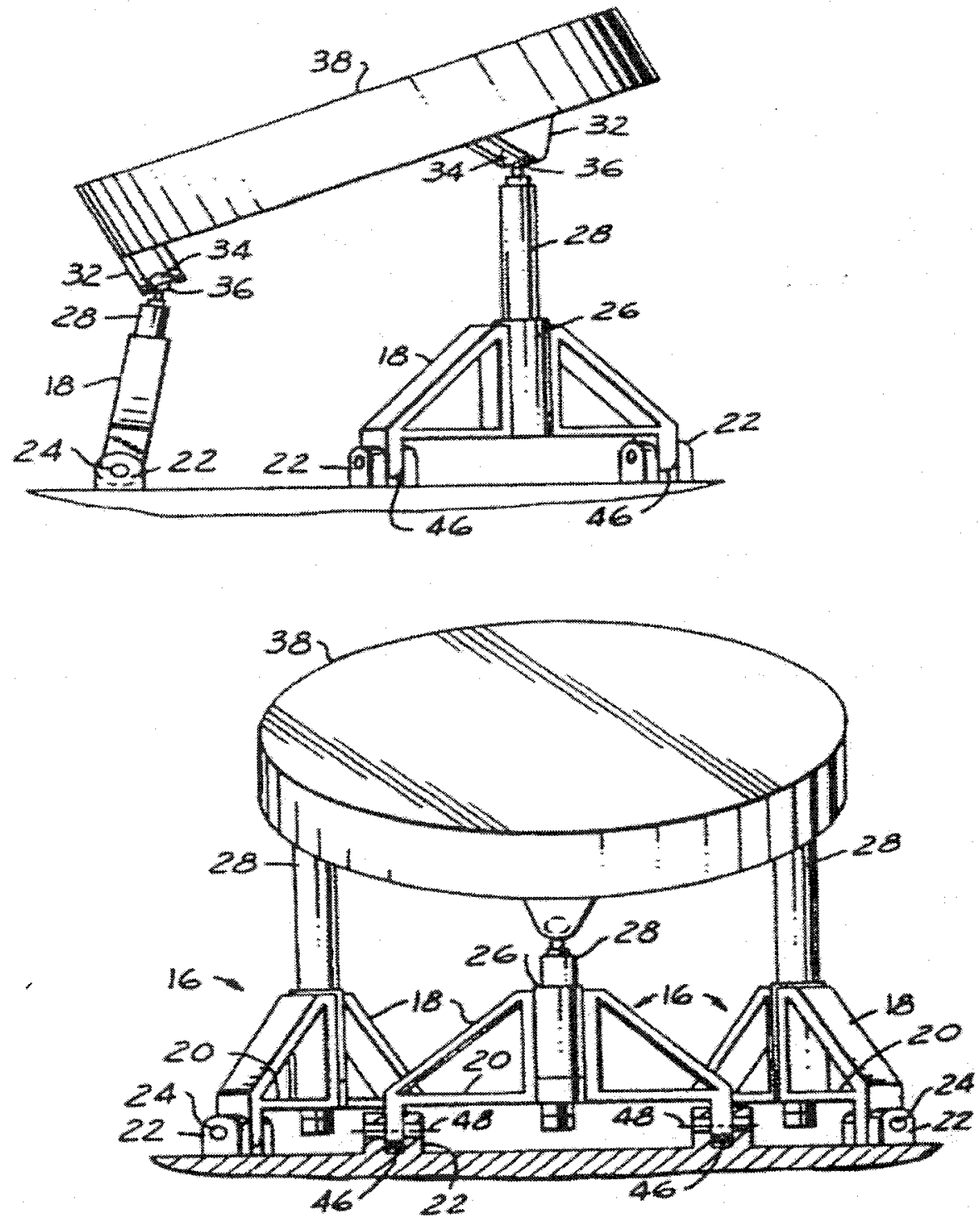

Figure 2.9: Double-Gimbaled Momentum Wheel, from Bender and Brehove [2] 


\section{Chapter 3}

\section{The GyroWheel ${ }^{\mathrm{TM}}$ System}

The GyroWheel ${ }^{\mathrm{TM}}$ is a form of double gimbaled CMG, but based on using a spinning flex-gimbal system as opposed to the usual non-spinning motor driven gimbals that are typically used in CMG torque actuators. Although it can provide control torques about all 3 axes, the device is about the same size and power as a standard single axis momentum wheel of the same momentum class. This allows for significant reductions in the size, mass and power required, while still maintaining the same three axis momentum steering capability of a CMG. Of course, one difference between a CMG and the GyroWheel ${ }^{\mathrm{TM}}$ is that usually the CMG has a much larger tilt range. However, for most spacecraft applications which are generally pointed at a single target like the Earth or the Sun, the 7-degree articulation range of the GyroWheel ${ }^{T M}$ is more than adequate.

Additionally since the GyroWheel ${ }^{\mathrm{TM}}$ design is based on the structure of a DTG, with the exceptions of a significantly larger rotor and tilt angle sensing, the device functions as a precision two axis rate sensing gyroscope at the same time as providing spacecraft control torques. The torsional stiffness provided by the cross flexure pivots of the spinning flex-gimbal system enables the GyroWheel ${ }^{\mathrm{TM}}$ to be dynamically tuned. 


\subsection{System Components}

An exploded view of the GyroWheel ${ }^{\mathrm{TM}}$ is shown in Figure 3.1, with a cut-away isometric view shown in Figure 3.2. A cross-sectional view is shown in Figure 3.3. The system consists of six main components:

- Spin Motor

- Bearing Cartridge Subassembly

- Gimbal Assembly

- Rotor

- Tilt Sensors

- Torque Coils

- Drive and Control Electronics

Detailed descriptions of the six components, and their unique characteristics, are provided below.

\subsubsection{Spin Motor}

An 8-pole brushless DC motor is used to apply the spin torque to the shaft. It mounts to the bottom end of the shaft as shown in Figures 3.1 and 3.3. The motor uses integral hall effect sensors for commutation. All moving components are contained in a housing and situated above the drive and control electronics boards.

\subsubsection{Bearing Cartridge Subassembly}

As discussed by Tyc [26], one of the critical elements of any spinning mechanism for spacecraft applications is the bearing. Considerable attention has been given to the 


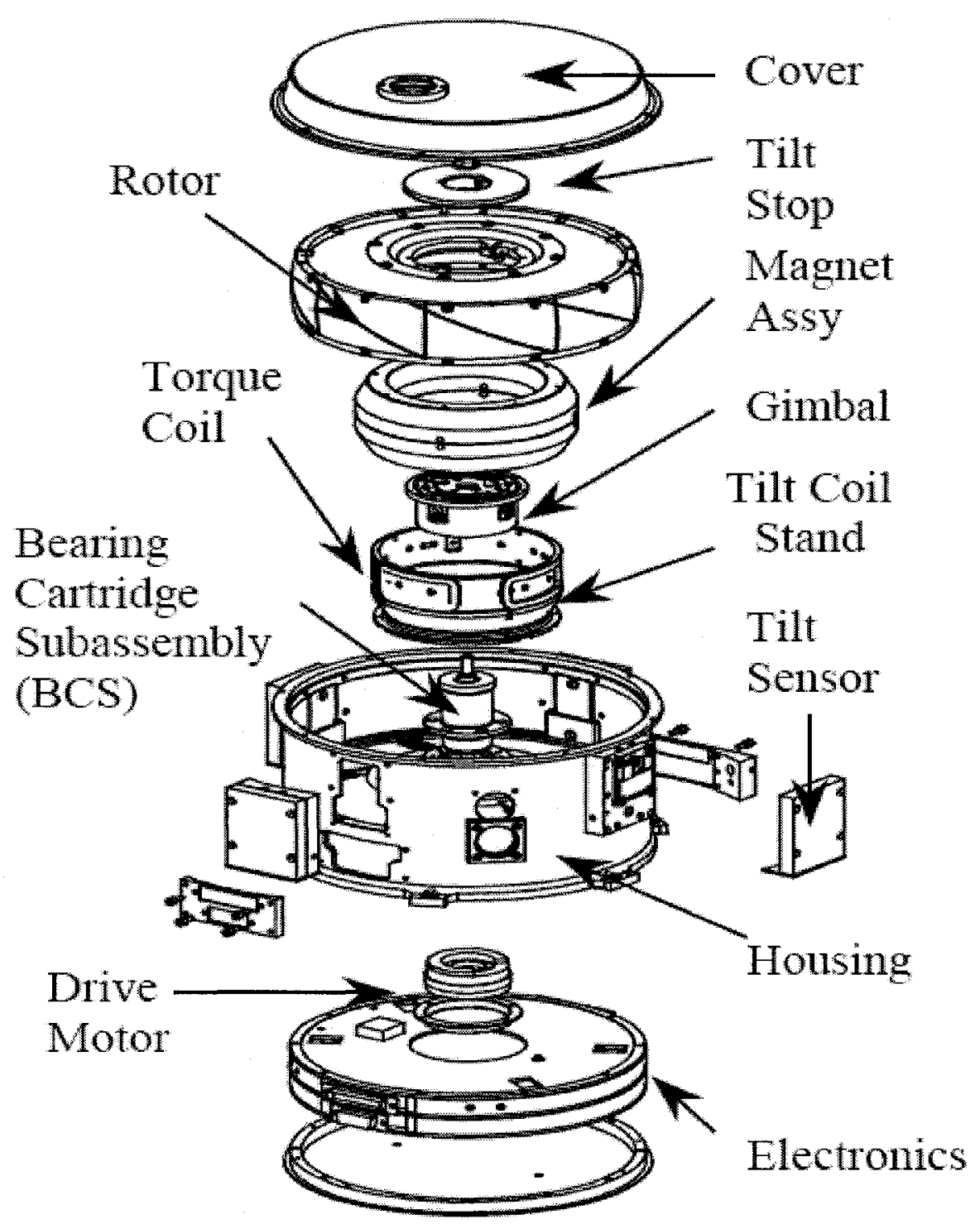

Figure 3.1: GyroWheel ${ }^{\mathrm{TM}}$ Exploded View 


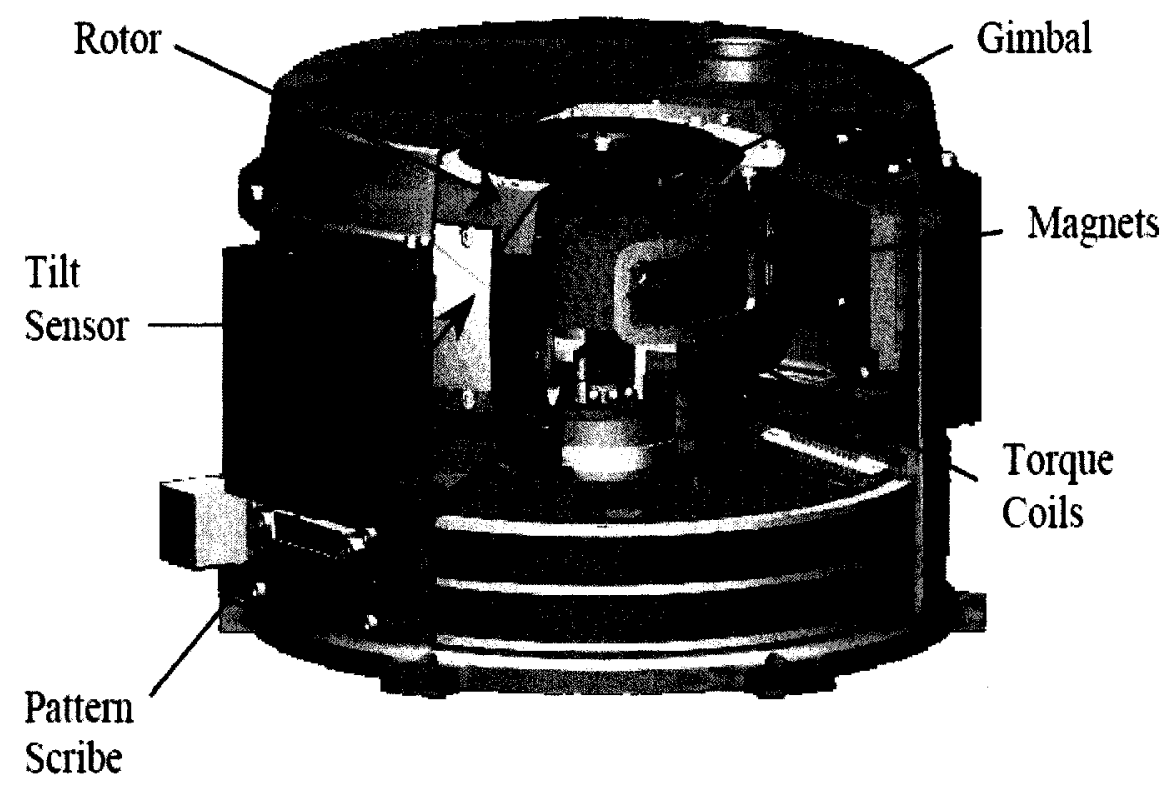

Figure 3.2: GyroWheel ${ }^{\mathrm{TM}}$ Cut-away View

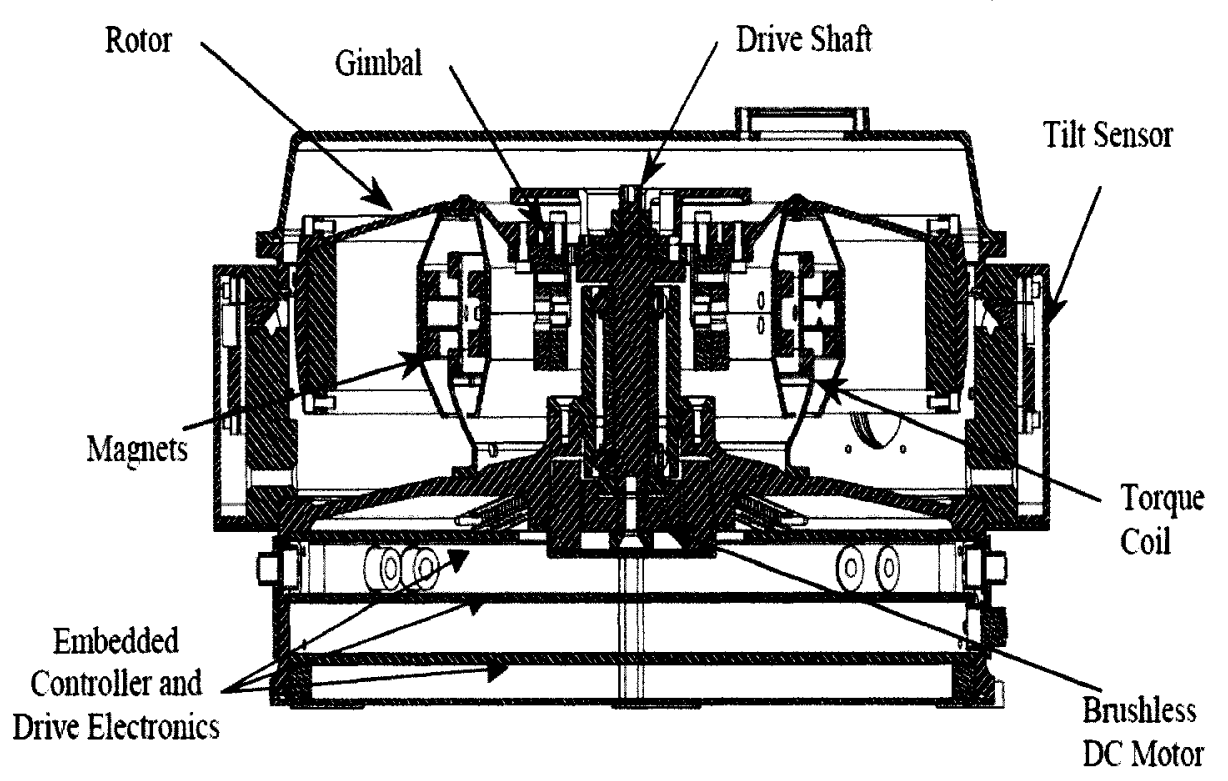

Figure 3.3: GyroWheel ${ }^{\mathrm{TM}}$ Cross-Sectional View 
bearing design for the GyroWheel ${ }^{\mathrm{TM}}$ to ensure a reliable, long life bearing design that will exceed 15 years. The spacecraft industry tends to be extremely conservative regarding bearing mechanisms as they have been the cause of many premature mechanism failures in the past. To effectively deal with these issues, the drive shaft and the bearings have been combined into a single Bearing Cartridge Subassembly (BCS). The integrated BCS allows for the entire subassembly to be manufactured, assembled, and tested by a single bearing supplier allowing for better quality control. Additionally, an integrated BCS permits very high shaft alignment tolerances to be maintained. Unlike the BCS superduplex pair bearing, most bearings are assembled into the wheel separately (top and bottom). This leads to misalignments, pre-load variations and other quality control deficiencies. The GyroWheel ${ }^{\mathrm{TM}}$ bearing cartridge allows for complete inspection, testing and acceptance of the bearing prior to being assembled into the wheel. This is important for achieving high rate sensing accuracy. The BCS was sealed in a hermetic container at the bearing suppliers facilities and shipped for GyroWheel ${ }^{\mathrm{TM}}$ integration as a "drop in" unit to the flight hardware. This step was undertaken in a class 100 clean room facility to ensure no contamination of the BCS unit during final integration of the device. Once assembled, the mechanism enclosure (top part of housing) is sealed to maintain the internal cleanliness level.

\subsubsection{Gimbal Assembly}

The drive shaft of the spin motor is coupled to the spinning rotor by the flexgimbal suspension system. The gimbal assembly consists of a hollow cylindrical section, or gimbal ring, with two pairs of crossed-flexure pivots. Kinematically, the gimbal behaves exactly like a U-joint (also called a Hookes joint) which has two pivoting axes and a "fluttcring mass" (sometimes referred to as the "cross-piece" in a U-joint) that connects these two pivots. However, rather than using bearings or torsion bars at the two orthogonal axes, the gimbal uses the integral two pairs of cross-flexure pivots 


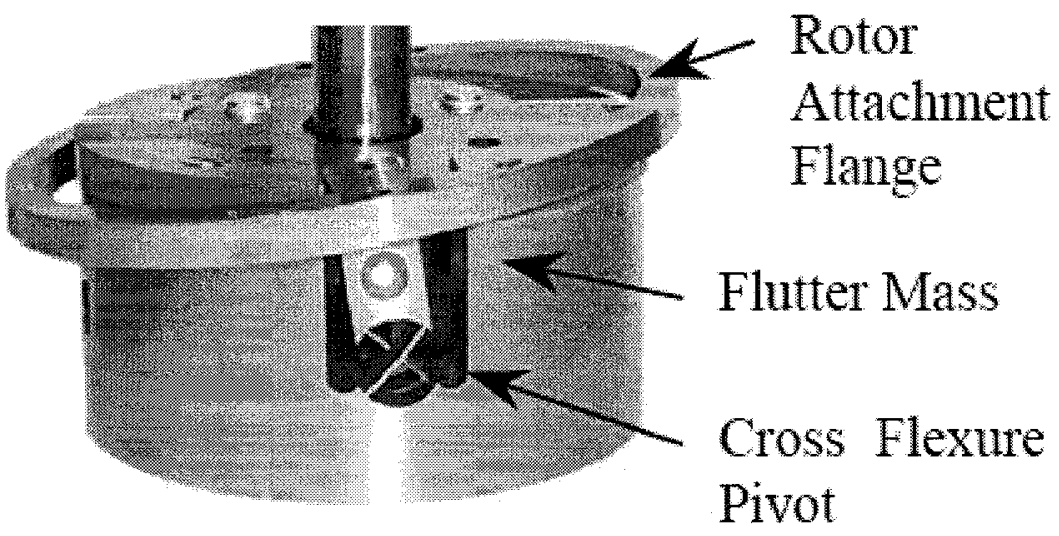

Figure 3.4: GyroWheel ${ }^{\mathrm{TM}}$ Gimbal

that enable tilting the spinning rotor up to 7 degrees from the shaft or null position. The GyroWheel ${ }^{\mathrm{TM}}$ gimbal and crossed-flexure pivots are shown in Figure 3.4.

The axes of rotation of the two pairs of pivots are orthogonal to each other and the drive shaft. The drive shaft is coupled to the inner gimbal ring through a web structure and the outer gimbal is coupled to the rotor via the rotor-mounting flange. The cross-flexure pivot consists of two thin beam-like strips, which cross each other without touching. The ends of the strips are connected to the rigid bodies, meaning the shaft and gimbal, or the rotor and gimbal. The result is a joint which allows rotational motion about one axis while remaining stiff about the other two. As shown by Cain [5], the crossed-flexure pivots also keep translational motion about all three axes to a minimum. As the body attached to the top face rotates relative the bottom face, each of the two flexures will bend such that the intersection point of the two flexures is maintained at the center of rotation.

Use of the crossed-flexure pivots at the hinge points allows for infinite life of the gimbal, as the opcrating stresses seen by the flexures are designed to be considerably lower than the "fatigue limit" of the very high strength steel used for the gimbal flexures. This means that the material used will not wear out or experience any 
fatigue. As stated by Ower [17], "the gimbal assembly has been designed to accept deflections of 10 degrees about each crossed-flexure pivot axis. This is far larger than the typical maximum deflection of 0.1 degrees found in dynamically tuned gyros", discussed above.

When the rotor is tilted and is spinning, the gimbal ring, which is the piece that connects one set of hinge points with the other orthogonal set of hinge points, tends to flutter at twice the spin frequency and is also referred to as the "flutter mass" (exactly like the cross piece in a conventional U-joint discussed above). The stiffness of the cross-flexure pivots impart a small restoring torque to the rotor as it is tilted away from the null position. This allows for "tuning" of the system. The magnitude of the flexure stiffness, incrtia or size of the flutter mass (i.c., gimbal ring or cross-piece), and the rotor spin speed all have a direct bearing on the "tuning" condition. The dynamics of this flex-gimbal is such that the fluttering mass tends to create what is referred to as a "dynamic stiffness" of the flexures. At a particular spin speed the inertial forces due to the fluttering gimbal ring tend to cancel the spring forces of the flexure and the dynamic stiffness becomes zero. At this "tuned" speed, the rotor behaves (to a first order approximation) very ncarly like a freely spinning rotor in space (i.e., as if it had no suspension system). ${ }^{1}$ The rotor will nominally behave as a freely floating rotor when:

$$
K-J \omega_{s}^{2}=0, \text { and } J=\frac{1}{2}\left(I_{g x}+I_{g y}-I_{g z}\right)
$$

where $K$ is the flexure stiffness, $\omega_{s}$ is the tuned speed and $I_{g x}, I_{g y}, I_{g z}$ are gimbal inertias.

It is this feature that allows the device to function as a precision gyroscope while at the same time acting as a 3-axis torque actuator. This "tuned" speed can be set to any value in the bias speed range of the device (1500 to $5200 \mathrm{rpm}$ ) by simply changing the size of the flutter mass in the gimbal, thus changing the gimbal inertias.

\footnotetext{
${ }^{1}$ If tuning was not possible then high precession rates would result, a situation which, in the past, has led to the belief that the predecessors of the DTG were unstable.
} 


\subsubsection{Rotor}

The primary component of the GyroWheel ${ }^{\mathrm{TM}}$ is a stainless steel rotor with a spin inertia of $0.0197 \mathrm{~kg} \mathrm{~m}^{2}$. The rotor is mechanically suspended from the drive shaft through the innovative flexgimbal system that allows the rotor to be actively tilted while spinning. This tilting action provides torques in the two axes perpendicular to the shaft, while varying the speed of the rotor provides a torque about the third axis.

The rotor includes a magnet assembly with four radially magnetized permanent magnet rings. Near the outer diameter of the rotor is an annular cavity where two of the permanent magnet rings are mounted on the inner walls, and two are mounted on the outer walls of the annular cavity. The magnet assembly is used to impart torques to the rotor using electromagnetic coils (or torque coils) which are positioned between the inner and outer permanent magnet rings.

To accommodate the 7 -degree spin axis tilt required to provide the momentum steering, the outer surface of the rotor has the shape of a spherical section. This ensures that the gap between the rotor surface and the tilt sensors is constant, a requirement driven by the tilt sensor design. A triangular pattern, consisting of 8 exactly matching sawtooth patterns, has been machined on the outside spherical surface of the rotor and is used for sensing tilt angle and spin rate as discussed below.

\subsubsection{Tilt Sensors}

As discussed by Ower [17], a unique tilt sensor design was required on the system for two reasons. First, unlike a DTG, tilt cannot be determined by measuring the gap between the bottom of the rotor and the stationary case. This is a result of the comparatively large tilt angles. Second, in order to obtain a good external ratesensing estimate, the rotor spin rate must be controlled rather than the motor spin 
rate, meaning that a resolver or other tachometer-like device on the input motor shaft is not as suitable as a spin rate sensor. Additionally, when the spin axis is tilted with respect to the null position, the rotor spin rate can still be measured utilizing this tilt sensor design.

The instantaneous tilt state of the rotor in two axes and the rotor angular velocity is sensed by two pairs of directly opposing optical tilt sensors. Each of the four sensors are located at 90 degree intervals around the GyroWheel ${ }^{\mathrm{TM}}$ housing and sense the edge transitions of a triangular sawtooth pattern machined on the outside spherical surface of the rotor (shown in Figure 3.1). The sawtooth pattern has different optical properties than the rest of the rotor, and the timing between consecutive pulses generated by the sensed edge transitions can then be calibrated to provide a precise measure of the velocity, as well as the tilt angle about an axis orthogonal to the line joining two opposing tilt sensors and the spin axis.

The specific tilt sensor measurement system is shown schematically in Figure 3.5, from Schultz [19]. In Figure 3.5(b) the general angle measured by the $\mathrm{i}^{\text {th }}$ sensor as stated by Schultz [19], is:

$$
\phi_{i}=\left[\frac{V D}{V V}-0.5\right] 20^{\circ}
$$

where $\phi_{i}$ represents either of the sensor tilt angles $\phi_{x}$ and $\phi_{y}$ which are the tilts around the $\mathrm{x}$ and $\mathrm{y}$ case axes, respectively. Spin rate is determined by taking the time between consecutive leading edges of the sensor pattern (VV in Figure 3.5) based on the tilt of the rotor.

As discussed by Ower [17] the tilt and spin rate measurements have a discrete output that are unlike traditional tilt and spin rate measurements. Specifically, the update frequency of the tilt and spin measurement depends on both the spin rate and the number of saw tooth patterns machined on the outer surface of the rotor. For this application with a nominal speed of $1500 \mathrm{rpm}$, or $25 \mathrm{rps}$, the update frequency is $200 \mathrm{~Hz}$ as there are a total of 8 sawtooth patterns machined on the rotor. 


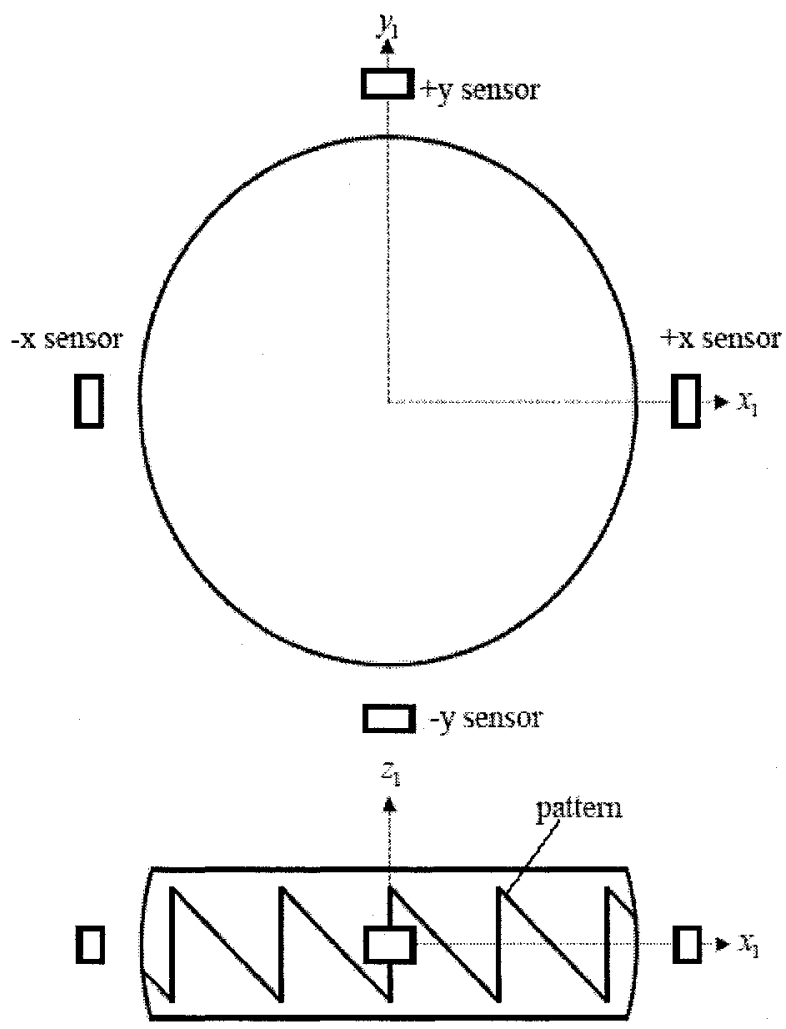

(a)

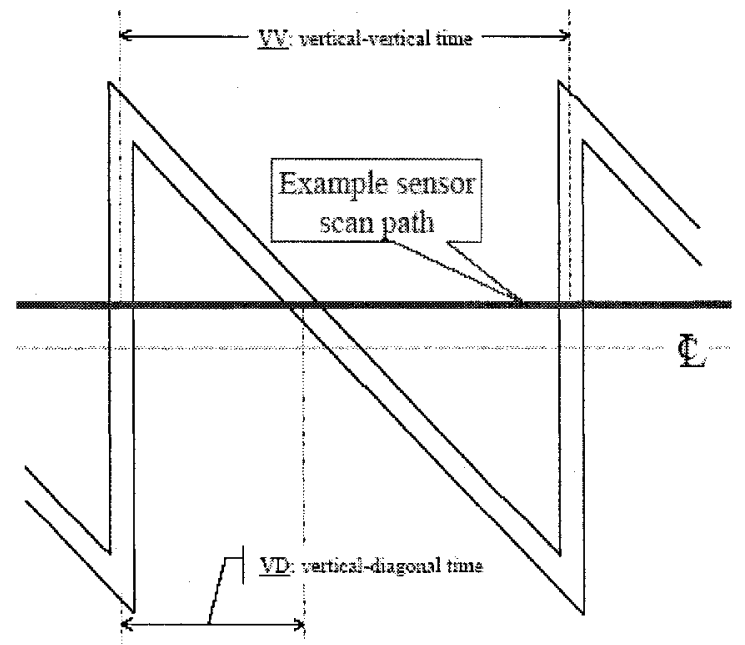

(b)

Figure 3.5: Schematic Representation of GyroWheel ${ }^{\mathrm{TM}}$ Tilt Sensor Measurement, from Schultz [19]. (a) Tilt sensor arrangement around the rotor, (b) Tilt sensor pattern showing light scan path 


\subsubsection{Torque Coils}

Electromagnetic coils or torque coils acting on the permanent magnets within the rotor are used to maintain the desired rotor state. The permanent magnets in the rotor generate a radial magnetic field. The electromagnetic torque coils, which are air core conductive coils, are placed into the radial magnctic field with the coil normal direction aligned with the radial magnetic field. When a known current is passed through the coil it interacts with the magnetic fields of the rotor permanent magnetic rings, creating a Lorentz force, which generates a control torque on the rotor. The torque coils are mounted to a stand that is attached to the GyroWheel ${ }^{\mathrm{TM}}$ stationary housing and are arrayed at 90 degree intervals. They protrude into the rotor cavity and are positioned in the gap between the inner and outer permanent magnet rings as shown in Figure 3.3. The magnet assembly and the torque coils together form four wide-gap, non-contact magnetic actuators that impart forces to the rotor.

By applying a voltage across the torque coils, the resulting current creates a magnetic dipole moment. The radially magnetized permanent magnet rings interact with the magnetic dipole moment to create an axial (parallel to the spin axis) force on the rotor near its outer edge. By applying the opposite signed voltage on the torque coil positioned on the opposite side of the rotor, a torque can be applied on the rotor about the axis orthogonal to the line joining the other two opposed torque coils and the spin axis (vice versa for the other tilt axis). As the forces applied on opposite sides of the rotor are equal and have opposite sign, there is no net axial force acting on the rotor. Therefore pairing up the coils so that one imparts a positive force on the rotor and the opposite coil imparts a negative force ensures that a pure torque or moment is imparted to the rotor. These magnetically generated torques are used to steer the momentum vector of the spinning rotor.

The torque scale factor relates coil current to the torque generated by the torquer and therefore the magnitude of current used in the torque coils is related to the 
external body rates. The large tilts of the GyroWheel ${ }^{\mathrm{TM}}$ rotor and the changes in temperature experienced by the torque coils and permanent magnet rings cause the torque scale factor not to be constant over the \pm 7 degree tilt and $9^{\circ} \mathrm{C}$ to $55^{\circ} \mathrm{C}$ temperature operating range. These need to be calibrated to ensure accuracy of the external rate sensing capability.

\subsubsection{Drive and Control Electronics}

As discussed by Senez [21], a digital control system based on the radhard TEMIC TSC21020F DSP device maintains the tilt angles and the rotor speed trajectory as commanded by the spacecraft attitude control system. The electronics are contained in the bottom half of the GyroWheel ${ }^{\mathrm{TM}}$ housing as shown in Figure 3.1 and 3.3. The Electronics Processing Board contains all of the digital electronics. Radiation hardened parts are used to allow for long life applications in a large range of orbits. The external interface is an RS- 422 serial interface. ${ }^{2}$

The DSP has significant processing capability; however only 25 percent of the capability will be used for the internal GyroWheel ${ }^{T M}$ processing. The remaining processing capability has the potential to be used for the ADCS processing. The GyroWheel ${ }^{\text {TM }}$ can therefore be used as an ADCS processing node on the bus. This effectively provides the capability of an ADCS processor without an impact on mass or the requirement for additional power.

The Mechanism Interface Board contains all of the analog electronics, including the voltage reference, a custom rad-hard high accuracy DAC, torque coil drivers, and the brushless DC motor driver. The board also includes a rad-hard 16 bit ADC and a 16 channel multiplexer that enables the processing of inputs from various sensors used to provide calibration inputs and temperature readings. Temperature sensors are embedded throughout the housing to provide temperature input for rate

\footnotetext{
${ }^{2}$ An optional 1553 bus interface is also available.
} 
sensing routines. Measurements of the rotor speed and tilt angles, and the torque coil currents are used in a proprietary algorithm that is implemented within the digital controller to establish the spacecraft angular rates about two axes.

The Power Control Module contains the DC-DC converters that provide all the required secondary voltages from the external unregulated 28 Volt supply.

\subsection{Principle of Operation}

\subsubsection{Steerable Momentum Wheel}

As discussed by Ower [17], the nominal rate of spin of the GyroWheel ${ }^{\mathrm{TM}}$ has been chosen to coincide with the tuned speed. The rotor spin rate is controlled over a range of $\pm 15 \%$ of the tuned speed in order to vary the magnitude of the rotor momentum vector. The tilt of the spin axis is varied over a range of \pm 7 degrees about the two orthogonal tilt axes in order to vary the orientation of the momentum vector with respect to the GyroWheel ${ }^{\mathrm{TM}}$ case. Because of this the system will frequently operate in a de-tuned condition and at a non-zero tilt. To avoid spin rate or tilt saturation, the ACS of the spacecraft will be required to 'dump' momentum by generating external torques on the spacecraft. This is achieved by using torque actuators such as thrusters or magnetic torque rods. Coinciding with this action, the GyroWheel ${ }^{T M}$ can be commanded back to its nominal momentum state. The GyroWheel ${ }^{\text {TM }}$ system is superior to other gimbaled wheels because if a torque coil were to fail, the rotor spin axis would cone inwards until the tilt reached the null position, causing the GyroWheel ${ }^{\mathrm{TM}}$ to function like a fixed momentum wheel with zero tilt, still providing gyroscopic stabilization about two axes. 


\subsubsection{External Rate Sensing}

In a TRG, the tilt angle is regulated at zero tilt. The torque required, and therefore the current required on the torque coil, to maintain the rotor at this position is proportional to the angular velocity of the external body. In the GyroWheel ${ }^{T M}$ the torque coils will be used to steer the rotor spin axis as determined by the demands of the spacecraft ACS. Since the GyroWheel ${ }^{\mathrm{TM}}$ is designed to be used as a bias momentum device and it is operated at speeds other than the tuned speed, it has been calculated that with calibration the GyroWheel ${ }^{\mathrm{TM}}$ can be utilized within $\pm 15 \%$ of the tuned speed (or bias speed) to maintain the high rate sensing precision.

The rate sensing modeling and calibration required for the GyroWheel ${ }^{\mathrm{TM}}$ is discussed in detail in Chapter 4, but in general what is required is an accurate characterization of and calibration for the torques required to steer the rotor momentum vector along a time-varying temperature, tilt and spin rate profile. The difference between the measured control torque and the predicted torque, processed through the rate sensing algorithm in the digital controller, is a measure of the external rates of the spacecraft body. Additionally, as discussed by Ower [17], the control bandwidths for the GyroWheel ${ }^{\mathrm{TM}}$ spin and transverse-axes were designed to be two orders of magnitude higher than the rate sensing bandwidth. This bandwidth separation ensures the rate-sensing estimation is solely influenced by the steady state behaviour of the spin and transverse-axes control systems.

\subsubsection{Performance Characteristics}

The performance characteristics of the GyroWheel ${ }^{\mathrm{TM}}$ unit are outlined in Table 3.1. The GyroWheel ${ }^{\mathrm{TM}}$ design is scaleable for a large momentum range of $1-16 \mathrm{~N} \mathrm{~ms}$. As discussed by Senez [21], the bias momentum that is required can be set by varying the bias speed and also by adding mass to the rim of the rotor. The GyroWheel ${ }^{\text {TM }}$ calibrated for this research, utilized on SCISAT-1, was the $4 \mathrm{Nms}$ version with a 
nominal speed of $1500 \mathrm{rpm}$ and speed range of $1275-1725 \mathrm{rpm}$.

Separate testing also confirmed that the power required to provide the control torques about the two tilt axes is extremely low (fractions of a watt), confirming that most of the power requirements of this device are due to the motor and drive electronics which is similar to conventional single axis momentum wheels. For the engineering prototype, with nominal speed of $1500 \mathrm{rpm}$ and bias angular momentum of $1 \mathrm{Nm} \mathrm{s}$, the average power was measured to be $5.7 \mathrm{~W}$ (4.5 W for the GyroWheel, most of which is for the spin motor, and $1.2 \mathrm{~W}$ for the drive electronics). Additionally, from Tyc [27], the testing of the engineering prototype demonstrated that very large peak torques can be obtained about the tilt axes with only a very minor impact on the overall peak power requirements. This may be an advantage for some missions.

\subsubsection{Measured Values}

The 'measured' values provided by the GyroWheel ${ }^{\mathrm{TM}}$ system are as follows:

- $i_{x}$ - is the current in the torque coils physically located on the GyroWheel TM $\mathrm{x}$ case axis

- $i_{y}$ - is the current in the torque coils physically located on the GyroWheel ${ }^{\text {TM }}$ y case axis

NOTE: These definitions of $i_{x}$ and $i_{y}$ are opposite from the conventional rate sensing definitions of the two currents in gyro calibration. The above definitions were adopted based on previously defined conventions for GyroWheel ${ }^{\mathrm{TM}}$ momentum actuation.

- $\phi_{x}$ - is the tilt, in radians, of the rotor around the GyroWheel ${ }^{\mathrm{TM}} \mathrm{x}$ axis, as provided by the tilt sensors physically located on the GyroWheel ${ }^{\mathrm{TM}} \mathrm{y}$ axis. 
Table 3.1: GyroWheel ${ }^{\text {TM }}$ Flight Model Characteristics

\begin{tabular}{|c|c|}
\hline Size & $23.5 \mathrm{~cm}$ dia. $\mathrm{x} 13.5 \mathrm{~cm}$ high \\
\hline Momentum Range & $1-16 \mathrm{Nms}$ \\
\hline Mass & $\begin{array}{c}5.5 \mathrm{~kg} @ 4 \mathrm{Nms} \text { config } \\
6.75 \mathrm{~kg} @ 16 \mathrm{Nms} \text { config }\end{array}$ \\
\hline $\begin{array}{l}\text { Power } \\
\text { Steady State } \\
\text { Maximum } \\
\text { (full torque 3-axes) }\end{array}$ & $\begin{array}{l}15.5 \mathrm{~W} @ 1500 \mathrm{rpm} \\
101 \mathrm{~W} @ 6000 \mathrm{rpm}\end{array}$ \\
\hline $\begin{array}{l}\text { Min. Reaction Torque } \\
\text { Spin axis } \\
\text { Tilt axes }\end{array}$ & $\begin{array}{l}76 \mathrm{mNm}(\text { at } 1200 \mathrm{rpm}) \\
122 \mathrm{mNm}(\text { at } 0 \mathrm{deg} \text { tilt })\end{array}$ \\
\hline Gyro Bias Stability & $<1 \mathrm{deg} / \mathrm{hr}$ \\
\hline Speed Range & $1200-6000 \mathrm{rpm}$ \\
\hline Max Rotor Tilt Angle & $\pm 7 \mathrm{deg}$ \\
\hline On-board Processing Capability & $\begin{array}{l}16 \text { MIPS total } \\
12 \text { MIPS available for ADCS }\end{array}$ \\
\hline Static Balance & $<1 \mathrm{gmcm}$ \\
\hline $\begin{array}{l}\text { Equivalent Dynamic Balance } \\
0 \text { deg tilt } \\
7 \text { deg tilt }\end{array}$ & $\begin{array}{c}0.01 \mathrm{gm} \mathrm{cm}^{2} \\
8 \mathrm{gm} \mathrm{cm}^{2}\end{array}$ \\
\hline Radiation Tolerance & $\begin{array}{c}100 \mathrm{kRad}(\mathrm{Si}) \text { total dose } \\
\text { immune to destructive SEEs }\end{array}$ \\
\hline Operating Life & $>10 \mathrm{yrs}$ \\
\hline Input Voltage & $28 \pm 6 \mathrm{~V}$ \\
\hline Command \& Data I/F & $\begin{array}{l}\text { Serial RS-422 } \\
\text { MIL-STD 1553B (option) }\end{array}$ \\
\hline
\end{tabular}


- $\phi_{y}$ - is the tilt, in radians, of the rotor around the GyroWheel ${ }^{\mathrm{TM}} \mathrm{y}$ axis, as provided by the tilt sensors physically located on the GyroWheel ${ }^{\mathrm{TM}} \mathrm{x}$ axis.

- $\dot{\phi}_{x}$ - is the time derivative of $\phi_{x}$ in radians per second.

- $\dot{\phi}_{y}$ - is the time derivative of $\phi_{y}$ in radians per second.

- $\omega_{z}$ - is the wheel speed around the GyroWheel ${ }^{\mathrm{TM}}+\mathrm{Z}$ axis, or spin axis.

- $T_{h, x}$ - is the Housing temperature $\mathrm{x}$ value in degrees Celsius. This value will be used to calculate the appropriate calibration values based on the temperature of the GyroWheel ${ }^{\mathrm{TM}}$

- $T_{h, y}$ - is the Housing temperature y value in degrees Celsius. This value will be used to calculate the appropriate calibration values based on the temperature of the GyroWheel ${ }^{\mathrm{TM}}$ 


\section{Chapter 4}

\section{Dynamically Tuned Gyroscope Dynamics, Rate Sensing, and Testing Theory}

As described in Chapter 2, DTGs are rather complex in comparison to other gyroscopes. The additional complexities of the GyroWheel ${ }^{\mathrm{TM}}$ System, including a tilting rotor and operations at $\pm 15 \%$ of the tuned speed are described physically in Chapter 3. This Chapter elaborates on the dynamics of both DTGs and the GyroWheel ${ }^{T M}$ and mathematically describes the rate sensing utilized. Additionally it describes the known and proven testing and calibration process utilized on DTGs.

\subsection{Dynamics of Dynamically Tuned Gyroscopes}

There is a large body of research pertaining to the Dynamically Tuned Gyroscope. Howe and Savet [13] were the first to include the inertial effects of the gimbal dynamics in their model and presented the first version of the tuning condition for 
DTGs. An analysis of the dynamics of the DTG was performed by Craig [6] using Newton-Euler Equations which extended this modeling approach and included damping coefficients for the torsional flexures. A transfer function model of a DTG was developed which can be used for both rebalance loop servo design and analysis of errors caused by angular inputs acting at twice the spin frequency. This model has served as a benchmark for DTG analysis and control system design. However, this analysis contains some simplifications and the system of equations is transformed into non-spinning coordinates early in the analysis. Transforming the system of equations too early when performing a small angle analysis can result in the loss of important terms. A second paper by Craig [7] discusses possible DTG rotor drift errors and the sensitivity of the rotor to $2 \omega_{z}$ vibrations, linear accelerational loads, and other inputs.

\subsubsection{Tuning Condition of the DTG}

The models used to design control systems for DTGs generally assume that the system is tuned. The gimbal dynamics are ignored with the model representing a freely spinning rotor or free gyro. The following is a simple illustration adapted from Cain [5], pages 25-30, of how the tuning equation for the DTG is calculated. It employs a derivation which is based on the analyses by Craig [8]. When the rotor is given a positive rotation about the $\mathrm{y}$ axis of the gimbal, the resulting moment is:

$$
M_{r y}=2 K_{y} \theta_{y}
$$

where $K_{y}$ represents the stiffness of one pivot along the y axis and $\theta_{y}$ represents the angle by which the rotor is rotated with respect to the gimbal.

Cain [5] has shown that for very small angular deflections of the rotor, the moments about the $\mathrm{x}$ axis of the rotor can be calculated to be:

$$
-2 K_{x} \theta_{x}-M_{r x}=I_{g x} \dot{\omega}_{g x}+\left(I_{g z}-I_{g y}\right) \omega_{g y} \omega_{g z}
$$


This expression can be simplified to give

$$
M_{r x}=-2 K_{x} \theta_{x}-I_{g x} \dot{\omega}_{g x}+\left(I_{g y}-I_{g z}\right) \omega_{g y} \omega_{g z}
$$

where the subscript $g$ refers to the gimbal, $I$ represents the mass moment of inertia and $\omega_{g x}, \omega_{g y}$, and $\omega_{g z}$ represent the gimbal angular rates. When the assumptions that the torsional springs connecting the rotor and gimbal lie along the gimbal y axis, and the springs connecting the gimbal to the shaft lie along the gimbal $\mathrm{x}$ axis are made, then the gimbal angular rates can be calculated in terms of the gimbal angles, $\theta_{x}$ and $\theta_{y}$, by rotating the angular rate of the shaft, $\omega_{z}$, about the $\mathrm{x}$ axis of the gimbal and then adding the rate of the gimbal about this axis. The net result is:

$$
\begin{aligned}
\dot{\boldsymbol{\omega}}_{g} & =\left(\begin{array}{c}
\dot{\theta_{x}} \\
0 \\
0
\end{array}\right)+\left(\theta_{x}\right)_{x}\left(\begin{array}{c}
0 \\
0 \\
\omega_{z}
\end{array}\right) \\
& =\left(\begin{array}{c}
\dot{\theta_{x}} \\
S_{\theta_{x}} \omega_{z} \\
C_{\theta_{x}} \omega_{z}
\end{array}\right)
\end{aligned}
$$

Then assuming small angles, the gimbal angular rates can be substituted into Equation 4.3 to give

$$
M_{r x}=-2 K_{x} \theta_{x}-I_{g x} \ddot{\theta}_{x}+\left(I_{g y}-I_{g z}\right) \dot{\theta}_{y} \omega_{z}
$$

When $\theta_{x}$ is defined in terms of an initial offset, $\theta_{x, o}$,

$$
\theta_{x}=\theta_{x, o} \cos \omega_{z} t
$$

The derivative, and second derivative, of $\theta_{x}$ with respect to time are then

$$
\dot{\theta_{x}}=-\omega_{z} \theta_{x, o} \sin \omega_{z} t
$$


and

$$
\ddot{\theta_{x}}=-\omega_{z}^{2} \theta_{x, o} \cos \omega_{z} t
$$

By realizing the angle $\theta_{y}$ has a 90 degree phase shift from $\theta_{x}$, it can then be defined by:

$$
\theta_{y}=\theta_{x, o} \sin \omega_{z} t
$$

Substituting these relationships into Equation 4.5 and 4.1 and simplifying gives

$$
\begin{gathered}
M_{r x}=\left[\left(I_{g x}+I_{g y}-I_{g z}\right) \omega_{z}{ }^{2}-2 K_{x}\right] \theta_{x, o} \cos \omega_{z} t \\
M_{r y}=2 K_{y} \theta_{x, o} \sin \omega_{z} t
\end{gathered}
$$

Note that Equations 4.6 and 4.7 are written in the local coordinate system of the rotor. It is also possible to transform these expressions to a non-spinning coordinate system by a rotation of $-\omega_{z} t$ about the $\mathrm{z}$ axis of the rotor. The resulting expressions for the torques on the rotor are then:

$$
\begin{gathered}
M_{x}=\left[\left(I_{g x}+I_{g y}-I_{g z}\right) \omega_{z}{ }^{2}-2 K_{x}\right] \theta_{x, o} \cos ^{2} \omega_{z} t-2 K_{y} \theta_{x, o} \sin ^{2} \omega_{z} t \\
M_{y}=\left[\left(I_{g x}+I_{g y}-I_{g z}\right) \omega_{z}{ }^{2}-2 K_{x}\right] \theta_{x, o} \cos \omega_{z} t \sin \omega_{z} t+2 K_{y} \theta_{x, o} \cos \omega_{z} t \sin \omega_{z} t
\end{gathered}
$$

The above expressions can be simplified to give

$$
\begin{aligned}
M_{x}= & \frac{1}{2}\left[\left(I_{g x}+I_{g y}-I_{g z}\right) \omega_{z}^{2}-2\left(K_{x}+K_{y}\right)\right] \theta_{x, o} \\
& +\frac{1}{2}\left[\left(I_{g x}+I_{g y}-I_{g z}\right) \omega_{z}^{2}-2\left(K_{x}+K_{y}\right)\right] \theta_{x, o} \cos 2 \omega_{z} t \\
M_{y}= & \frac{1}{2}\left[\left(I_{g x}+I_{g y}-I_{g z}\right) \omega_{z}^{2}-2\left(K_{x}+K_{y}\right)\right] \theta_{x, o} \sin 2 \omega_{z} t
\end{aligned}
$$

Ideally for the DTG to operate as a true "free gyro" the moments on the rotor would be zero; however, it would be difficult to make all the terms in the above expressions equal zero. To address this there are a set of five conditions listed in the literature that must be met for the DTG to be considered a free gyro. First, the gyro must be operated at the tuned speed. The theory and determination of 
the tuned speed has been discussed in Chapter 2. The tuning condition shown in Equation 2.24 can be found by forcing the first term of Equation 4.10 to equal zero.

$$
\omega_{s}=\omega_{z}=\sqrt{\frac{K_{x}+K_{y}}{I_{g x}+I_{g y}-I_{g z}}}
$$

The second term of Equation 4.10 relates to gimbal flutter on the rotor. This term cannot vanish but can be minimized by applying the remaining four tuning conditions for consideration as a free gyro. The analyses of Staley [23] and Lawrence [15] agree with this conclusion. Along with satisfying the first tuning condition described above, damping torques may not act on the rotor. To satisfy this condition, the stiffness about the y axis of the gimbal, $K_{y}$, should also be zero. If this tuning condition was met it would then be possible to eliminate the remaining terms in the above expressions. However, this condition is generally ignored because the non-symmetry of the springs results in other error terms. As discussed by Lawrence [15], the fourth condition requires that

$$
I_{g x}+I_{g y}-I_{g z}=0
$$

If the gimbal was a thin disk, this condition would be satisfied, but would result in an infinite tuning speed. The final condition states that the rotor should also be a thin disk or

$$
I_{r y}+I_{r z}-I_{r x}=0
$$

where $r$ represents the rotor. However, this is not practical; the result would cause the rotor to nutate and precess.

The quality of a DTG can be represented by a term called the Figure of Merit, $F_{m}$, which relates the inertias of the gimbal to the spin inertia of the rotor. Mathematically,

$$
F_{m}=\frac{I_{r z}}{I_{g x}+I_{g y}-I_{g z}}
$$

If the fourth tuning condition was satisfied, Equation 4.13, an infinite Figure of Merit would result, which is impossible. In practice, the gimbal is small compared 
to the spin inertia of the rotor. Thus, giving the largest Figure of Merit possible. "The $F_{m}$ of a typical high quality gyro is in the range of 200 to 300 ", as stated by Cain [5].

\subsubsection{DTG Control System Design}

As stated above, when developing models to design control systems for DTGs, the system is assumed to be tuned with the DTG acting as a free gyro. The following is a derivation adapted from Ower [17], pages 33-36, for DTG Control System Design. The rotor dynamics can be described using Euler's equation, as shown by Goldstein [11]:

$$
\begin{aligned}
& I_{r x} \dot{\omega}_{r x}-\omega_{r y} \omega_{r z}\left(I_{r y}-I_{r z}\right)=T_{r x} \\
& I_{r y} \dot{\omega}_{r y}-\omega_{r x} \omega_{r z}\left(I_{r z}-I_{r x}\right)=T_{r y} \\
& I_{r z} \dot{\omega}_{r z}-\omega_{r x} \omega_{r y}\left(I_{r x}-I_{r y}\right)=T_{r z}
\end{aligned}
$$

where $\omega_{r x}, \omega_{r y}, \omega_{r z}$ are rotor angular velocities expressed in the $\mathrm{x}, \mathrm{y}$, and $\mathrm{z}$ axes of its body frame; $I_{r x}, I_{r y}, I_{r z}$ are mass moments of inertia, expressed in the rotor body frame (the principal axis of inertia is assumed to be aligned with the body frame); and $T_{r x}, T_{r y}, T_{r z}$ are rotor control torques expressed in the body frame.

If inertias about the two non-spin axes $\left(I_{r x}, I_{r y}\right)$ are assumed equal, Equations 4.16 simplify to:

$$
\begin{aligned}
& \left.\begin{array}{l}
I_{r t} \dot{\omega}_{r x}+\left(I_{r z}-I_{r t}\right) \omega_{r y} \omega_{r z}=T_{r x} \\
I_{r t} \dot{\omega}_{r y}-\left(I_{r z}-I_{r t}\right) \omega_{r x} \omega_{r z}=T_{r y}
\end{array}\right\} \quad \begin{array}{r}
\text { transverse-axes dynamics } \\
I_{r z} \dot{\omega}_{r z}=T_{r z}
\end{array} \quad \text { spin dynamics }
\end{aligned}
$$

where

$$
I_{r t}=I_{r x}=I_{r y}
$$

If the rotor spin rate $\omega_{r z}$ is constant at the tuned speed $\omega_{s}$, the first two equations 
in 4.17 become decoupled from the spin axis motion. Control can then be split between transverse-axes control and spin control.

For the transverse-axes dynamics a complex analysis can be used. The complex rotor angular velocity $\omega_{r}$ and the complex control torque $T_{r}$ are:

$$
\begin{aligned}
& \omega_{r}=\omega_{r x}+\mathrm{j} \omega_{r y} \\
& T_{r}=T_{r x}+\mathrm{j} T_{r y}
\end{aligned}
$$

the first equation in 4.17 can be added to $\mathrm{j}=\sqrt{-1}$ times the second equation in 4.17 . The result is the simple complex equation:

$$
I_{r t} \dot{\omega}_{r}-\mathrm{j}\left(I_{r z}-I_{r t}\right) \omega_{s} \omega_{r}=T_{r}
$$

Flexure stiffness and gimbal dynamics are included in the transverse-axes dynamic equations of 4.17. More detailed linearized equations of motion as shown by Craig [6] take the following form:

$$
\begin{aligned}
& m_{1} \ddot{\theta_{x}}+c \dot{\theta_{y}}+K_{x} \theta_{x}=T_{g x}(t) \\
& m_{2} \ddot{\theta_{y}}+c \dot{\theta_{x}}+K_{y} \theta_{y}=T_{g y}(t)
\end{aligned}
$$

where $\theta_{x}$ is the $\mathrm{x}$ axis gimbal angle, $\theta_{y}$ the $\mathrm{y}$ axis gimbal angle, and $T_{g x}, T_{g y}$ the generalized control torques acting about the two gimbal angles. $m_{1}, m_{2}$ are inertia coefficients, $c$ a common damping term, and $K_{x}, K_{y}$ are stiffness terms. The stiffness, damping and inertia terms depend on the rotor and gimbal mass properties, flexure stiffness, and spin rate. This coupled TDOF system there will have two complexconjugate pairs of eigenvalues. These are apparent in the spinning body frame. An alternative form is one that describes the motion using case-referenced tilt angles. As shown by Craig [6], these equations can be expressed in non-spinning coordinates, where most analyses show there are two characteristic roots, one at the nutation frequency, and the other at zero frequency.

Lawrence [16] performed a general analysis using the state space method and identified three periodic oscillations of the rotor orientation. These frequencies con- 
sisted of the nutation frequency of the rotor, a term which would be zero if the system was tuned, and a frequency at twice the spin frequency.

Staley [23], using Lagrange's Equations, retained all terms in the dynamic equations through the transformation process, keeping the results in spinning coordinates until the last step. Importantly this resulted in the observation of four unique characteristic frequencies. The first and second occur at nearly twice the spin frequency, representing a long term precession, and the nutation frequency as previously identified. A third appears as a beat frequency between the nutation frequency and the twice spin frequency term. A final mode occurs at a very low frequency, but not zero frequency as the linear model from Craig [6] indicates.

In the course of the dynamic investigations of DTGs, simplifying assumptions have been made including: deflections consist of only small angles; symmetric rotor and gimbal; stiffness of the torsional springs remains constant; case of the gyro is fixed to an inertial frame; and the system consists of gimbal rings and rotor only, implying that the input shaft speed is constant. Given the large tilts and de-tuned speed operation of the GyroWheel ${ }^{\text {TM }}$ not all of these assumptions are valid, especially when considering that the larger angular tilts make the small angle approximations used in DTG models less valid, and almost all models assume operation at tuned speed.

\subsubsection{Gyroscope Error Terms}

This subsection discussing gyroscope error terms as reviewed by Titterton and Weston [25], pages 71-73. It is well established that all gyroscopic sensors are subject to errors which affect the accuracy with which the angle of rotation or applied turn rate is measured. Erroneous torques, that are often caused by design limitation and constructional deficiencies, are known to act on the rotors of all mechanical gyroscopes. These imperfections give rise to precession of the rotor, resulting in a 'drift' in the reference direction defined by the spin axis of the rotor. For a restrained 
gyroscope, these erroneous torques produce a 'bias' on the measurement of angular rate. Titterton and Weston [25] highlight seven major sources of error that arise in mechanical gyroscopes. These include:

\section{Fixed Bias (g independent bias)}

When, in the absence of an applied input rotation, there is a sensor output present, this sensor input is called a fixed bias error. This may be a consequence of any variety of effects, including residual torques from flexible leads within the sensor, spurious magnetic fields and temperature gradients, which produce such biases. It is important to note that the size of the bias is independent of any motion the gyroscope is subjected to. It is also referred to as the acceleration (or g) independent bias and is generally expressed in units of degrees per hour $(\% / \mathrm{h})$.

\section{Acceleration Dependent Bias ( $\mathrm{g}$ dependent bias)}

These biases are proportional to the magnitude of an acceleration applied to the gyroscope. These types of errors arise in spinning mass gyroscopes and are the result of mass unbalance in the rotor suspension. These terms relate accelerations in each principal axis of the gyroscope to errors in the measurement of the turn rate. In the presence of a constant acceleration, a fixed bias in the measured rate occurs. These error coefficients have units of $(\% / \mathrm{h} / \mathrm{g})$.

\section{Anisoelastic Bias $\left(\mathrm{g}^{2}\right.$ dependent bias)}

These biases are proportional to the product or combination of acceleration along orthogonal pairs of axes. These biases occur most frequently in spinning mass gyroscopes because the gyroscope rotor suspension structure have finite tolerances that are generally unequal in different directions. Up to nine scparate $\mathrm{g}^{2}$ terms can be defined for a DTG, but all are due to an acceleration being sensed by two axes simultaneously. These error coefficients have units of $\left(\% / \mathrm{h} / \mathrm{g}^{2}\right)$. 


\section{Anisoinertia Errors}

These errors arise in spinning mass gyroscopes due to inequalities in moments of inertia about different axes. If the rotor is driven by a hysteresis motor these errors are frequency sensitive due to the elastic coupling between the magnetic ring on the rotor and the rotating magnetic field. The resulting errors are proportional to the product of angular rates applied about orthogonal axes pairs. These error coefficients can be expressed in units of $\left({ }^{\circ} / \mathrm{h} /(\mathrm{rad} / \mathrm{s})^{2}\right)$.

\section{Scale Factor Errors}

These errors are related to the ratio utilized to correlate changes in the output signal to changes in the input rate being measured, and are often commonly expressed as a ratio of output error to input rate, in parts per million (ppm). Scale factor nonlinearity and scale factor asymmetry can result in additional errors. Scale factor non-linearity is defined as any systematic deviations from the non-linear function that is fitted to the measurements in order to relate the output signal to the applied rate. Scale factor asymmetry is defined as any difference in the magnitude of the output signal for equal rotations of the sensor in opposite directions. Scale factor asymmetry often relates to temperature changes that subsequently cause changes in the magnetic flux.

\section{Cross-coupling Errors}

There are erroneous gyroscope outputs that occur as a result of the gyroscope being sensitive to turn rates about axes that are normal to the input axes. These errors occur as a result of non-orthogonality of the sensor axes and may also be expressed as ppm or a percentage of the applied angular rate.

\section{Angular Acceleration Sensitivity}

This type of error is also known as the gyroscopic inertial error because it is caused by the fact that all mechanical gyroscopes are sensitive to angular acceleration due 
to the inertia of their rotor. These errors are especially important in wide bandwidth applications because they increase with increasing frequency of input motion.

It is of importance to note that each of the errors described will, in general, include some or all of the following components:

- Fixed or repeatable terms;

- Temperature induced variations;

- Switch-on to switch-on variations; and

- In-run variations.

For example, when the gyroscope provides a measurement of angular rate, this reading will include:

1. a predictable bias component that is present each time the sensor is switched on and can therefore be corrected;

2. a temperature dependent bias component which can be corrected through calibration;

3. a random bias which varies from gyroscope switch-on to switch-on but remains constant for any single run; and

4. an in-run random bias which varies throughout a single run.

As discussed by Titterton and Weston [25], the fixed components of error, including the temperature induced variations, can often be corrected. This leaves only errors attributed to switch-on to switch-on variation and in-run effects, which are related to the random effects caused by instabilities within the gyroscope. Assuming that the systematic errors can be compensated for, the switch-on to switch-on and in-run variations will exert the main influence on the performance of the inertial system in which the gyroscope is installed.

\subsubsection{Errors Specific to the DTG}

As discussed by Titterton and Weston [25], the following is a list of errors experienced specifically by DTGs as categorized above: 


\section{DTG g Insensitive Bias}

This is mainly the result of spurious internally generated magnetic fields that interact with both the rotor mounted torque motor magnet and the re-balance loop biases. This error often includes the effects of tuning errors and gimbal damping.

\section{DTG g Sensitive Bias}

This is caused by mass unbalance of the rotor assembly and geometrical imperfections in the torsional elements. As highlighted by Hartl [12], further elaboration on two of the primary g dependent terms, the mass unbalance and the quadrature are provided below. As shown in Figure 4.1 from Hartl [12], a DTG is oriented such that the spin axis is perpendicular to the gravity vector, and the $\mathrm{x}$ input axis is aligned with the gravity vector. In an ideal setting, the spinning mass is perfectly balanced in the plane of the wheel. However, any imperfection in mass distribution results in a $\mathrm{g}$ sensitive bias which can be modeled as a point mass a distance, $d$, along the spin axis direction, causing a torque that is proportional to the mass and lever arm to occur. Because this torque acts in the y direction of the DTG, a precessional rate appears about the $\mathrm{x}$ input axis (fundamental law of precession) and is referred to as mass unbalance drift $\left(M U_{x}\right)$. The same logic can be used to establish the existence of a mass unbalance that occurs when the y input axis is aligned with gravity $\left(M U_{y}\right)$. Both problems arise from the poor distribution of mass in the spin direction. Thus, the terms are almost always similar in magnitude, assuming that the scale factors for both axes are comparable. It is almost impossible to affect one mass unbalance term without changing the other one.

The second major $g$ dependent effect is known as quadrature mass unbalance $\left(Q_{x}\right.$ and $\left.Q_{y}\right)$. This occurs when the flexures generate a torque when loaded axially, which subsequently leads to an acceleration sensitive bias about the opposite axis to the axis along which the acceleration is acting. There are four flexures incorporated in a DTG: two of them connect the rotor to the gimbal; and the other two connect 


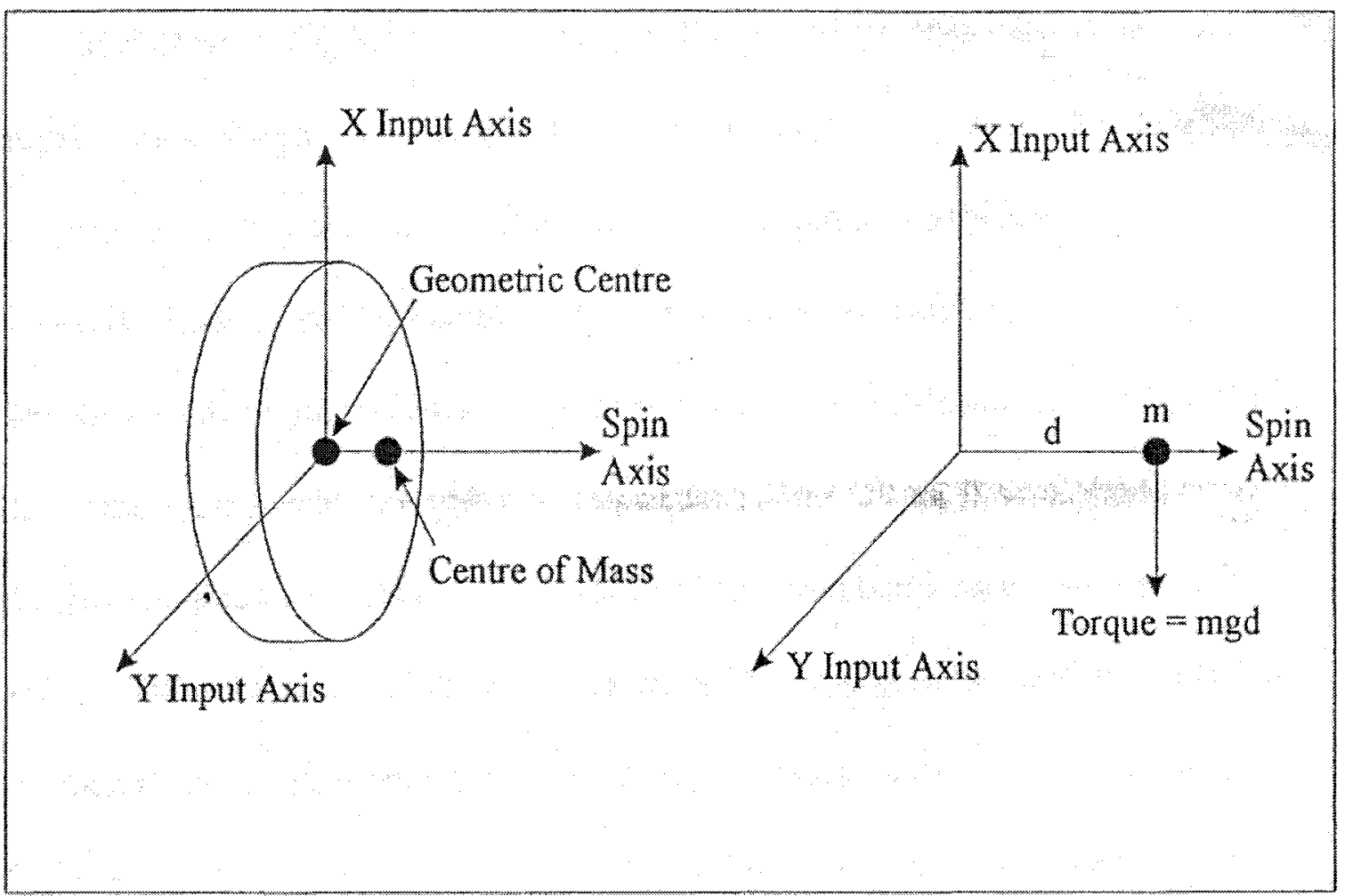

Figure 4.1: Visualization of the Mass Unbalance Error Term in a DTG, from Hartl $[12]$ 
the gimbal to the shaft. In order to understand this error term one needs to assume that one of the four flexures has a defect in construction and that the other three flexures are constructed exactly the same. In reality, all flexures would have some varying imperfections, however minor, and as a result would all contribute uniquely to the quadrature mass unbalance.

Figure 4.2 from Hartl [12] helps to illustrate the mechanics leading to the generation of this error. If one considers a DTG wheel rotating through $360^{\circ}$, at its starting point, the defective flexure is lying horizontally, without any longitudinal force acting upon it. When the DTG wheel rotates a quarter turn, the defective flexure goes into tension as a result of the weight of the gimbal-rotor assembly. The tension causes the flexure to twist and generates a torque. After half a revolution, the defective flexure is horizontal and the generated torque returns to zero. At the three quarter turn mark, the defective flexure is again stressed, but in compression instead of tension. The stress due to compression causes a torque to be generated equal in magnitude, but opposite in sense to the torque generated due to the tensile stress from the previous half turn. However, because the rotor position has changed by $180^{\circ}$, the torque still acts in the same absolute direction. Over the course of a full turn, the torque varies continually between zero and $T_{\max }$, and an overall non-zero mean torque exists. An acceleration along the $\mathrm{x}$ axis causes a torque to about the $\mathrm{x}$ axis, resulting in a drift along the $\mathrm{y}$ axis and vice versa.

\section{DTG Anisoelastic Bias}

Anisolelastic bias is the result of unequal compliance of the rotor assembly in all three axes: $\mathrm{x}, \mathrm{y}$, and $\mathrm{z}$. Anisoelasticity as discussed by Hartl[12] can arise when the clasticity in a specific axial direction does not correlate with the radial clasticity. As shown in Figure 4.3 from Hartl [12], a point mass is shown to be suspended by two springs. The springs are shown to be positioned neutrally and are at right angles to each other. If one of the springs, i.e. $K_{y}$, is less elastic than the other and an 


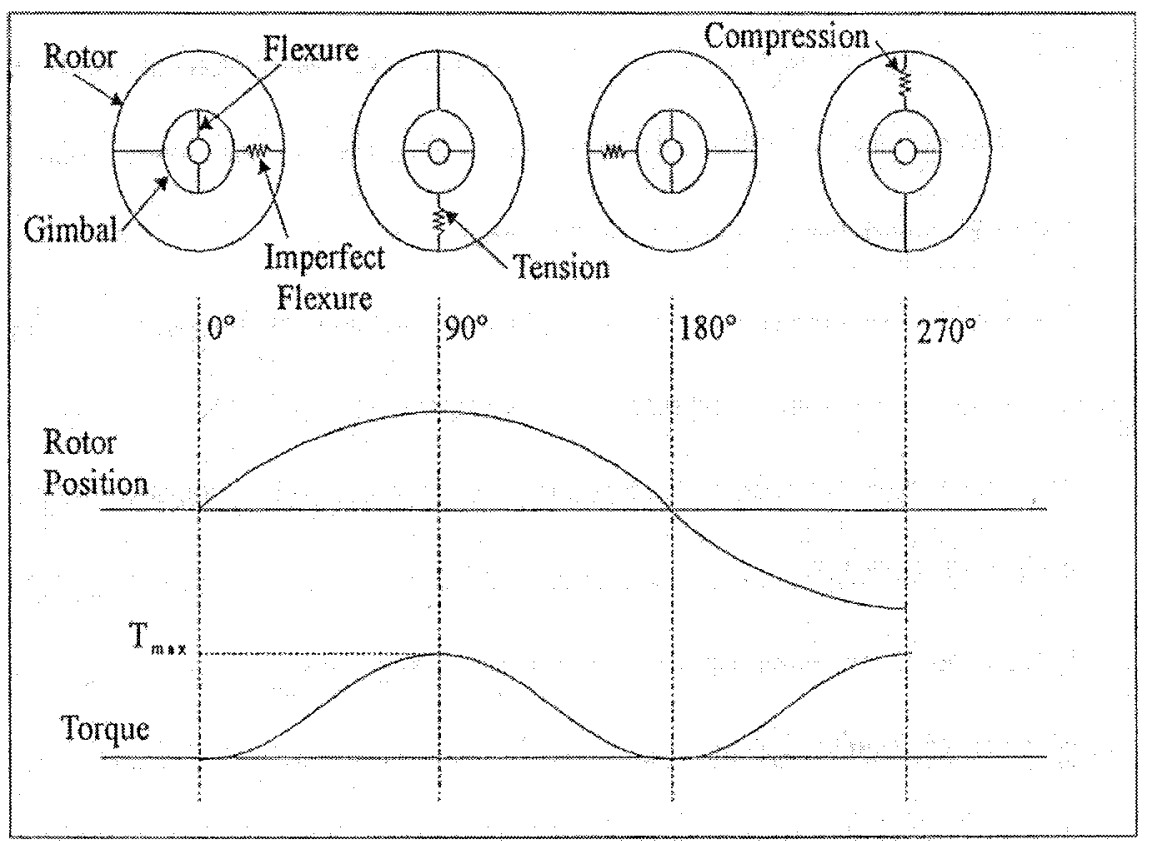

Figure 4.2: Explanation of the Quadrature Error Term in a DTG, from Hartl [12]

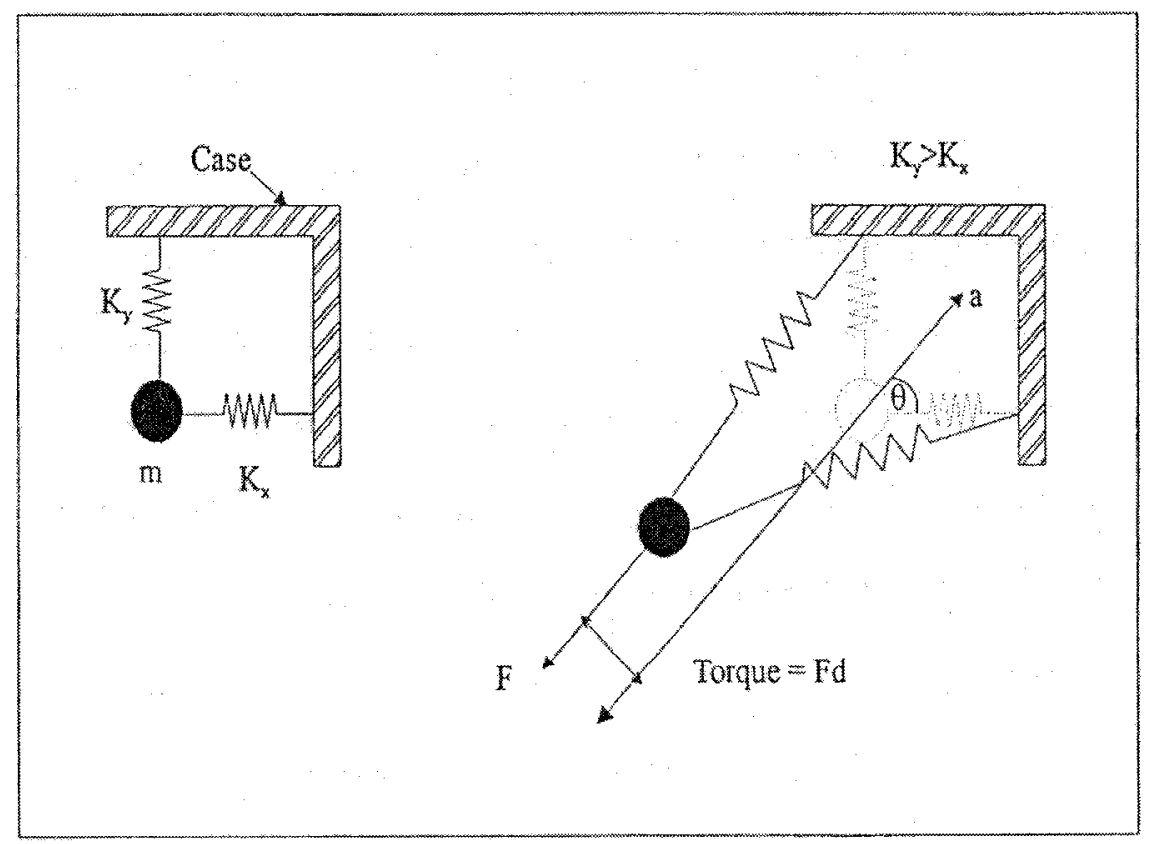

Figure 4.3: Explanation of Anisoelastic Torque in a DTG, from Hartl [12] 
acceleration is applied to the mass, the net result is that the mass deviates from the line of action of the force. As a result the mass is displaced a perpendicular distance, $d$, from the force, and a torque arises in accordance with:

$$
T=\frac{1}{2} m^{2} a^{2}\left[\frac{1}{K_{y}}-\frac{1}{K_{x}}\right] \sin 2 \theta
$$

When this equation is examined further it can be determined that $T$ is maximum when $\theta$ is $45^{\circ}$. This is important because this directly affects DTG test definitions discussed in Section 4.2. It is also important to note that the spring mass system represented in Figure 4.3 is analogous to the DTG flexure system. Thus any acceleration acting along two separate axes, results in the introduction of torques that can induce drift errors that would require compensation. This is usually a non-issue in higher quality DTGs because these second order drift terms are often negligible. Unfortunately this is often not the case for every DTG, and individual DTGs must undergo assessment in a controlled laboratory environment before drawing such a conclusion.

\section{DTG Anisoinertia Bias}

This is generated from differences in the rotor inertias of the $\mathrm{x}, \mathrm{y}$, and $\mathrm{z}$ axes and are frequency sensitive.

\section{DTG Scale Factor Errors}

These are generally the result of thermal changes that occur in the magnets and coils of a DTG re-balance system.

\section{DTG Zero-mean Random Bias}

As an example, when changes in spin motor - shaft orientation caused by variations in the bearing pre-load occur, error torques result, which are a form of zero-mean random bias. 
In summary, the DTG is sensitive to both linear and angular accelerations, as well as vibratory motion, stray magnetic fields, and temperature changes. Each of these can cause errors in measurements. Importantly, the DTG is sensitive to vibrations mainly at integer multiples of the spin speed, most notably the two times spin frequency. In order to give a fixed bias, the interaction between vibration about the input axis and the gimbal angular momentum must be rectified.

\subsubsection{DTG Rate Sensing}

As discussed by Ower [17], the external rates can be extracted from the rate gyro by examining the equations of motion of the transverse axes from Craig [6]:

$$
\begin{aligned}
& I_{r t} \ddot{\phi}_{x}+I_{r z} \omega_{z} \dot{\phi}_{y}+I_{r t} \dot{\omega}_{c X}+I_{r z} \omega_{z} \omega_{c Y}=T_{c X} \\
& I_{r t} \ddot{\phi}_{y}+I_{r z} \omega_{z} \dot{\phi}_{x}+I_{r t} \dot{\omega}_{c Y}+I_{r z} \omega_{z} \omega_{c X}=T_{c Y}
\end{aligned}
$$

where $\phi_{x}, \phi_{y}$ represent the case-reference tilt angle of the momentum axis; $\omega_{c X}, \omega_{c Y}$ represent the angular velocity of the spacecraft relative to the inertial frame; and $T_{c X}, T_{c Y}$ represent the case-referenced control torque. To generate the control torques so that the case referenced tilt angles remain at null, a rebalance control system is employed. Note that the bandwidth of the rebalance control system is normally greater than $10 \mathrm{~Hz}$.

The frequency of the gyro control system bandwidth is higher than the frequency of the torques that are induced on the rotor by the motion of the base body. Due to this bandwidth separation, the first three terms in Equations 4.24 can be decmed negligible, allowing for the easy determination of the external rates. The equations of motion simplify to:

$$
\begin{aligned}
& I_{r z} \omega_{z} \omega_{c Y} \approx T_{c X} \\
& I_{r z} \omega_{z} \omega_{c X} \approx T_{c Y}
\end{aligned}
$$


Isolating for the external rates gives:

$$
\left[\begin{array}{l}
\omega_{c X} \\
\omega_{c Y}
\end{array}\right] \approx \frac{1}{I_{r z} \omega_{z}}\left[\begin{array}{l}
T_{c Y} \\
T_{c X}
\end{array}\right]
$$

Because the control torques are proportional to the current in each torquer coil, the control torques can therefore be determined using the following relationship:

$$
\left[\begin{array}{c}
T_{c X} \\
T_{c Y}
\end{array}\right]=k_{\mathrm{trq}}\left[\begin{array}{c}
i_{x} \\
i_{y}
\end{array}\right]
$$

where $k_{\text {trq }}$ describes the factor by which coil current is converted to torque. $k_{\text {trq }}$ is based on the interaction of the magnetic dipole moment that is created by the current in the coil in addition to its interaction with the radial magnetic field created by the rotor permanent magnets.

Equation 4.27 can be substituted into Equation 4.26 to provide an approximation of the external rates in relation to the torque coil current:

$$
\left[\begin{array}{c}
\omega_{c X} \\
\omega_{c Y}
\end{array}\right] \approx \frac{k_{\operatorname{trq}}}{I_{r z} \omega_{z}}\left[\begin{array}{l}
i_{x} \\
i_{y}
\end{array}\right]
$$

Development of the complete mathematical description of a DTG is a lengthy and arduous task that is beyond the scop of this thesis. This area of research has been mentioned in brief above, and an excellent development can be found in the IEEE Specification Format Guide and Test Procedure for Two-Degree-of-Freedom Dynamically Tuned Gyros [14], which commences with much of Savet and Howe's, and Craig's, but also includes many system level block diagrams which help clarify some of the mathematics.

In general a rebalance torque is applied to the DTG by means of torquer coils. Therefore, by measuring the current applied to the torquer coils in order to keep the rotor at null, the torque and hence the drift experienced by the gyro can be determined during closed loop testing. The model equations for a DTG relate the 
torquer current to the algebraic sum of the applied input and the error parameters. The general rebalance equations, from Hartl [12] can be written as follows:

$$
\begin{aligned}
k_{\text {scale }, x} i_{x} & =\omega_{c X}+D(x)_{F}+D(x)_{X} a_{X}+D(x)_{Y} a_{Y}+D(x)_{Z} a_{Z} \\
& +D(x)_{X X} a_{X}^{2}+\left[D(x)_{Y Y} a_{Y}^{2}\right]+D(x)_{Z Z} a_{Z}^{2} \\
& +D(x)_{X Y} a_{X} a_{Y}+D(x)_{X Z} a_{X} a_{Z}+D(x)_{Y Z} a_{Y} a_{Z} \\
k_{\text {scale }, y} i_{y} & =\omega_{C Y}+D(y)_{F}+D(y)_{Y} a_{Y}+D(y)_{X} a_{X}+D(y)_{Z} a_{Z} \\
& +\left[D(y)_{X X} a_{X}^{2}\right]+D(y)_{Y Y} a_{Y}^{2}+D(y)_{Z Z} a_{Z}^{2} \\
& +D(y)_{Y X} a_{X} a_{Y}+D(y)_{X Z} a_{X} a_{Z}+D(y)_{Y Z} a_{Y} a_{Z}
\end{aligned}
$$

Equation 4.29 fully describes the $\mathrm{X}$ input axis characteristics, while Equation 4.30 applies to the $\mathrm{Y}$ input axis. Note that these definitions of $i_{x}$ and $i_{y}$ are opposite from the conventional rate sensing definitions of the two currents in gyro calibration. The above definitions were adopted based on previously defined conventions for GyroWheel ${ }^{T M}$ momentum actuation. The right side of the equations account for the applied rates, $\omega$, and the associated anisoinertia and cross-coupling errors, as well as the other error parameters affecting that axis. Additionally $k_{\text {scale }, x}$ and $k_{\text {scale }, y}$ may contain scale factor errors as defined above.

The notation used in these equations allows all error terms to be expressed in a common fashion. However, by convention certain terms are given specific names. $D(x)_{F}$ is the absolute bias of the $\mathrm{X}$ axis and is often given the name, $B_{x} . D(x)_{X}$ is the mass unbalance terms as discussed in Section 4.1.4, known more commonly as $M U_{x}$, while $D(x)_{Y}$ is the quadrature term, often referred to as $Q_{x}$. The $\mathrm{Y}$ axis drift terms follow similar conventions. Therefore Equations 4.29 and 4.30 can be 
rewritten as:

$$
\begin{aligned}
k_{\text {scale, }, i_{x}} & =\omega_{c X}+B_{x}+M U_{x} a_{X}+Q_{x} a_{Y}+D(x)_{Z} a_{Z} \\
& +D(x)_{X X} a_{X}^{2}+\left[D(x)_{Y Y} a_{Y}^{2}\right]+D(x)_{Z Z} a_{Z}^{2} \\
& +D(x)_{X Y} a_{X} a_{Y}+D(x)_{X Z} a_{X} a_{Z}+D(x)_{Y Z} a_{Y} a_{Z} \\
k_{\text {scale }, y} i_{y} & =\omega_{c Y}+B_{y}+M U_{y} a_{Y}+Q_{y} a_{X}+D(y)_{Z} a_{Z} \\
& +\left[D(y)_{X X} a_{X}^{2}\right]+D(y)_{Y Y} a_{Y}^{2}+D(y)_{Z Z} a_{Z}^{2} \\
& +D(y)_{Y X} a_{X} a_{Y}+D(y)_{X Z} a_{X} a_{Z}+D(y)_{Y Z} a_{Y} a_{Z}
\end{aligned}
$$

These notations are used interchangeably in the literature. The two second order drift terms within square brackets cannot be determined utilizing static $1 \mathrm{~g}$ testing and are therefore assumed to be negligible during such tests. Wilkinson [31] has shown that this assumption is valid for high quality DTGs.

\subsection{Testing and Calibration of Dynamically Tuned Gyroscopes}

As shown above it is possible to represent the behaviour or performance of a DTG with a mathematical expression that will closely describe the behaviour of the instrument. The main purpose of testing an inertial sensor is to evaluate the cocfficients, or various drift or bias terms, of these equations so that the performance of a sensor can be predicted for particular operating parameters. Having determined the performance figures by characterizing the gyroscope, any systematic errors may be compensated for, thus enhancing the rate sensing accuracy. Other purposes of testing are to enable the output signals to be calibrated and to understand the behaviour of the device in various situations and environments.

The testing is performed in a controlled environment where as many variables as possible which may affect the instrument have either been eliminated, controlled, 
or measured. The testing can generally be divided into two categories: static; and dynamic testing. Static testing implies the DTG is not moving relative to the Earth, however it should be noted that the instrument is still moving with respect to inertial space and therefore experiences Earth rate and the effect of gravity. Dynamic testing involves applying a precisely known rate acceleration to the DTG, in addition to any effects due to Earth rate and gravity.

\subsubsection{Multi-Position Testing}

Because many of the error terms of the DTG mathematical expressions are based on sensitivities to gravity or to applied rates, it makes sense to place the DTG in orientations where these effects are either a maximum or minimum in order to determine the coefficient values. This can be accomplished by statically placing the instrument in a well known orientation defined relative to a precisely known Earth orientation. Also, most of the DTG error parameters involve acceleration terms along one of the gyro axes only. By precise orientation of the DTG with respect to the geographic frame of reference, and therefore the gravity vector, it is possible to eliminate the effect of many of these error terms depending on the orientation chosen. This precise positioning and DTG orientation can be structured so that an error term can have a full effect or no effect on the output. Additionally, since many error terms will change sign algebraically by inverting the DTG $180^{\circ}$ in the proper sense, the effect of that error term can be eliminated by algebraically adding the outputs obtained in the two opposite positions. Test positions are determined utilizing the mathematical model in order to place the DTG in orientations where the error parameters or coefficients have known effects. Positions $180^{\circ}$ inverted, that take advantage of the maximum positive or negative effects of error parametcrs on the output are crucial, as are positions that cause the error term to equal zero. For each given position, both the orientation of the gravity vector and the fraction of the Earth rate sensed by the DTG input axis is known. Mathematical expressions 
to solve for each of the error terms can then be determined by adding or subtracting equations from different positions.

With this in mind, Hartl [12] developed a series of positional tests for the DRDC Ottawa Navlab shown in Table 4.1. This is a complete test, comprised of 24 positions which can be used to determine all of the error parameters given in the general rebalance equations, Equations 4.29 and 4.30, for a DTG. It is often not required to determine all of the parameters in the general equation as some of the error terms may be insignificant especially in high quality gyroscopes, leading to a smaller subset of these positions being required. For example for very high quality gyros the second order terms $\left(\mathrm{g}^{2}\right)$ are often insignificant, and to determine just the bias and $\mathrm{g}$ sensitive terms, only 8 positions are required. The positional tests for 11 position testing is shown in Table 4.2 and are referred to later on in the testing and analysis in Chapters 5 and 6. 
Many mathematical derivations for multi-position testing exist, but given that there are certain differences between gyroscopes, testing equipment, and the definitions and conventions used during testing, equations need to be developed specific to the setup used to evaluate the gyroscope. In his Appendix A, Hartl [12] developed the multi-position equations for the CSG-2 gyro. Utilizing this development, the specific multi-position equations utilized for the GyroWheel ${ }^{\mathrm{TM}}$ were developed.

\subsection{Dynamics of the GyroWheel ${ }^{\mathrm{TM}}$ System}

The following section discusses the mathematical model of the GyroWheel ${ }^{\mathrm{TM}}$ system developed by de Carufel [9], including the assumptions made in the development of the model. The physical description of the GyroWheel ${ }^{\mathrm{TM}}$ is discussed in detail in Chapter 3.

\subsubsection{GyroWheel ${ }^{\text {TM }}$ Frames of Reference Definitions}

Figure 4.4 shows the three main reference frames for defining the motion of the GyroWheel $^{\text {TM }}$. Frame $B$ is the base frame (or spacecraft frame). The GyroWheel ${ }^{T M}$ frame $G$ is fixed with respect to the base frame $B$ and the rotor frame $R$ moves in three rotational DOF with respect to the GyroWheel ${ }^{\mathrm{TM}}$ frame $G$. As a convention, the leading superscript always refers to the frame in which the vector is represented.

The detailed motion of the GyroWheel ${ }^{\mathrm{TM}}$ is defined by considering two additional frames of reference defined as the shaft frame $S$ and the gimbal frame $g$ as shown in Figure 4.5

\subsubsection{GyroWheel ${ }^{\mathrm{TM}}$ Lagrangian Dynamics Formulation}

The GyroWheel ${ }^{\mathrm{TM}}$ could be assumed to be reacting as a simple double-gimbal momentum wheel, in which case the total momentum on the spacecraft, expressed 
Table 4.1: Definition of Gyroscope Axes Positions for a 24 Position Test

\begin{tabular}{|c|c|c|c|c|c|c|c|c|c|c|}
\hline \multicolumn{3}{|c|}{ Position } & \multicolumn{3}{|c|}{ Direction of Axes } & \multicolumn{3}{|c|}{ Acceleration Along } & \multicolumn{2}{|c|}{ Earth Rate Input } \\
\hline$\#$ & $\mathrm{Az}$. & Tilt & Spin & $\mathrm{X}$ & $Y$ & Spin & $\mathrm{X}$ & $\mathrm{Y}$ & $\mathrm{X}$ & $\mathrm{Y}$ \\
\hline 1 & 90 & 180 & $\mathrm{Up}$ & $\mathrm{N}$ & W & 1 & 0 & 0 & $\omega_{e} \cos (\lambda)$ & 0 \\
\hline 2 & 0 & 180 & $\mathrm{Up}$ & W & $\mathrm{S}$ & 1 & 0 & 0 & 0 & $-\omega_{e} \cos (\lambda)$ \\
\hline 3 & 270 & 180 & $\mathrm{Up}_{\mathrm{p}}$ & $\mathrm{S}$ & $\mathrm{E}$ & 1 & 0 & 0 & $-\omega_{e} \cos (\lambda)$ & 0 \\
\hline 4 & 180 & 180 & Up & $\mathrm{E}$ & $\mathrm{N}$ & 1 & 0 & 0 & 0 & $\omega_{e} \cos (\lambda)$ \\
\hline 5 & 180 & 270 & $\mathrm{~N}$ & $\mathrm{E}$ & Dn & 0 & 0 & -1 & 0 & $-\omega_{e} \sin (\lambda)$ \\
\hline 6 & 270 & 270 & $N$ & $\mathrm{Up}$ & $\mathrm{E}$ & 0 & 1 & 0 & $\omega_{e} \sin (\lambda)$ & 0 \\
\hline 7 & 0 & 270 & $\mathrm{~N}$ & $W$ & $\mathrm{Up}$ & 0 & 0 & 1 & 0 & $\omega_{e} \sin (\lambda)$ \\
\hline 8 & 90 & 270 & $\mathrm{~N}$ & Dn & $\mathrm{W}$ & 0 & -1 & 0 & $-\omega_{e} \sin (\lambda)$ & 0 \\
\hline 9 & 135 & 270 & $\mathrm{~N}$ & $\begin{array}{c}45^{\circ} \\
\text { Below E }\end{array}$ & $\begin{array}{c}45^{\circ} \\
\text { Below W } \\
\end{array}$ & 0 & $-\cos \left(\frac{\pi}{4}\right)$ & $-\cos \left(\frac{\pi}{4}\right)$ & $\begin{array}{c}-\omega_{e} \sin (\lambda) \\
\cos \left(\frac{\pi}{4}\right)\end{array}$ & $\begin{array}{c}-\omega_{e} \sin (\lambda) \\
\cos \left(\frac{\pi}{4}\right)\end{array}$ \\
\hline 10 & 225 & 270 & $\mathrm{~N}$ & $\begin{array}{c}45^{\circ} \\
\text { Above E } \\
\end{array}$ & $\begin{array}{c}45^{\circ} \\
\text { Below E }\end{array}$ & 0 & $\cos \left(\frac{\pi}{4}\right)$ & $-\cos \left(\frac{\pi}{4}\right)$ & $\begin{array}{c}\omega_{e} \sin (\lambda) \\
\cos \left(\frac{\pi}{4}\right) \\
\end{array}$ & $\begin{array}{c}-\omega_{e} \sin (\lambda) \\
\cos \left(\frac{\pi}{4}\right)\end{array}$ \\
\hline 11 & 315 & 270 & $N$ & $\begin{array}{c}45^{\circ} \\
\text { Above W }\end{array}$ & $\begin{array}{c}45^{\circ} \\
\text { Above E }\end{array}$ & 0 & $\cos \left(\frac{\pi}{4}\right)$ & $\cos \left(\frac{\pi}{4}\right)$ & $\begin{array}{c}\omega_{e} \sin (\lambda) \\
\cos \left(\frac{\pi}{4}\right)\end{array}$ & $\begin{array}{c}\omega_{e} \sin (\lambda) \\
\cos \left(\frac{\pi}{4}\right)\end{array}$ \\
\hline 12 & 45 & 270 & $\mathrm{~N}$ & $\begin{array}{c}45^{\circ} \\
\text { Below W }\end{array}$ & $\begin{array}{c}45^{\circ} \\
\text { Above W }\end{array}$ & 0 & $-\cos \left(\frac{\pi}{4}\right)$ & $\cos \left(\frac{\pi}{4}\right)$ & $\begin{array}{c}-\omega_{e} \sin (\lambda) \\
\cos \left(\frac{\pi}{4}\right)\end{array}$ & $\begin{array}{c}\omega_{e} \sin (\lambda) \\
\cos \left(\frac{\pi}{4}\right)\end{array}$ \\
\hline 13 & 90 & 0 & Down & $\mathrm{S}$ & $\mathrm{W}$ & -1 & 0 & 0 & $-\omega_{e} \sin (\lambda)$ & 0 \\
\hline 14 & 0 & 0 & Down & W & $\mathrm{N}$ & -1 & 0 & 0 & 0 & $\omega_{e} \sin (\lambda)$ \\
\hline 15 & 270 & 0 & Down & $\mathrm{N}$ & $\mathrm{E}$ & -1 & 0 & 0 & $\omega_{e} \sin (\lambda)$ & 0 \\
\hline 16 & 180 & 0 & Down & $\mathrm{E}$ & $S$ & -1 & 0 & 0 & 0 & $-\omega_{e} \sin (\lambda)$ \\
\hline 17 & 90 & 225.349 & $\begin{array}{c}\text { Polar } \\
\mathrm{N}\end{array}$ & $\begin{array}{l}\left(90^{\circ}-\lambda\right) \\
\text { Below N }\end{array}$ & W & $\sin (\lambda)$ & $-\cos (\lambda)$ & 0 & $\omega_{e} \cos (\lambda)$ & 0 \\
\hline 18 & 0 & 225.349 & $\begin{array}{c}\text { Polar } \\
\text { N }\end{array}$ & W & $\begin{array}{l}\left(90^{\circ}-\lambda\right) \\
\text { Above } \mathrm{S}\end{array}$ & $\sin (\lambda)$ & 0 & $\cos (\lambda)$ & 0 & 0 \\
\hline 19 & 270 & 225.349 & $\begin{array}{c}\text { Polar } \\
\mathrm{N}\end{array}$ & $\begin{array}{l}\left(90^{\circ}-\lambda\right) \\
\text { Above S }\end{array}$ & $\mathrm{E}$ & $\sin (\lambda)$ & $\cos (\lambda)$ & 0 & 0 & 0 \\
\hline 20 & 180 & 225.349 & $\begin{array}{c}\text { Polar } \\
\mathrm{N}\end{array}$ & $\mathrm{E}$ & $\begin{array}{l}\left(90^{\circ}-\lambda\right) \\
\text { Below N }\end{array}$ & $\sin (\lambda)$ & 0 & $-\cos (\lambda)$ & 0 & 0 \\
\hline 21 & 90 & 45.349 & $\begin{array}{c}\text { Polar } \\
\mathrm{S}\end{array}$ & $\begin{array}{l}\left(90^{\circ}-\lambda\right) \\
\text { Above } \mathrm{S}\end{array}$ & W & $-\sin (\lambda)$ & $\cos (\lambda)$ & 0 & 0 & 0 \\
\hline 22 & 0 & 45.349 & $\begin{array}{c}\text { Polar } \\
\mathrm{S}\end{array}$ & W & $\begin{array}{l}\left(90^{\circ}-\lambda\right) \\
\text { Below } \mathrm{N}\end{array}$ & $-\sin (\lambda)$ & 0 & $-\cos (\lambda)$ & 0 & 0 \\
\hline 23 & 270 & 45.349 & $\begin{array}{c}\text { Polar } \\
\mathrm{S}\end{array}$ & $\begin{array}{l}\left(90^{\circ}-\lambda\right) \\
\text { Below N }\end{array}$ & $\mathrm{E}$ & $-\sin (\lambda)$ & $-\cos (\lambda)$ & 0 & 0 & 0 \\
\hline 24 & 180 & 45.349 & $\begin{array}{c}\text { Polar } \\
\text { S }\end{array}$ & $\mathrm{E}$ & $\begin{array}{c}\left(90^{\circ}-\lambda\right) \\
\text { Above S }\end{array}$ & $-\sin (\lambda)$ & 0 & $\cos (\lambda)$ & 0 & 0 \\
\hline
\end{tabular}


Table 4.2: Definition of Gyroscope Axes Positions for an 11 Position Test

\begin{tabular}{|c|c|c||c|c|c||c|c|c||c|c|}
\hline \multicolumn{3}{|c|}{ Position } & \multicolumn{3}{c||}{ Direction of Axes } & \multicolumn{3}{c||}{ Acceleration Along } & \multicolumn{2}{c|}{ Earth Rate Input } \\
\hline \# & Az. & Tilt & Spin & X & Y & Spin & X & Y & X & Y \\
\hline \hline 1 & 90 & 180 & Up & N & W & 1 & 0 & 0 & $\omega_{e} \cos (\lambda)$ & 0 \\
\hline 2 & 0 & 180 & Up & W & S & 1 & 0 & 0 & 0 & $-\omega_{e} \cos (\lambda)$ \\
\hline 3 & 270 & 180 & Up & S & E & 1 & 0 & 0 & $-\omega_{e} \cos (\lambda)$ & 0 \\
\hline 4 & 180 & 180 & Up & E & N & 1 & 0 & 0 & 0 & $\omega_{e} \cos (\lambda)$ \\
\hline 5 & 180 & 270 & N & E & Dn & 0 & 0 & -1 & 0 & $-\omega_{e} \sin (\lambda)$ \\
\hline 6 & 270 & 270 & N & Up & E & 0 & 1 & 0 & $\omega_{e} \sin (\lambda)$ & 0 \\
\hline 7 & 0 & 270 & N & W & Up & 0 & 0 & 1 & 0 & $\omega_{e} \sin (\lambda)$ \\
\hline 8 & 90 & 270 & N & Dn & W & 0 & -1 & 0 & $-\omega_{e} \sin (\lambda)$ & 0 \\
\hline 9 & 225 & 270 & N & $45^{\circ}$ & $45^{\circ}$ & 0 & $\cos \left(\frac{\pi}{4}\right)$ & $-\cos \left(\frac{\pi}{4}\right)$ & $\omega_{e} \sin (\lambda)$ & $-\omega_{e} \sin (\lambda)$ \\
& & & & Above E & Below E & & & & $\cos \left(\frac{\pi}{4}\right)$ & $\cos \left(\frac{\pi}{4}\right)$ \\
\hline 10 & 45 & 270 & N & $45^{\circ}$ & $45^{\circ}$ & 0 & $-\cos \left(\frac{\pi}{4}\right)$ & $\cos \left(\frac{\pi}{4}\right)$ & $-\omega_{e} \sin (\lambda)$ & $\omega_{e} \sin (\lambda)$ \\
& & & & Below W & Above W & & & & $\cos \left(\frac{\pi}{4}\right)$ & $\cos \left(\frac{\pi}{4}\right)$ \\
\hline 11 & 90 & 0 & Down & S & W & -1 & 0 & 0 & $-\omega_{e} \sin (\lambda)$ & 0 \\
\hline
\end{tabular}




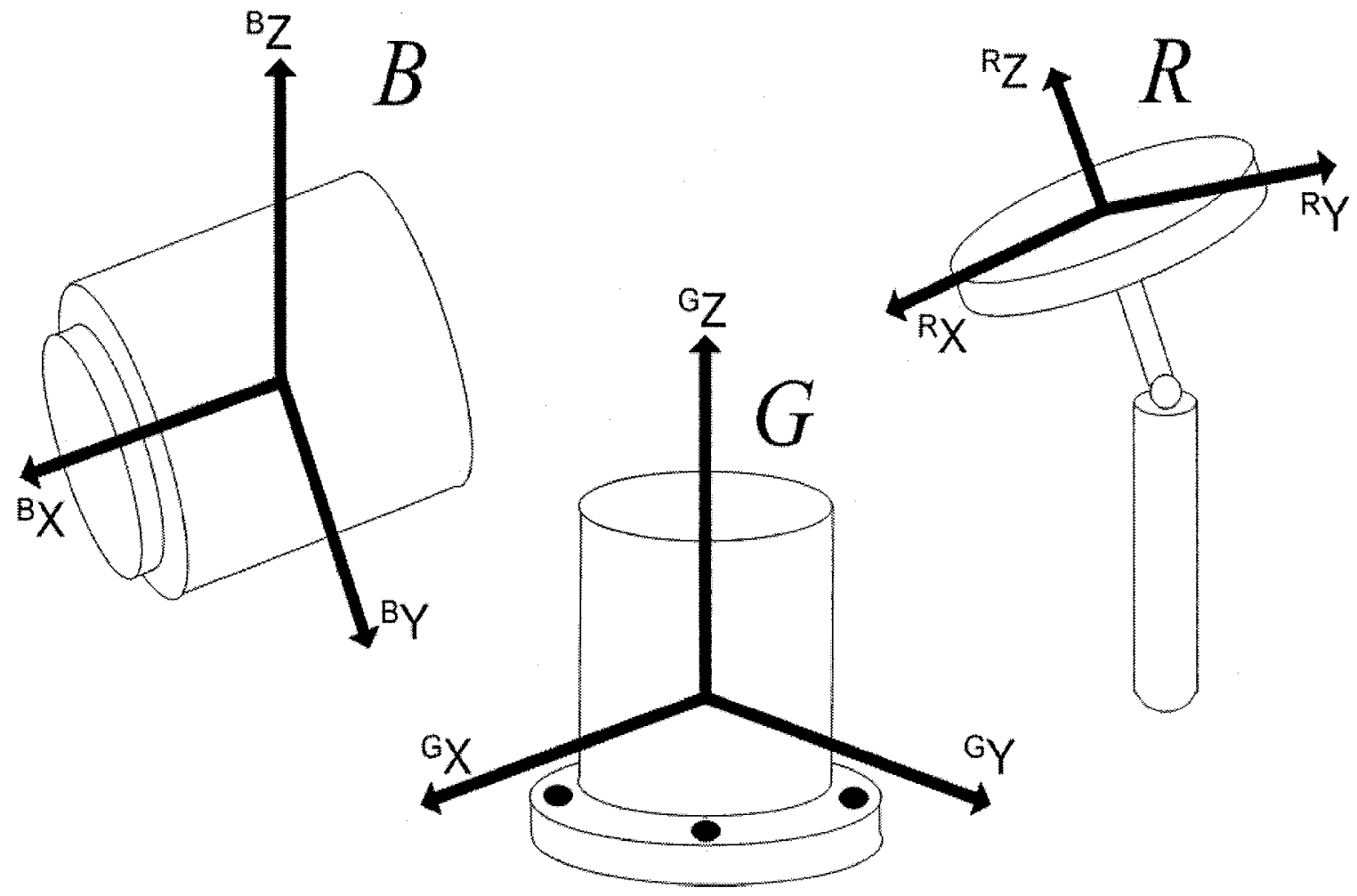

Figure 4.4: Definition of the GyroWheel ${ }^{\mathrm{TM}}$ Main Frames of Reference. 


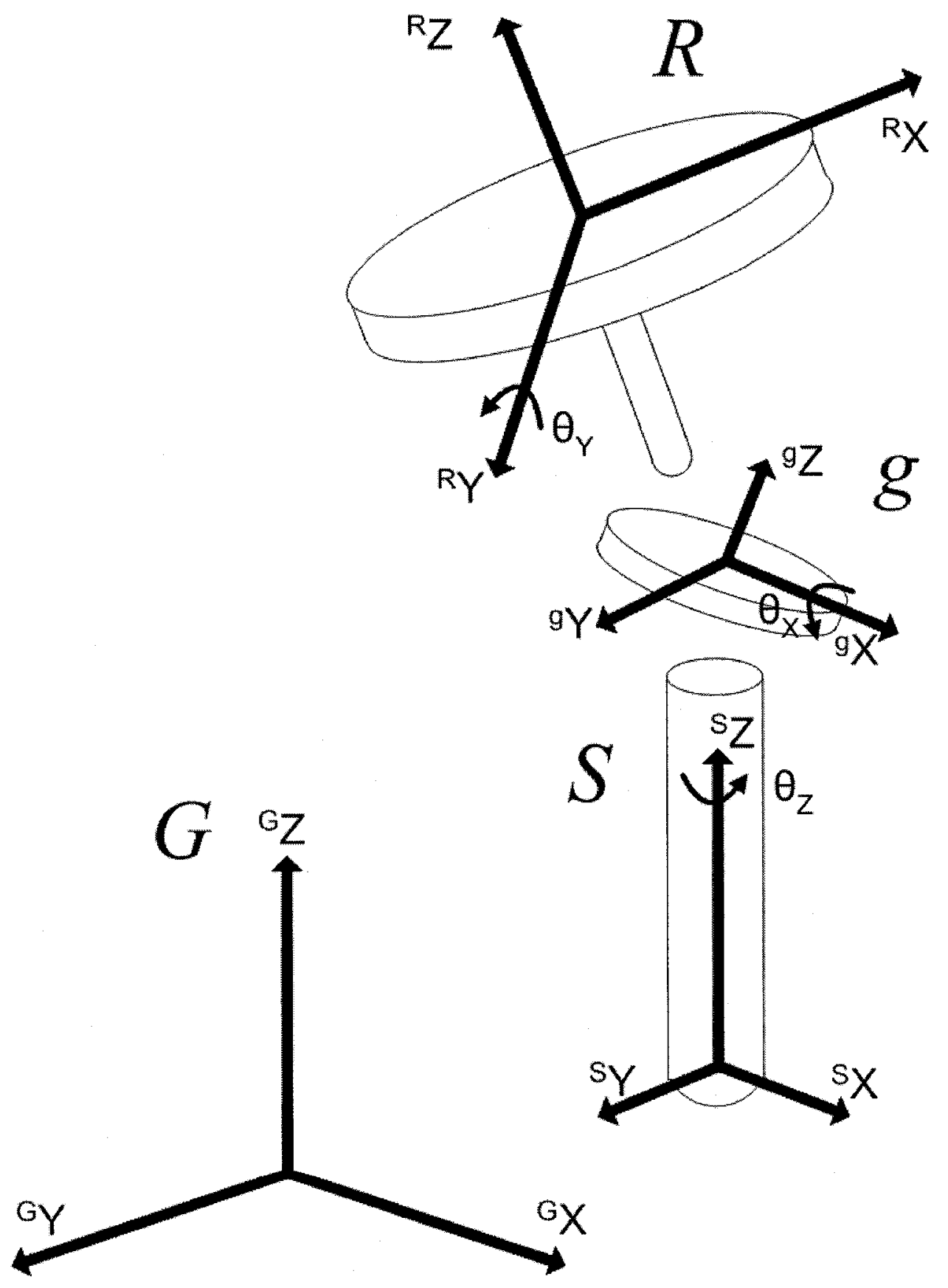

Figure 4.5: Definition of the GyroWheel ${ }^{\mathrm{TM}}$ Additional Frames of Reference. 
in the spacecraft coordinate system B, would be equal to the sum of the Spacecraft Momentum, the Wheel Momentum due to the spacecraft motion, and the Wheel Momentum due to relative motion or

$$
{ }^{B} \boldsymbol{H}_{\mathrm{tot}}={ }^{B} \boldsymbol{H}_{\mathrm{s} / \mathrm{c}}+{ }^{B} \boldsymbol{H}_{\mathrm{wheel}, \mathrm{s} / \mathrm{c}}+{ }^{B} \boldsymbol{h}
$$

Utilizing the principle of Impulse-Momentum, the equations of motion could then be defined since the derivative of the total momentum with respect to time would equal the sum of all external torques

$$
\frac{\mathrm{d}\left({ }^{B} \boldsymbol{H}_{\mathrm{tot}}\right)}{\mathrm{d} t}=\sum_{i} \boldsymbol{T}_{\mathrm{ext}, i}
$$

In reality though, the GyroWheel ${ }^{\mathrm{TM}}$ is not a double-gimbal momentum wheel. This is illustrated in Figure 4.5 above, showing the three main moving bodies of the GyroWheel ${ }^{\mathrm{TM}}$ : the shaft (including the motor rotor); the gimbal attached to the shaft through four flexures as shown in Figure 4.6; and the rotor attached to the gimbal through four more flexures. This model is presented in detail by Ower [17] and is called the Three-Body, Non-Linear Dynamic Model. It is discussed very briefly below.

\section{Three-Body Model}

The total kinetic energy, neglecting the kinetic energy stored in the flexure bending motion due to a lumped properties assumption, is given by

$$
T=\frac{1}{2}\left(\boldsymbol{\omega}_{\mathrm{s}}^{T} \mathbf{I}_{\mathrm{s}} \boldsymbol{\omega}_{\mathrm{s}}+\boldsymbol{\omega}_{\mathrm{g}}^{T} \mathbf{l}_{\mathrm{g}} \boldsymbol{\omega}_{\mathrm{g}}+\boldsymbol{\omega}_{\mathrm{r}}^{T} \mathbf{I}_{\mathrm{r}} \boldsymbol{\omega}_{\mathrm{r}}\right)
$$

The potential energy is the strain energy stored in the flexure deformation dependent on the two pairs of flexure stiffness $\left(K_{x}, K_{y}\right)$ and the two gimbal angles $\left(\theta_{x}, \theta_{y}\right)$

$$
V=\frac{1}{2}\left(K_{x} \theta_{x}^{2}+K_{y} \theta_{y}^{2}\right)
$$

This assumes that the gimbal assembly functions as a Hooke's Joint. 


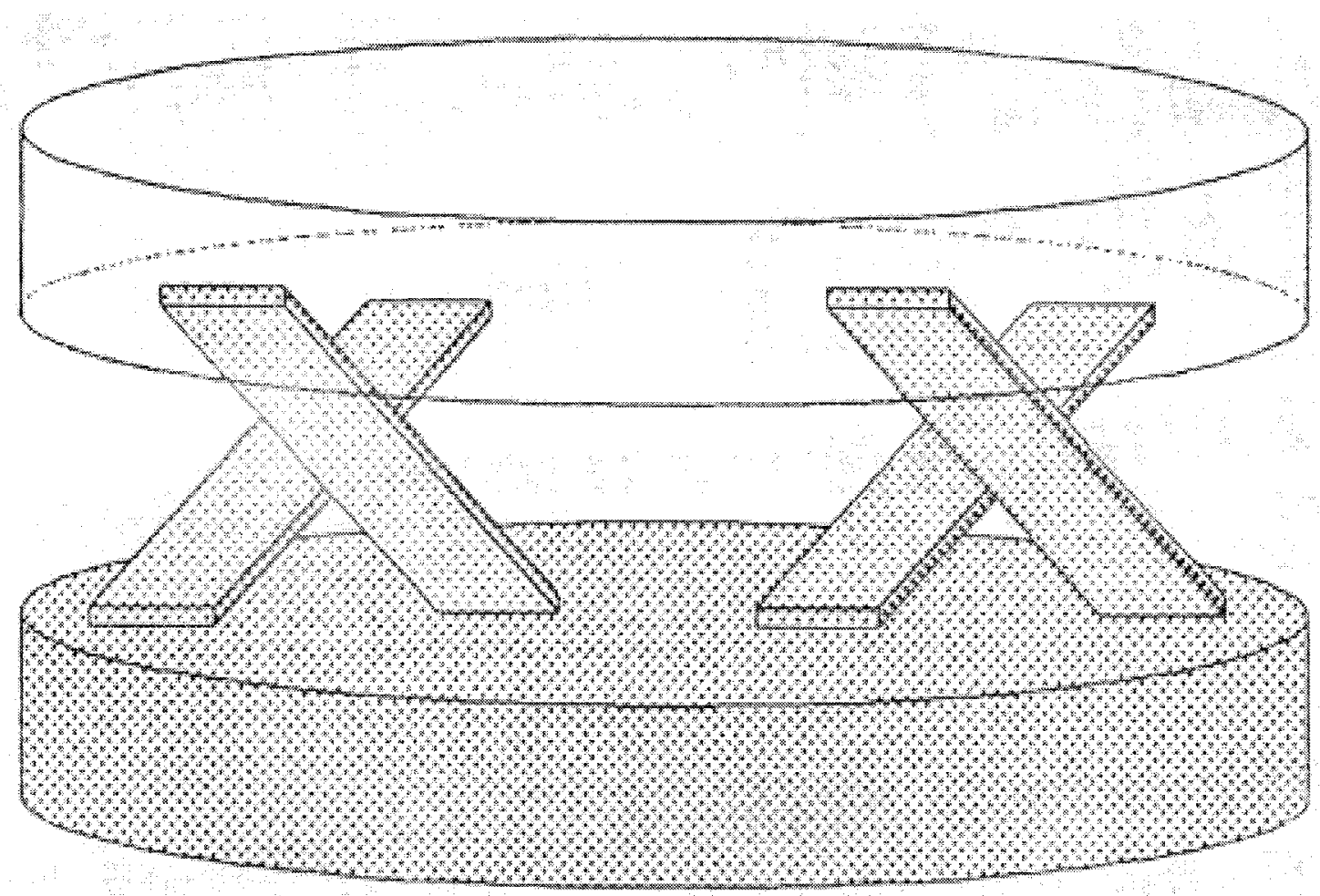

Figure 4.6: Sketch of GyroWheel ${ }^{\mathrm{TM}}$ Gimbal: Utilized in the Explanation of the GyroWheel $^{\text {TM }}$ Three-Body, Non-Linear Dynamic Model. 


\section{Equations of Motion}

The Lagrangian of the system is defined as:

$$
L=T-V
$$

The three equations of motion can now be determined by applying Lagrange's equations

$$
\frac{\mathrm{d}}{\mathrm{d} t}\left(\frac{\partial L}{\partial \dot{q}_{i}}\right)-\frac{\partial L}{\partial q_{i}}=Q_{i}
$$

where $Q_{i}$ is a generalized torque acting about a gimbal axis $i$. Each generalized torques consists of a generalized control torque and in the case of the flexure pivot axes, a structural damping torque. $C_{x}$ and $C_{y}$ will be the damping coefficients of the two crossed-flexure pivot pairs. The complete derivation of the full three-body non-linear dynamic model is given by Ower [17]. The resulting model has the form

$$
\mathrm{M}(\boldsymbol{q}) \ddot{q}+\mathrm{C} \dot{q}+\mathrm{K} \boldsymbol{q}=T_{c}+F_{\mathrm{nl}}\left(\boldsymbol{q}, \dot{q}, \omega_{c}, \dot{\omega}_{c}\right)
$$

where $\boldsymbol{q}$ represents the gimbal angles and shaft rotation angle, and $\mathbf{T}_{\mathrm{c}}$ is the vector of control torques

$$
\boldsymbol{q}=\left\{\begin{array}{c}
\theta_{x} \\
\theta_{y} \\
\theta_{z}
\end{array}\right\}, \boldsymbol{T}_{c}=\left\{\begin{array}{c}
T_{c X} \\
T_{c Y} \\
T_{c Z}
\end{array}\right\}
$$

$\mathrm{C}$ and $\mathrm{K}$ are the damping and stiffness matrices representative of the flexure damping and spring stiffness coefficients

$$
\begin{gathered}
\mathrm{C}=\left[\begin{array}{ccc}
C_{x} & 0 & 0 \\
0 & C_{y} & 0 \\
0 & 0 & 0
\end{array}\right], \mathrm{K}=\left[\begin{array}{ccc}
K_{x} & 0 & 0 \\
0 & K_{y} & 0 \\
0 & 0 & 0
\end{array}\right] \\
\boldsymbol{F}_{\mathrm{n} 1}\left(\boldsymbol{q}, \dot{\boldsymbol{q}}, \boldsymbol{\omega}_{c}, \dot{\boldsymbol{\omega}}_{\boldsymbol{c}}\right)=\left\{\begin{array}{c}
f_{\mathrm{n} 11}\left(\boldsymbol{q}, \dot{\boldsymbol{q}}, \boldsymbol{\omega}_{c}, \dot{\boldsymbol{\omega}}_{\boldsymbol{c}}\right) \\
f_{\mathrm{n} 12}\left(\boldsymbol{q}, \dot{\boldsymbol{q}}, \boldsymbol{\omega}_{c}, \dot{\boldsymbol{\omega}}_{\boldsymbol{c}}\right) \\
f_{\mathrm{n} 13}\left(\boldsymbol{q}, \dot{\boldsymbol{q}}, \boldsymbol{\omega}_{\boldsymbol{c}}, \dot{\boldsymbol{\omega}}_{\boldsymbol{c}}\right)
\end{array}\right\}
\end{gathered}
$$


are a set of three non-linear functions in $\boldsymbol{q}, \dot{\boldsymbol{q}}, \boldsymbol{\omega}_{c}, \dot{\boldsymbol{\omega}}_{c}$ that are not presented here, but can be found in Ower [17] Appendix A, pages 401 - 403.

\subsubsection{Actuator Model}

The actuators consist of four coils located in the $\pm \mathrm{x}$ and $\pm \mathrm{y}$ directions, as discussed in Chapter 3.1.6, and one motor to spin the rotor. The coils interact with a magnetic field generated by permanent magnet rings attached to the rotor. Each coil is considered to generate a wrench (force vectors $\left(\boldsymbol{F}_{ \pm i}\right)$ and moment vectors $\left(\boldsymbol{M}_{ \pm i}\right)$ ) about the origin of the coil located at a distance $r_{ \pm i}$ from the origin of the gimbal frame, g. The forces and moments are proportional to the current in the coil such that

$$
\begin{gathered}
\boldsymbol{F}_{ \pm i}=\boldsymbol{k} \mathbf{1}_{ \pm i} i_{ \pm i} \\
\boldsymbol{M}_{ \pm i}=\boldsymbol{k} \mathbf{2}_{ \pm i} i_{ \pm i}
\end{gathered}
$$

Since the scale factor for each coil is dependent on the magnetic flux through the coil, and since the field is moving with the rotor, the scale factor vectors $k 1$ and $\mathbf{k 2}$ for each coil are tilt dependent.

In general, $\boldsymbol{T}_{c}$ can be defined as the sum of the moments around the reference point $\mathbf{O}_{\text {coil }}$ as shown in Figure 4.7 from de Carufel [9]. De Carufel's derivation is simplified with the following assumptions:

- each coil is considered to produce only a force and not a moment $(\boldsymbol{k} 2$ 's are zero and therefore $M_{ \pm i}=0$ )

- the B field for opposite coils and the coils themselves are parallel and symmetric $\left(k 1_{+x}=-k 1_{-x} ; k 1_{+y}=k 1_{-y} ; r_{x}=-r_{-x} ;\right.$ and $\left.r_{y}=-r_{-y}\right)$

- the coils are connected in series such that the current in each coil is the same $\left(i_{+x}=i_{-x}\right.$ and $\left.i_{+y}=i_{-y}\right)$ 


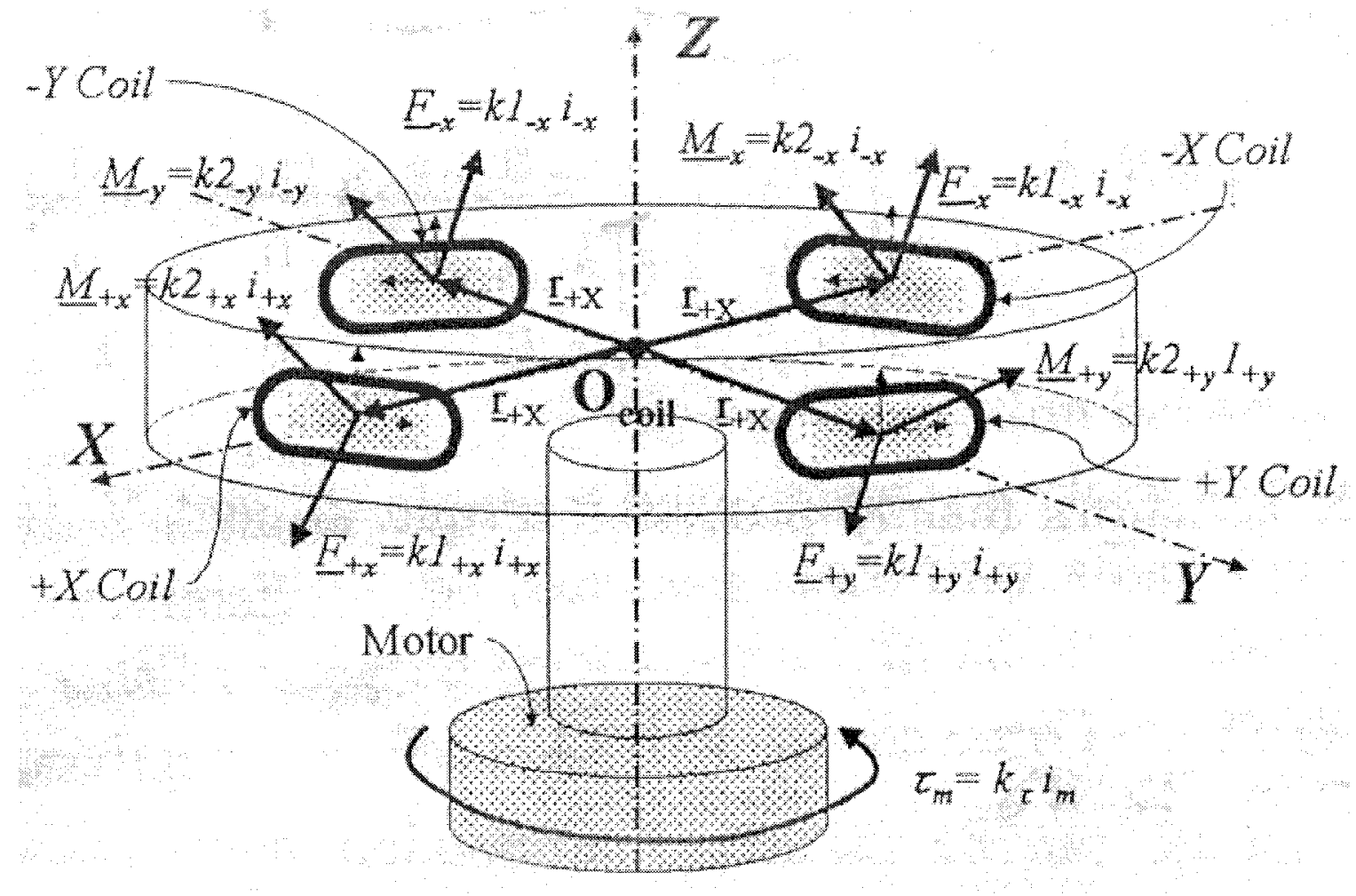

Figure 4.7: GyroWheel ${ }^{\text {TM }}$ Actuator Model 
The mapping between the control currents and $\boldsymbol{T}_{\boldsymbol{c}}$ then reduces to

$$
\boldsymbol{T}_{\boldsymbol{c}}=\left[\begin{array}{ccc}
0 & k_{\mathrm{scal}, y} & 0 \\
-k_{\mathrm{scale}, x} & 0 & 0 \\
0 & 0 & k_{\tau}
\end{array}\right]\left\{\begin{array}{c}
i_{X} \\
i_{Y} \\
i_{\text {motor }}
\end{array}\right\}
$$

demonstrating that the $\pm x$ actuators mainly produce torques about the $\mathrm{y}$ axis and the $\pm y$ actuators produce torques about the $\mathrm{x}$ axis.

\subsubsection{Linearizing About an Operating Point}

The three-body nonlinear model can be linearized around an operating point in the gimbal coordinates as shown by Ower [17]. The linearization occurs about the nominal operating condition of zero spin axis tilt with a constant spin rate, $\omega_{z}$. Additionally there is no angular velocity about the $\mathrm{X}$ and $\mathrm{Y}$ axes and no angular accelerations about any axis.

$$
\begin{aligned}
& \dot{\theta}_{z}=\omega_{z} \\
& \theta_{x}=\theta_{y}=0 \\
& \dot{\theta}_{x}=\dot{\theta}_{y}=0 \\
& \ddot{\theta}_{x}=\ddot{\theta}_{y}=\ddot{\theta}_{z}=0
\end{aligned}
$$

Additionally, the following assumptions were made to simplify the model without losing any accuracy:

- the rotor transverse inertias were assumed to be equal and set to the value $I_{r t}$. Similarly, the gimbal transverse inertias were assumed to be equal and set to the value $I_{g t}$

$$
\begin{aligned}
& I_{r x}=I_{r y}=I_{r t} \\
& I_{g x}=I_{g y}=I_{g t}
\end{aligned}
$$


- the spin inertias for the rotor and gimbal are re-assigned the variable names $I_{r s}$ and $I_{g s}$ to more clearly distinguish between $\operatorname{spin}_{s}$ and transverse ${ }_{t}$

- the gimbal damping factors $C_{x}$ and $C_{y}$ were set equal and assigned the common gimbal damping term $c_{g}$

The model must then be expressed in terms of the output being controlled (tilt angles with respect to the case) and the control input (torques from the torquer coils). Therefore the equations of motion must be transformed into expressions in terms of the GyroWheel ${ }^{\mathrm{TM}}$ Frame, G. This is achieved by examining the relationship between the gimbal angles $\theta_{x}$ and $\theta_{y}$ and the case tilt angles where

$$
\left\{\begin{array}{c}
\theta_{x} \\
\theta_{y}
\end{array}\right\}=\left[\begin{array}{cc}
\cos \left(\theta_{z}\right) & \sin \left(\theta_{z}\right) \\
-\sin \left(\theta_{z}\right) & \cos \left(\theta_{z}\right)
\end{array}\right]\left\{\begin{array}{c}
\phi_{x} \\
\phi_{y}
\end{array}\right\}
$$

Therefore the linearized dynamic equation of motion in the $\mathrm{x}$-axis expressed in the GyroWheel ${ }^{\mathrm{TM}}$ Frame is given by

$$
\begin{array}{r}
{\left[I_{r t}+\frac{I_{g t}}{2}\left(1+\cos \left(2 \omega_{z} t\right)\right)\right] \ddot{\phi}_{x}+\left(c_{g}+I_{g t} \sin \left(2 \omega_{z} t\right)\right) \omega_{z} \dot{\phi}_{x}} \\
+\left[\left(\frac{K_{x}+K_{y}}{2}-J \omega_{z}^{2}\right)+\left(\frac{K_{x}-K_{y}}{2}-J \omega_{z}^{2}\right) \cos \left(2 \omega_{z} t\right)\right] \phi_{x} \\
-\frac{I_{g t}}{2} \sin \left(2 \omega_{z} t\right) \ddot{\phi}_{y}+\left[I_{r s}+I_{g t} \omega_{z}\left(1-\cos \left(2 \omega_{z} t\right)\right)\right] \dot{\phi}_{y} \\
+\left(2 c_{g} \omega_{z}+\left(\frac{K_{x}-K_{y}}{2}-J \omega_{z}^{2}\right) \sin \left(2 \omega_{z} t\right)\right) \phi_{y} \\
+\left[I_{r t}+\frac{I_{g t}}{2}\left(1+\cos \left(2 \omega_{z} t\right)\right)\right] \dot{\omega}_{c X}-\frac{I_{g t}}{2} \sin \left(2 \omega_{z} t\right) \dot{\omega}_{c Y} \\
-\frac{I_{g t}}{2} \omega_{z} \sin \left(2 \omega_{z} t\right) \omega_{c X}+\left(I_{r s}+\frac{I_{g s}}{2}\left(1+\cos \left(2 \omega_{z} t\right)\right)\right) \omega_{z} \omega_{c Y} \\
=T_{c X}
\end{array}
$$


and in the $y$-axis is given by

$$
\begin{array}{r}
{\left[I_{r t}+\frac{I_{g t}}{2}\left(1+\cos \left(2 \omega_{z} t\right)\right)\right] \ddot{\phi}_{y}+\left(c_{g}+I_{g t} \omega_{z} \sin \left(2 \omega_{z} t\right)\right) \dot{\phi}_{x}} \\
+\left[\left(\frac{K_{x}+K_{y}}{2}-J \omega_{z}^{2}\right)+\left(\frac{K_{x}-K_{y}}{2}-J \omega_{z}^{2}\right) \cos \left(2 \omega_{z} t\right)\right] \phi_{y} \\
-\frac{I_{g t}}{2} \sin \left(2 \omega_{z} t\right) \ddot{\phi}_{x}+\left[I_{r s}+I_{g t}\left(1-\cos \left(2 \omega_{z} t\right)\right)\right] \omega_{z} \dot{\phi}_{x} \\
-\left(2 c_{g} \omega_{z}+\left(\frac{K_{x}-K_{y}}{2}-J \omega_{z}^{2}\right) \sin \left(2 \omega_{z} t\right)\right) \phi_{x} \\
+\left[I_{r t}+\frac{I_{g t}}{2}\left(1+\cos \left(2 \omega_{z} t\right)\right)\right] \dot{\omega}_{c Y}-\frac{I_{g t}}{2} \sin \left(2 \omega_{z} t\right) \dot{\omega}_{c X} \\
-\frac{I_{g t}}{2} \omega_{z} \sin \left(2 \omega_{z} t\right) \omega_{c Y}+\left(I_{r s}+\frac{I_{g s}}{2}\left(1+\cos \left(2 \omega_{z} t\right)\right)\right) \omega_{z} \omega_{c X} \\
=T_{c Y}
\end{array}
$$

In the above two equations, the term $J$ is defined as

$$
J=I_{g t}-\frac{I_{g s}}{2}
$$

The $2 \omega_{z}$ terms are the result of the transformation between the tilt angles expressed in the Gimbal Frame and those in the GyroWheel ${ }^{\mathrm{TM}}$ base frame. The effect of these cyclic terms is very small on the dynamics of the system since they affect the terms related to the gimbal inertia only, which is usually small compared to the rotor inertia. In addition, the effect of those terms can be reduced by notch filtering the measured signals used for rate sensing at $2 \omega_{z}$ or by averaging over one or two rotational periods. It is therefore common practice to include these as small $2 \omega_{z}$ cyclic disturbances and neglect them in the design of the tilt control. This the reduces the dynamic equations to

$$
\begin{aligned}
\left(I_{r t}+\right. & \left.\frac{I_{g t}}{2}\right) \ddot{\phi}_{x}+c_{g} \dot{\phi}_{x}+\left(\frac{K_{x}+K_{y}}{2}-J \omega_{z}^{2}\right) \phi_{x}+\left(I_{r s}+I_{g t}\right) \omega_{z} \dot{\phi}_{y} \\
& +2 c_{g} \omega_{z} \phi_{y}+\left(I_{r t}+\frac{I_{g t}}{2}\right) \dot{\omega}_{c X}+\left(I_{r s}+\frac{I_{g s}}{2}\right) \omega_{z} \omega_{c Y}=T_{c X}
\end{aligned}
$$


and

$$
\begin{array}{r}
\left(I_{r t}+\frac{I_{g t}}{2}\right) \ddot{\phi}_{y}+c_{g} \dot{\phi}_{y}+\left(\frac{K_{x}+K_{y}}{2}-J \omega_{z}^{2}\right) \phi_{y}+\left(I_{r s}+I_{g t}\right) \omega_{z} \dot{\phi}_{x} \\
-2 c_{g} \omega_{z} \phi_{x}+\left(I_{r t}+\frac{I_{g t}}{2}\right) \dot{\omega}_{c Y}+\left(I_{r s}+\frac{I_{g s}}{2}\right) \omega_{z} \omega_{c X}=T_{c Y}
\end{array}
$$

\subsection{GyroWheel ${ }^{\text {TM }}$ Rate Sensing}

\subsubsection{GyroWheel ${ }^{\mathrm{TM}}$ Flight Rate Sensing Equation}

Substituting the values for $\boldsymbol{T}_{\boldsymbol{c}}$ from Equation 4.45 into Equations 4.52 and 4.53 as well as isolating the spacecraft motion to one side of the equation produces

$$
\begin{aligned}
\left(I_{r t}+\right. & \left.\frac{I_{g t}}{2}\right) \dot{\omega}_{c X}+\left(I_{r s}+\frac{I_{g s}}{2}\right) \omega_{z} \omega_{c Y}=k_{\text {scale }, y} i_{y}-\left(I_{r t}+\frac{I_{g t}}{2}\right) \ddot{\phi}_{x} \\
& -c_{g} \dot{\phi}_{x}-\left(\frac{K_{x}+K_{y}}{2}-J \omega_{z}^{2}\right) \phi_{x}-\left(I_{r s}+I_{g t}\right) \omega_{z} \dot{\phi}_{y}-2 c_{g} \omega_{z} \phi_{y}
\end{aligned}
$$

and

$$
\begin{array}{r}
\left(I_{r t}+\frac{I_{g t}}{2}\right) \dot{\omega}_{c Y}-\left(I_{r s}+\frac{I_{g s}}{2}\right) \omega_{z} \omega_{c X}=-k_{\text {scale }, x} i_{x}-\left(I_{r t}+\frac{I_{g t}}{2}\right) \ddot{\phi}_{y} \\
-c_{g} \dot{\phi}_{y}-\left(\frac{K_{x}+K_{y}}{2}-J \omega_{z}^{2}\right) \phi_{y}+\left(I_{r s}+I_{g t}\right) \omega_{z} \dot{\phi}_{x}+2 c_{g} \omega_{z} \phi_{x}
\end{array}
$$

In order to simplify the notation lets define

$$
\begin{aligned}
K_{\mathrm{dyn}} & =\frac{K_{x}+K_{y}}{2}-J \omega_{z}^{2} \\
h_{s} & =\left(I_{r s}+\frac{I_{g s}}{2}\right) \omega_{z}
\end{aligned}
$$

Additionally it can be assumed that the angular acceleration is negligible, eliminating the $\dot{\omega}_{c}$ and $\ddot{\phi}$ terms. Equations 4.55 and 4.54 respectively can now be written as

$$
\omega_{c X}=\frac{1}{h_{s}}\left[k_{\text {scale }, x} i_{x}+c_{g} \dot{\phi}_{y}+K_{\mathrm{dyn}} \phi_{y}-\left(I_{r s}+I_{g t}\right) \omega_{z} \dot{\phi}_{x}-2 c_{g} \omega_{z} \phi_{x}\right]
$$




$$
\omega_{c Y}=\frac{1}{h_{s}}\left[k_{\text {scale }, y} i_{y}-c_{g} \dot{\phi}_{x}-K_{\mathrm{dyn}} \phi_{x}-\left(I_{r s}+I_{g t}\right) \omega_{z} \dot{\phi}_{y}-2 c_{g} \omega_{z} \phi_{y}\right]
$$

Given that $\left(I_{r s}+\frac{I_{g s}}{2}\right)$ is constant, this value will be absorbed into the coefficients for each term in the above equations. This will leave only $\omega_{z}$ in the denominator on the right hand side of the above equations. It will then be multiplied out on the two $\phi$ terms containing $\omega_{z}$. Further, lets define $I_{t, x}$ and $I_{t, y}$ as the axis specific transverse inertia terms to be applied to the $\dot{\phi}$ terms; $c_{g, x}$ and $c_{g, y}$ as the axis specific damping terms to be applied to the $\phi$ and $\dot{\phi}$ terms respectively; and $K_{\mathrm{dyn}, x}$ and $K_{\mathrm{dyn}, y}$ as the axis specific dynamic stiffness terms to be applied to the $\phi$ terms. These axis specific terms are defined for temperature calibration purposes so that differences between the $\mathrm{x}$ and $\mathrm{y}$ axes can be taken into account in calibration. The above equations can now be written as

$$
\begin{aligned}
& \omega_{c X}=\frac{1}{\omega_{z}}\left[k_{\text {scale }, x} i_{x}+c_{g, y} \dot{\phi}_{y}+K_{\mathrm{dyn}, y} \phi_{y}\right]-I_{t, x} \dot{\phi}_{x}-2 c_{g, x} \phi_{x} \\
& \omega_{c Y}=\frac{1}{\omega_{z}}\left[k_{\text {scale }, y} \dot{y}_{y}-c_{g, x} \dot{\phi}_{x}-K_{\mathrm{dyn}, x} \phi_{x}\right]-I_{t, y} \dot{\phi}_{y}-2 c_{g, y} \phi_{y}
\end{aligned}
$$

Equations 4.60 and 4.61 now define the spacecraft angular velocity about the $\mathrm{X}$ and $\mathrm{Y}$ axis in terms measured directly by the GyroWheel ${ }^{\mathrm{TM}}$ as discussed in Section 3.2.4

\subsubsection{GyroWheel ${ }^{\mathrm{TM}}$ Error Terms}

While it is desirable to have the rate input axes aligned with the torquer axes, manufacturing realities often result in the rate input axes being misaligned from the torquer axes of the gyro. These misalignments must be factored into the analysis to obtain accurate rate sensing. As shown in Figure 4.8, $\alpha$ is the torquer misalignment term for the $\mathrm{x}$ rate input axis and therefore $\alpha+\beta$ is the torquer misalignment term for the y rate input axis. Remembering that $i_{x}$ is defined as the current in the torque coils physically located on the GyroWheel ${ }^{\mathrm{TM}} \mathrm{x}$ case axis and therefore

$$
X_{\text {torquer }}=k_{\text {scale }, y} i_{y} \text { and } Y_{\text {torquer }}=k_{\text {scale }, x} i_{x}
$$




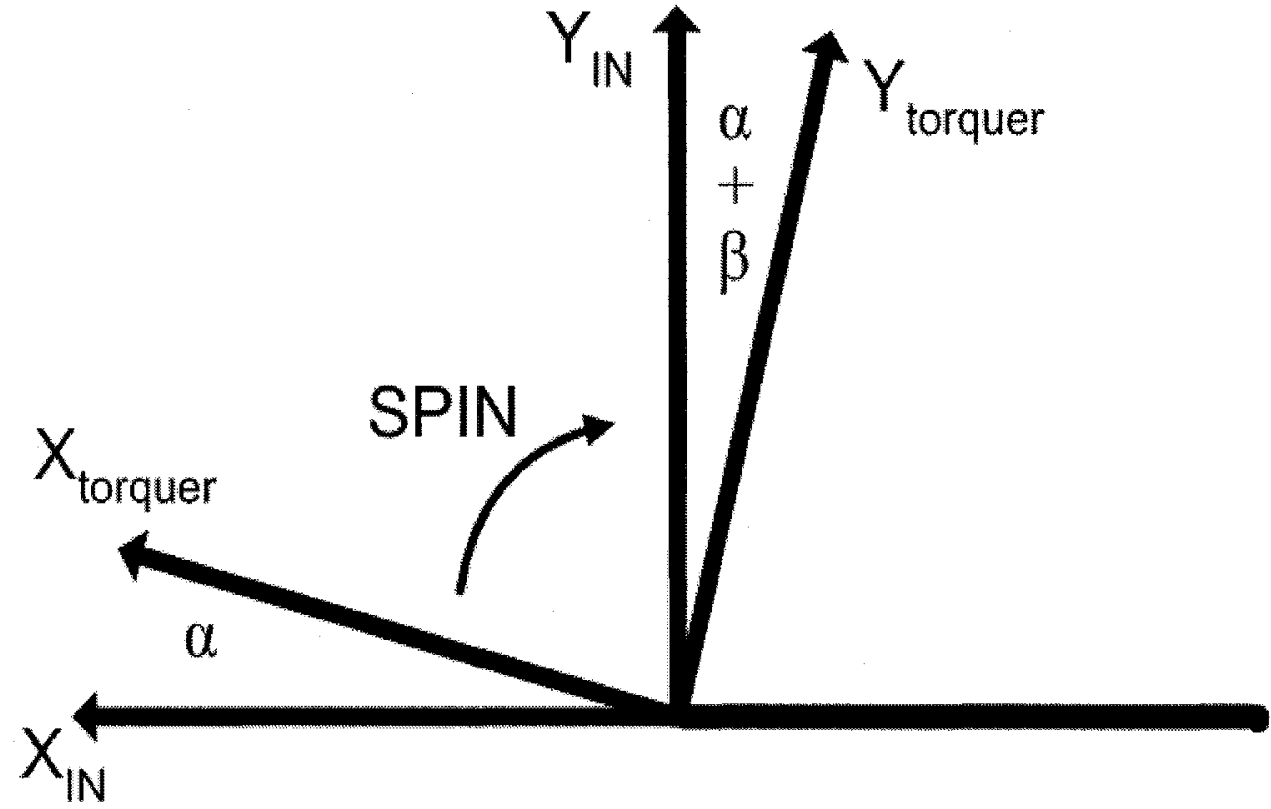

Figure 4.8: Coordinates of GyroWheel ${ }^{\mathrm{TM}}$ Rate Input and Torquer Axes Misalignments 
In order to take into account the misalignment angles when determining the spacecraft angular velocity, $k_{\text {scale }, x} i_{x}$ and $k_{\text {scale, } y} i_{y}$ as defined in Equations 4.60 and 4.61 should be defined as

$$
\begin{aligned}
& k_{\text {scale }, x} i_{x}=k_{x} i_{x} \cos (\alpha+\beta)+k_{y} i_{y} \sin (\alpha) \\
& k_{\text {scale }, y} i_{y}=k_{y} i_{y} \cos (\alpha)-k_{x} i_{x} \sin (\alpha+\beta)
\end{aligned}
$$

where $k_{x}$ and $k_{y}$ are the experimentally determined scale factors that will be calibrated for changes in temperature.

Additionally all gyros have an absolute bias term, $B_{x}$ or $B_{y}$, as discussed in Section 4.1.5. Incorporating these three pure error related terms, $\alpha, \beta$, and $B$, Equations 4.60 and 4.61 now become

$$
\begin{aligned}
& \omega_{c X}=\frac{1}{\omega_{z}}\left[k_{x} i_{x} \cos (\alpha+\beta)+k_{y} i_{y} \sin (\alpha)+c_{g, y} \dot{\phi}_{y}+K_{\mathrm{dyn}, y} \phi_{y}\right]-I_{t, x} \dot{\phi}_{x}-2 c_{g, x} \phi_{x}-B_{x} \\
& \omega_{c Y}=\frac{1}{\omega_{z}}\left[k_{y} i_{y} \cos (\alpha)-k_{x} i_{x} \sin (\alpha+\beta)-c_{g, x} \dot{\phi}_{x}-K_{\mathrm{dyn}, x} \phi_{x}\right]-I_{t, y} \dot{\phi}_{y}-2 c_{g, y} \phi_{y}-B_{y}
\end{aligned}
$$

\subsubsection{GyroWheel ${ }^{\mathrm{TM}}$ Ground Calibration}

The equations above cannot be used in a ground calibration due to the presence of gravitational acceleration in a ground calibration situation. The presence of gravity acts as an acceleration, aggravating various error terms that are normally ignored in micro-gravity, where there are no substantial gravitational accelerations. These errors are discussed in Section 4.1.3 and are mainly due to rotor and gimbal mass unbalance as well as non-uniformity of the flexure stiffness and damping. They are also shown in the general rebalance Equations 4.29 and 4.30. Incorporating these additional error terms into Equations 4.65 and 4.66, the ground test rate sensing 
equations become

$$
\begin{aligned}
\omega_{c X} & =\frac{1}{\omega_{z}}\left[k_{x} i_{x} \cos (\alpha+\beta)+k_{y} i_{y} \sin (\alpha)+c_{g, y} \dot{\phi}_{y}+K_{\mathrm{dyn}, y} \phi_{y}\right] \\
& -I_{t, x} \dot{\phi}_{x}-2 c_{g, x} \phi_{x}-B_{x}-D(x)_{X} a_{X}-D(x)_{Y} a_{Y}-D(x)_{Z} a_{Z} \\
& -D(x)_{X X} a_{X}^{2}-D(x)_{Z Z} a_{Z}^{2}-D(x)_{X Y} a_{X} a_{Y}-D(x)_{X} a_{X} a_{Z}-D(x)_{Y Z} a_{Y} a_{Z} \\
\omega_{c Y} & =\frac{1}{\omega_{z}}\left[k_{y} i_{y} \cos (\alpha)-k_{x} i_{x} \sin (\alpha+\beta)-c_{g, x} \dot{\phi}_{x}-K_{\mathrm{dyn}, x} \phi_{x}\right] \\
& -I_{t, y} \dot{\phi}_{y}-2 c_{g, y} \phi_{y}-B_{y}-D(y)_{Y} a_{Y}-D(y)_{X} a_{X}-D(y)_{Z} a_{Z} \\
& -D(y)_{Y Y} a_{Y}^{2}-D(y)_{Z Z} a_{Z}^{2}-D(y)_{Y X} a_{X} a_{Y}-D(y)_{X Z} a_{X} a_{Z}-D(y)_{Y Z} a_{Y} a_{Z}
\end{aligned}
$$

where $a_{x}, a_{y}$ and $a_{z}$ are acceleration components of the gravity vector in the tilted rotor frame; $D(x)_{i}, i \in\{x, y, z\}$ and $D(y)_{i}, i \in\{x, y, z\}$ are experimentally determined calibration errors representing drift terms sensitive to acceleration due to gravity, known as g-sensitive terms; and $D(x)_{i j}, i j \in\{x x, y y, z z, x y, x z, y z\}$ and $D(y)_{i j}, i j \in\{x x, y y, z z, x y, x z, y z\}$ are experimentally determined calibration errors representing drift terms sensitive to the square of acceleration due to gravity, known as g-squared sensitive terms. As discussed at Equations 4.29 and 4.30, the $D(x)_{Y Y} a_{Y}^{2}$ and $D(y)_{X X} a_{X}^{2}$ terms are not included as they cannot be determined utilizing static $1 \mathrm{~g}$ testing and are therefore assumed to be negligible during such tests.

Equations 4.67 and 4.68 represent a total of 28 terms that potentially require calibration. Often with DTGs, it has been the experience that many of the drift terms are negligible. This is particularly true of the g-squared sensitive terms. However, DTGs typically have a low rotor inertia compared to the GyroWheel ${ }^{\mathrm{TM}}$, since it requires a significantly larger rotor in order to additionally function as a momentum actuator. The GyroWheel ${ }^{\mathrm{TM}}$ rotor mass is approximately twenty times that of a typical DTG. Therefore, no term can be assumed to be negligible without proper testing.

Testing was first conducted to determine which, if any, of the error terms can be deemed negligible. This then reduced the number of error terms to be calibrated 
in the above equations, which reduced the number of positions required when conducting multi-positional testing as discussed in Section 4.2.1. Once the number of positions required for multi-positional testing was determined, the testing for temperature calibration was conducted. The specific tests and test equipment utilized are discussed in the next Chapter. 


\section{Chapter 5}

\section{GyroWheel $^{\text {TM }}$ Testing}

This Chapter describes the equipment utilized in the collection of the data required in the calibration of the GyroWheel ${ }^{\mathrm{TM}}$, as well as the specific testing carried out with reference to the motivation behind the testing and end product to be determined. Clean Room procedures, to a clean room standard of Class 100,000, were enforced during the entire test program.

\subsection{Equipment Utilized}

Testing was carried out using highly specialized equipment and facilities at the inertial navigation department of Defence Research and Development Centre Ottawa at Shirley's Bay. This facility is one of a small number of similar facilities of its kind in Canada and is specially equipped to do motion testing of inertial instruments. The laboratory has been developed so that data collection has been automated wherever possible. 


\subsubsection{Contraves Motion Table}

The Contravese Motion Table is the base of all GyroWheel TM testing in that all other test equipment was built around this large highly specialized piece of equipment. It is a two axis model 57CD motion simulator manufactured by Contraves Goerz and is controlled by a 30H MPACS level III command system (Modular Precision Angular Control System). From Hartl [12], the motion table itself is capable of positioning accuracy of \pm 1 arcsecond, with a resolution capability of 0.36 arcseconds $\left(0.0001^{\circ}\right)$

The table has been surveyed in place so that the principle axes of the motion table align with the North-South, East-West geographic cardinal directions. The trunnion axis (elevation) is aligned with the Earth's East-West axis, and the table spin axis (azimuth) is perpendicular to the elevation axis. This alignment was done at the time of installation into the facility. However, because of the accuracy requirements of this thesis, a precise table alignment was required. Precise alignment of the table and the mounted GyroWheel ${ }^{\mathrm{TM}}$ is necessary to determine what fraction of earth rate is being sensed in a given table orientation. As describe by Hartl [12] in his Section 4.4.3.1, an auto collimating theodolite and a North seeking gyro, known as GAX are utilized to determine a north line bearing better than 10 arcseconds of certainty in an iterative process. Once the site line to North is determined, the motion table can be aligned. Further, before the GyroWheel ${ }^{T M}$ is mounted on the table top, it is necessary to ensure the motion table is leveled. This is accomplished by adjusting the three leveling screws that the motion table rests on. Spirit levels are utilized in the accomplishment of the leveling, moving from coarse 200 arcsecond levels, to 10 and then 3 arcsecond levels for fine adjustment.

A specially designed fixture, built by DRDC-Ottawa, is used to attach and position the GyroWheel ${ }^{\mathrm{TM}}$ on top of the Contraves Motion Table. The table top mounting plate is a circular 19" diameter disc made from 6061-T6 Aluminum, that is mounted on the table's azimuth axis. The plate was machined to tolerances of 
0.1 thousandths of an inch in all critical dimensions. Furthermore, a chromate conversion coat was applied to the surface in order to make the surface resistant to corrosion in order to maintain its flatness. Spirit levels are again used to assess the levelness of the mounting plate. Since the azimuth axis can rotate, there is also a possibility of coning of the azimuth axis. The mounting plate can also be shimmed, and the tension in the mounting screws can be adjusted in order to correct for this coning. The cooling plate and the GyroWheel ${ }^{\mathrm{TM}}$ are then mounted and leveled on the mounting plate. The mounting plate was fitted with the electrical heaters utilized to control the temperature of the GyroWheel ${ }^{\mathrm{TM}}$ and the cooling plate was fitted with the cooling tubes as discussed in the next section.

\subsubsection{Temperature Control}

During GyroWheel ${ }^{\mathrm{TM}}$ testing the temperature was manually controlled to ensure that the GyroWheel ${ }^{\mathrm{TM}}$ did not get outside of the ranges for testing of $9^{\circ} \mathrm{C}$ to $55^{\circ} \mathrm{C}$. $T_{h, x}$, the Housing Temperature $\mathrm{x}$ value in degrees Celsius, as discussed in Section 3.2.4 was utilized as the setpoint value for temperature control. The temperature of the mounting plate was provided utilizing a Fluke thermometer.

\section{Heating System}

Electrical heaters were used to raise the temperature of the mounting plate attached to the motion table. It was determined experimentally that if the heater control variac was put at $15 \%$ then the mounting plate would not go above 45 degrees Celsius. A mounting plate temperature of $45^{\circ} \mathrm{C}$ corresponds to a GyroWheel ${ }^{\mathrm{TM}}$ $T_{h, x}$ temperature that would not go above $55^{\circ} \mathrm{C}$, the operating limit allowed for this testing. The variac was adjusted to a higher value on initial heating from a cold state in order to more quickly enact a temperature change. The variac setting was reduced as the temperature increased. 


\section{Cooling System}

A circular 18" diameter cooling plate made by DRDC-Ottawa from 6061-T6 Aluminum was mounted on the base plate. The cooling plate was machined so that copper cooling tubes could be embedded in the cooling plate as per Figure 5.1. A methanol based Endocal Refrigerated Circulation Bath was connected to the input and output ports on the cooling plate. It was determined experimentally that if the methanol circulation bath was set at $-15^{\circ} \mathrm{C}$ then the cooling plate would not go below -20 degrees Celsius. A cooling plate temperature of $-20^{\circ} \mathrm{C}$ corresponds to a GyroWheel ${ }^{\mathrm{TM}} T_{h, x}$ temperature that would not go below $9^{\circ} \mathrm{C}$ degrees Celsius, the operating limit allowed for this testing. The methanol circulation bath was set to a lower value on initial cooling from a hot state in order to more quickly enact a temperature change. The methanol circulation bath temperature setting was increased as the temperature decreased.

\subsubsection{Vacuum Chamber}

The GyroWheel ${ }^{\text {TM }}$ was tested under vacuum conditions to simulate the space environment and to detcrmine the true flight calibration relationships and performance parameters. In order to accomplish this, an environmental test housing was built from 6061-T6 Aluminum by DRDC-Ottawa and mounted to the cooling plate and around the GyroWheel ${ }^{T M}$. A schematic of the vacuum chamber is shown in Figure 5.2. The vacuum chamber can be evacuated to below 1 Torr.

The overall setup for mounting the GyroWheel ${ }^{\mathrm{TM}}$ on the motion table is shown in Figure 5.3 including the base plate, cooling plate, and the vacuum chamber.

\subsubsection{Data Monitoring and Collection}

The Electrical Ground Support Equipment (EGSE) provides a user interface for issuing control commands, viewing telemetry, and uploading or downloading GyroWheel ${ }^{T M}$ 

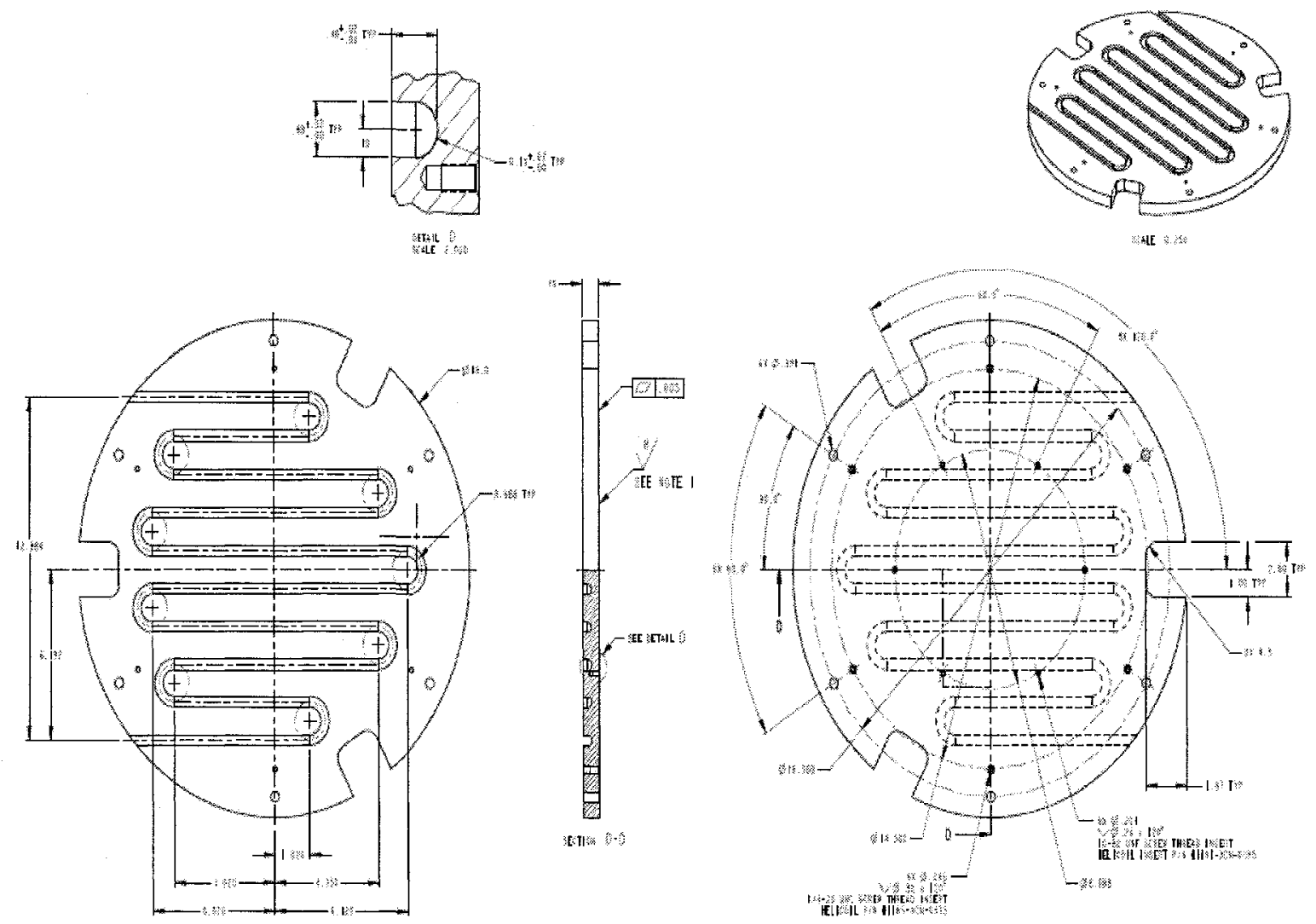

Figure 5.1: Engineering Drawing of the GyroWheel ${ }^{\mathrm{TM}}$ Cooling Plate 


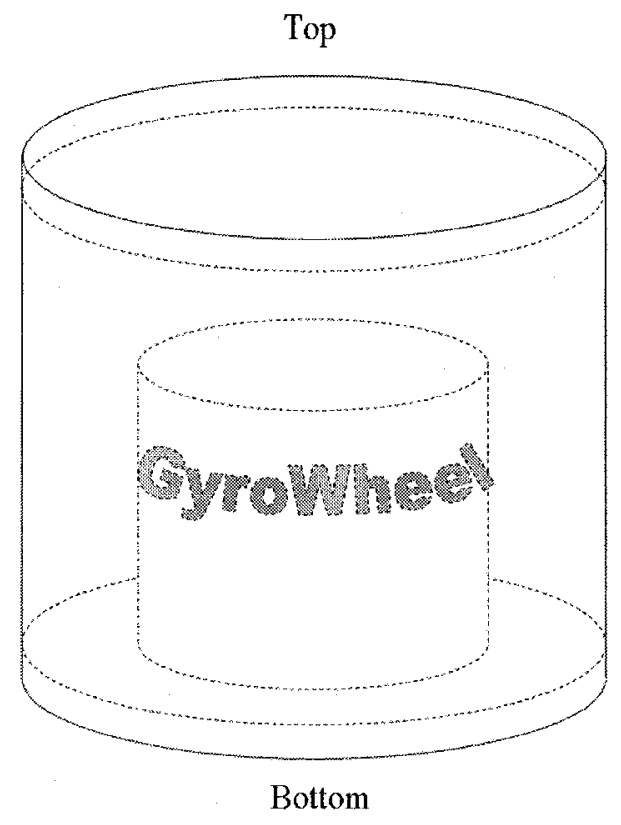

Figure 5.2: Schematic Representation of the GyroWheel ${ }^{\mathrm{TM}}$ Vacuum Chamber

data and memory. The precise measured values provided by the GyroWheel ${ }^{\mathrm{TM}}$ as discussed in Section 3.2.4 are provided through the EGSE Computer. The EGSE also provides the user interface for applying GyroWheel diagnostics and the interface for raw electrical signals. The GyroWheel ${ }^{\mathrm{TM}}$ and EGSE are connected through the vacuum chamber via a wiring harness.

Due to cost limitations and the fact that the power level for the electrical heaters would induce noise on the signals being measured from the GyroWheel ${ }^{\mathrm{TM}}$, it was not possible for the signals and flows to be commutated through the contravese motion table slip ring assembly or hydraulics commutation. Therefore, an important consideration with the testing was ensuring that the electrical cables, vacuum chamber hoses, cooling tubes, and the heater control cables did not get tangled when moving the gyroscope between the different multi-position tests. For these reasons testing required nearly constant supervision. A photo of the entire testing apparatus 


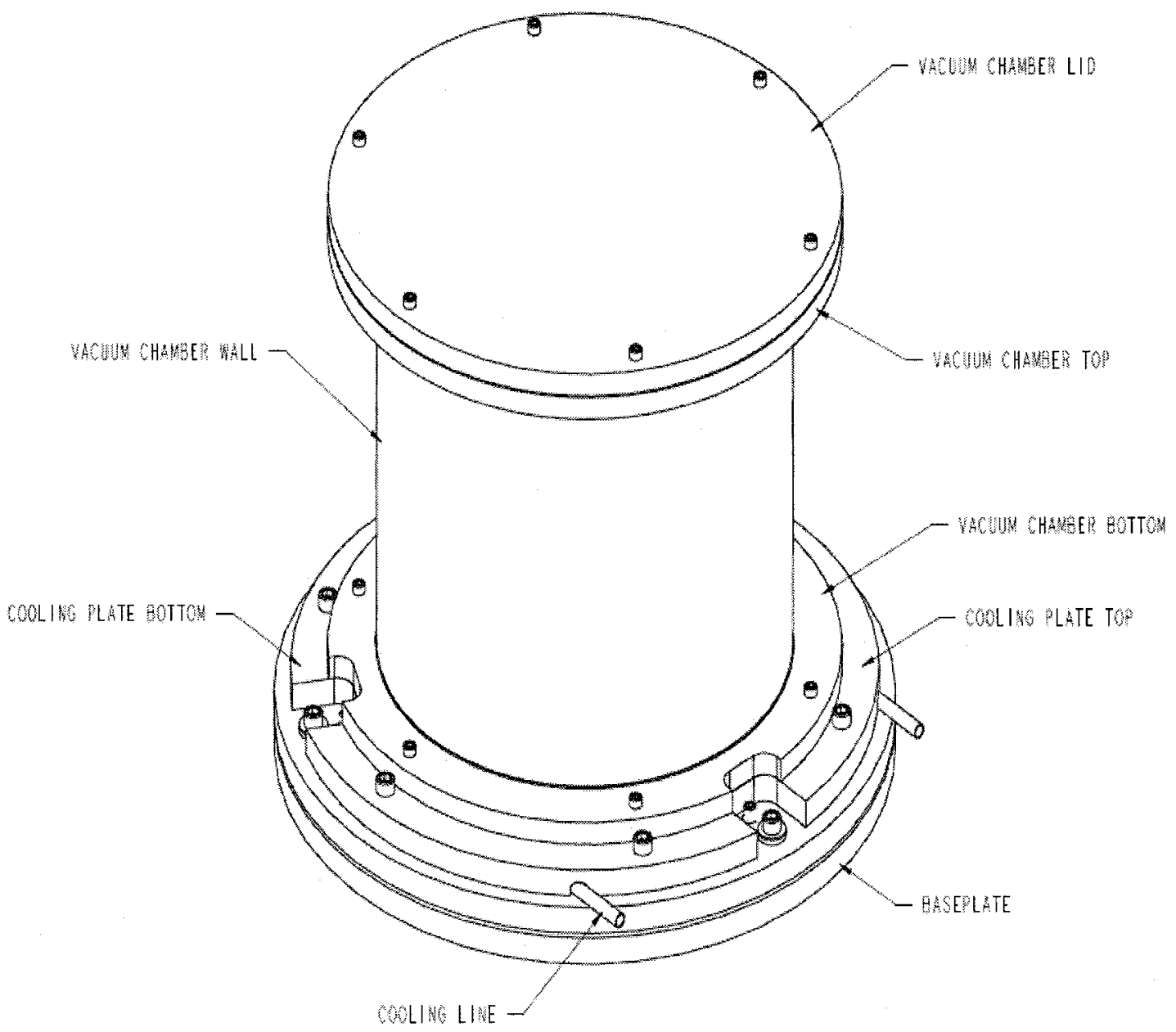

Figure 5.3: Engineering Drawing of the GyroWheel ${ }^{\mathrm{TM}}$ Test Equipment Mounted to the Motion Table 
setup is included as Figure 5.4.

For testing, operator intervention was required to: attach and remove the methanol cooling hoses from the cooling plate; change the coolant temperature settings and heater power settings; change the position of the motion table; change the GyroWheel ${ }^{T M}$ trajectory file, rotor tilt, or wheel speed; monitoring and adjusting the tempcrature control units to maintain the GyroWheel ${ }^{\mathrm{TM}}$ housing temperature within operational limits $\left(9^{\circ} \mathrm{C}\right.$ to $\left.55^{\circ} \mathrm{C}\right)$; maintaining the test log; and changing the automatic data recording parameters and file names.

\subsection{Description of Tests}

\subsubsection{Turn On - Turn On Repeatability}

Turn on - turn on repeatability tests were performed by repeating multi-position tests. The GyroWheel ${ }^{\mathrm{TM}}$ is turned on and allowed to come to thermal equilibrium prior to commencing the test. Once the full multi-position test is complete, the calibration coefficients are calculated from the test data. The unit is then turned off and allowed to cool down. The same multi-position test is then repeated and the resulting calibration values are compared.

Schultz [19] conducted three turn on - turn on 11 position tests at tuned conditions and determined that the turn on - turn on bias repeatability of the GyroWheel TM is $\pm 0.46 \mathrm{deg} / \mathrm{hr}$ on a bias magnitude of $92.79 \mathrm{deg} / \mathrm{hr}$. This represents $\pm 0.50 \%$.

\subsubsection{Drift Test}

Drift testing is accomplished by orienting the GyroWheel ${ }^{\mathrm{TM}}$ to a predetermined table position. Temperature, motion table position, rotor tilt, and wheel speed are kept constant. The long term drift and noise in the measured torque coil currents indicate the accuracy obtainable for determining position through integrating the 


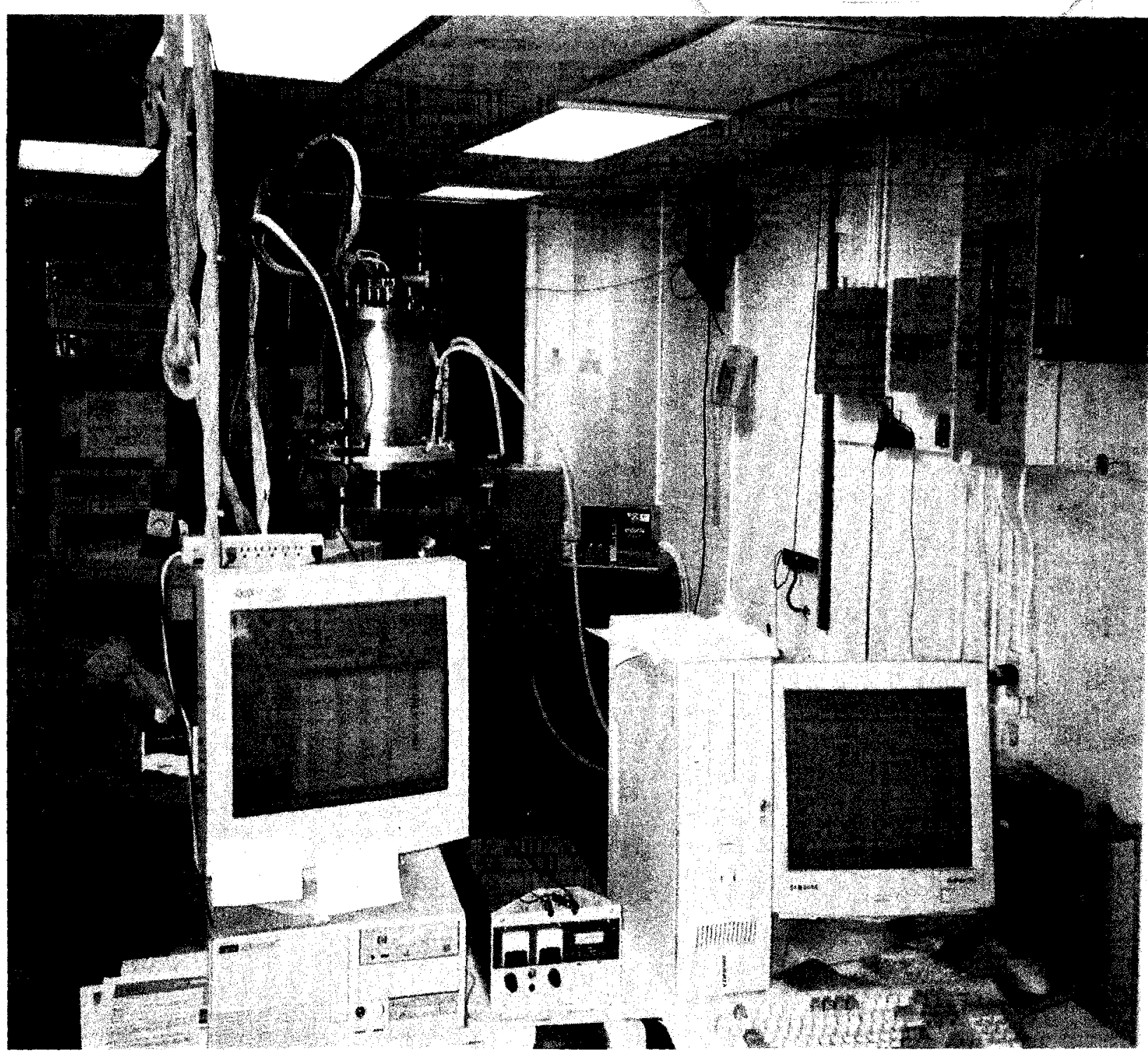

Figure 5.4: Picture of the GyroWheel ${ }^{\mathrm{TM}}$ Test Equipment in the Inertial Navigation Laboratory 
measured rate. Schultz [19] performed drift testing for periods up to 75 hours at tuned conditions. He determined that the worst case random drift rate was found to be $0.316 \mathrm{deg} / \mathrm{hr}(1 \sigma)$ at a rate input of $10.5 \mathrm{deg} / \mathrm{hr}$.

\subsubsection{Multi-Position Testing}

As stated in Section 4.2.1 discussing multi-position testing, it is often not required to determine all of the drift parameters in the general equation since some of the error terms may be insignificant, especially in high quality gyroscopes. As discussed by Schultz [19] initial testing of the GyroWheel ${ }^{T M}$ showed that the g-sensitive and some $\mathrm{g}^{2}$-sensitive drift terms were not negligible. These drift terms ranged from $0.06 \%$ to $50 \%$ of the magnitude of the bias drift. During flight operations, the importance of the $\mathrm{g}$-sensitive and $\mathrm{g}^{2}$-sensitive terms is reduced, however the number of these drift terms that are deemed negligible in ground calibration determines the number of positions required when conducting multi-positional testing and has a large effect on the test plan time lines.

Multi-position testing was completed for a 15 position test at tuned conditions. The data was then analyzed utilizing the singular value decomposition method explained in Section 6.1. Analyses of the data from a 15 position test solves for the following error terms from Equations 4.67 and 4.68:

$$
\begin{array}{r}
\alpha, \beta, B_{x}, B_{y}, D(x)_{X}, D(y)_{Y}, D(x)_{Y}, D(y)_{X}, \\
D(x)_{Z}, D(y)_{Z}, D(x)_{X X}, D(y)_{Y Y}, D(x)_{Z Z}, D(y)_{Z Z}, \\
D(x)_{X Y}, D(y)_{Y X}, D(x)_{X Z}, D(y)_{X Z}, D(x)_{Y Z}, D(y)_{Y Z}
\end{array}
$$

The 15 position test data was then analyzed as if two 13 position tests were completed, utilizing both positions $(1,2,3,4,5,6,7,9,10,11,12,15)$ as well as positions $(1,2,3,4,5,6,7,8,9,10,13,14,15)$. Further, the data was analyzed as if only an 11 position test $(1,2,3,4,5,6,7,8,9,10,15)$, and finally an 8 position test $(1,2,3,4,5,6,7,8)$ were 
completed. The calibration coefficients resulting from these analyses as well as the standard deviations of the coefficients is presented at Appendix A.

From the different multi-position data, the standard deviations of the calibration coefficients improve from the 15 position test, to the 13 position test. Additionally, for both different 13 position tests, the coefficients are the same to 4 decimal places. When comparing the results as if an 11 position test was conducted with the results as if either of the 13 positions tests were conducted, the calculated values of the coefficients are exactly the same to four decimal places. However, changes start occuring in the calibration coefficients when comparing the results as if an 8 position test was conducted instead of an 11 position test. Based on this data, it is deemed that the following error terms are negligible for GyroWheel ${ }^{\mathrm{TM}}$ calibration: $D(x)_{X Z}, D(y)_{X Z}, D(x)_{Y Z}, D(y)_{Y Z}$. Equations 4.67 and 4.68 then become finally

$$
\begin{aligned}
\omega_{c X} & =\frac{1}{\omega_{z}}\left[k_{x} i_{x} \cos (\alpha+\beta)+k_{y} i_{y} \sin (\alpha)+c_{g, y} \dot{\phi}_{y}+K_{\mathrm{dyn}, y} \phi_{y}\right] \\
& -I_{t, x} \dot{\phi}_{x}-2 c_{g, x} \phi_{x}-B_{x}-D(x)_{X} a_{X}-D(x)_{Y} a_{Y}-D(x)_{Z} a_{Z} \\
& -D(x)_{X X} a_{X}^{2}-D(x)_{Z Z} a_{Z}^{2}-D(x)_{X Y} a_{X} a_{Y} \\
\omega_{c Y} & =\frac{1}{\omega_{z}}\left[k_{y} i_{y} \cos (\alpha)-k_{x} i_{x} \sin (\alpha+\beta)-c_{g, x} \dot{\phi}_{x}-K_{\mathrm{dyn}, x} \phi_{x}\right] \\
& -I_{t, y} \dot{\phi}_{y}-2 c_{g, y} \phi_{y}-B_{y}-D(y)_{Y} a_{Y}-D(y)_{X} a_{X}-D(y)_{Z} a_{Z} \\
& -D(y)_{Y Y} a_{Y}^{2}-D(y)_{Z Z} a_{Z}^{2}-D(y)_{Y X} a_{X} a_{Y}
\end{aligned}
$$

Furthermore, 11 position multi-position testing is required in order to properly calibrate the GyroWheel ${ }^{\mathrm{TM}}$. The positions required for 11 position testing are presented earlier in Table 4.2. All of the following described tests were performed in each of the 11 positions required to determine the above calibration coefficients of the GyroWheel ${ }^{\mathrm{TM}}$. 


\subsubsection{Temperatures During Testing}

The temperature testing comprised the largest portion of the testing completed on the GyroWheel ${ }^{T M}$. The GyroWheel ${ }^{T M}$ housing temperature had to be maintained between the temperature range of $9^{\circ} \mathrm{C}$ to $55^{\circ} \mathrm{C}$, and when discussing the temperature testing the term cold will refer to approximately $10^{\circ} \mathrm{C}$ and hot will refer to approximately $55^{\circ} \mathrm{C}$. Constant temperature testing was conducted at one of three temperatures: room or ambient temperature; hot; and cold, with the exact temperature being provided by the GyroWheel ${ }^{\mathrm{TM}}$ EGSE system.

\section{Temperature Sweep Testing}

It was also necessary to see how the calibration was affected by changes in temperature throughout the temperature range. Therefore, the data collected during this test allowed for the determination of the temperature calibration of the rate-sensing equations of the GyroWhee ${ }^{\mathrm{TM}}$ over the operating temperature range. Motion table position, wheel speed and rotor tilt are kept constant, but are not necessarily at tuned conditions. In each motion table position, the temperature is swept from hotto-cold-to-hot over an eight hour period. Because of a compressed testing schedule due to the limited time available with the GyroWhee ${ }^{\mathrm{TM}}$ unit prior to satellite integration, near constant operator intervention was required in order to maximize the speed of the temperature changes without going outside of the GyroWheel ${ }^{\mathrm{TM}}$ housing temperature operational limits. This constant operator intervention allowed for the shortening of the test length from sixteen hours (minimal supervision) to eight hours for each position. Table 5.1 shows the sequence for the temperature sweep testing. 
Table 5.1: Eight hour period temperature sweep time-line.

\begin{tabular}{|c|c|l|l|}
\hline Step & Time [hrs] & \multicolumn{1}{|c|}{ Physical Action Required } & Supervision Time Required \\
\hline 1 & $0: 00$ & $\begin{array}{l}\text { Heater off; methanol cooling flow } \\
\text { started with methanol reservoir } \\
\text { setpoint at }-60^{\circ} \mathrm{C}\end{array}$ & $\begin{array}{l}15 \text { minutes at the start of } \\
\text { the step to attach cooling } \\
\text { hoses, adjust settings, and } \\
\text { change the table position }\end{array}$ \\
\hline 2 & $1: 00$ & $\begin{array}{l}\text { Methanol reservoir temperature } \\
\text { intermittent adjustment from } \\
-60^{\circ} \mathrm{C} \text { to }-15^{\circ} \mathrm{C} \text { over the next } 3 \\
\text { hours at } 20 \text { minute intervals }\end{array}$ & $\begin{array}{l}3 \text { hours (the whole step) to } \\
\text { perform temperature } \\
\text { adjustments }\end{array}$ \\
\hline 3 & $4: 00$ & $\begin{array}{l}\text { Heater started at } 40 \% \text { setting; } \\
\text { Methanol cooling system stopped }\end{array}$ & $\begin{array}{l}15 \text { minutes at the start of } \\
\text { the step to remove cooling } \\
\text { hoses and adjust settings }\end{array}$ \\
\hline 4 & $5: 40$ & $\begin{array}{l}\text { Heater intermittent adjustment } \\
\text { from } 40 \% \text { to } 15 \% \text { over the next } \\
2.33 \text { hours }\end{array}$ & $\begin{array}{l}\text { 2.33 hours (the whole step) } \\
\text { to perform heater power } \\
\text { adjustments }\end{array}$ \\
\hline 5 & $8: 00$ & $\begin{array}{l}\text { (same as step 1); repeat the cycle } \\
\text { at a new wheel/table position }\end{array}$ & (same as step 1) \\
(see 1) & & \multicolumn{2}{|l}{} \\
\hline
\end{tabular}




\subsubsection{Tilt Sweep Testing}

The data collected during this test can be used to determine the rotor tilt rate related calibration constants of the GyroWheel ${ }^{\mathrm{TM}}$. Temperature, motion table position, and wheel speed are kept constant. The GyroWheel ${ }^{\mathrm{TM}}$ is set to follow a commanded tilt, profile and the test duration is typically one hour. Operator intervention is required at the beginning and end of the test to stop and start the commanded tilt profile. In this test, as temperature is at a steady state value, it requires no operator intervention for control.

\subsubsection{Wheel Speed During Testing}

Testing was conducted at one of three wheel speeds:

- Nominal Tuned Speed $=152.89 \mathrm{rad} / \mathrm{s}$

- $-15 \%$ Nominal Tuned Speed $=129.96 \mathrm{rad} / \mathrm{s}$

- $+15 \%$ Nominal Tuned Speed $=175.82 \mathrm{rad} / \mathrm{s}$

\subsubsection{Combined Temperature and Tilt Sweep Testing}

In order to make more efficient use of the data collection time available with the GyroWheel $^{\text {TM }}$ it was decided to vary the tilt conditions of the GyroWheel ${ }^{\text {TM }}$ during the temperature sweeps. The temperature profile run was as follows:

- 1 hour at $T_{h, x}=52-53^{\circ} \mathrm{C}$ - "hot dwell"

- 3 hours cooling to $T_{h, x}=9-10^{\circ} \mathrm{C}$

- 1 hour at $T_{h, x}=9-10^{\circ} \mathrm{C}$ - "cold dwell"

- 3 hours heating to $T_{h, x}=52-53^{\circ} \mathrm{C}$ 
The tilt conditions were changed every 11 minutes with 16 minutes spent on the first "null" tilt condition in order to maintain a one hour timing for the tilt sweep trajectory. The tilt sweep trajectory is therefore run eight times during each temperature sweep. An example of the tilt sweep trajectory utilizing a tilt angle of three degrees is shown below:

- 16 minutes at $\phi_{x}=0 \mathrm{deg} ; \phi_{y}=0 \mathrm{deg}$

- 11 minutes at $\phi_{x}=3 \mathrm{deg} ; \phi_{y}=0 \mathrm{deg}$

- 11 minutes at $\phi_{x}=0 \mathrm{deg} ; \phi_{y}=3 \mathrm{deg}$

- 11 minutes at $\phi_{x}=-3 \mathrm{deg} ; \phi_{y}=0 \mathrm{deg}$

- 11 minutes at $\phi_{x}=0 \mathrm{deg} ; \phi_{y}=-3 \mathrm{deg}$

Both the motion table position and the wheel speed are kept constant during each combined temperature and tilt sweep test. A combined temperature and tilt sweep test was completed for each motion table position for 11 position testing at each of the three wheel speeds mentioned above. This resulted in 264 hours of testing for each tilt angle tested. With respect to temperature, this testing will provide a graph broken into 11 minute segments for each tilt position (16 minutes for null) as the temperature changes, as well as a constant temperature for each of the five tilt positions at both the hot and cold temperature levels. Separate testing was completed at the ambient or room temperature value. In the time frame allotted with the GyroWheel ${ }^{\mathrm{TM}}$ for testing, combined temperature and tilt sweep testing was completed for tilts of $1.5 \mathrm{deg}$ and $3 \mathrm{deg}$, resulting in over 528 hours of testing.

\subsection{Data Extraction and Clean-up}

The data files collected during testing were separated into distinct sections depending on which variable was changing during the testing period. For example an eight hour 
combined temperature and tilt sweep test for a specific motion table position and wheel speed would first be subdivided into four temperature sections: hot; cooling; cold; heating. These temperature sections would then be further subdivided into tilt sections dependant on each of the five tilt conditions during each hour of testing and the extracted files would be labeled accordingly. This was done for ease of analysis of the data which will be discussed in the next Chapter.

The extracted files were then examined with MatLab ${ }^{T M}$. Each of the nine measured variables as discussed in Section 3.2.4 were examined graphically looking for "bad data points" or values that were either NaN (Not a Number) or clearly outside of the normal operating range $(\geq 5 \sigma)$ due to noise in the system. The entire data point for all nine variables was eliminated when a "bad data point" was found. Given the small number of data points eliminated and the high sampling rate of the GyroWheel ${ }^{T M}$ system the elimination of these data points had no effect on the results achieved in the calibration. 


\section{Chapter 6}

\section{Analysis and Conclusions}

This Chapter presents the analysis carried out on the data collected for GyroWheel TM calibration as well as the resulting temperature calibration coefficients and conclusions.

\subsection{Singular Value Decomposition}

The GyroWheel ${ }^{T M}$ torque coil current data from various tests was analyzed utilizing a least squares estimate solved using singular value decomposition, a method as outlined by Staley [24]. Simplified, the result of the calibration from multi-positional testing is a set of direction cosines that are written in the form of a transformational matrix, $C$. It is then necessary to take the pseudo-inverse of $C$ so that from the torque coil current measurements provided by the GyroWheel ${ }^{T M}$, the various ground calibration coefficients can be determined. A direct way to calculate this pseudo-inverse is to use the singular value decomposition of the matrix $C$, such that:

$$
\mathrm{C}=\mathrm{U} \Sigma \mathrm{V}^{\mathrm{T}}
$$


where $U$ and $V$ are orthogonal matrices and $\Sigma$ is a diagonal matrix. Then the pseudo-inverse, $C^{*}$, is easily found as

$$
C^{*}=V \Sigma^{-1} U^{T}
$$

where the elements of the inverse of the diagonal elements are just the reciprocals.

Specifically, the single value decomposition method utilized to solve the following overdetermined systems for a DTG is shown below. This analysis method requires an overdetermined system of equations. If the values calculated are put back into the equations shown below, one will not get the exact data back, but a very close approximation due to the least squares estimate. As discussed previously, the GyroWheel ${ }^{T M}$ behaves similarly to a DTG, therefore the following analysis will aid in GyroWheel ${ }^{\mathrm{TM}}$ calibration understanding. For the $\mathrm{X}$ rate input axis:

$$
\left\{\boldsymbol{i}_{y}\right\}=\left[\mathrm{C}_{\mathrm{x}}\right]\left\{\boldsymbol{X}_{x}\right\} / k_{y}
$$

and for the $\mathrm{Y}$ rate input axis:

$$
\left\{\boldsymbol{i}_{x}\right\}=\left[C_{\mathrm{y}}\right]\left\{\boldsymbol{X}_{y}\right\} / k_{x}
$$

where $\left\{\boldsymbol{i}_{y}\right\}$ is an $11 \times 1$ vector of the 11 position average $i_{y}$ currents and $\left\{\boldsymbol{i}_{x}\right\}$ is an $11 \times 1$ vector of the 11 position average $i_{x}$ currents; $k_{y}$ is the scale factor for the torquer coils physically located on the $\mathrm{X}$ axis and sensing the rate about the $\mathrm{X}$ axis and $k_{x}$ is the scale factor for the torquer coils physically located on the $\mathrm{Y}$ axis and sensing the rate about the $\mathrm{Y}$ axis. As defined, the currents $i_{y}$ and $i_{x}$ are in the coils physically located on the $\mathrm{X}$ and $\mathrm{Y}$ axis respectively.

$\left[C_{x}\right]$ is an $11 \times 10$ coefficient matrix with each row of $\left[C_{x}\right]$ consisting of the following 10 elements. Each column relates to one of the 11 multi-position tests:

$$
\left\{1, \mathrm{~g} \mathrm{C} \delta_{x D}, \mathrm{gC} \delta_{y D}, \mathrm{~g} \mathrm{C} \delta_{z D}, \mathrm{~g}^{2} \mathrm{C}^{2} \delta_{x D}, \mathrm{~g}^{2} \mathrm{C}^{2} \delta_{z D}, \mathrm{~g}^{2} \mathrm{C} \delta_{x D} \mathrm{C} \delta_{y D}, \omega_{x}, \omega_{y}, \omega_{z}\right\}
$$

and $\left[C_{y}\right]$ is an $11 \times 10$ coefficicnt matrix with each row of $\left[C_{y}\right]$ consisting of the following 10 elements. Each column relates to one of the 11 multi-position tests:

$$
\left\{1, \mathrm{~g} \mathrm{C} \delta_{y D}, \mathrm{gC} \delta_{x D}, \mathrm{gC} \delta_{z D}, \mathrm{~g}^{2} \mathrm{C}^{2} \delta_{y D}, \mathrm{~g}^{2} \mathrm{C}^{2} \delta_{z D}, \mathrm{~g}^{2} \mathrm{C} \delta_{x D} \mathrm{C} \delta_{y D}, \omega_{x}, \omega_{y}, \omega_{z}\right\}
$$


where $\mathrm{g}$ is the acceleration due to gravity and is taken as $9.81 \mathrm{~m} / \mathrm{s}^{2} ; \mathrm{C} \delta_{x D}=\cos \delta_{x D}$, and $\delta_{x D}$ is the angle made by the table system $\mathrm{X}$ axis and the down direction for the given position; $\mathrm{C} \delta_{y D}=\cos \delta_{y D}$, and $\delta_{y D}$ is the angle made by the table system $\mathrm{Y}$ axis and the down direction for the given position; $\mathrm{C} \delta_{z D}=\cos \delta_{z D}$, and $\delta_{z D}$ is the angle made by the table system $\mathrm{Z}$ axis and the down direction for the given position. Additionally:

$$
\begin{aligned}
\cos \delta_{x D} & =-\sin \psi \sin \phi \\
\cos \delta_{y D} & =\cos \psi \sin \phi \\
\cos \delta_{z D} & =\cos \phi
\end{aligned}
$$

where $\psi$ is the table azimuth angle (under a Left Hand Convention) and $\phi$ is the table elevation angle (under a Right Hand Convention).

From above, $\omega_{x}, \omega_{y}, \omega_{z}$ are the components of the Earth rate as seen by the table system axes for the given test position. This is a function of latitude and the table position (azimuth, $\psi$ and elevation, $\phi$ ). They are expressed as:

$$
\begin{aligned}
\omega_{x} & =\omega_{e}[-\sin \psi \cos \phi \cos \lambda+\sin \psi \sin \phi \sin \lambda] \\
\omega_{y} & =\omega_{e}[\cos \psi \cos \phi \cos \lambda-\cos \psi \sin \phi \sin \lambda] \\
\omega_{z} & =\omega_{e}[-\sin \phi \cos \lambda-\cos \phi \sin \lambda]
\end{aligned}
$$

where $\omega_{e}$ is the Earth's rotation rate of $\frac{2 \pi \mathrm{rad}}{86164 \mathrm{sec}}$ and $\lambda$ is the latitude of the motion table, $45.359^{\circ} \mathrm{N}$.

Finally, $\left\{\boldsymbol{X}_{x}\right\}$ from Equation 6.3 is an unknown $10 \times 1$ vector with the following components, most of which are the crror terms defined in Equation 5.1:

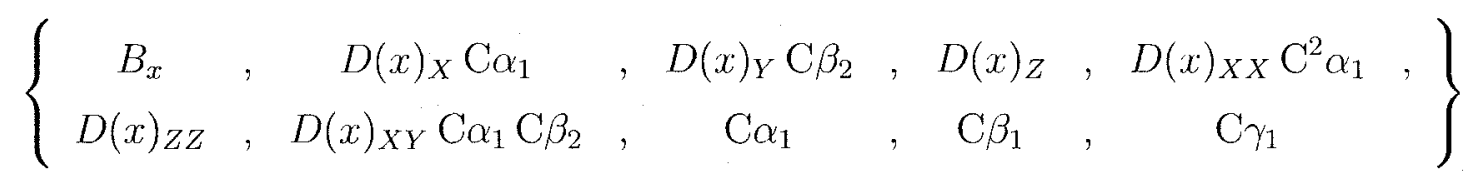

and $\left\{\boldsymbol{X}_{y}\right\}$ from Equation and 6.4 is an unknown $10 \times 1$ vector with the following 
components, most of which are the error terms defined in Equation 5.2:

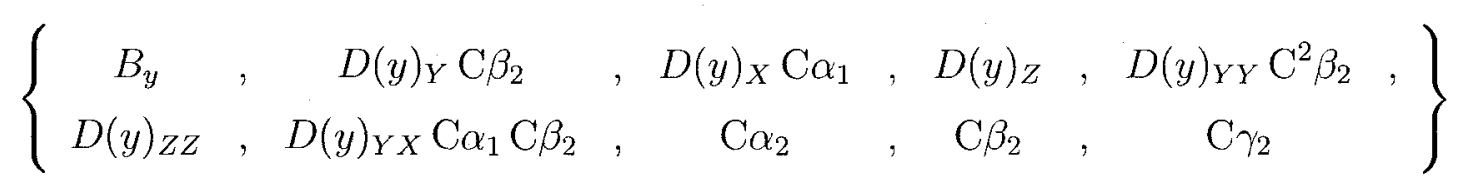

$\mathrm{C} \alpha_{1}=\cos \alpha_{1}$, where $\alpha_{1}$ is the misalignment angle between the $\mathrm{X}$ table system axis and the $\mathrm{X}$ GyroWheel ${ }^{\mathrm{TM}}$ rate input axis; $\mathrm{C} \beta_{1}=\cos \beta_{1}$, where $\beta_{1}$ is the misalignment angle between the $\mathrm{Y}$ table system axis and the $\mathrm{X}$ GyroWheel ${ }^{\mathrm{TM}}$ rate input axis; $\mathrm{C} \gamma_{1}=\cos \gamma_{1}$, where $\gamma_{1}$ is the misalignment angle between the $\mathrm{Z}$ table system axis and the $\mathrm{X}$ GyroWheel ${ }^{\mathrm{TM}}$ rate input axis; $\mathrm{C} \alpha_{2}=\cos \alpha_{2}$, where $\alpha_{2}$ is the misalignment angle between the $\mathrm{X}$ table system axis and the $\mathrm{Y}$ GyroWheel ${ }^{\mathrm{TM}}$ rate input axis; $\mathrm{C} \beta_{2}=\cos \beta_{2}$, where $\beta_{2}$ is the misalignment angle between the $\mathrm{Y}$ table system axis and the Y GyroWheel ${ }^{\mathrm{TM}}$ rate input axis; $\mathrm{C} \gamma_{2}=\cos \gamma_{2}$, where $\gamma_{2}$ is the misalignment angle between the $\mathrm{Z}$ table system axis and the $\mathrm{Y}$ GyroWheel ${ }^{\mathrm{TM}}$ rate input axis.

Equations 6.3 and 6.4 can be rewritten for the $\mathrm{X}$ rate input axis as:

$$
\left\{\boldsymbol{X}_{x}\right\}=\left[\mathrm{C}_{\mathbf{x}}\right]^{*} k_{y}\left\{\boldsymbol{i}_{y}\right\}
$$

and for the $\mathrm{Y}$ rate input axis as:

$$
\left\{\boldsymbol{X}_{y}\right\}=\left[C_{\mathrm{y}}\right]^{*} k_{x}\left\{\boldsymbol{i}_{x}\right\}
$$

Therefore given $\left\{\boldsymbol{i}_{y}\right\}$ and $\left\{\boldsymbol{i}_{x}\right\}$ these two overdetermined equations can be solved by finding the pseudo-inverse matrices $\left[C_{x}\right]^{*}$ and $\left[C_{y}\right]^{*}$ utilizing the MatLab ${ }^{T M}$ tools SVD.

The equations presented above are utilized to calibrate a DTG and are a simplification of Equations 5.1 and 5.2 that assumes null tilt conditions, $\phi_{x}$ and $\phi_{y}=0$, and therefore does not include the dynamic stiffness term $K_{\text {dyn }}$, the damping term $c_{g}$, or the transverse inertia term $I_{t}$. Also, the torquer misalignments are assumed to be negligible. The full equations for GyroWheel ${ }^{\text {TM }}$ consist of a system of 52 terms in $\left\{\boldsymbol{X}_{x}\right\}$ and $\left\{\boldsymbol{X}_{y}\right\}$ each plus the torquer misalignments $\alpha$ and $\beta$ as described in Section 4.4.2. This also does not yet include the temperature calibration. 


\subsection{Temperature Calibration}

For the temperature calibration the data was separated into distinct data packages, as discussed in Section 5.3, where the current in the torque coils, $i_{x}$ and $i_{y}$ was measured for various changes in temperature, $T_{h, y}$ and $T_{h, x}$, both when heating and cooling. The wheel speed, tilt conditions, and table position remain constant within the distinct data packages. The data was then graphed with $i_{x}$ versus $T_{h, y}$ and $i_{y}$ versus $T_{h, x}$. The current data plot when heating was averaged to plot a heating sweep line, and the current data plot when cooling was averaged to plot a cooling sweep line. Examples of these plots are shown in Figures 6.1 and 6.2 for $i_{x}$ versus $T_{h, y}$ and $i_{y}$ versus $T_{h, x}$ respectively, where $\omega_{z}=157 \mathrm{rad} / \mathrm{s}$; rotor tilt: $\phi_{x}=-1.0606^{\circ}$ and $\phi_{y}=1.0606^{\circ}$; and the table is position 4 of an 11 position test. The current in the torque coils is higher when conducting a heating cycle than when conducting a cooling cycle. This can be explained in relation to the Thermal Time Constant, $\tau$ as discussed in Section 2.1.2 in that the GyroWheel ${ }^{\mathrm{TM}}$ thermal time constant is relatively large at $65-72$ minutes.

Figures 6.1 and 6.2 also show an average plot of the heating sweep line with the cooling sweep line. The figures also show steady state testing conducted at specific temperatures. Steady state temperature tests meet the criteria that the temperature varied less than $0.05{ }^{\circ} \mathrm{C} / \mathrm{hr}$ during testing. The average plot of the heating and cooling sweep line is in close proximity to the plotted results for steady state temperature tests. Therefore from this and similar analysis it was determined to utilize the average plot of the heating and cooling sweep lines as the torque coil current value for a given housing temperature at each fixed wheol spced, tilt, condition, and table position.

A large portion of the data used in the analysis was taken from the combined temperature and tilt sweep testing as discussed in Section 5.2.7. The same procedure discussed above, utilizing the average of the heating and cooling sweep lines for 


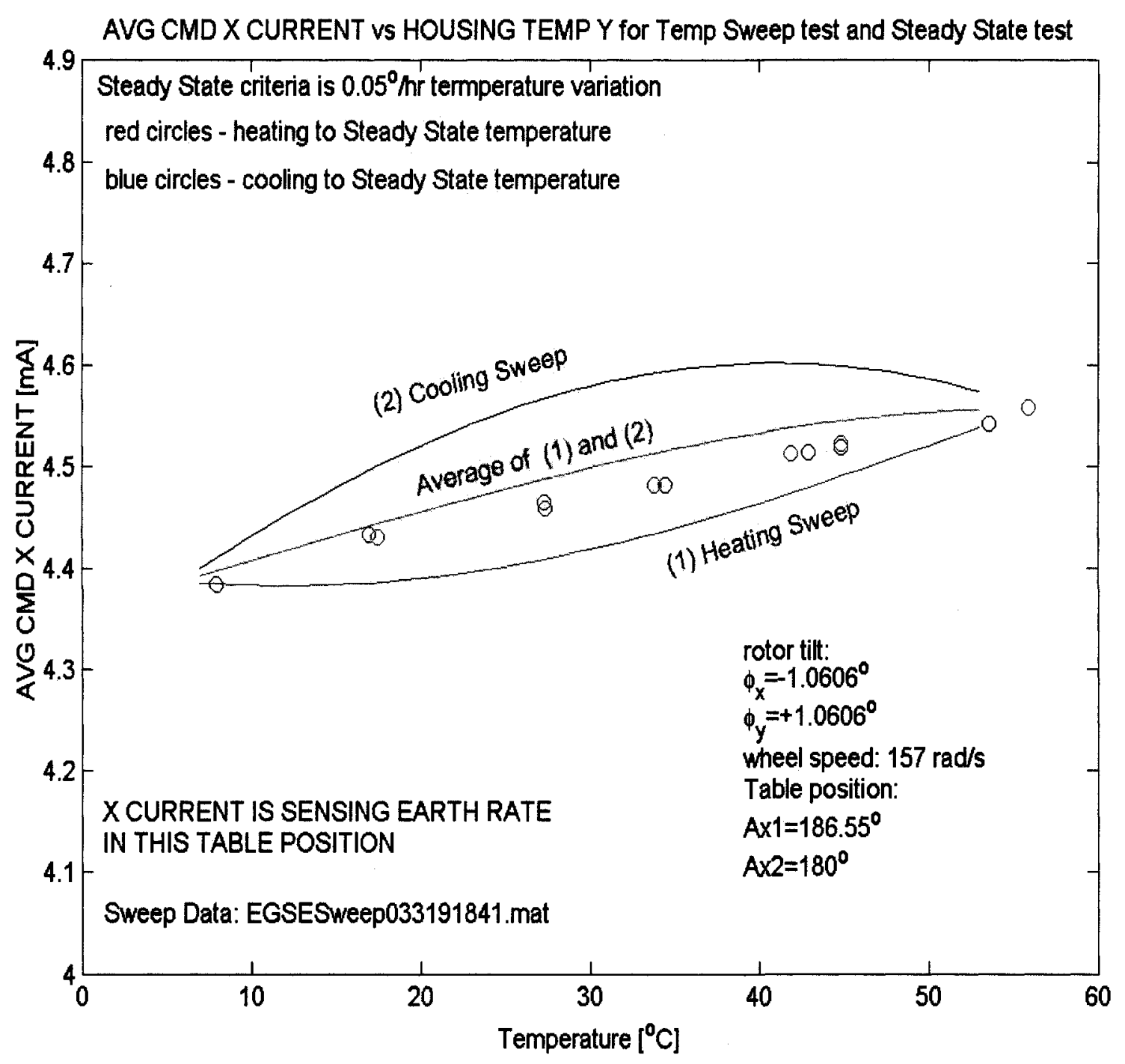

Figure 6.1: Average current in the torque coils physically located on the GyroWheel ${ }^{\mathrm{TM}} \mathrm{x}$ case axis Versus the Housing temperature $\mathrm{y}$ value in degrees Celsius for a Temperature Sweep Test and Steady State Tests.

Note: during this testing there was a $6.55^{\circ}$ offset in Axis 1 


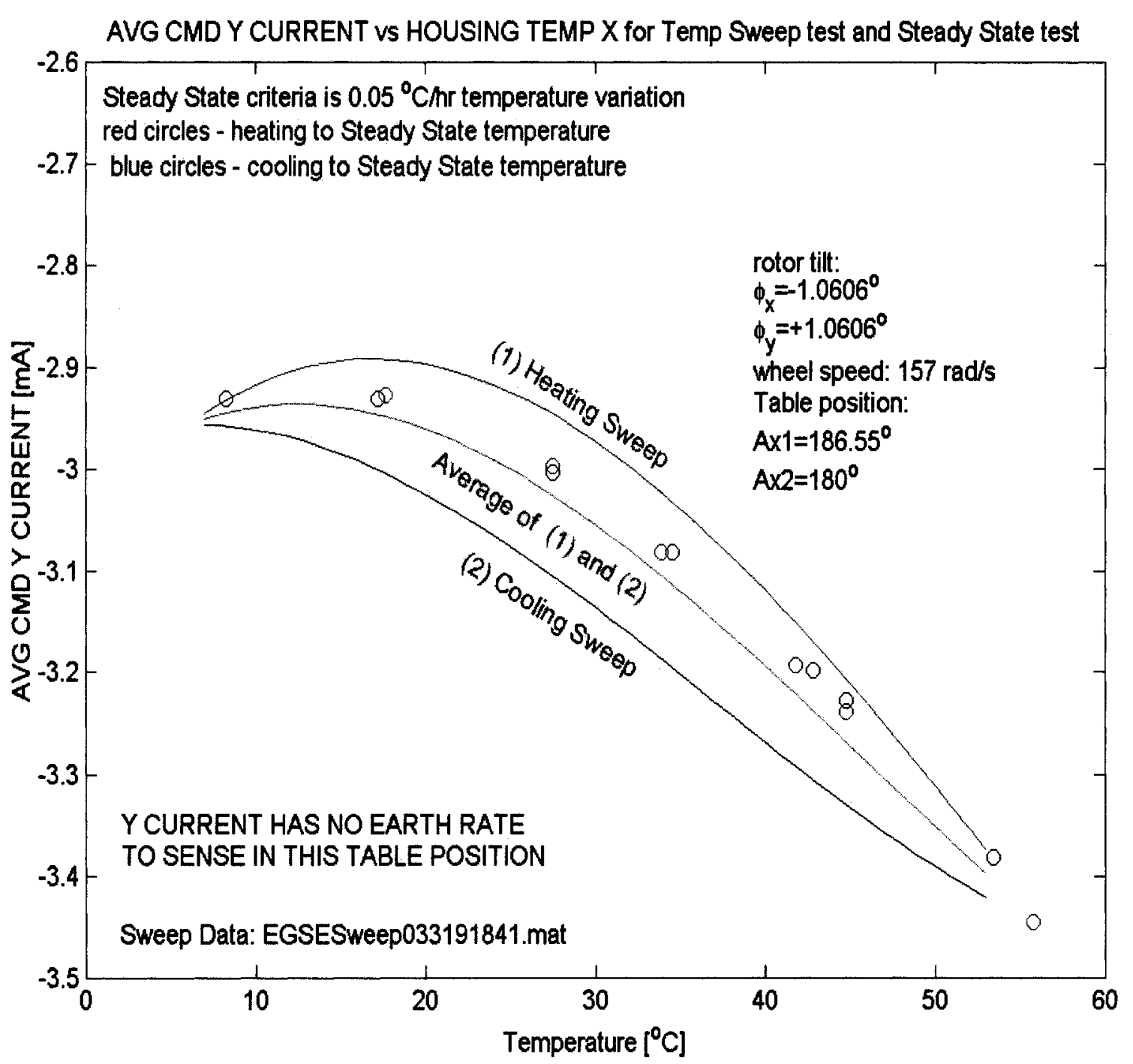

Figure 6.2: Average current in the torque coils physically located on the GyroWheel ${ }^{\mathrm{TM}} \mathrm{y}$ case axis Versus the Housing temperature $\mathrm{x}$ value in degrees Celsius for a Temperature Sweep Test and Steady State Tests.

Note: during this testing there was a $6.55^{\circ}$ offset in Axis 1 
determining the torque coil current value for a given housing temperature at each fixed wheel speed, tilt condition, and table position, was employed in the analysis of this data. The only difference was that the sweep lines were interpolated with a best curve to accommodate for the 44-49 minutes of missing data every hour of testing for each specific tilt position. A set of plots showing the original data scatter; the cooling sweep line; the heating sweep line; and their average for each of the first eight positions of an eleven position test, where $\omega_{z}=130 \mathrm{rad} / \mathrm{s}$; and rotor tilt: $\phi_{x}$ $=3^{\circ}$ and $\phi_{y}=0^{\circ}$ is shown in Appendix B.

The average currents as a function of temperature for each of the 11 positions were utilized as the values in $\left\{\boldsymbol{i}_{y}\right\}$ and $\left\{\boldsymbol{i}_{x}\right\}$ for a singular value decomposition analysis as described in Section 6.1. The output of this analysis for the GyroWheel ${ }^{T M}$ is $\left\{\boldsymbol{X}_{x}\right\}$ and $\left\{\boldsymbol{X}_{y}\right\}$ that contain all the cocfficients in Equations 5.1 and 5.2 as a function of temperature. These calibration coefficients as a function of temperature exist for each wheel speed and tilt position tested. Early results from this testing were analyzed to determine an initial polynomial order for all temperature calibrated terms for the in-flight rate sensing equations presented below as Equations 6.9 and 6.10. To determine the initial polynomial order for the temperature calibrated terms, the data was initially fit with a high order polynomial ( $7^{\text {th }}$ order). The polynomial order was then truncated, while reviewing changes to the calibration coefficients, at a point where the numerical impact of the higher order terms being truncated were not significant to the level of accuracy supported in the GyroWhecl TM. For paired coefficients, such as $B_{x}$ and $B_{y}$, the highest order required for one term was utilized for both. For example, changes the $5^{\text {th }}$ order term produced on $B_{x}$ were significant to the level of accuracy supported in the GyroWheel ${ }^{\mathrm{TM}}$, but were not significant for $B_{y}$. Only the $4^{\text {th }}$ order term for $B_{y}$ produced changes significant to the level of accuracy supported in the GyroWheel ${ }^{T M}$. In this instance both calibration cocfficicnts were chosen as $5^{\text {th }}$ order polynomials. 


\subsection{In-Flight Rate Sensing Temperature Calibrated Terms}

Equations 5.1 and 5.2 are the ground calibration equations for the GyroWheel ${ }^{\mathrm{TM}}$ system. The error calibration terms:

$$
\left(\begin{array}{ccccccccccc}
D(x)_{X} & , & D(y)_{Y} & , & D(x)_{Y} & , & D(y)_{X} & , & D(x)_{Z} & , & D(y)_{Z} \\
D(x)_{X X} & , & D(y)_{Y Y} & , & D(x)_{Z Z} & , & D(y)_{Z Z} & , & D(x)_{X Y} & , & D(y)_{Y X}
\end{array}\right)
$$

are functions of temperature with a minimum $3^{\text {rd }}$ order polynomial requirement to appropriately model their functions over the GyroWheel ${ }^{\mathrm{TM}}$ operating range. Inflight, the error terms related to $g$ are negligible and the GyroWheel ${ }^{T M}$ in flight rate sensing equations are:

$$
\begin{aligned}
& \omega_{c X}=\frac{1}{\omega_{z}}\left[k_{x} i_{x} \cos (\alpha+\beta)+k_{y} i_{y} \sin (\alpha)+c_{g, y} \dot{\phi}_{y}+K_{\mathrm{dyn}, y} \phi_{y}\right]-I_{t, x} \dot{\phi}_{x}-2 c_{g, x} \phi_{x}-B_{x} \\
& \omega_{c Y}=\frac{1}{\omega_{z}}\left[k_{y} i_{y} \cos (\alpha)-k_{x} i_{x} \sin (\alpha+\beta)-c_{g, x} \dot{\phi}_{x}-K_{\mathrm{dyn}, x} \phi_{x}\right]-I_{t, y} \dot{\phi}_{y}-2 c_{g, y} \phi_{y}-B_{y}
\end{aligned}
$$

The terms in Equations 6.9 and 6.10 can be separated into "calibrated" and "measured" values. The measured values are as discussed in Section 3.2.4. The calibrated values are dependent solely on temperature and will be denoted with the prime symbol ( $I$ ). The functional relationships with temperature as discussed above are either $2^{\text {nd }}, 3^{\text {rd }}$, or $5^{\text {th }}$ order polynomials and are defined below. Equations 6.9 and 6.10 therefore become:

$$
\begin{aligned}
& \omega_{c X}=\frac{1}{\omega_{z}}\left[k_{x}^{\prime} i_{x} \cos ^{\prime}(\alpha+\beta)+k_{y}^{\prime} i_{y} \sin ^{\prime}(\alpha)+c_{g, y}^{\prime} \dot{\phi}_{y}+K_{\mathrm{dyn}, y}^{\prime} \phi_{y}\right]-I_{t, x}^{\prime} \dot{\phi}_{x}-2 c_{g, x}^{\prime} \phi_{x}-B_{x}^{\prime} \\
& \omega_{c Y}=\frac{1}{\omega_{z}}\left[k_{y}^{\prime} i_{y} \cos ^{\prime}(\alpha)-k_{x}^{\prime} i_{x} \sin ^{\prime}(\alpha+\beta)-c_{g, x}^{\prime} \dot{\phi}_{x}-K_{\mathrm{dyn}, x}^{\prime} \phi_{x}\right]-I_{t, y}^{\prime} \dot{\phi}_{y}-2 c_{g, y}^{\prime} \phi_{y}-B_{y}^{\prime}
\end{aligned}
$$

where:

1. Bias

$B_{x}^{\prime}$ is a $5^{\text {th }}$ order polynomial of temperature, $T_{h, y}$, of the form:

$$
B_{x}^{\prime}=a_{0}+a_{1} T_{h, y}+a_{2} T_{h, y}^{2}+a_{3} T_{h, y}^{3}+a_{4} T_{h, y}^{4}+a_{5} T_{h, y}^{5}
$$


where $a_{i=0 \rightarrow 5} \in \mathrm{R}$.

$B_{y}^{\prime}$ is a $5^{\text {th }}$ order polynomial of temperature, $T_{h, x}$, of the form:

$$
B_{y}^{\prime}=b_{0}+b_{1} T_{h, x}+b_{2} T_{h, x}^{2}+b_{3} T_{h, x}^{3}+b_{4} T_{h, x}^{4}+b_{5} T_{h, x}^{5}
$$

where $b_{i=0 \rightarrow 5} \in \mathrm{R}$.

2. Torquer Misalignment Angles

There are four constants based on misalignments of the torquer axes with the rate sensing input axes. These terms are $3^{\text {rd }}$ order polynomial functions of temperature. To avoid the requirement for generating sine and cosine functions these terms are defined as follows:

$$
\begin{aligned}
\cos ^{\prime}(\alpha)=c_{0}+c_{1} T_{h, y}+c_{2} T_{h, y}^{2}+c_{3} T_{h, y}^{3}, \text { where } c_{i=0 \rightarrow 3} & \in \mathrm{R} ; \\
\cos ^{\prime}(\alpha+\beta)=d_{0}+d_{1} T_{h, x}+d_{2} T_{h, x}^{2}+d_{3} T_{h, x}^{3}, \text { where } d_{i=0 \rightarrow 3} & \in \mathrm{R} ; \\
\sin ^{\prime}(\alpha)=e_{0}+e_{1} T_{h, y}+e_{2} T_{h, y}^{2}+e_{3} T_{h, y}^{3}, \text { where } e_{i=0 \rightarrow 3} & \in \mathrm{R} ; \\
\sin ^{\prime}(\alpha+\beta)=f_{0}+f_{1} T_{h, x}+f_{2} T_{h, x}^{2}+f_{3} T_{h, x}^{3}, \text { where } f_{i=0 \rightarrow 3} & \in \mathrm{R} ;
\end{aligned}
$$

3. Scale Factors

$k_{x}^{\prime}$ is a $5^{\text {th }}$ order polynomial of temperature, $T_{h, x}$, of the form:

$$
k_{x}^{\prime}=g_{0}+g_{1} T_{h, x}+g_{2} T_{h, x}^{2}+g_{3} T_{h, x}^{3}+g_{4} T_{h, x}^{4}+g_{5} T_{h, x}^{5}
$$

where $g_{i=0 \rightarrow 5} \in \mathrm{R}$.

$k_{y}^{\prime}$ is a $5^{\text {th }}$ order polynomial of temperature, $T_{h, y}$, of the form:

$$
k_{y}^{\prime}=h_{0}+h_{1} T_{h, y}+h_{2} T_{h, y}^{2}+h_{3} T_{h, y}^{3}+h_{4} T_{h, y}^{4}+h_{5} T_{h, y}^{5}
$$

where $h_{i=0 \rightarrow 5} \in \mathrm{R}$.

4. Transverse Inertias

$I_{t, x}^{\prime}$ is a $2^{\text {nd }}$ order polynomial of temperature, $T_{h, x}$, of the form:

$$
I_{t, x}^{\prime}=l_{0}+l_{1} T_{h, x}+l_{2} T_{h, x}^{2}, \text { where } l_{i=0 \rightarrow 2} \in \mathrm{R}
$$


$I_{t, y}^{\prime}$ is a $2^{\text {nd }}$ order polynomial of temperature, $T_{h, y}$, of the form:

$$
I_{t, y}^{\prime}=m_{0}+m_{1} T_{h, y}+m_{2} T_{h, y}^{2}, \text { where } m_{i=0 \rightarrow 2} \in \mathrm{R}
$$

\section{Damping Terms}

$c_{g, x}^{\prime}$ is a $3^{\text {rd }}$ order polynomial of temperature, $T_{h, x}$, of the form:

$$
c_{g, x}^{\prime}=n_{0}+n_{1} T_{h, x}+n_{2} T_{h, x}^{2}+n_{3} T_{h, x}^{3}, \text { where } n_{i=0 \rightarrow 3} \in \mathrm{R}
$$

$c_{g, y}^{\prime}$ is a $3^{\text {rd }}$ order polynomial of temperature, $T_{h, y}$, of the form:

$$
c_{g, y}^{\prime}=p_{0}+p_{1} T_{h, y}+p_{2} T_{h, y}^{2}+p_{3} T_{h, y}^{3}, \text { where } p_{i=0 \rightarrow 2} \in \mathrm{R}
$$

6. Dynamic Stiffness Terms

$K_{\mathrm{dyn}, x}^{\prime}$ is a $3^{\text {rd }}$ order polynomial of temperature, $T_{h, x}$, of the form:

$$
K_{\mathrm{dyn}, x}^{\prime}=u_{0}+u_{1} T_{h, x}+u_{2} T_{h, x}^{2}+u_{3} T_{h, x}^{3}, \text { where } u_{i=0 \rightarrow 3} \in \mathrm{R}
$$

$K_{\mathrm{dyn}, y}^{\prime}$ is a $3^{\text {rd }}$ order polynomial of temperature, $T_{h, y}$, of the form:

$$
K_{\mathrm{dyn}, y}^{\prime}=v_{0}+v_{1} T_{h, y}+v_{2} T_{h, y}^{2}+v_{3} T_{h, y}^{3}, \text { where } v_{i=0 \rightarrow 2} \in \mathrm{R}
$$

The 62 various coefficients needed to implement this temperature calibrated rate sensing algorithm are summarized in Table 6.1. The implementation of this algorithm above was based on only a small sampling of data and many polynomial coefficients may be zero pending further analysis of rate sensing data. Appendix $\mathrm{C}$ shows graphs of the calibration coefficients $B_{x}^{\prime}, B_{y}^{\prime}, k_{x}^{\prime}, k_{y}^{\prime}, c_{g, x}^{\prime}, c_{g, y}^{\prime}, K_{\mathrm{dyn}, x}^{\prime}$, and $K_{\mathrm{dyn}, y}^{\prime}$ versus temperature for each of the three wheel speed at the null tilt condition: $\phi_{x}=$ $0^{\circ}$ and $\phi_{y}=0^{\circ}$.

\subsection{Final Calibration Coefficients}

For the final analysis, the data for each of the in-flight temperature calibration coefficients from each wheel speed and tilt condition, was utilized to determine the 
Table 6.1: The Various Coefficients Needed to Implement the GyroWheel ${ }^{\text {TM }}$ In-Flight Temperature Calibrated Rate Sensing Algorithm

\begin{tabular}{|c|c|}
\hline Variable & Polynomial Coefficients \\
\hline $\mathrm{X}$ axis bias, $B_{x}^{\prime}$ & 6 \\
\hline $\mathrm{Y}$ axis bias, $B_{y}^{\prime}$ & 6 \\
\hline $\mathrm{X}$ axis misalignment $\operatorname{cosine}, \cos ^{\prime}(\alpha)$ & 4 \\
\hline $\mathrm{Y}$ axis misalignment cosine, $\cos ^{\prime}(\alpha+\beta)$ & 4 \\
\hline $\mathrm{X}$ axis misalignment sine, $\sin ^{\prime}(\alpha)$ & 4 \\
\hline $\mathrm{Y}$ axis misalignment sine, $\sin ^{\prime}(\alpha+\beta)$ & 4 \\
\hline $\mathrm{X}$ axis scale factor, $k_{x}^{\prime}$ & 6 \\
\hline $\mathrm{Y}$ axis scale factor, $k_{y}^{\prime}$ & 6 \\
\hline $\mathrm{X}$ axis transverse inertia, $I_{t, x}^{\prime}$ & 3 \\
\hline $\mathrm{Y}$ axis transverse inertia, $I_{t, y}^{\prime}$ & 3 \\
\hline $\mathrm{X}$ axis damping, $c_{g, x}^{\prime}$ & 4 \\
\hline $\mathrm{Y}$ axis damping, $c_{g, y}^{\prime}$ & 4 \\
\hline $\mathrm{X}$ axis dynamic stiffness, $K_{\mathrm{dyn}, x}^{\prime}$ & 4 \\
\hline $\mathrm{Y}$ axis dynamic stiffness, $K_{\mathrm{dyn}, y}^{\prime}$ & 4 \\
\hline
\end{tabular}


overall best fit of the data over the GyroWheel ${ }^{\mathrm{TM}}$ temperature, wheel speed, and tilt condition operating range. The final results are presented below:

1. Bias

$$
\begin{aligned}
B_{x}^{\prime} & =-6.571 \times 10^{-5} \\
& +6.757 \times 10^{-6} T_{h, y} \\
& -6.093 \times 10^{-7} T_{h, y}^{2} \\
& +2.022 \times 10^{-8} T_{h, y}^{3} \\
& -3.028 \times 10^{-10} T_{h, y}^{4} \\
& +1.751 \times 10^{-12} T_{h, y}^{5} \\
& \\
B_{y}^{\prime} & =-1.398 \times 10^{-5} \\
& -9.215 \times 10^{-7} T_{h, x} \\
& +9.324 \times 10^{-8} T_{h, x}^{2} \\
& -4.214 \times 10^{-9} T_{h, x}^{3} \\
& +7.991 \times 10^{-11} T_{h, x}^{4} \\
& -5.824 \times 10^{-13} T_{h, x}^{5}
\end{aligned}
$$

2. Torquer Misalignment Angles

$$
\begin{aligned}
\cos ^{\prime}(\alpha) & =1+0 T_{h, y}+0 T_{h, y}^{2}+0 T_{h, y}^{3} \\
\cos ^{\prime}(\alpha+\beta) & =1+0 T_{h, x}+0 T_{h, x}^{2}+0 T_{h, x}^{3} \\
\sin ^{\prime}(\alpha) & =0+0 T_{h, y}+0 T_{h, y}^{2}+0 T_{h, y}^{3} \\
\sin ^{\prime}(\alpha+\beta) & =0+0 T_{h, x}+0 T_{h, x}^{2}+0 T_{h, x}^{3}
\end{aligned}
$$


3. Scale Factors

$$
\begin{aligned}
k_{x}^{\prime} & =-18.12 \\
& +0.7595 T_{h, x} \\
& -0.05073 T_{h, x}^{2} \\
& +0.001500 T_{h, x}^{3} \\
& -\quad 2.107 \times 10^{-5} T_{h, x}^{4} \\
& +1.164 \times 10^{-7} T_{h, x}^{5}
\end{aligned}
$$

$$
\begin{aligned}
k_{y}^{\prime} & =13.28 \\
& +0.1134 T_{h, y} \\
& -0.007573 T_{h, y}^{2} \\
& +1.746 \times 10^{-4} T_{h, y}^{3} \\
& -1.121 \times 10^{-6} T_{h, y}^{4} \\
& -2.198 \times 10^{-9} T_{h, y}^{5}
\end{aligned}
$$

4. Transverse Inertias

$$
\begin{aligned}
& I_{t, x}^{\prime}=0.01030+0 T_{h, x}+0 T_{h, x}^{2} \\
& I_{t, y}^{\prime}=0.01030+0 T_{h, y}+0 T_{h, y}^{2}
\end{aligned}
$$

5. Damping Terms

$$
\begin{aligned}
c_{g, x}^{\prime} & =4.141 \times 10^{-4} \\
& +1.323 \times 10^{-6} T_{h, x} \\
& -4.337 \times 10^{-8} T_{h, x}^{2} \\
& +4.103 \times 10^{-10} T_{h, x}^{3}
\end{aligned}
$$




$$
\begin{aligned}
c_{g, y}^{\prime} & =3.727 \times 10^{-4} \\
& +4.379 \times 10^{-6} T_{h, y} \\
& -4.016 \times 10^{-8} T_{h, y}^{2} \\
& +8.375 \times 10^{-10} T_{h, y}^{3}
\end{aligned}
$$

6. Dynamic Stiffness Terms

$$
\begin{aligned}
K_{\text {dyn }, x}^{\prime} & =-1.766 \\
& -0.008190 T_{h, x} \\
& +2.130 \times 10^{-4} T_{h, x}^{2} \\
& -3.670 \times 10^{-6} T_{h, x}^{3} \\
K_{\text {dyn }, y}^{\prime} & =-1.811 \\
& +0.004532 T_{h, y} \\
& -5.610 \times 10^{-4} T_{h, y}^{2} \\
& +6.753 \times 10^{-6} T_{h, y}^{3}
\end{aligned}
$$

\subsubsection{Discussion of Final Results}

As expected, due to the changes in the strength of the magnetic field with temperature, as well as different thermal properties of the GyroWheel ${ }^{T M}$ flexures, the bias terms, $B$; scale factors, $k$; damping terms, $c_{g}$; and dynamic stiffness terms, $K_{\mathrm{dyn}}$ all vary with temperature.

The torquer misalignment angles, as shown above, do not vary with temperature, which is a result that makes sense due to the fact that changes in temperature should not have an effect on the torquer input axis direction. The results presented above do not mean that the torquer misalignment is equal to zero for the GyroWheel ${ }^{T M}$ system however. The best values for $\alpha$ and $\beta$ determined experimentally from analysis of four position multi-position test data are: $\alpha \approx 6.95^{\circ}$ and $\alpha+\beta \approx-6.42^{\circ}$. 
The results shown above in Equations 6.29 through 6.32 are simply a result of the algorithm utilized in the determination of temperature calibration that includes the previously experimentally determined misalignment angles.

Additionally the transverse inertias show no variation with temperature change and are both the same. The fact that the transverse inertia does not change with temperature makes sense given that the mass, shape, and alignment of the GyroWheel $^{\mathrm{TM}}$ should not change with temperature variation. The fact that both transverse inertias are the same is also most likely due to the fact that as stated in Equations 4.58 and 4.59

$$
I_{t}=\frac{\left(I_{r s}+I_{g t}\right)}{\left(I_{r s}+\frac{I_{g s}}{2}\right)}
$$

and in Section 4.3.4, the gimbal transverse inertias for both the $\mathrm{X}$ and $\mathrm{Y}$ axes were assumed to be equal and set to the value $I_{g t}$. This means that if there are no changes due to temperature that present themselves in the transverse inertias, then the values for both the $\mathrm{X}$ and $\mathrm{Y}$ axis will be equal, as above.

This research is valuable as not every DTG can be temperature controlled and similar methodology as applied in this thesis could be utilized to calibrate a DTG for temperature effects. As woll this project provides calibration methodology for DTGs operating outside of tuned conditions with respect to both wheel speed and tilt conditions.

\subsection{Summary of Contributions}

Contributions have been made in the areas of understanding and calibration of dynamically tuned gyros for temperature variation as well as operation outside of the tuning condition, which is known to be a function of both spin rate and tilt. Spccifically a process has been followed in the conduct of the calibration of the GyroWheel ${ }^{\mathrm{TM}}$ that can be applied when calibrating any dynamically tuned gyro, or complex momentum management system similar to the GyroWheel ${ }^{\mathrm{TM}}$, for both 
temperature effects and de-tuned operation.

The general process to be followed is presented below:

- Determine the operating parameters for the DTG including: whether it will be in a temperature controlled environment; the operational temperature range; if it will be operated outside of de-tuned conditions; and the operational tilt and speed ranges.

- Determine the measured values provided by the DTG. The measured values provided by GyroWheel ${ }^{\mathrm{TM}}$ are presented in Section 3.2.4.

- Examine the dynamics and mathematical models specific to the DTG including validating any simplifying assumptions based on the operating parameters. The dynamics of the GyroWheel ${ }^{\mathrm{TM}}$ system are presented in Section 4.3.

- Determine the in-flight rate sensing equations specific to the DTG. The GyroWheel TM in-flight rate sensing equations are shown as Equations 4.60 and 4.61.

- Add any required in-flight error terms to the in-flight rate sensing equations for the DTG. Ensure the absolute bias and torquer misalignment terms are accounted for. The GyroWheel ${ }^{\mathrm{TM}}$ in-flight rate sensing equations including in-flight error terms are shown as Equations 4.65 and 4.66.

- Since the DTG is most likely not going to be calibrated in space (in-flight) it will be calibrated on the ground. The presence of gravity on the ground acts as an acceleration, aggravating various error terms that are normally negligible in micro-gravity, therefore error terms as discussed in Section 4.1.3, and shown in Equations 4.29 and 4.30, need to also be added to the in-flight rate sensing equations for ground calibration. The GyroWheel ${ }^{\mathrm{TM}}$ ground calibration rate sensing equations including both the in-flight error terms and all additional ground (gravity) related error terms are shown as Equations 4.67 and 4.68. 
- Conduct multi-position testing to determine which, if any, of the ground related error terms can be deemed negligible as discussed in Section 5.2.3. This may then reduce the number of error terms to be calibrated which could also reduce the number of positions required when conducting multi-position testing as discussed in Section 4.2.1. The results from the multi-position testing should be analyzed utilizing the singular value decomposition method explained in Section 6.1 as if smaller and smaller subsets of the original multi-position tests were conducted. These results should then be compared. If the standard deviations of the resulting ground calibration error terms from the smaller subset of multi-position tests are decreasing or staying the same then the various ground calibration error terms additional to the previous subset should be deemed negligible. This was the case when comparing 15 position testing to 13 position testing for the GyroWheel ${ }^{T M}$. Conversely if the standard deviations calculated from the smaller subset of multi-position tests are increasing then the ground calibration error terms additional to the previous subset cannot be deemed negligible. This was the case when comparing 11 position testing to 8 position testing for the GyroWheel ${ }^{T M}$. Further explanation of this process is included in Appendix A

- Remove any ground calibration error terms deemed negligible from the ground calibration rate sensing equations. The GyroWheel ${ }^{\mathrm{TM}}$ final ground calibration rate sensing equations are shown as Equations 5.1 and 5.2.

- Gather the testing equipment and construct the testing apparatus. A motion table as well as a vacuum chamber are required. If calibrating for temperature, a heating and cooling system are also required. The equipment utilized for GyroWheel $^{\text {TM }}$ testing is described in Section 5.1.

- Develop the testing methodology, ensuring the maximum testing value is achieved in the minimum time. For example, as discussed in Section 5.2.7 the 
tilt sweep testing and temperature sweep testing for the GyroWheel ${ }^{\mathrm{TM}}$ were combined in order to shorten the time required for testing. The GyroWheel ${ }^{\mathrm{TM}}$ testing methodology is discussed in Section 5.2 .

- To aid in analysis, separate the data into distinct packages depending on which variable was changing during the testing period. The GyroWheel ${ }^{\mathrm{TM}}$ data is discussed in Section 5.3.

- Analyze the DTG data, dealing with the temperature calibration first. For a distinct data package, (constant wheel speed, tilt conditions, and table position) take an average of the current in the torque coils when heating to plot a heating sweep line and do the same when cooling to plot a cooling sweep line. Compare the heating sweep line and the cooling sweep line with steady state temperature testing. Should the average plot of the heating and cooling sweep lines correspond well with the steady state temperature testing, as in the case of the GyroWheel ${ }^{\mathrm{TM}}$ as described in Section 6.2, then it is acceptable to use an average of the heating and cooling sweep lines as the torque coil current value for a given temperature value. If not, determine a better correlation for the torque coil current in relation to temperature.

- Conduct a singular value decomposition, as discussed in Section 6.1, utilizing data from all required positions for multi-position testing. The current value as a function of temperature will be the average of the heating and cooling sweep line as discussed above for each position. The output of this analysis will be all the coefficients from the final ground calibration rate sensing equations as a function of temperature for each wheel speed and tilt position tested. As discussed in Section 6.2, further analyze each calibration coefficient with respect to temperature to determine the lowest polynomial order required to cnsure the accuracy of the calibration coefficients match the level of accuracy supported in the DTG. 
- Re-write the in-flight rate scnsing equations to denote the calibrated values dependent solely on temperature with the prime symbol ('). The GyroWheel ${ }^{T M}$ final in-flight rate sensing equations calibrating for temperature are shown as Equations 6.11 and 6.12 .

- Examine the data for each calibration coefficient from each wheel speed and tilt condition and determine the overall best fit of the data over the DTG temperature, wheel specd, and tilt condition operating range. The final inflight calibration coefficients for GyroWheel ${ }^{\mathrm{TM}}$ are shown in Section 6.4.

This process, if properly followed and tailored, will provide temperature calibrated and/or de-tuned in-flight rate sensing for any DTG.

\subsubsection{Future Work}

The next step required for the body of work on the GyroWheel ${ }^{\text {TM }}$ system would be to complete on-orbit calibration of the instrument. On-orbit calibration would provide a validation on all previous work conducted as well as highlight further areas for improvement in both the system and its calibration. 


\section{References}

[1] Ashworth, J.R., "Experimental Rescarches on Drawn Steel - Part I. The Influences of Changes of Temperature on Magnetism", Philosophical Transactions of the Royal Society of London. Series A, Containing Papers of a Mathematical or Physical Character, Vol. 201, 1903, pp. 1-35.

[2] Bender, J.D., Brehove, J.D. Momentum Wheel Platform Steering system and Method, United States Patent, No. 5,261,631, November 16, 1993.

[3] Benton, William. Gyroscope, article, Encyclopedia Britannica, Toronto, 1965, pp 47-51.

[4] Broxmeyer, Charles. Inertial Navigation Systems, McGraw-Hill Company, Toronto, 1964.

[5] Cain, J.S., "Investigation of the Crossed Flexure Pivot and the Dynamics of the Momentum Management System Spacecraft Control Component and the Dynamically Tuned Gyroscope", PhD Thesis, Department of Mechanical and Aerospace Engineering, Carleton University, Ottawa, February, 1999.

[6] Craig, R.J.G., "Theory of Operation of an Elastically Supported Tuned Gyroscope", IEEE Transactions on Aerospace and Electronic Systems, Vol. AES-8, No. 3, 1972, pp. 280-288.

[7] Craig, R.J.G., "Theory of Errors of a Multigimbal, Elastically Supported, Tuned Gyroscope", IEEE Transactions on Aerospace and Electronic Systems, Vol. AES-8, No. 3, 1972, pp. 289-297.

[8] Craig, R.J.G., "Dynamically Tuned Gyros in Strapdown Systems", AGARD Conference on inertial Navigation Computers and Systems, Florence, Italy. October, 1972, pp. 12-1 to 12-7.

[9] de Carufel, J., Spring, K., McCabe, D., "GyroWheel TM Rate Sensing Design", GyroWheel $^{T M}$ Technical Note Magellan Aerospace Corporation, Ottawa, January, 2000. 
[10] Dougherty, H., Rodden, J., Reschke, L.F., Tompetrini, K., Weinstein, S.P. and Slater, D. Performance Characterization of the Hubble Space Telescope Rate Gyro Assembly, IFAC Automatic Control in Space Symposium. Toulouse, France, 1985, pp. 219-222.

[11] Goldstein, H. Classical Dynamics, Addison Wesley Publishing Company, Reading, Massachusetts, 1980.

[12] Hartl, D.A., "Alignment Determination and Error Characterisation of Inertial Components for use in an Airborne Gravimeter", M.Eng. Thesis, Department of Mechanical and Aerospace Engineering, Carleton University, Ottawa, April, 1995.

[13] Howe, E.W., Savet, P.H., "The Dynamically Tuned Free Rotor Gyro", Control Engineering, June, 1964, pp. 67-72.

[14] "IEEE Specification Format Guide and Test Procedure for Two-Degrec-ofFreedom Dynamically Tuned Gyros", Prepared by the Gyro and Accelerometer Panel of the IEEE AESS., IEEE, New York, NY, 1989, pp. 59-65.

[15] Lawrence, A.W. Modern Inertial Technology, Springer-Verlag, New York, NY, 1993.

[16] Lawrence, A.W., "A Simulation of a Dynamically Tuned Gyroscope", Gyrodynamics: Euromech 38 Colloquim, University of Louvain, 1974, pp. 56-62.

[17] Ower, J.C., "Analysis and Control System Design of an Innovative Tuned-Rotor Instrument", PhD Thesis, Department of Mechanical and Aerospace Engineering, Carleton University, Ottawa, May, 2000.

[18] Parker, R.J., Advances in Permanent Magnetism, John Wiley and Sons, Inc., New York, 1990.

[19] Schultz, F., de Carufel, J., Staley, D., Vinnins, M. Rate Sensing Modelling and Calibration of the GyroWheel ${ }^{T M}$, ASTRO 2002 - 13th CASI Conference on Astronautics, November 2002, Ottawa, Ontario, Canada.

[20] Schultz, F., "GyroWheel ${ }^{\mathrm{TM}}$ Thermal Time Constant", Personal communication from Fred Schultz, 2002.

[21] Senez, M., Pokrupa, N., Taylor, B., Staley, D., Vinnins, M., The Gyro Wheel ${ }^{T M}$ Testing And Flight Qualification Program, AAS 02-047 - 25th Annual AAS Guidance and Control Conference, February 6-10 2002, Breckenridge, Colorado, U.S.A. 
[22] Staley, D.A. Orbital Mechanics and Spacecraft Control, Carleton University, Ottawa, 2002.

[23] Staley, D., Cain, J.S., "Dynamics of a Tuned Rotor Gyroscope", Canadian Aeronautics and Space Journal, Vol. 43, No. 2, 1997, pp. 106-110.

[24] Staley, D., "Ancon-R.860 HRU Alignment Calibration", Ancon Space Technology Corporation, Mississauga, 1993.

[25] Titterton, D.H. and Weston, J.L. Strapdown Inertial Navigation Technology, Institution of Electrical Engineers, 2nd edition, 2004.

[26] Tyc, G., Spring, K., Taylor, B., Staley, D., Vinnins, M. The GyroWheel TM Development and Flight Qualification Plan, ASTRO 2000 - 11th CASI Conference on Astronautics, November 2000, Ottawa, Ontario, Canada.

[27] Tyc, G., Staley, D., Whitehead, W., Pradhan, S., Ower, C., Cain, J., Wiktowy, M. GyroWheel ${ }^{T M}$ - An Innovative New Actuator/Sensor for 3-axis Spacecraft Attitude Control, SSC99-XI-8 - 13th Annual AIAA/USU Conference on Small Satellites, 1999, Ottawa, Ontario, Canada.

[28] Wertz, J.R., Larson, W.J. Space Mission Analysis and Design, Microcosm Press and Kluwer Academic Publishers, 3rd Edition, 1999.

[29] Wertz, James R. Spacecraft Attitude Determination and Control, Volume 73. D. Reidel Publishing Company, Boston, 1978.

[30] Wiktowy, M.J., "Development of a Tilt Sensor for the Momentum Management System", M.Eng. Thesis, Department of Mechanical and Aerospace Engineering, Carleton University, Ottawa, August, 1998.

[31] Wilkinson, R.H., "Testing of Two-Degree-of-Freedom Gyros", Appendix A to Chapter Precision Inertial Gyro Testing at MIT of Inertial Component Testing: Philosophy and Methods, AGARDograph No. 128. 1970. 


\section{Appendix A}

\section{Calibration Coefficients for}

\section{Various Multi-Position Analyses}

used to Determine Negligible

\section{Coefficient Terms}

The data presented in this Appendix was collected by Fred Schultz and comes from his logbook.

The following data presented in Table A.1 was collected on 7 September 2002 for the $i_{x}$ and $i_{y}$ values for GyroWheel ${ }^{\mathrm{TM}}$ tuned conditions $\left(\phi_{x}=0, \phi_{y}=0\right.$, and $\omega_{z}=157$ $\mathrm{rad} / \mathrm{s})$. The data is displayed graphically in Figure A.1. Figure A.1 also shows examples of some of the "bad data points" that were eliminated as discussed in Scction 5.3. The 16th position is a repeat of the first, position to ensure repeatability of the results.

The calibration coefficients calculated from the GyroWheel ${ }^{\mathrm{TM}}$ Command Data for the 15 position test described above are shown in Table A.2. The misalignment angles, $\alpha$ and $\beta$ are not the same as described in Section 4.4.2, where they are defined 


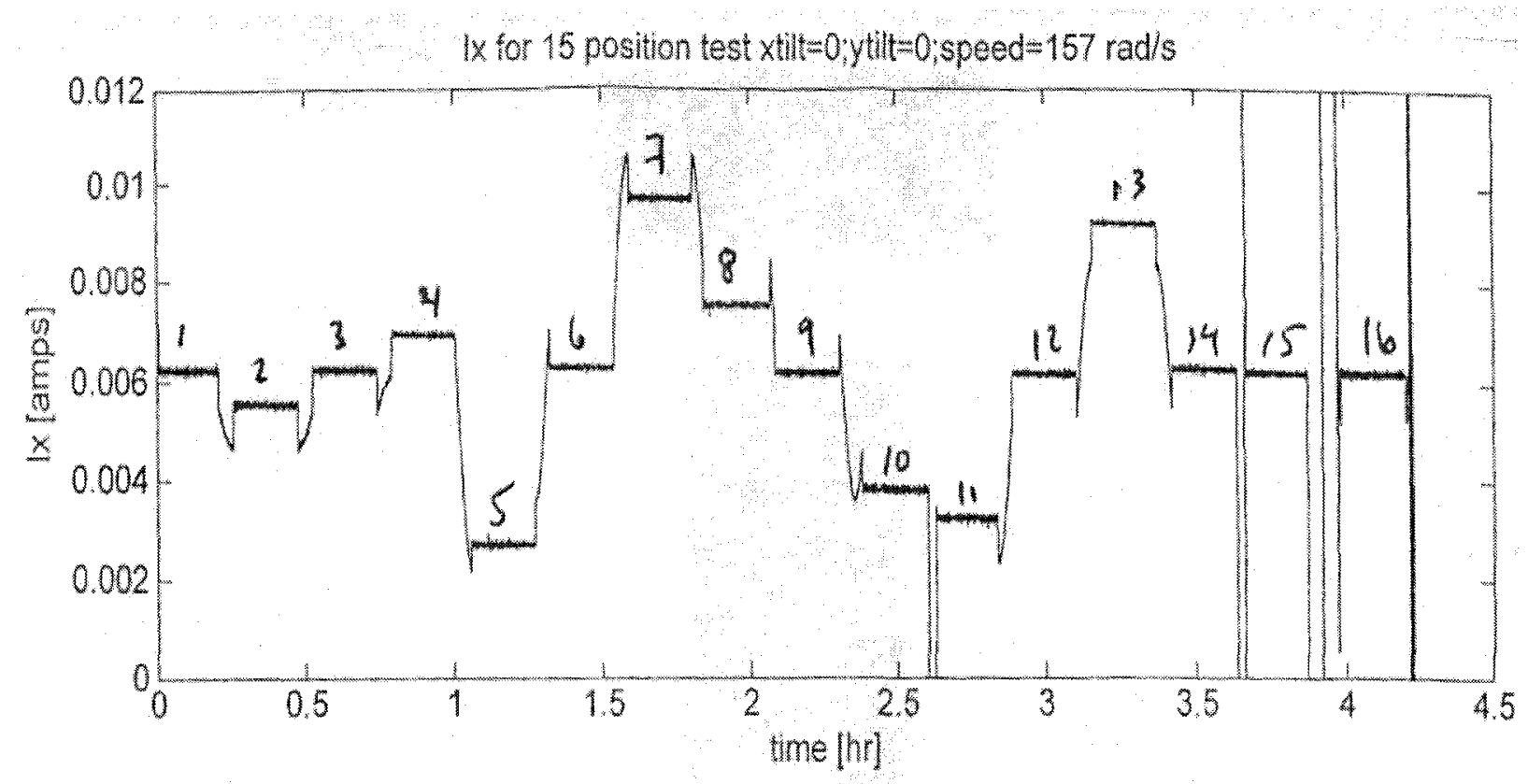

Iy for 15 position test xtill $=0$; ytitt $=0$; speed $=157 \mathrm{rad} / \mathrm{s}$

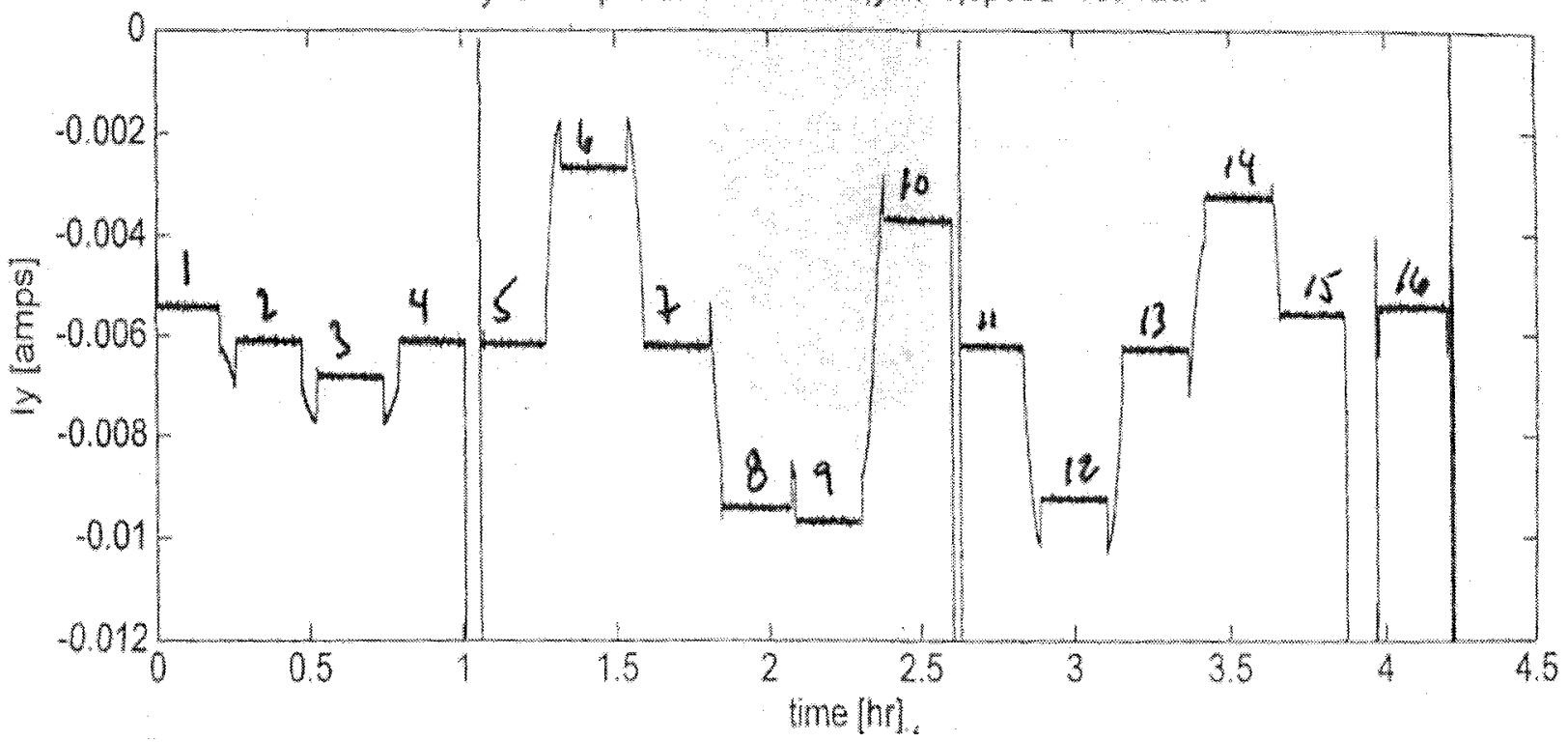

Figure A.1: GyroWheel ${ }^{\mathrm{TM}} i_{x}$ and $i_{y}$ values for a 15 position multi-position test conducted on the $7^{\text {th }}$ of September 2002 
APPENDIX A. CALIBRATION COEFFICIENTS FOR VARIOUS MULTI-POSITION ANALYSES USED TO DETERMINE NEGLIGIBLE COEFFICIENT TERMS

Table A.1: 15 position test (7 September 2002): $i_{x}$ and $i_{y}$ data

\begin{tabular}{|l|l|l|l|l|}
\hline \multicolumn{5}{|c|}{ GyroWheel TM Command Data } \\
\hline Position \# & $i_{x}[\mathrm{~mA}]$ & $\sigma i_{x}[\mathrm{~mA}]$ & $i_{y}[\mathrm{~mA}]$ & $\sigma i_{y}[\mathrm{~mA}]$ \\
\hline \hline 1 & 6.23628 & 0.03981 & -5.40994 & 0.03487 \\
\hline 2 & 5.53279 & 0.03672 & -6.11082 & 0.03675 \\
\hline 3 & 6.23061 & 0.03770 & -6.81016 & 0.03314 \\
\hline 4 & 6.91993 & 0.03025 & -6.10629 & 0.03881 \\
\hline 5 & 2.72234 & 0.03727 & -6.15793 & 0.03509 \\
\hline 6 & 6.28424 & 0.02797 & -2.67672 & 0.03531 \\
\hline 7 & 9.69902 & 0.03363 & -6.20311 & 0.03639 \\
\hline 8 & 7.53027 & 0.03454 & -9.40478 & 0.03526 \\
\hline 9 & 6.16785 & 0.03740 & -9.66629 & 0.03360 \\
\hline 10 & 3.81149 & 0.04187 & -3.70895 & 0.03210 \\
\hline 11 & 3.24031 & 0.03945 & -6.20244 & 0.03460 \\
\hline 12 & 6.16889 & 0.03365 & -9.22321 & 0.03341 \\
\hline 13 & 9.19902 & 0.03092 & -6.25909 & 0.03414 \\
\hline 14 & 6.28107 & 0.03176 & -3.24564 & 0.03556 \\
\hline 15 & 6.22443 & 0.03142 & -5.56342 & 0.02990 \\
\hline 16 & 6.23718 & 0.03893 & -5.41367 & 0.03784 \\
\hline
\end{tabular}


APPENDIX A. CALIBRATION COEFFICIENTS FOR VARIOUS MULTI-POSITION ANALYSES USED TO DETERMINE NEGLIGIBLE COEFFICIENT TERMS

as the angles between the torquer axes on the GyroWheel ${ }^{\mathrm{TM}}$ and the GyroWheel ${ }^{\mathrm{TM}}$ rate input axes. $\alpha, \beta$, and $\gamma$ when discussing ground calibration through multiposition testing are defined as the misalignment angles between the respective $\mathrm{X}$, $\mathrm{Y}$, and $\mathrm{Z}$ table system axes and the GyroWheel ${ }^{\mathrm{TM}}$ rate input axes. In Table A.2, the subscript ' 1 ' is utilized to represent the misalignment angles as measured from the $\mathrm{X}$ rate input axis while subscript ' 2 ' is utilized to represent the misalignment angles as measured from the $\mathrm{Y}$ rate input axis. For example $\gamma_{2}$ is defined as the misalignment angle between the $\mathrm{Z}$ table axis and the GyroWheel ${ }^{\mathrm{TM}} \mathrm{Y}$ rate input axis. Theoretically $\alpha_{1}+\alpha_{2}$ should equal 90 degrees and $\beta_{1}+\beta_{2}$ should equal 90 degrees.

The calibration cocfficients calculated from the GyroWhcel ${ }^{\text {TM }}$ Command Data from a reduced data set forming a 13 position test (positions 1,2,3,4,5,6,7,8,9,10,11,12,15) are shown in Table A.3. The calibration coefficients calculated from the GyroWhcel TM Command Data from a different reduced data set forming a 13 position test (positions $1,2,3,4,5,6,7,8,9,10,13,14,15)$ are shown in Table A.4. It is interesting to note that both reduced data sets produce better results than the 15 position test in that the standard deviations are lower than those of the 15 position test. To four decimal places, the two 13 position results are identical. As well, $\alpha_{1}+\beta_{1}$ and $\alpha_{2}+\beta_{2}$ are equal to 90 degrees for both 13 position tests. This makes sense since the $\mathrm{X}$ and $\mathrm{Y}$ table axes are perfectly orthogonal. As well $\gamma_{1}$ and $\gamma_{2}$ are both equal to 90 degrees meaning that the $\mathrm{Z}$ table axis is perfectly orthogonal to the GyroWheel ${ }^{\mathrm{TM}} \mathrm{X}$ and $\mathrm{Y}$ rate input axes.

The calibration coefficients calculated from the GyroWhec ${ }^{\text {TM }}$ Command Data from a reduced data sct forming an 11 position test (positions $1,2,3,4,5,6,7,8,9,10,15$ ) are shown in Table A.5. The calibration coefficients calculated for 11 position testing are the same to four decimal places as those for 13 position testing.

The calibration coefficients calculated from the GyroWhecl ${ }^{\text {TM }}$ Command Data from a reduced data set forming an 8 position test (positions $1,2,3,4,5,6,7,8$ ) are 
APPENDIX A. CALIBRATION COEFFICIENTS FOR VARIOUS MULTI-POSITION ANALYSES USED TO DETERMINE NEGLIGIBLE COEFFICIENT TERMS

Table A.2: 15 position test (7 September 2002): calculated calibration coefficients

\begin{tabular}{|l|l|l|l|l|}
\hline \multicolumn{5}{|c|}{ GyroWheel TM } \\
\hline$k_{x}$ & $\sigma k_{x}$ & $k_{y}$ & $\sigma k_{y}$ & Units \\
9.933501194 & 1.1 & 14.79202446 & 0.62 & $\mathrm{deg} / \mathrm{hr} / \mathrm{mA}$ \\
\hline$B_{x}$ & $\sigma B_{x}$ & $B_{y}$ & $\sigma B_{y}$ & \\
-89.09515304 & 5.1 & 75.9464067 & 6.7 & $\mathrm{deg} / \mathrm{hr}$ \\
\hline$D(x)_{X}$ & $\sigma D(x)_{X}$ & $D(y)_{Y}$ & $\sigma D(y)_{Y}$ & \\
-43.19776727 & 1.9 & -33.13923205 & 1.5 & $\mathrm{deg} / \mathrm{hr} / \mathrm{g}$ \\
\hline$D(x)_{Y}$ & $\sigma D(x)_{Y}$ & $D(y)_{X}$ & $\sigma D(y)_{X}$ & \\
5.249594452 & 0.99 & 0.147984741 & 0.37 & $\mathrm{deg} / \mathrm{hr} / \mathrm{g}$ \\
\hline$D(x)_{Z}$ & $\sigma D(x)_{Z}$ & $D(y)_{Z}$ & $\sigma D(y)_{Z}$ & \\
-3.276947868 & 1.8 & -8.15686779 & 0.55 & $\mathrm{deg} / \mathrm{hr} / \mathrm{g}$ \\
\hline$D(x)_{X X}$ & $\sigma D(x)_{X X}$ & $D(y)_{Y Y}$ & $\sigma D(y)_{Y Y}$ & \\
1.700080495 & 0.51 & -13.07635034 & 1.7 & $\mathrm{deg} / \mathrm{hr} / \mathrm{g}^{2}$ \\
\hline$D(x)_{Z Z}$ & $\sigma D(x)_{Z Z}$ & $D(y)_{Z Z}$ & $\sigma D(y)_{Z Z}$ & \\
-2.4144969 & 1.9 & -14.10294661 & 0.37 & $\mathrm{deg} / \mathrm{hr} / \mathrm{g}^{2}$ \\
\hline$D(x)_{X Y}$ & $\sigma D(x)_{X Y}$ & $D(y)_{X Y}$ & $\sigma D(y)_{X Y}$ & \\
26.72316712 & 3.4 & 48.80934385 & 3.1 & $\mathrm{deg} / \mathrm{hr} / \mathrm{g}^{2}$ \\
\hline$D(x)_{X Z}$ & $\sigma D(x)_{X Z}$ & $D(y)_{X Z}$ & $\sigma D(y)_{X Z}$ & \\
0.935542566 & 0.98 & -1.264720757 & 0.65 & $\mathrm{deg} / \mathrm{hr} / \mathrm{g}^{2}$ \\
\hline$D(x)_{Y Z}$ & $\sigma D(x)_{Y Z}$ & $D(y)_{Y Z}$ & $\sigma D(y)_{Y Z}$ & \\
-5.992265454 & 1.7 & -13.8530638 & 1.3 & $\mathrm{deg} / \mathrm{hr} / \mathrm{g}^{2}$ \\
\hline$\alpha_{1}$ & $\sigma \alpha_{1}$ & $\alpha \alpha_{2}$ & $\sigma \alpha_{2}$ & \\
11.5157302 & 8.5 & 89.81835837 & 2.1 & $\mathrm{deg}$ \\
\hline$\beta_{1}$ & $\sigma \beta_{1}$ & $\beta_{2}$ & $\sigma \beta_{2}$ & \\
89.8473113 & 1.5 & 49.3166925 & 5.6 & $\mathrm{deg}$ \\
\hline$\gamma_{1}$ & $\sigma \gamma_{1}$ & $\gamma_{2}$ & $\sigma \gamma_{2}$ & \\
101.5142582 & 11 & 139.3162809 & 5.6 & $\mathrm{deg}$ \\
\hline & & & & \\
\hline & & & & \\
\hline
\end{tabular}


APPENDIX A. CALIBRATION COEFFICIENTS FOR VARIOUS MULTI-POSITION ANALYSES USED TO DETERMINE NEGLIGIBLE COEFFICIENT TERMS

Table A.3: Reduced 13(a) position test (7 September 2002): calculated calibration coefficients

\begin{tabular}{|c|c|c|c|c|}
\hline \multicolumn{4}{|c|}{ GyroWheel $^{\text {TM }}$ Command Data } & \multirow{2}{*}{$\begin{array}{l}\text { Units } \\
\mathrm{deg} / \mathrm{hr} / \mathrm{mA}\end{array}$} \\
\hline $\begin{array}{l}k_{x} \\
15.2382\end{array}$ & $\begin{array}{l}\sigma k_{x} \\
0.52\end{array}$ & $\begin{array}{l}k_{y} \\
15.0958\end{array}$ & $\begin{array}{l}\sigma k_{y} \\
0.52\end{array}$ & \\
\hline $\begin{array}{l}B_{x} \\
-93.3001\end{array}$ & $\begin{array}{l}\sigma B_{x} \\
3.2\end{array}$ & $\begin{array}{l}B_{y} \\
105.2539\end{array}$ & $\begin{array}{l}\sigma B_{y} \\
3.6\end{array}$ & $\mathrm{deg} / \mathrm{hr}$ \\
\hline $\begin{array}{l}D(x)_{X} \\
-43.1978\end{array}$ & $\begin{array}{l}\sigma D(x)_{X} \\
1.9\end{array}$ & $\begin{array}{l}D(y)_{Y} \\
-33.1392\end{array}$ & $\begin{array}{l}\sigma D(y)_{Y} \\
1.5\end{array}$ & $\mathrm{deg} / \mathrm{hr} / \mathrm{g}$ \\
\hline $\begin{array}{l}D(x)_{Y} \\
3.4924\end{array}$ & $\begin{array}{l}\sigma D(x)_{Y} \\
0.55\end{array}$ & $\begin{array}{l}D(y)_{X} \\
0.2224\end{array}$ & $\begin{array}{l}\sigma D(y)_{X} \\
0.52\end{array}$ & $\mathrm{deg} / \mathrm{hr} / \mathrm{g}$ \\
\hline $\begin{array}{l}D(x)_{Z} \\
-1.1641\end{array}$ & $\begin{array}{l}\sigma D(x)_{Z} \\
0.30\end{array}$ & $\begin{array}{l}D(y)_{z} \\
-0.0633\end{array}$ & $\begin{array}{l}\sigma D(y)_{Z} \\
0.35\end{array}$ & $\operatorname{deg} / \mathrm{hr} / \mathrm{g}$ \\
\hline $\begin{array}{l}D(x)_{X X} \\
2.1100\end{array}$ & $\begin{array}{l}\sigma D(x)_{X X} \\
0.54\end{array}$ & $\begin{array}{c}D(y)_{Y Y} \\
-10.6148 \\
\end{array}$ & $\begin{array}{l}\sigma D(y)_{Y Y} \\
0.63\end{array}$ & $\operatorname{deg} / \mathrm{hr} / \mathrm{g}^{2}$ \\
\hline $\begin{array}{c}D(x)_{Z Z} \\
-0.0890 \\
\end{array}$ & $\begin{array}{l}\sigma D(x)_{Z Z} \\
0.49 \\
\end{array}$ & $\begin{array}{c}D(y)_{Z Z} \\
-10.3850 \\
\end{array}$ & $\begin{array}{l}\sigma D(y)_{Z Z} \\
0.59\end{array}$ & $\mathrm{deg} / \mathrm{hr} / \mathrm{g}^{2}$ \\
\hline $\begin{array}{l}D(x)_{X Y} \\
17.4204\end{array}$ & $\begin{array}{l}\sigma D(x)_{X Y} \\
1.1\end{array}$ & $\begin{array}{l}D(y)_{X Y} \\
47.8271\end{array}$ & $\begin{array}{l}\sigma D(y)_{X Y} \\
1.9\end{array}$ & $\operatorname{deg} / \mathrm{hr} / \mathrm{g}^{2}$ \\
\hline $\begin{array}{l}\alpha_{1} \\
0.1854 \\
\end{array}$ & $\begin{array}{l}\sigma \alpha_{1} \\
1.3 \\
\end{array}$ & $\begin{array}{l}\alpha_{2} \\
89.7658 \\
\end{array}$ & $\begin{array}{l}\sigma \alpha_{2} \\
2.3\end{array}$ & $\operatorname{deg}$ \\
\hline $\begin{array}{l}\beta_{1} \\
89.8146 \\
\end{array}$ & $\begin{array}{l}\sigma \beta_{1} \\
2.2 \\
\end{array}$ & $\begin{array}{l}\beta_{2} \\
0.2342\end{array}$ & $\begin{array}{l}\sigma \beta_{2} \\
1.4\end{array}$ & $\operatorname{deg}$ \\
\hline $\begin{array}{l}\gamma_{1} \\
90.0000\end{array}$ & $\begin{array}{l}\sigma \gamma_{1} \\
0.00\end{array}$ & $\begin{array}{l}\gamma_{2} \\
90.0000\end{array}$ & $\begin{array}{l}\sigma \gamma_{2} \\
0.00\end{array}$ & $\operatorname{deg}$ \\
\hline
\end{tabular}


APPENDIX A. CALIBRATION COEFFICIENTS FOR VARIOUS

MULTI-POSITION ANALYSES USED TO DETERMINE NEGLIGIBLE

COEFFICIENT TERMS

Table A.4: Reduced 13(b) position test (7 September 2002): calculated calibration coefficients

\begin{tabular}{|c|c|c|c|c|}
\hline \multicolumn{4}{|c|}{ GyroWheel $^{\text {TM }}$ Command Data } & Units \\
\hline $\begin{array}{l}k_{x} \\
15.2382\end{array}$ & $\begin{array}{l}\sigma k_{x} \\
0.52 \\
\end{array}$ & $\begin{array}{l}k_{y} \\
15.0958 \\
\end{array}$ & $\begin{array}{l}\sigma k_{y} \\
0.52\end{array}$ & $\operatorname{deg} / \mathrm{hr} / \mathrm{mA}$ \\
\hline $\begin{array}{l}B_{x} \\
-93.3001\end{array}$ & $\begin{array}{l}\sigma B_{x} \\
3.2\end{array}$ & $\begin{array}{l}B_{y} \\
105.2539\end{array}$ & $\begin{array}{l}\sigma B_{y} \\
3.6\end{array}$ & $\operatorname{deg} / \mathrm{hr}$ \\
\hline $\begin{array}{l}D(x)_{X} \\
-43.1978\end{array}$ & $\begin{array}{l}\sigma D(x)_{X} \\
1.9\end{array}$ & $\begin{array}{l}D(y)_{Y} \\
-33.1392\end{array}$ & $\begin{array}{l}\sigma D(y)_{Y} \\
1.5\end{array}$ & $\mathrm{deg} / \mathrm{hr} / \mathrm{g}$ \\
\hline $\begin{array}{l}D(x)_{Y} \\
3.4924 \\
\end{array}$ & $\begin{array}{l}\sigma D(x)_{Y} \\
0.53\end{array}$ & $\begin{array}{l}D(y)_{X} \\
0.2224\end{array}$ & $\begin{array}{l}\sigma D(y)_{X} \\
0.52\end{array}$ & $\operatorname{deg} / \mathrm{hr} / \mathrm{g}$ \\
\hline $\begin{array}{l}D(x)_{Z} \\
-1.1641\end{array}$ & $\begin{array}{l}\sigma D(x)_{Z} \\
0.30\end{array}$ & $\begin{array}{l}D(y)_{Z} \\
-0.0633\end{array}$ & $\begin{array}{l}\sigma D(y)_{Z} \\
0.34\end{array}$ & $\mathrm{deg} / \mathrm{hr} / \mathrm{g}$ \\
\hline $\begin{array}{l}D(x)_{X X} \\
2.1100\end{array}$ & $\begin{array}{l}\sigma D(x)_{X X} \\
0.55\end{array}$ & $\begin{array}{l}D(y)_{Y Y} \\
-10.6148\end{array}$ & $\begin{array}{l}\sigma D(y)_{Y Y} \\
0.64\end{array}$ & $\operatorname{deg} / \mathrm{hr} / \mathrm{g}^{2}$ \\
\hline $\begin{array}{l}D(x)_{Z Z} \\
-0.0890\end{array}$ & $\begin{array}{l}\sigma D(x)_{Z Z} \\
0.50\end{array}$ & $\begin{array}{c}D(y)_{Z Z} \\
-10.3850 \\
\end{array}$ & $\begin{array}{l}\sigma D(y)_{Z Z} \\
0.60\end{array}$ & $\operatorname{deg} / \mathrm{hr} / \mathrm{g}^{2}$ \\
\hline $\begin{array}{l}D(x)_{X Y} \\
17.4204\end{array}$ & $\begin{array}{l}\sigma D(x)_{X Y} \\
1.1\end{array}$ & $\begin{array}{l}D(y)_{X Y} \\
47.8271 \\
\end{array}$ & $\begin{array}{l}\sigma D(y)_{X Y} \\
1.9\end{array}$ & $\operatorname{deg} / \mathrm{hr} / \mathrm{g}^{2}$ \\
\hline $\begin{array}{l}\alpha_{1} \\
0.1854\end{array}$ & $\begin{array}{l}\sigma \alpha_{1} \\
1.3\end{array}$ & $\begin{array}{l}\alpha_{2} \\
89.7658\end{array}$ & $\begin{array}{l}\sigma \alpha_{2} \\
2.3\end{array}$ & $\operatorname{deg}$ \\
\hline $\begin{array}{l}\beta_{1} \\
89.8146\end{array}$ & $\begin{array}{l}\sigma \beta_{1} \\
2.2\end{array}$ & $\begin{array}{l}\beta_{2} \\
0.2342\end{array}$ & $\begin{array}{l}\sigma \beta_{2} \\
1.4\end{array}$ & $\operatorname{deg}$ \\
\hline $\begin{array}{l}\gamma_{1} \\
90.0000\end{array}$ & $\begin{array}{l}\sigma \gamma_{1} \\
0.00\end{array}$ & $\begin{array}{l}\gamma_{2} \\
90.0000\end{array}$ & $\begin{array}{l}\sigma \gamma_{2} \\
0.00\end{array}$ & $\operatorname{deg}$ \\
\hline
\end{tabular}


APPENDIX A. CALIBRATION COEFFICIENTS FOR VARIOUS MULTI-POSITION ANALYSES USED TO DETERMINE NEGLIGIBLE COEFFICIENT TERMS

Table A.5: Reduced 11 position test (7 September 2002): calculated calibration coefficients

\begin{tabular}{|c|c|c|c|c|}
\hline \multicolumn{4}{|c|}{ GyroWheel $^{\text {TM }}$ Command Data } & \multirow{3}{*}{$\begin{array}{l}\text { Units } \\
\mathrm{deg} / \mathrm{hr} / \mathrm{mA}\end{array}$} \\
\hline$k_{x}$ & $\sigma k_{x}$ & $k_{y}$ & $\sigma k_{y}$ & \\
\hline 15.23816693 & 0.52 & 15.09582706 & 0.52 & \\
\hline$B_{x}$ & $\sigma B_{x}$ & $B_{y}$ & $\sigma B_{y}$ & \\
\hline-93.30005785 & 3.0 & 105.2539412 & 3.6 & $\mathrm{deg} / \mathrm{hr}$ \\
\hline$D(x)_{X}$ & $\sigma D(x)_{X}$ & $D(y)_{Y}$ & $\sigma D(y)_{Y}$ & \\
\hline-43.19776727 & 1.9 & -33.13923205 & 1.5 & $\mathrm{deg} / \mathrm{hr} / \mathrm{g}$ \\
\hline$D(x)_{Y}$ & $\sigma D(x)_{Y}$ & $D(y)_{X}$ & $\sigma D(y)_{X}$ & \\
\hline 3.492405559 & 0.53 & 0.222442628 & 0.53 & $\mathrm{deg} / \mathrm{hr} / \mathrm{g}$ \\
\hline$D(x)_{Z}$ & $\sigma D(x)_{Z}$ & $D(y)_{Z}$ & $\sigma D(y)_{Z}$ & \\
\hline-1.164113781 & 0.29 & -0.063305394 & 0.36 & $\mathrm{deg} / \mathrm{hr} / \mathrm{g}$ \\
\hline$D(x)_{X X}$ & $\sigma D(x)_{X X}$ & $D(y)_{Y Y}$ & $\sigma D(y)_{Y Y}$ & \\
\hline 2.109987251 & 0.53 & -10.61476613 & 0.63 & $\mathrm{deg} / \mathrm{hr} / \mathrm{g}^{2}$ \\
\hline$D(x)_{Z Z}$ & $\sigma D(x)_{Z Z}$ & $D(y)_{Z Z}$ & $\sigma D(y)_{Z Z}$ & \\
\hline-0.089042045 & 0.49 & -10.38497712 & 0.59 & $\mathrm{deg} / \mathrm{hr} / \mathrm{g}^{2}$ \\
\hline$D(x)_{X Y}$ & $\sigma D(x)_{X Y}$ & $D(y)_{X Y}$ & $\sigma D(y)_{X Y}$ & \\
\hline 17.42037699 & 1.06 & 47.82705878 & 1.9 & $\mathrm{deg} / \mathrm{hr} / \mathrm{g}^{2}$ \\
\hline$\alpha_{1}$ & $\sigma \alpha_{1}$ & $\alpha_{2}$ & $\sigma \alpha_{2}$ & \\
\hline 0.185372246 & 1.3 & 89.76577245 & 2.3 & deg \\
\hline$\beta_{1}$ & $\sigma \beta_{1}$ & $\beta_{2}$ & $\sigma \beta_{2}$ & \\
\hline 89.81462775 & 2.2 & 0.234227548 & 1.4 & deg \\
\hline
\end{tabular}


APPENDIX A. CALIBRATION COEFFICIENTS FOR VARIOUS MULTI-POSITION ANALYSES USED TO DETERMINE NEGLIGIBLE COEFFICIENT TERMS

Table A.6: Reduced 8 position test (7 September 2002): calculated calibration coefficients

\begin{tabular}{|l|l|l|l|l|}
\hline \multicolumn{4}{|c|}{ GyroWheel ${ }^{\text {TM }}$ Command Data } & Units \\
\hline \hline$k_{x}$ & $\sigma k_{x}$ & $k_{y}$ & $\sigma k_{y}$ & \\
15.23816693 & 0.52 & 15.09582706 & 0.52 & $\mathrm{deg} / \mathrm{hr} / \mathrm{mA}$ \\
\hline$B_{x}$ & $\sigma B_{x}$ & $B_{y}$ & $\sigma B_{y}$ & \\
-92.24507527 & 3.0 & 99.94664687 & 3.4 & $\mathrm{deg} / \mathrm{hr}$ \\
\hline$D(x)_{X}$ & $\sigma D(x)_{X}$ & $D(y)_{Y}$ & $\sigma D(y)_{Y}$ & \\
-40.08098096 & 1.8 & -42.45426382 & 1.9 & $\mathrm{deg} / \mathrm{hr} / \mathrm{g}$ \\
\hline$D(x)_{Y}$ & $\sigma D(x)_{Y}$ & $D(y)_{X}$ & $\sigma D(y)_{X}$ & \\
0.375609511 & 0.56 & 9.53744533 & 0.63 & $\mathrm{deg} / \mathrm{hr} / \mathrm{g}$ \\
\hline$D(x)_{Z}$ & $\sigma D(x)_{Z}$ & $D(y)_{Z}$ & $\sigma D(y)_{Z}$ & \\
-0.020089154 & 0.38 & 5.014377358 & 0.39 & $\mathrm{deg} / \mathrm{hr} / \mathrm{g}$ \\
\hline$\alpha_{1}$ & $\sigma \alpha_{1}$ & $\alpha_{2}$ & $\sigma \alpha_{2}$ & \\
0.185372245 & 1.3 & 89.76577245 & 2.3 & $\mathrm{deg}$ \\
\hline$\beta_{1}$ & $\sigma \beta_{1}$ & $\beta_{2}$ & $\sigma \beta_{2}$ & \\
89.81462775 & 2.2 & 0.234227548 & 1.4 & $\mathrm{deg}$ \\
\hline
\end{tabular}

shown in Table A.6. The calibration coefficients calculated for 8 position testing are different from those for 11 position testing. Only the scale factors, $k_{x}$ and $k_{y}$, and the misalignment angles remained unchanged. 


\section{Appendix B}

\section{Set of Current Plots Versus \\ Temperature for Combined \\ Temperature and Tilt Sweep}

\section{Testing}

The following data presented in Figures B.1 through B.8 was collected for the first eight positions of an eleven position multi-position test, where $\omega_{z}=130 \mathrm{rad} / \mathrm{s}$; and rotor tilt: $\phi_{x}=3^{\circ}$ and $\phi_{y}=0^{\circ}$. The graph of $i_{x}$ versus $T_{h, y}$ shows the original data scatter; the extrapolated cooling sweep line; the extrapolated heating sweep line; and their average, for each of the first cight positions. 


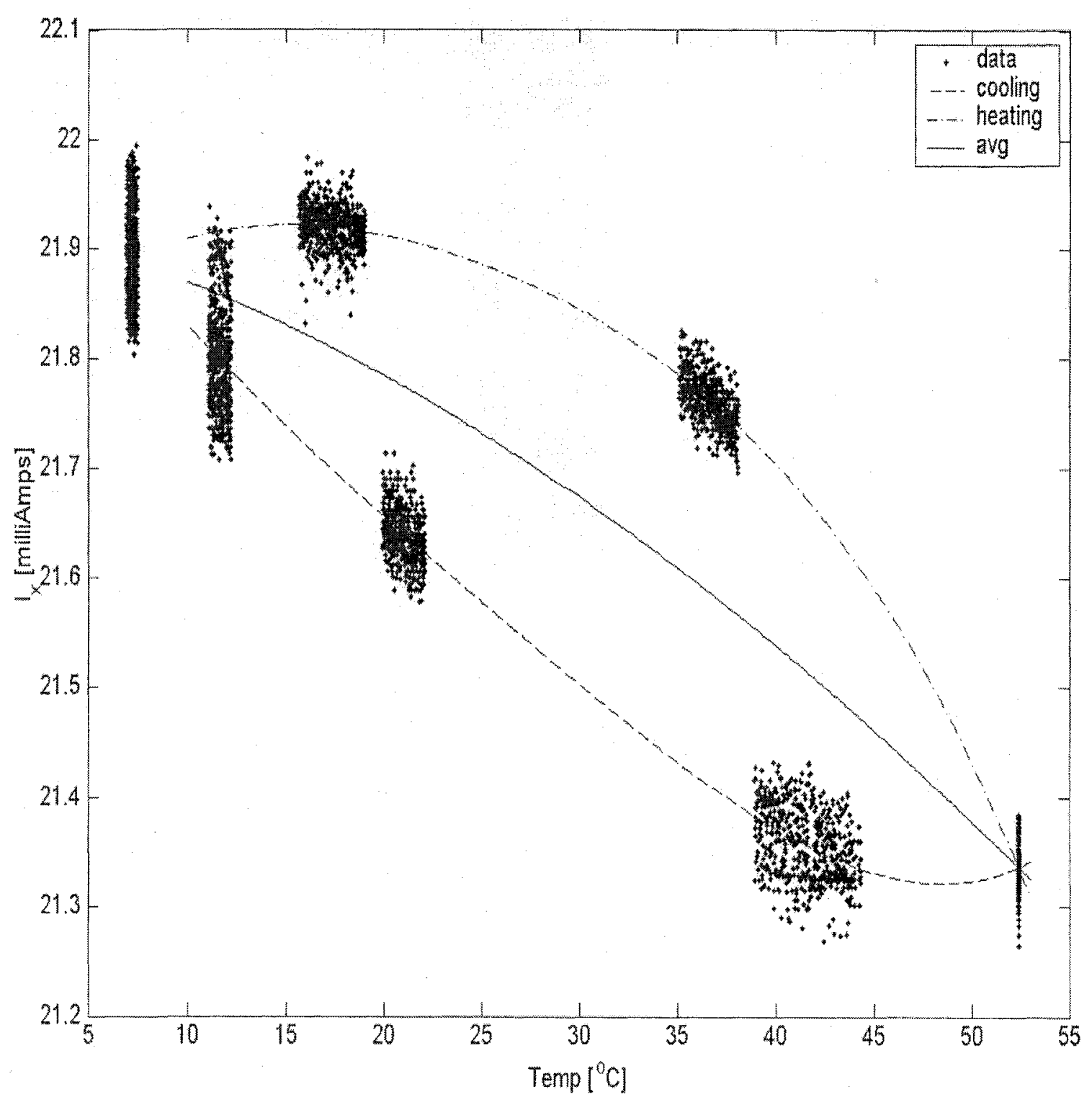

Figure B.1: Plot of data for $i_{x}$ versus $T_{h, y}$ for Multi-Position Test $1 ; \omega_{z}=130 \mathrm{rad} / \mathrm{s}$; $\phi_{x}=3^{\circ}$ and $\phi_{y}=0^{\circ}$ 


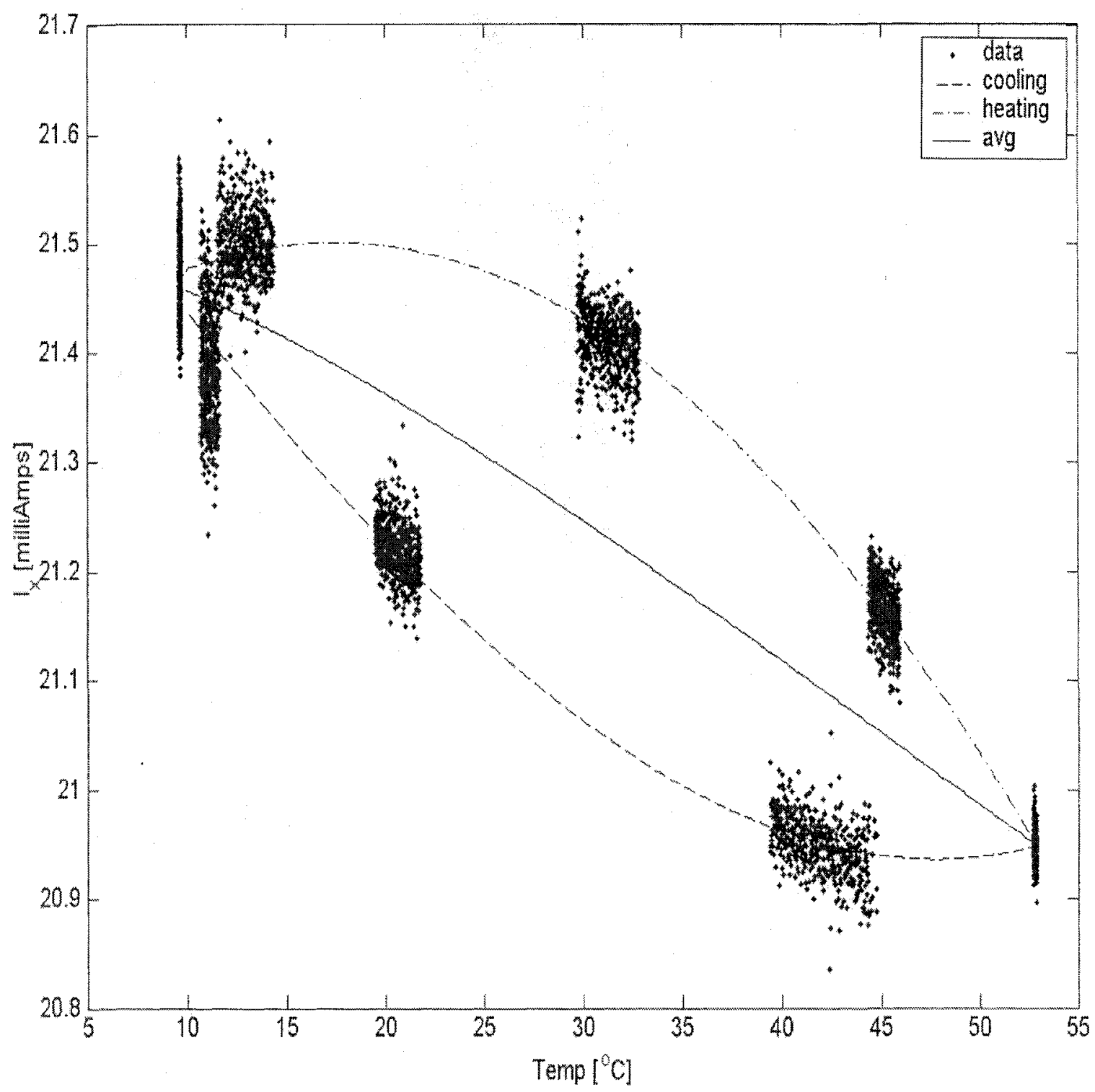

Figure B.2: Plot of data for $i_{x}$ versus $T_{h, y}$ for Multi-Position Test $2 ; \omega_{z}=130 \mathrm{rad} / \mathrm{s}$; $\phi_{x}=3^{\circ}$ and $\phi_{y}=0^{\circ}$ 


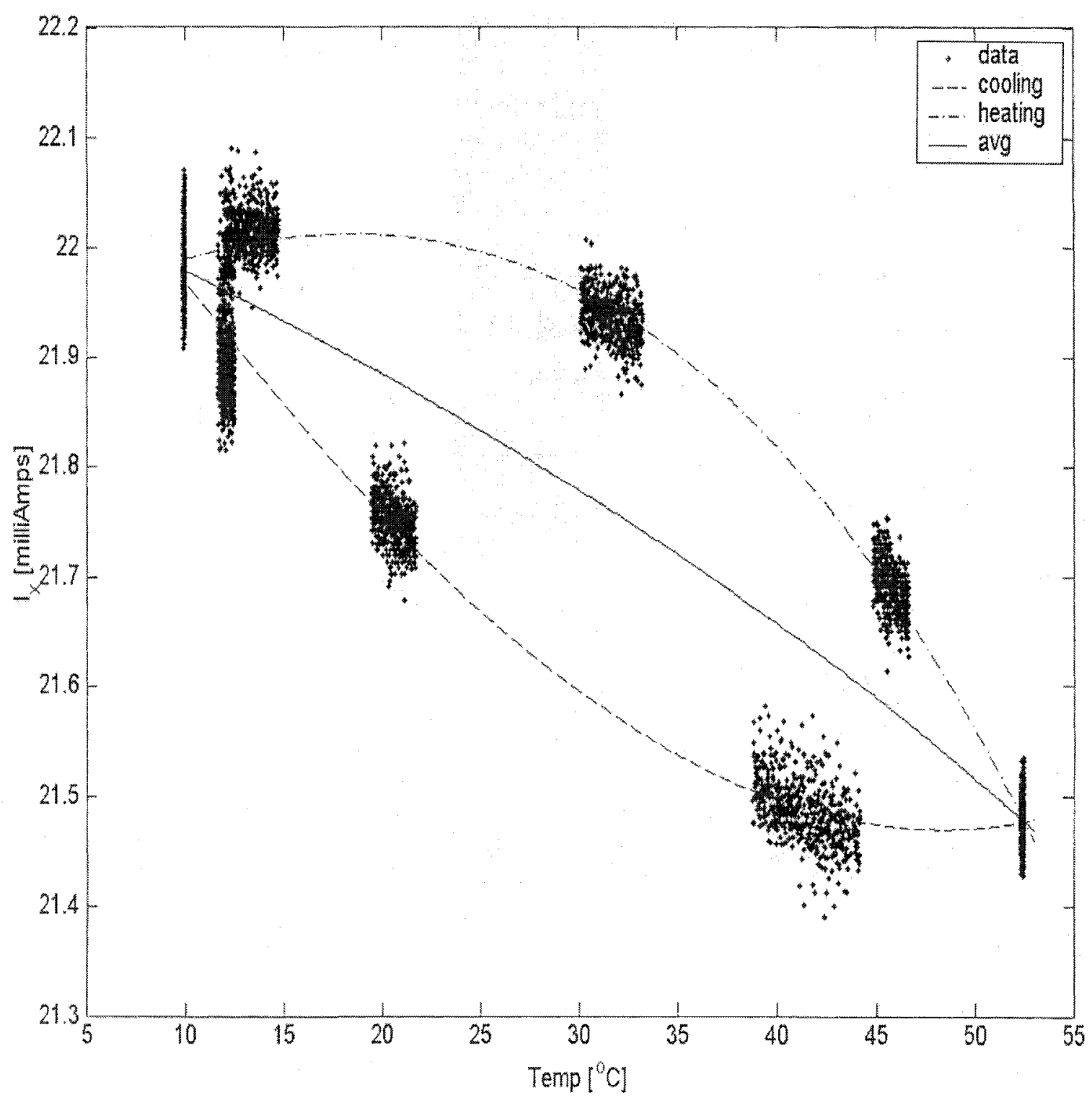

Figure B.3: Plot of data for $i_{x}$ versus $T_{h, y}$ for Multi-Position Test $3 ; \omega_{z}=130 \mathrm{rad} / \mathrm{s}$; $\phi_{x}=3^{\circ}$ and $\phi_{y}=0^{\circ}$ 


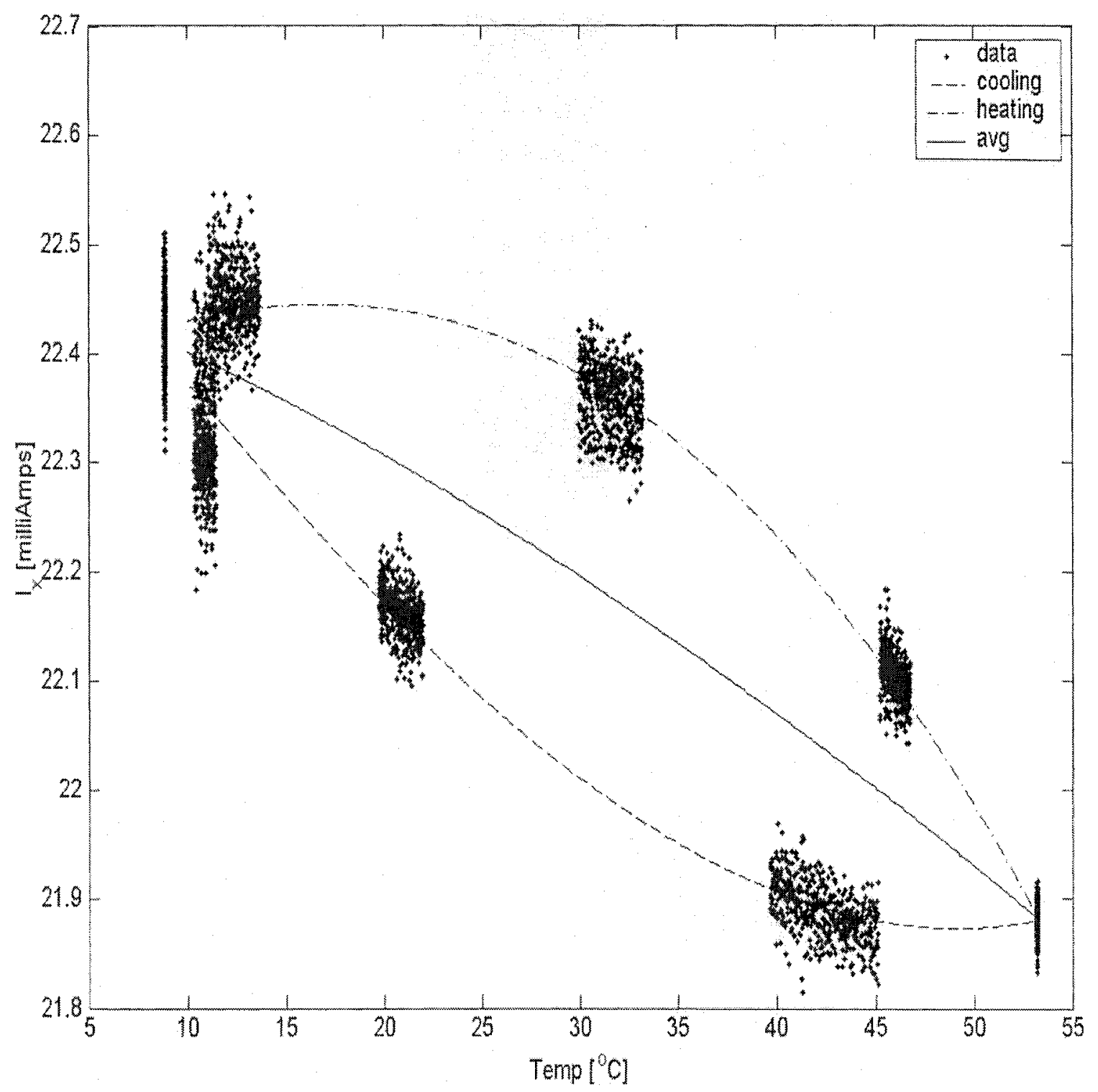

Figure B.4: Plot of data for $i_{x}$ versus $T_{h, y}$ for Multi-Position Test $4 ; \omega_{z}=130 \mathrm{rad} / \mathrm{s}$; $\phi_{x}=3^{\circ}$ and $\phi_{y}=0^{\circ}$ 


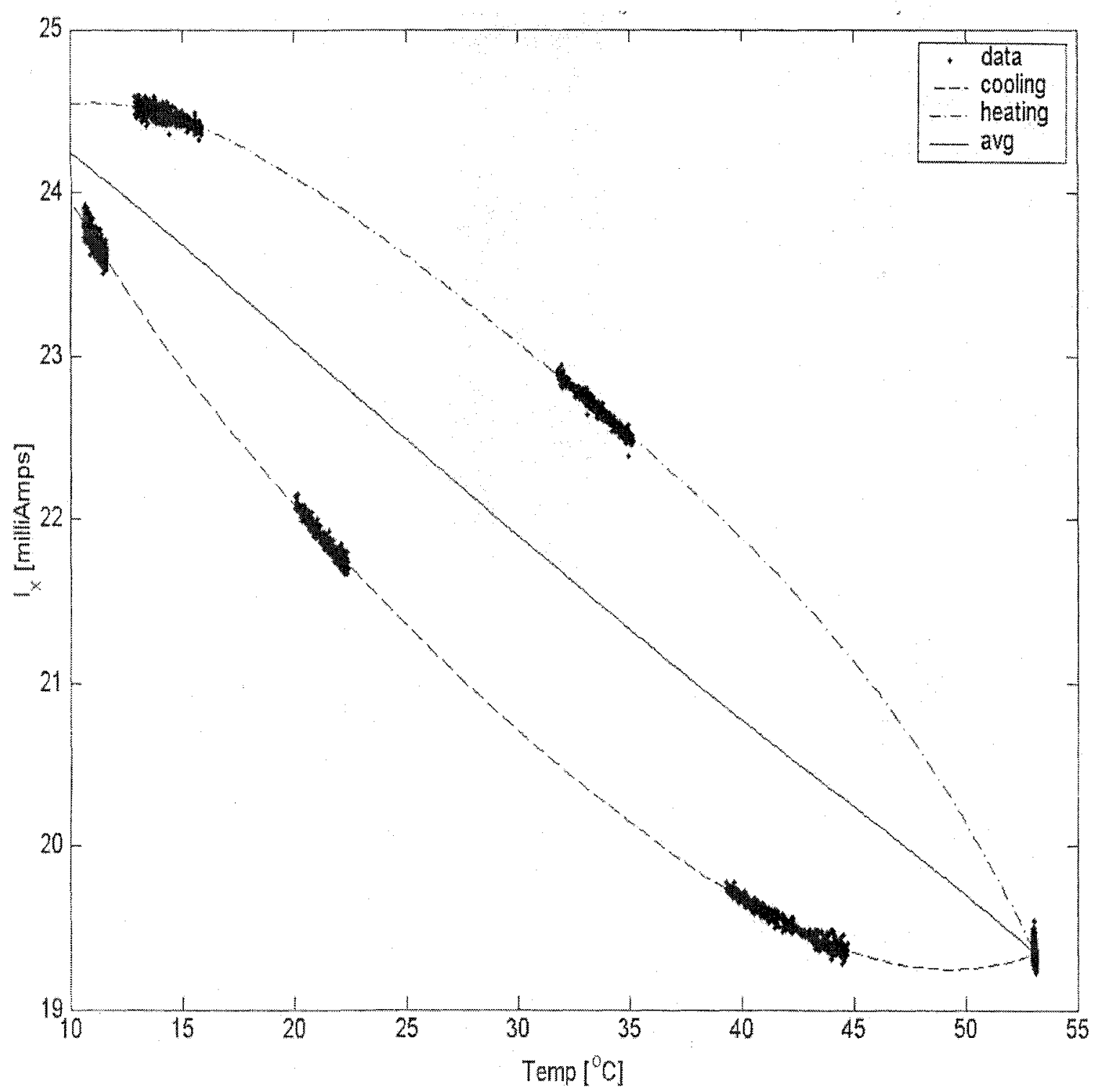

Figure B.5: Plot of data for $i_{x}$ versus $T_{h, y}$ for Multi-Position Test $5 ; \omega_{z}=130 \mathrm{rad} / \mathrm{s}$; $\phi_{x}=3^{\circ}$ and $\phi_{y}=0^{\circ}$ 


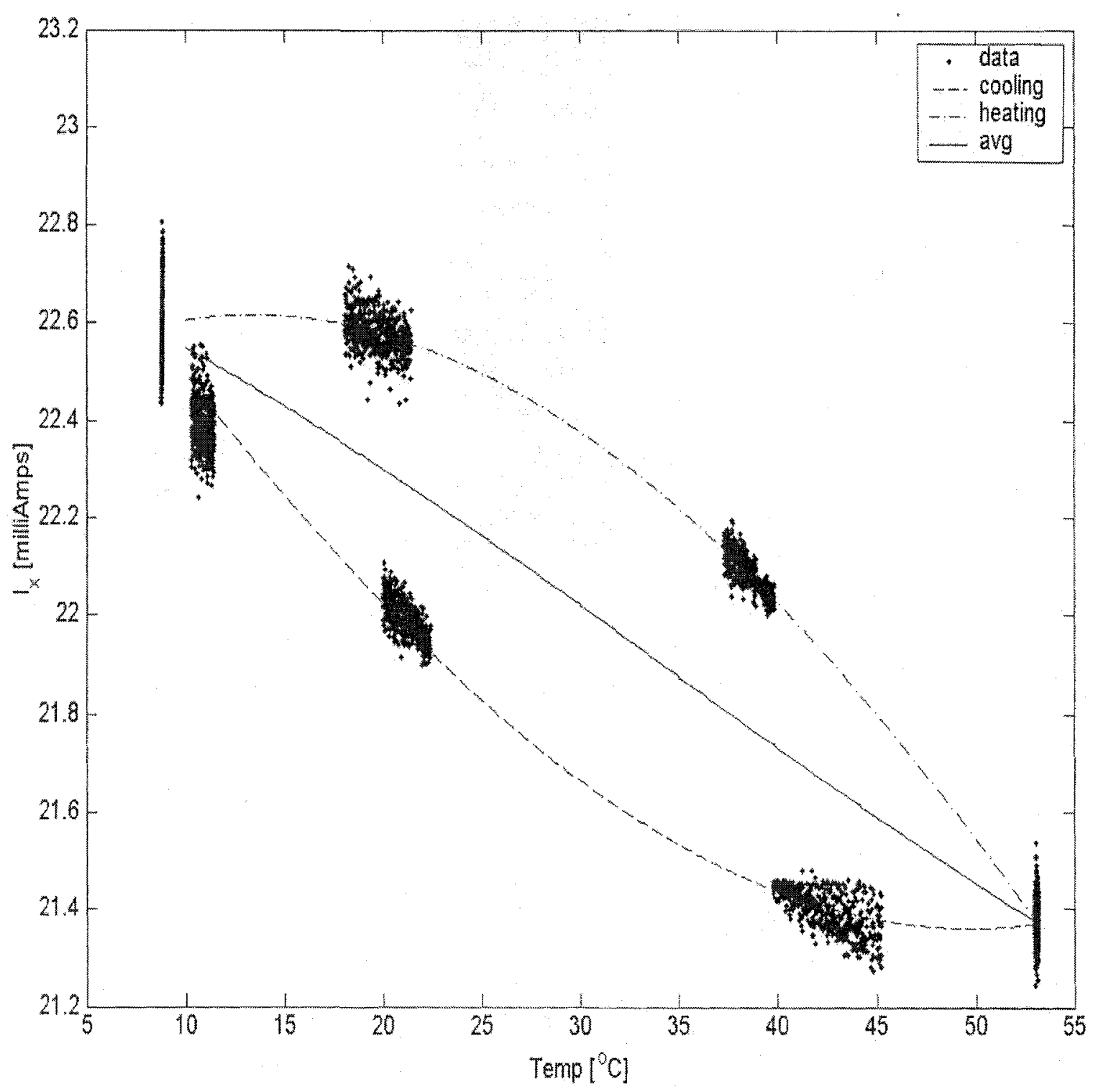

Figure B.6: Plot of data for $i_{x}$ versus $T_{h, y}$ for Multi-Position Test $6 ; \omega_{z}=130 \mathrm{rad} / \mathrm{s}$; $\phi_{x}=3^{\circ}$ and $\phi_{y}=0^{\circ}$ 


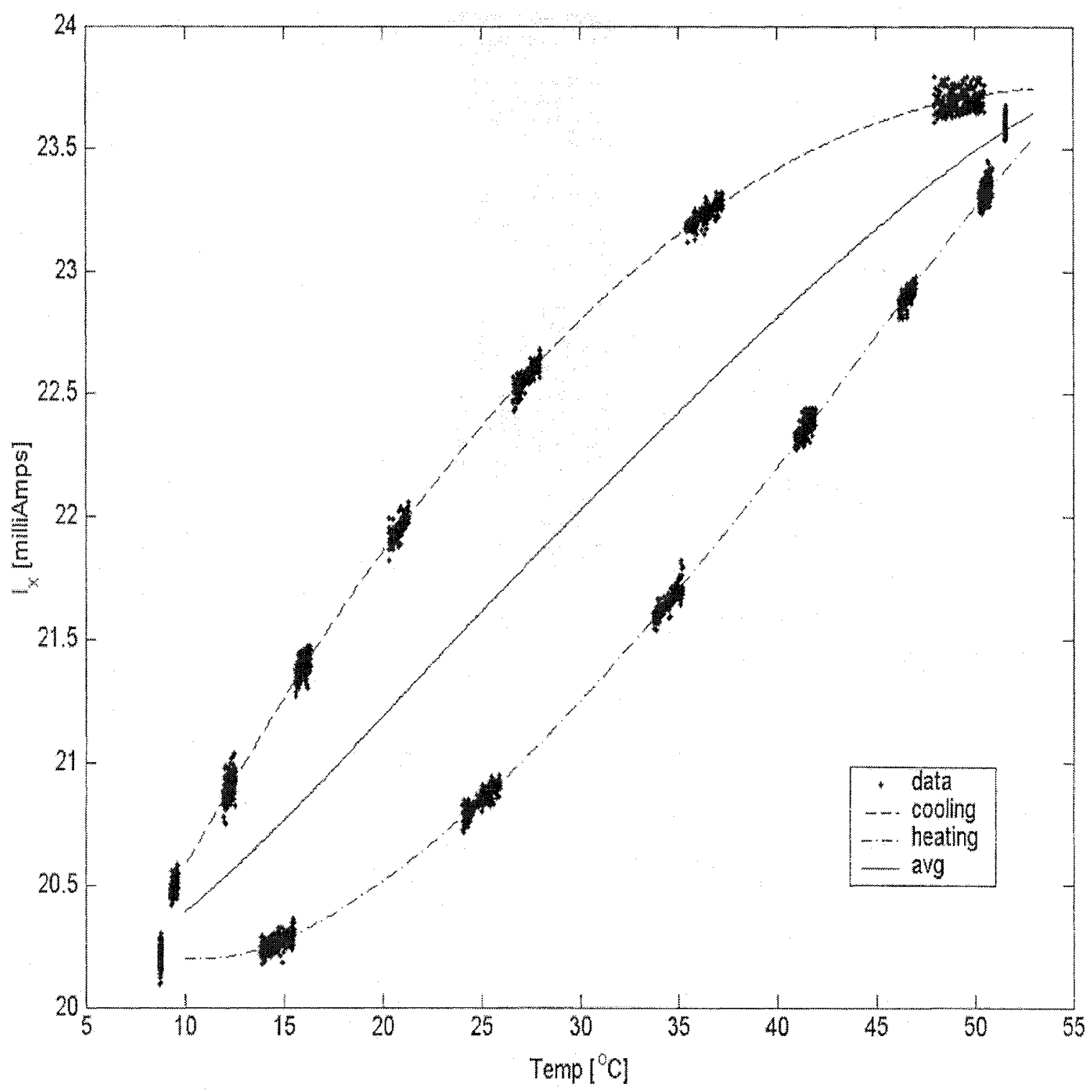

Figure B.7: Plot of data for $i_{x}$ versus $T_{h, y}$ for Multi-Position Test $7 ; \omega_{z}=130 \mathrm{rad} / \mathrm{s}$; $\phi_{x}=3^{\circ}$ and $\phi_{y}=0^{\circ}$ 


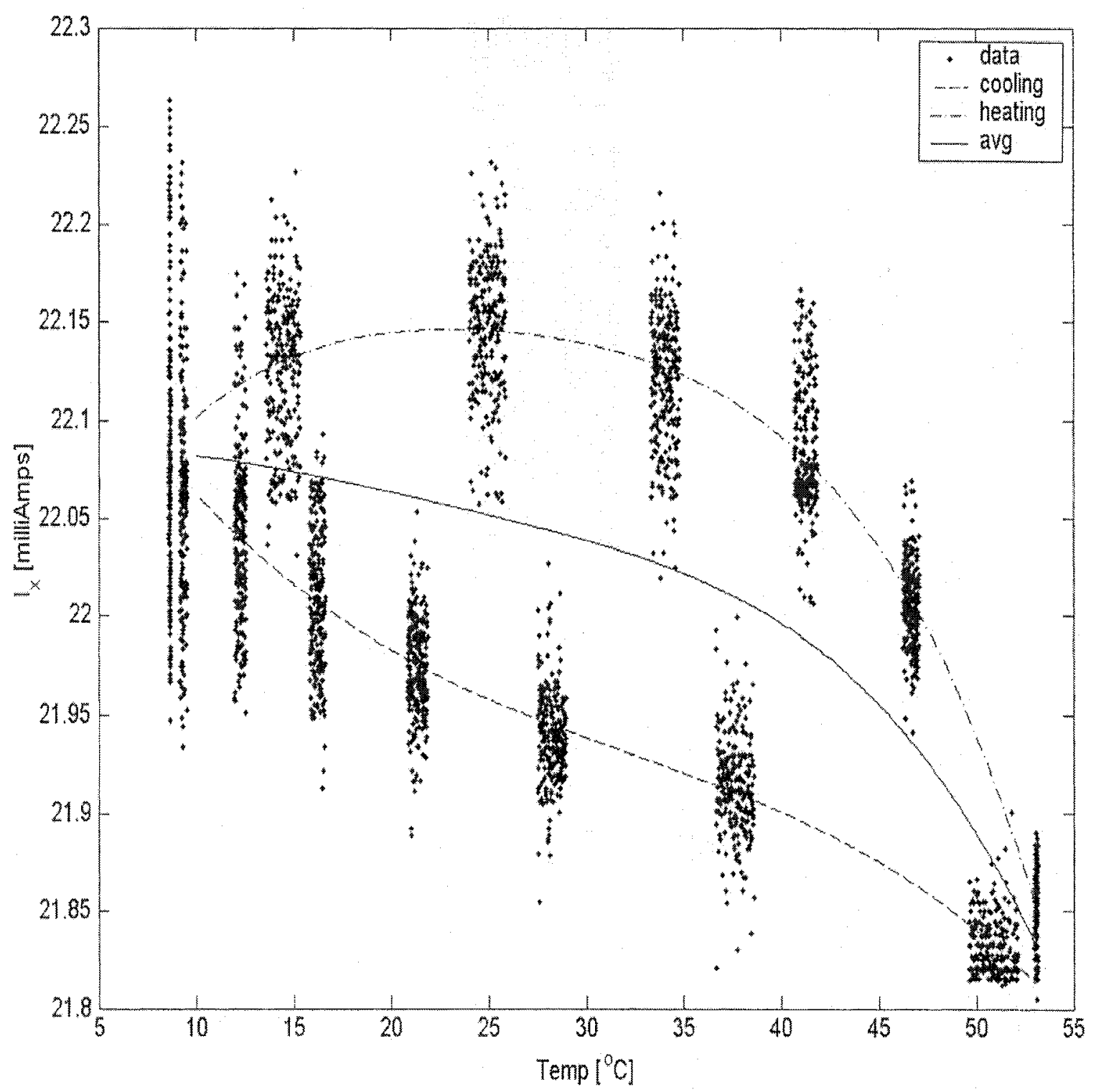

Figure B.8: Plot of data for $i_{x}$ versus $T_{h, y}$ for Multi-Position Test $8 ; \omega_{z}=130 \mathrm{rad} / \mathrm{s}$; $\phi_{x}=3^{\circ}$ and $\phi_{y}=0^{\circ}$ 


\section{Appendix C}

\section{Calculated Calibration Coefficients at Null Tilt Conditions}

The following data presented in Figures C.1 through C.8 was calculated from complete eleven position multi-position tests for each of the three wheel speeds: $\omega_{z}=130$ $\mathrm{rad} / \mathrm{s} ; \omega_{z}=153 \mathrm{rad} / \mathrm{s} ;$ and $\omega_{z}=176 \mathrm{rad} / \mathrm{s}$. The rotor tilt was at the null tilt condition: $\phi_{x}=0^{\circ}$ and $\phi_{y}=0^{\circ}$. The graphs show the calculated calibration coefficients $B_{x}^{\prime}, B_{y}^{\prime}, k_{x}^{\prime}, k_{y}^{\prime}, c_{g, x}^{\prime}, c_{g, y}^{\prime}, K_{\mathrm{dyn}, x}^{\prime}$, and $K_{\mathrm{dyn}, y}^{\prime}$ versus temperature. 


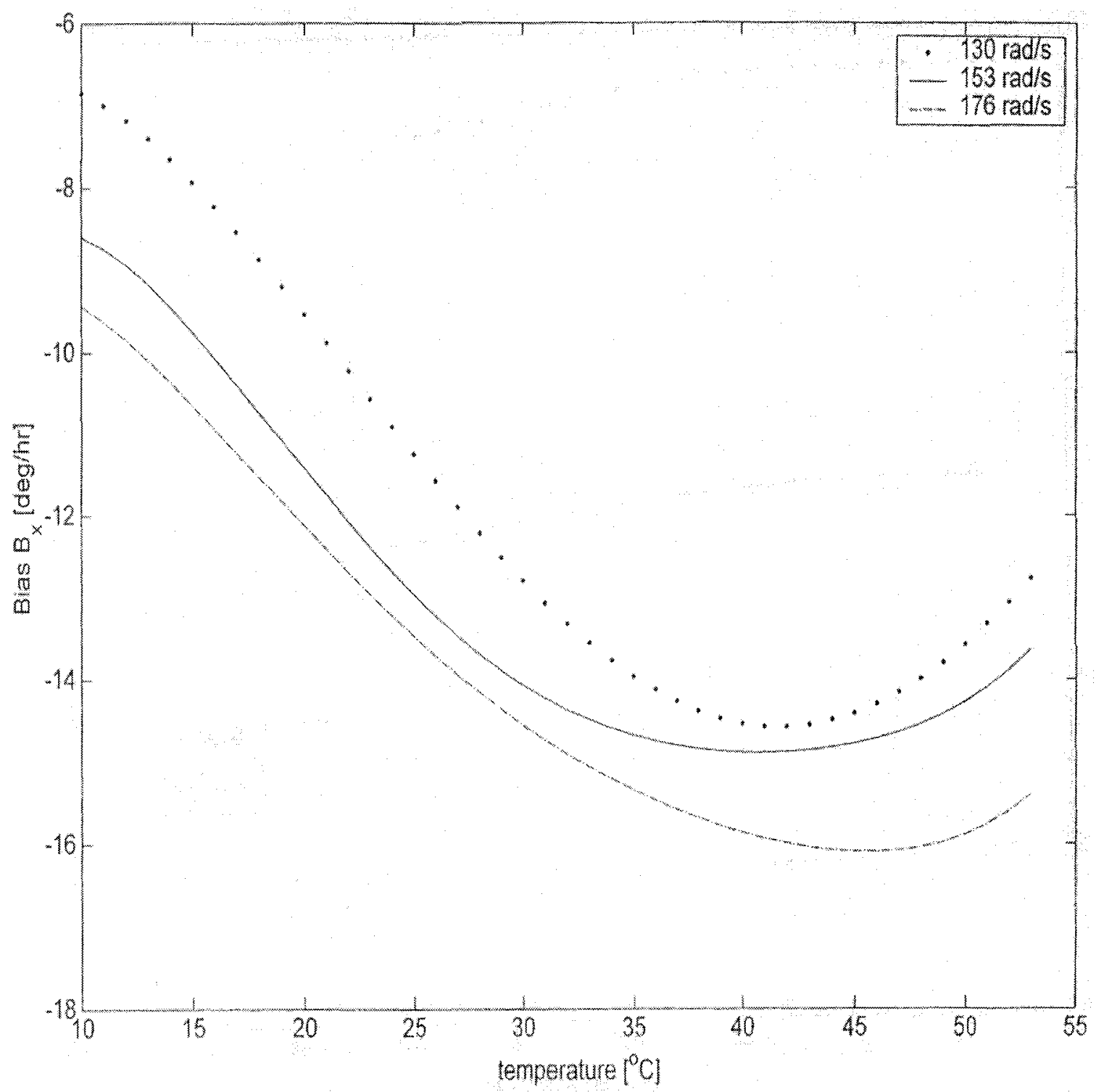

Figure C.1: Plot of calibrated value for $B_{x}^{\prime}$ versus $T_{h, y}$ for $\phi_{x}=0^{\circ}$ and $\phi_{y}=0^{\circ}$; $\omega_{z}=130 \mathrm{rad} / \mathrm{s}, 153 \mathrm{rad} / \mathrm{s}$, and $176 \mathrm{rad} / \mathrm{s}$. 


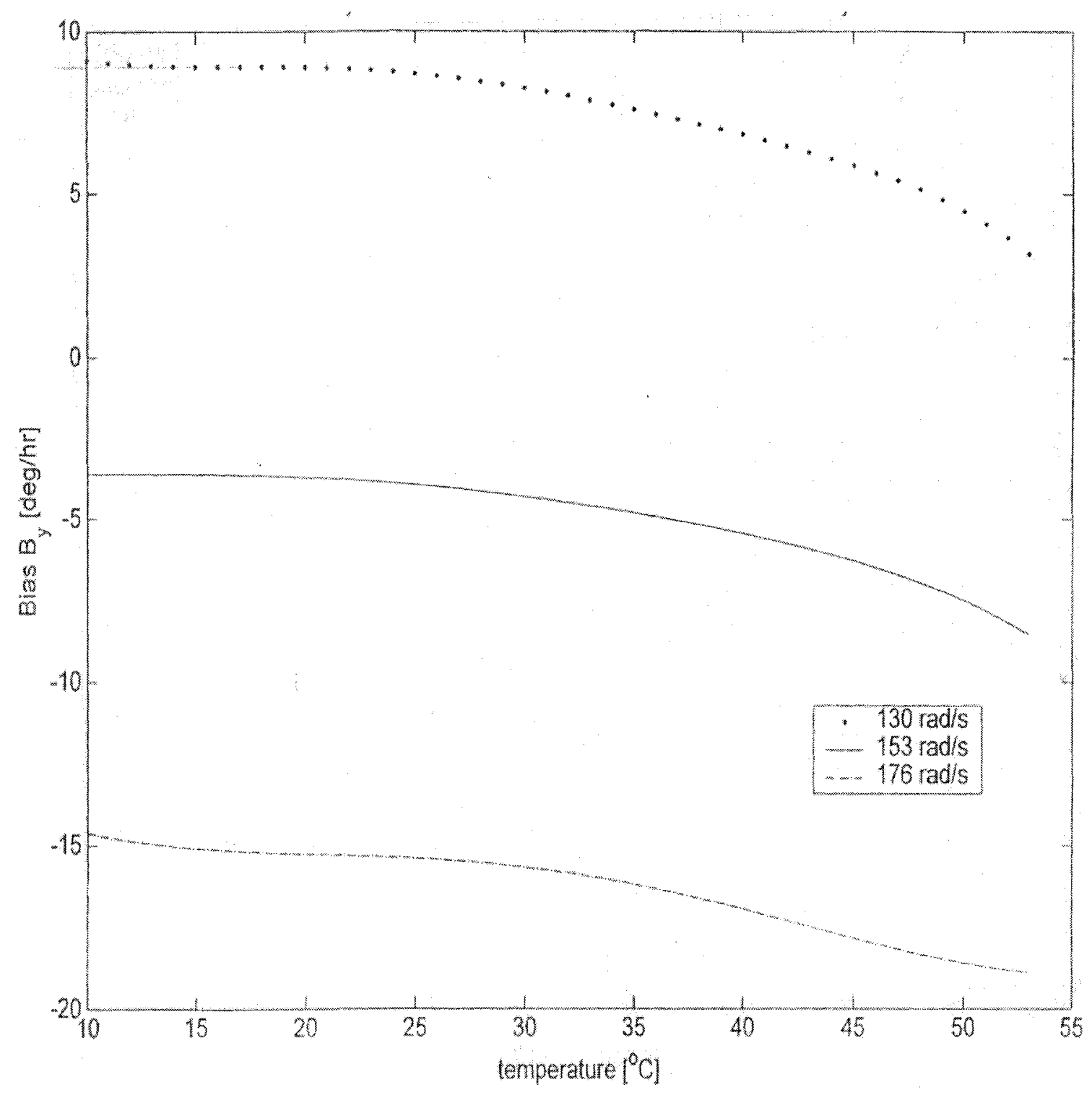

Figure C.2: Plot of calibrated value for $B_{y}^{\prime}$ versus $T_{h, x}$ for $\phi_{x}=0^{\circ}$ and $\phi_{y}=0^{\circ}$; $\omega_{z}=130 \mathrm{rad} / \mathrm{s}, 153 \mathrm{rad} / \mathrm{s}$, and $176 \mathrm{rad} / \mathrm{s}$. 


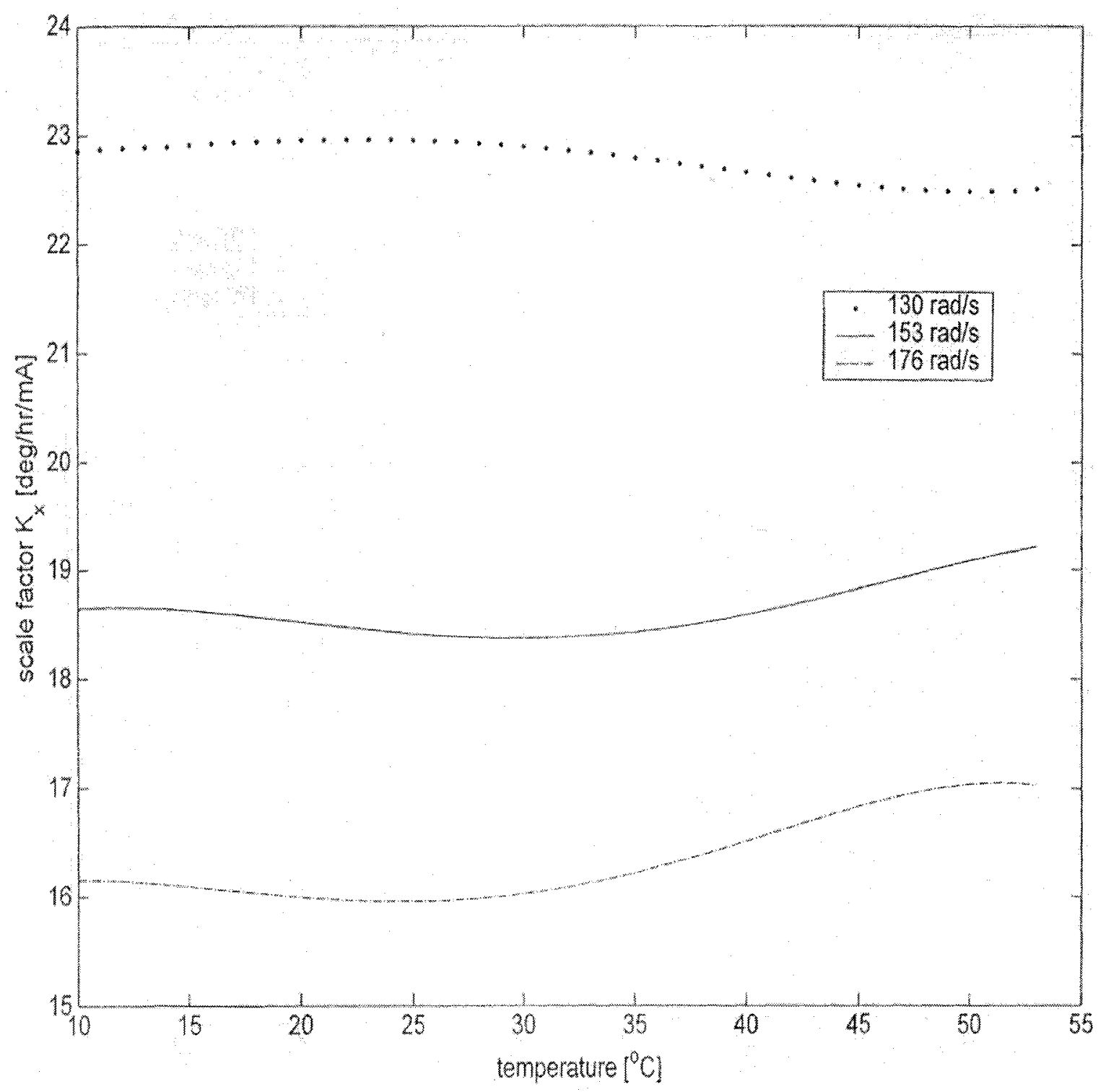

Figure C.3: Plot of calibrated value for $k_{x}^{\prime}$ versus $T_{h, y}$ for $\phi_{x}=0^{o}$ and $\phi_{y}=0^{o}$; $\omega_{z}=130 \mathrm{rad} / \mathrm{s}, 153 \mathrm{rad} / \mathrm{s}$, and $176 \mathrm{rad} / \mathrm{s}$. 


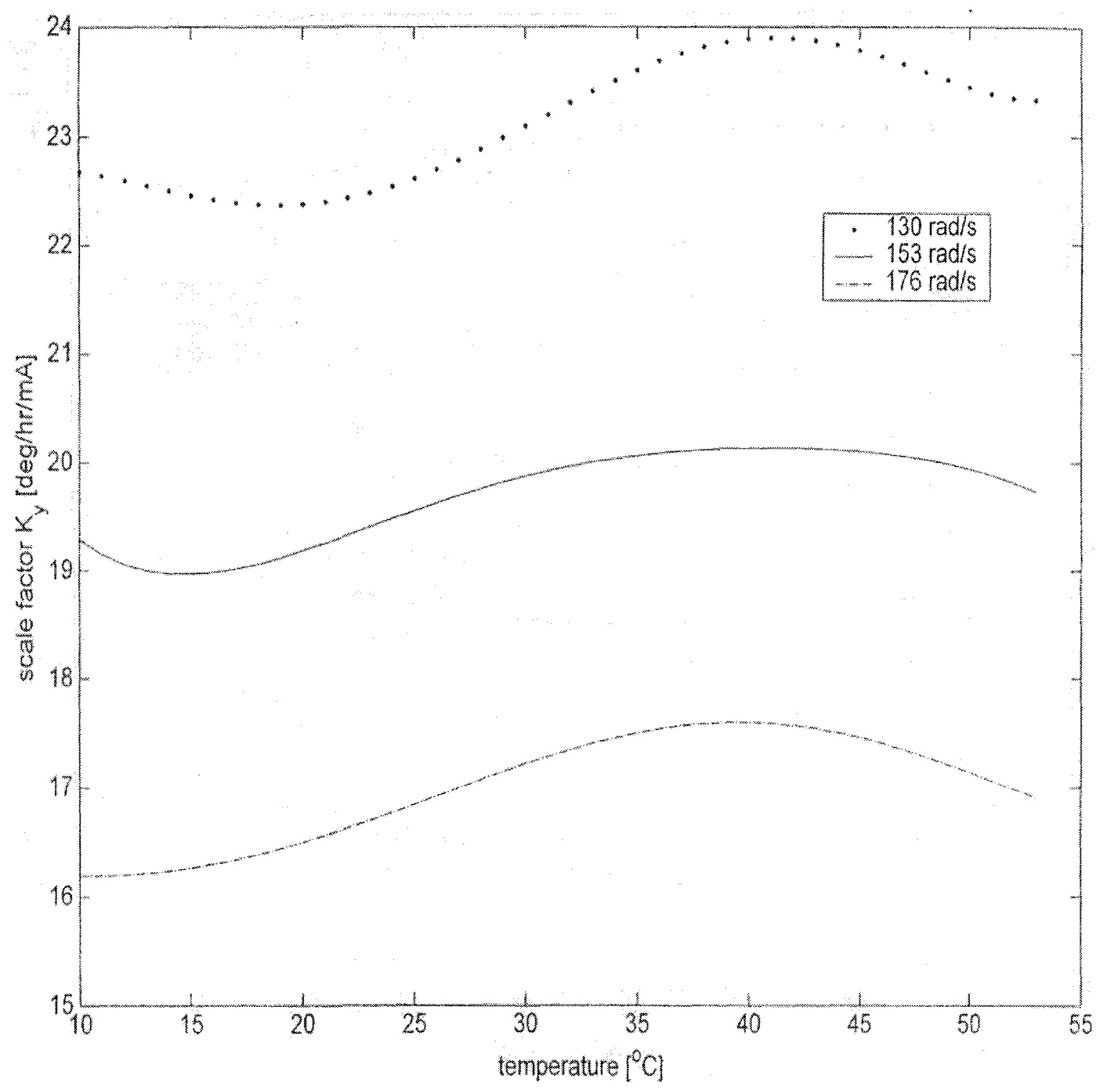

Figure C.4: Plot of calibrated value for $k_{y}^{\prime}$ versus $T_{h, x}$ for $\phi_{x}=0^{\circ}$ and $\phi_{y}=0^{\circ}$; $\omega_{z}=130 \mathrm{rad} / \mathrm{s}, 153 \mathrm{rad} / \mathrm{s}$, and $176 \mathrm{rad} / \mathrm{s}$. 


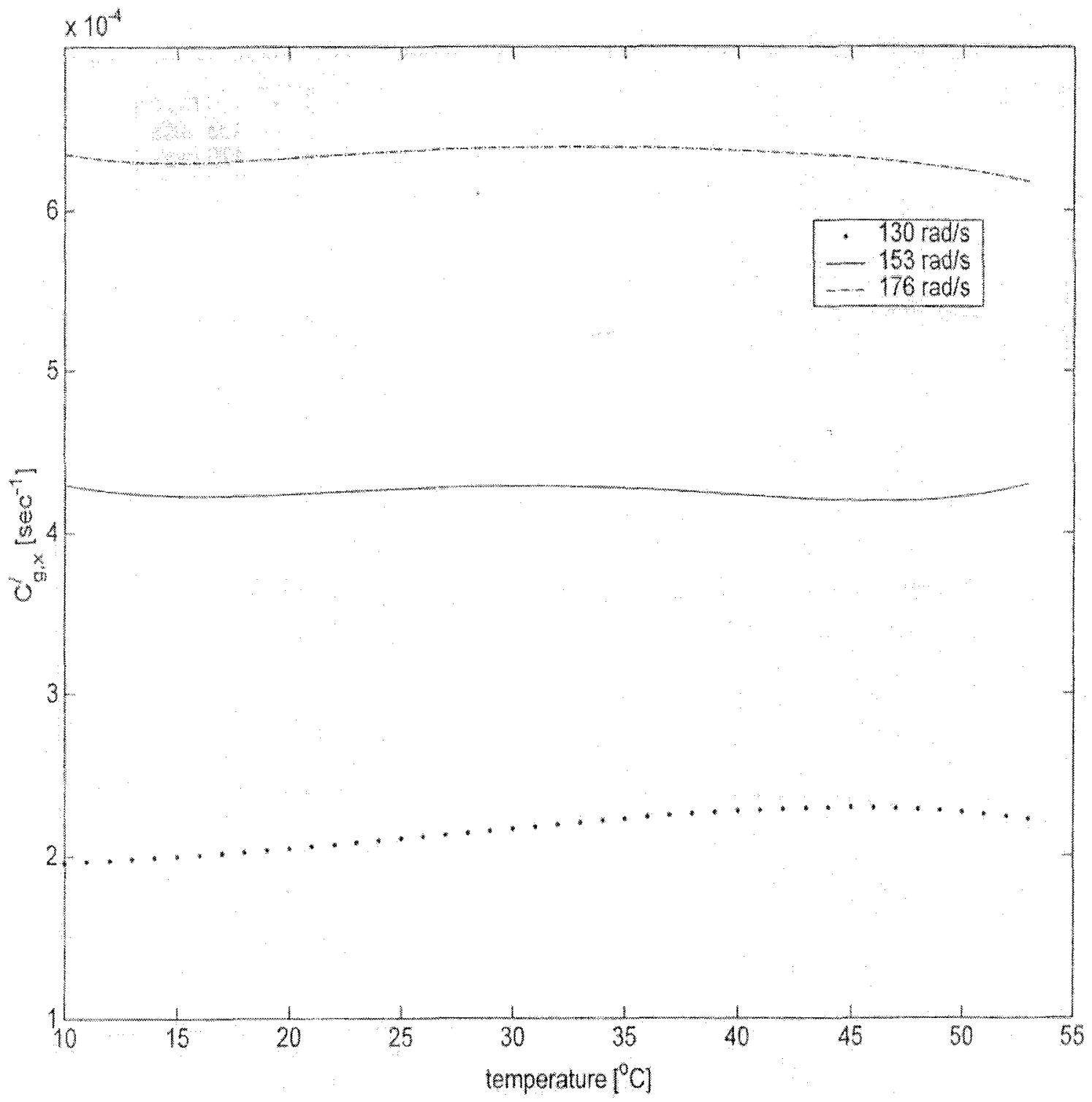

Figure C.5: Plot of calibrated value for $c_{g, x}^{\prime}$ versus $T_{h, y}$ for $\phi_{x}=0^{\circ}$ and $\phi_{y}=0^{\circ}$; $\omega_{z}=130 \mathrm{rad} / \mathrm{s}, 153 \mathrm{rad} / \mathrm{s}$, and $176 \mathrm{rad} / \mathrm{s}$. 


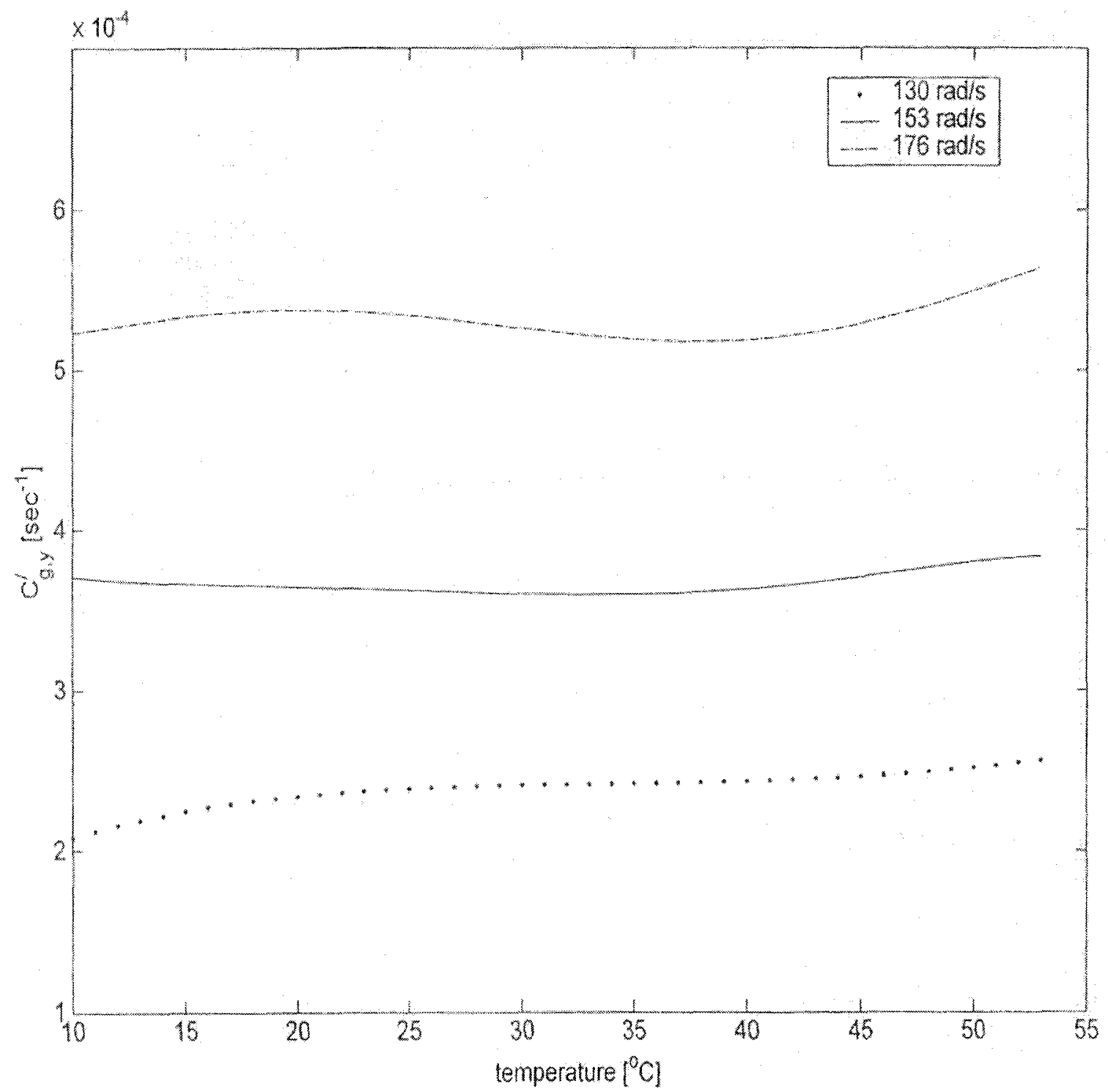

Figure C.6: Plot of calibrated value for $c_{g, y}^{\prime}$ versus $T_{h, x}$ for $\phi_{x}=0^{\circ}$ and $\phi_{y}=0^{o}$; $\omega_{z}=130 \mathrm{rad} / \mathrm{s}, 153 \mathrm{rad} / \mathrm{s}$, and $176 \mathrm{rad} / \mathrm{s}$. 


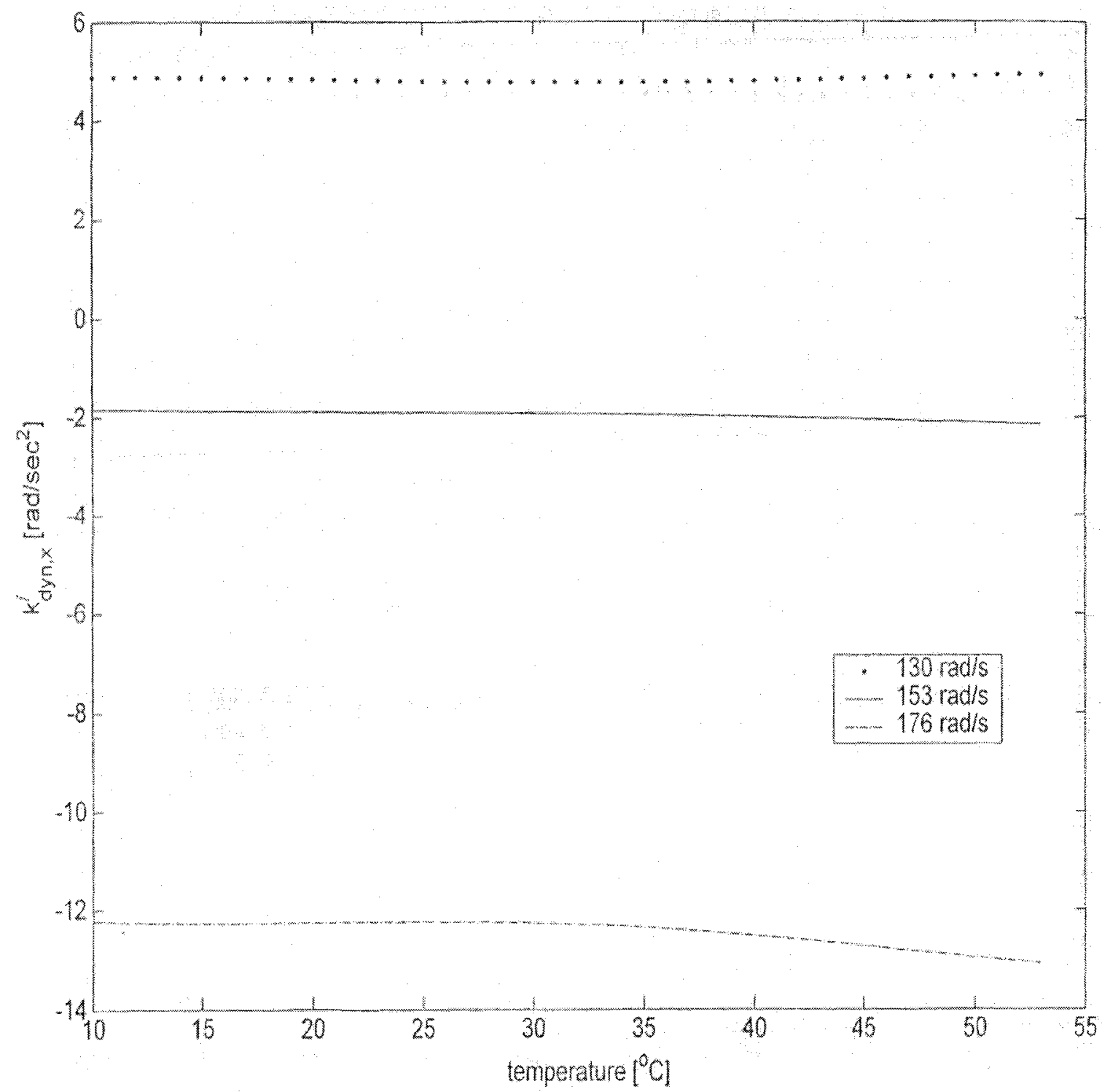

Figure C.7: Plot of calibrated value for $K_{\mathrm{dyn}, y}^{\prime}$ versus $T_{h, y}$ for $\phi_{x}=0^{\circ}$ and $\phi_{y}=0^{\circ}$; $\omega_{z}=130 \mathrm{rad} / \mathrm{s}, 153 \mathrm{rad} / \mathrm{s}$, and $176 \mathrm{rad} / \mathrm{s}$. 


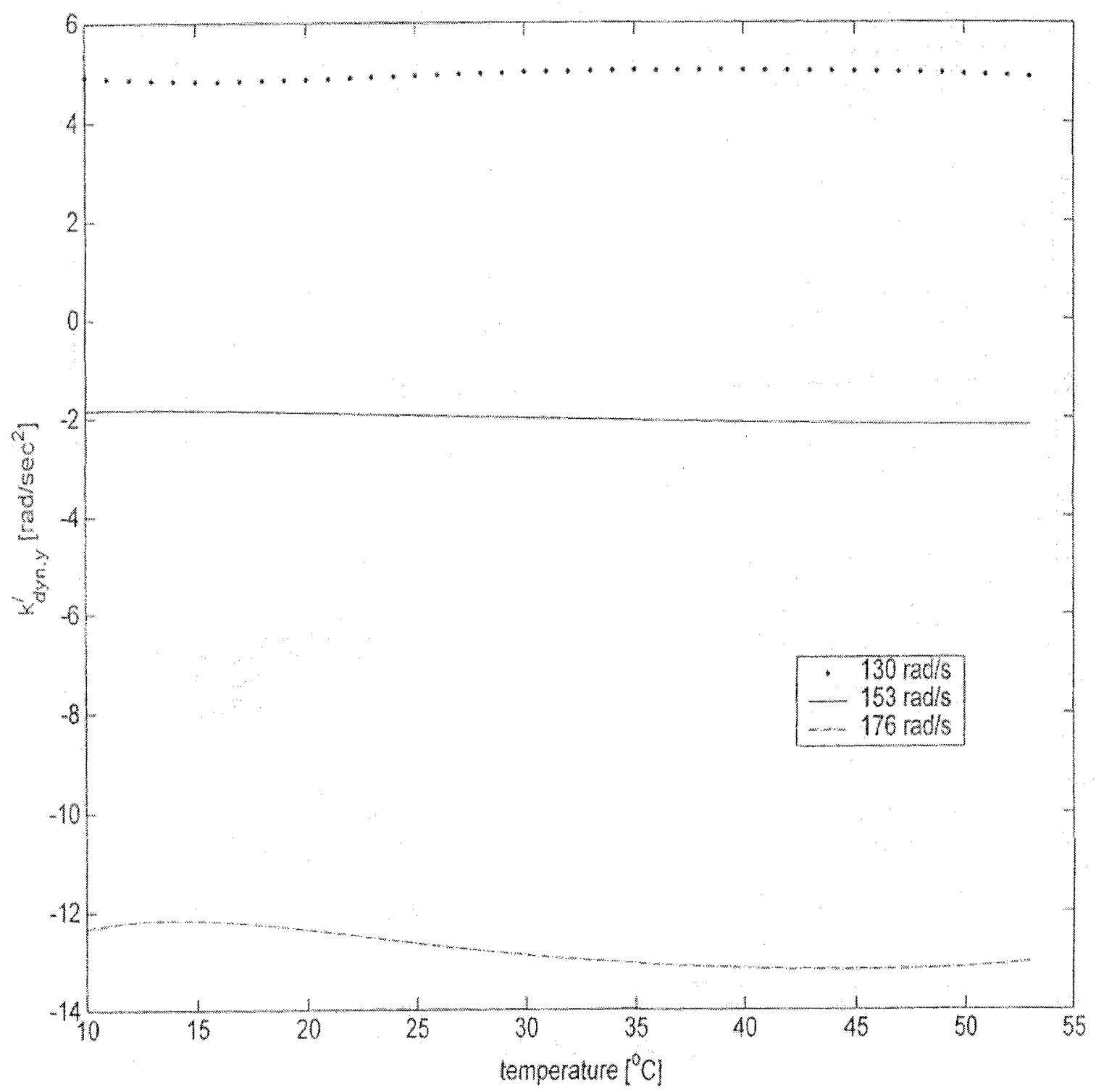

Figure C.8: Plot of calibrated value for $K_{\mathrm{dyn}, y}^{\prime}$ versus $T_{h, x}$ for $\phi_{x}=0^{\circ}$ and $\phi_{y}=0^{\circ}$; $\omega_{z}=130 \mathrm{rad} / \mathrm{s}, 153 \mathrm{rad} / \mathrm{s}$, and $176 \mathrm{rad} / \mathrm{s}$. 